\title{
Utilization of the Differential Die-Away Self-Interrogation \\ Technique for Characterization and Verification \\ of Spent Nuclear Fuel
}

by

\author{
Alexis Chanel Trahan \\ A dissertation submitted in partial fulfillment \\ of the requirements for the degree of \\ Doctor of Philosophy \\ (Nuclear Engineering and Radiological Sciences) \\ in the University of Michigan \\ 2016
}

Doctoral Committee:

Professor Sara A. Pozzi, Chair

Assistant Professor Christine Aidala

Assistant Professor Marek Flaska, Pennsylvania State University

Vladimir Henzl, Los Alamos National Laboratory

Professor William R. Martin 
(C) Alexis Trahan

2016 
For Travis, the love of my life. Without his encouragement, laughter, and support, this would not have been possible.

And for my family, the single greatest source of happiness and strength in my life. 


\section{ACKNOWLEDGEMENTS}

I was fortunate to receive a great deal of support, academically and personally, throughout the duration of my thesis work. My research advisor Professor Sara Pozzi challenged me and always pushed me to achieve more, which has served me greatly. She has also been flexible and supportive even when my career has not followed the typical path. My many mentors at Los Alamos including Anthony, Vlad, Martyn, Howard, Johnna and Stacey have given me their time, patience, expertise, and endless support in my years studying with them, and their guidance was an essential part of my graduate school experience. Alison, Karen, Margie, Adrienne and Katrina helped me work through several challenging problems in Los Alamos and I cannot thank them enough. Andreas Enqvist and Marek Flaska provided me innumerable hours of feedback on my publications and research in general. Their guidance was invaluable.

My husband and my entire family have been my greatest support system throughout graduate school and my entire life. Travis has supported me both emotionally and technically, spending many nights listening to me work physics problems out loud and helping me through them. Ryan and Mom have soothed me through the most difficult days and nights of college, always happy to answer my panicked phone calls and visit me wherever in the world I'm living. Dad and Wendy celebrated every small achievement and milestone with me and never let me forget how proud they were. Natasha has been supportive and helpful all throughout my college years and Auntie and Uncle have always been there for me, no matter what was going on in my life. Baba is the strongest person I know and has loved and cheered for me unconditionally. She has never let me believe in limits, and she taught me that there are always more places to go and people to see.

I want to thank all of my friends in Michigan, many of whom studied for classes and quals with me, and all of whom made my years in Ann Arbor some of my best. Katie and Alison were there through the toughest parts of my graduate studies, always free for a pick-me-up and a night off when I needed it most. Cayla has been the most wonderful, loyal friend since we met almost 15 years ago. I 
am eternally grateful to all who helped make this a reality and who provided support throughout this challenging and rewarding process.

This work was supported by the U.S. Department of Homeland Security under Grant Award Number 2012-DN-130-NF0001-02. The views and conclusions contained in this document are those of the authors and should not be interpreted as necessarily representing the official policies, either expressed or implied, of the U.S. Department of Homeland Security or the NNSA.

The work was also supported by the Next Generation Safeguards Initiative (NGSI), Office of Nonproliferation and Arms Control (NPAC), National Nuclear Security Administration (NNSA). 


\section{TABLE OF CONTENTS}

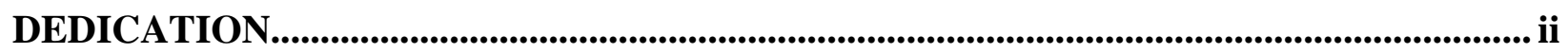

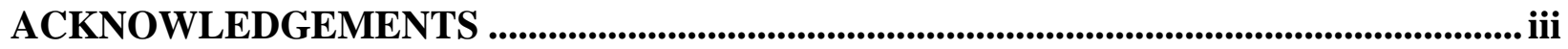

LIST OF FIGURES ....................................................................................................... vii

LIST OF TABLES ................................................................................................................ xvi

LIST OF APPENDICES .............................................................................................................

LIST OF ABBREVIATIONS ......................................................................................

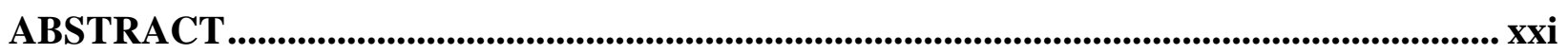

CHAPTER 1. INTRODUCTION AND THEORY _...................................................... 1

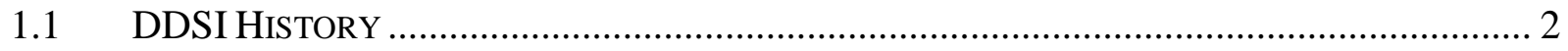

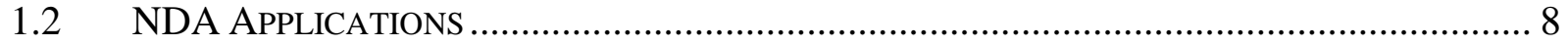

1.3 COMPARISON OF DDSI METHOD WITH OTHER NDA TECHNIQUES …............................. 9

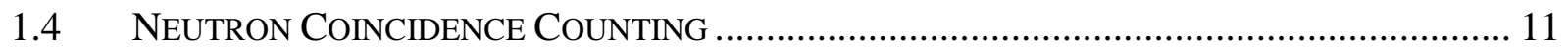

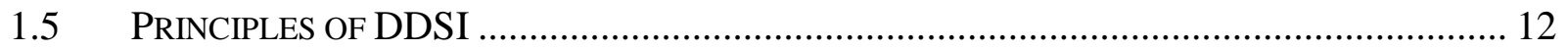

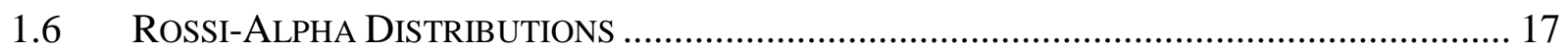

CHAPTER 2. INSTRUMENT HARDWARE AND DATA ACQUISITION..................... 20

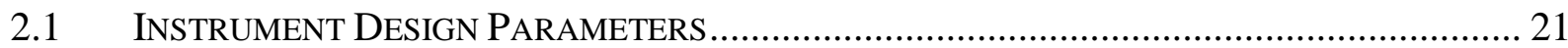

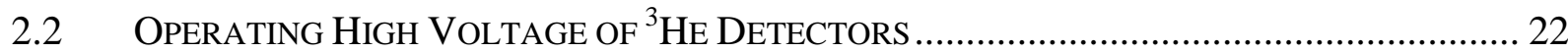

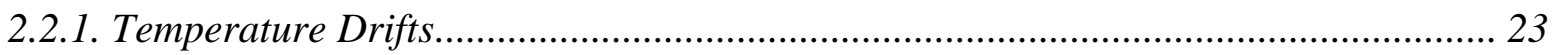

2.2.2. Gamma Dose

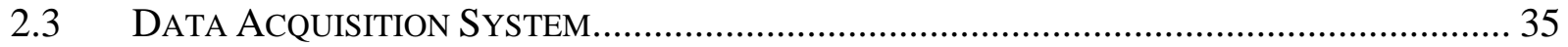

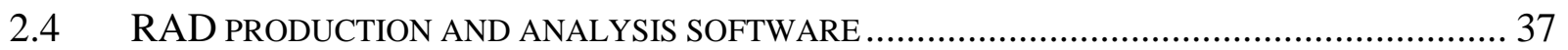

CHAPTER 3. SPENT NUCLEAR FUEL .................................................................... 41

3.1 ISOTOPIC CONTENT AND CHANGES WITH REACTOR VARIABLES ..................................... 42

3.2 SiMULATING SPENT FUEL WITH MCNP ……………............................................. 44

3.2.1. Simulating the Spontaneous Fission Source in Spent Fuel........................................ 45

3.3 Neutron Production Methods in SPent Fuel and Relative Prominences ........ 48

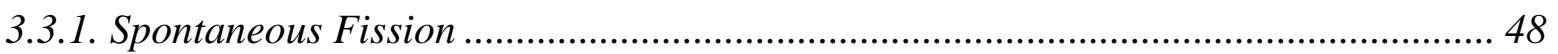

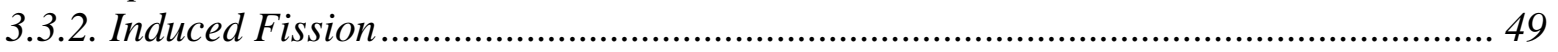

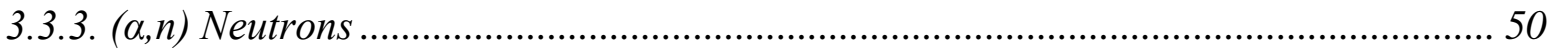

3.3.4. Photo-Fission Neutrons ............................................................................... 51

3.4 ENGineERING ANd Physical DifFerences Between Fresh AND SPENT Fuel .......... 52

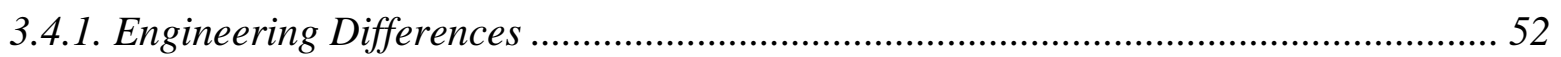

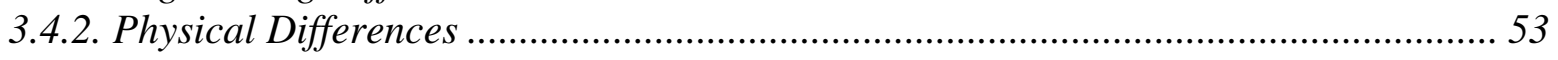

CHAPTER 4. SIMULATING THE DDSI INSTRUMENT RESPONSE ............................. 55 


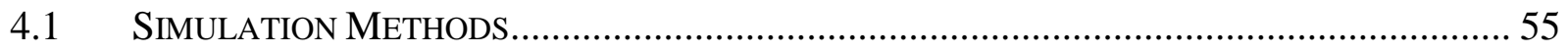

4.1.1. RADs from Simulated Pulse-Trains with MCNPX-PoliMi ....................................... 55

4.1.2. Reals-Only RADs from F8 Capture Tallies in MCNP ............................................ 58

4.1.3. Comparison of Pulse Train and F8 Methods......................................................... 59

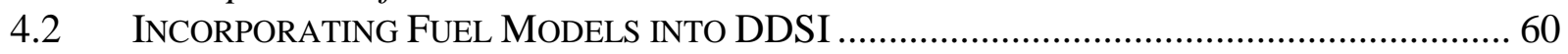

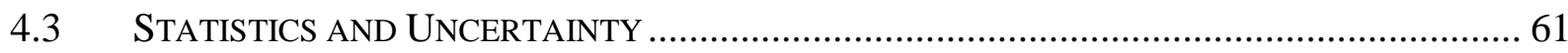

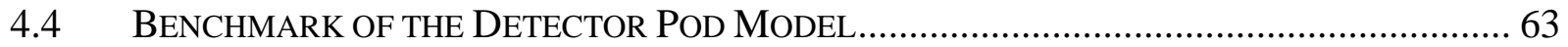

CHAPTER 5. ANALYSIS OF SIMULATED SPENT FUEL ASSEMBLY ASSAY ........ 66

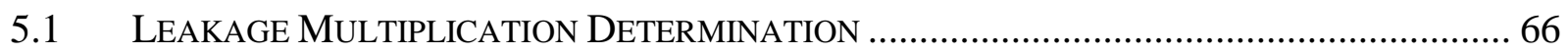

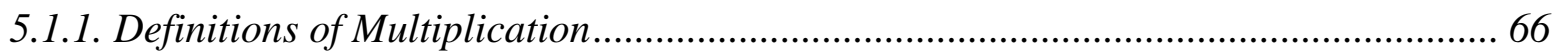

5.1.2. Traditional Leakage Multiplication Determination Methods ....................................... 69

5.1.3. Utilization of the RAD and Early Die-Away Time Concept......................................... 70

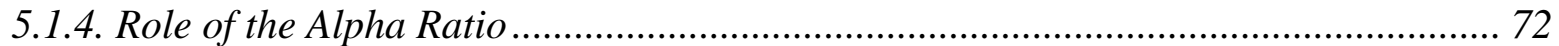

5.1.5. Results for Different Spent Fuel Libraries................................................................. 73

5.2 SPENT NuCLEAR Fuel ASSEMBly Plutonium MasS CALCULATION.............................. 78

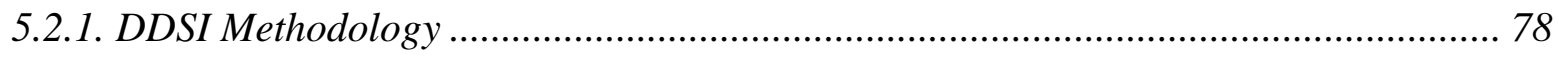

5.2.2. Results from Various Spent Fuel Libraries............................................................. 80

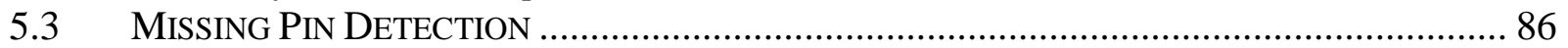

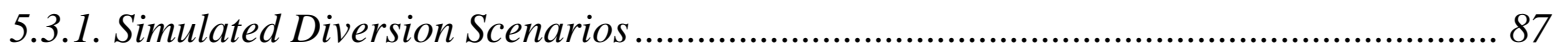

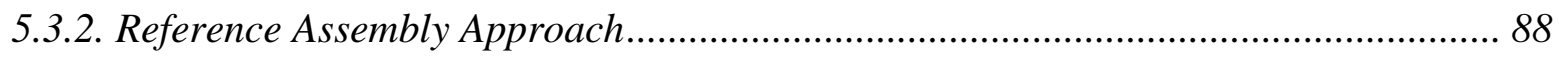

5.3.3. Changes in Total Plutonium Mass Approach ………............................................ 93

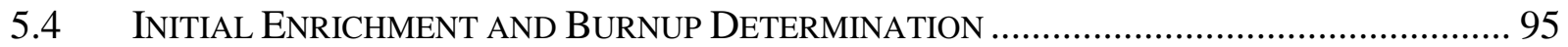

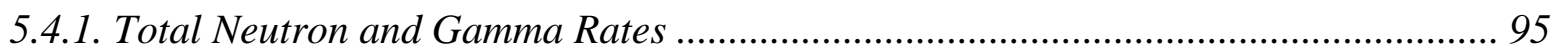

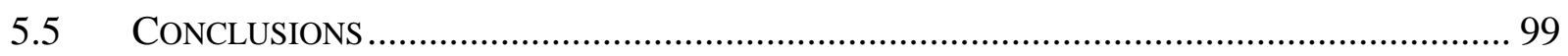

CHAPTER 6. INSTRUMENT CHARACTERIZATION EXPERIMENTS ..................... 102

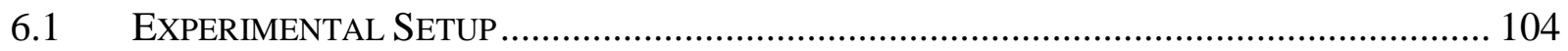

6.2 COMPARISON OF SHIFT REGISTER AND LIST MODE RESULTS ...................................... 105

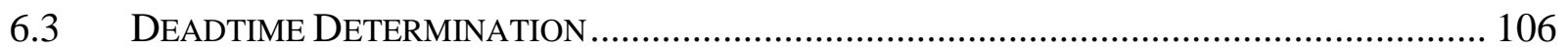

6.4 DiE-AWAY TIME AND EFFICIENCY DETERMINATION …………….............................. 107

6.5 BENCHMARK OF CHARACTERIZATION MEASUREMENTS ……..................................... 108

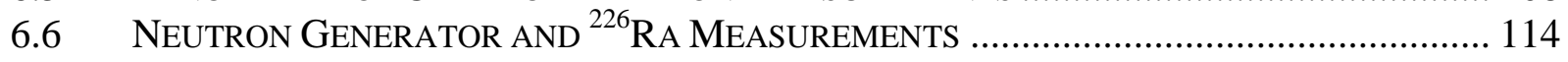

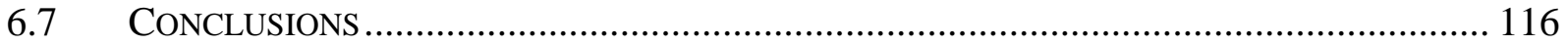

CHAPTER 7. FRESH NUCLEAR FUEL EXPERIMENTS ................................................ 117

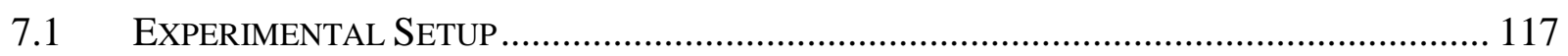

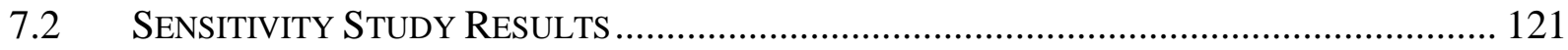

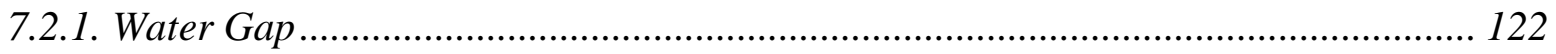

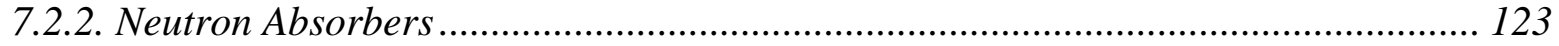

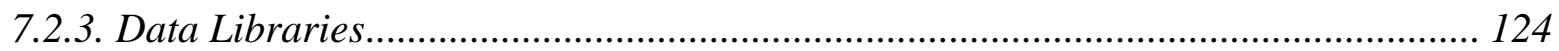

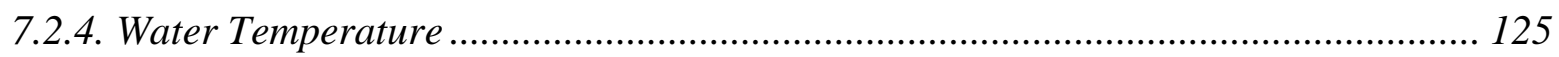

7.2.5. Conclusions of the Sensitivity Study ……….................................................. 125

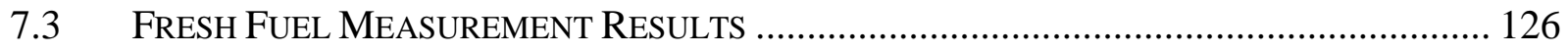

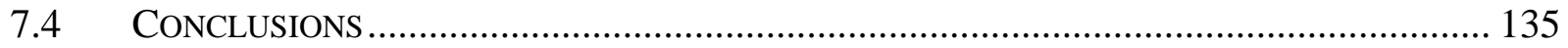


CHAPTER 8. ROSSI-ALPHA DISTRIBUTIONS WITH ORGANIC LIQUID

SCINTILLATORS............................................................................................................................. 136

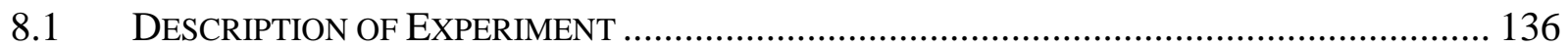

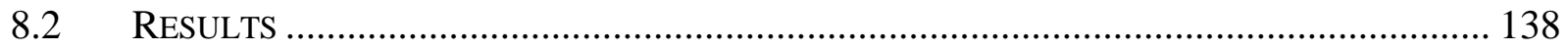

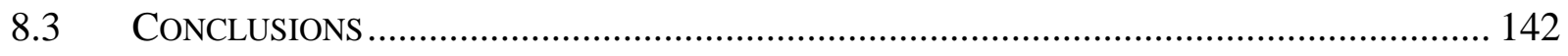

CHAPTER 9. CONCLUSIONS …............................................................................................. 143

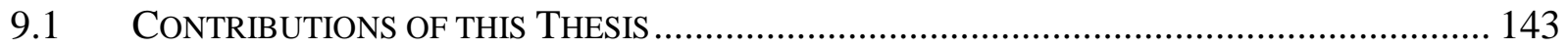

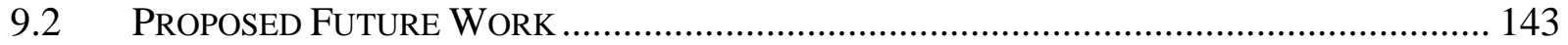

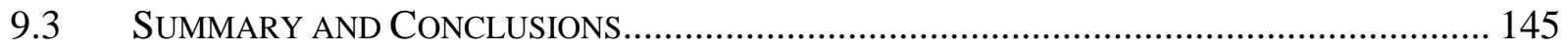

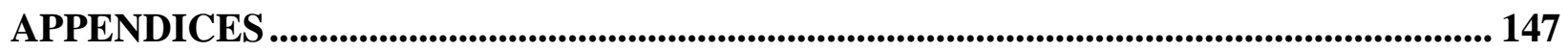

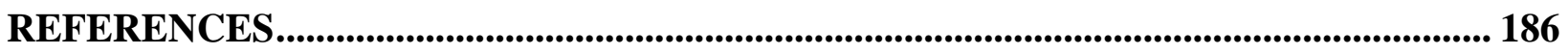




\section{LiST OF FiguRES}

Figure 1-1. Cross-section of the original DDSI cylindrical design. Detector efficiency is $13 \%$ and die-away time is $22 \mu \mathrm{s}$.

Figure 1-2. Conceptual time correlation distribution indicating data from different regions and early

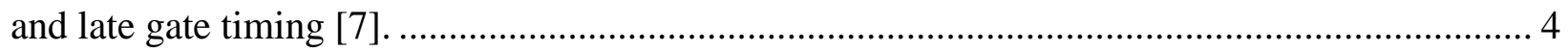

Figure 1-3. Cross-section of the three-sided DDSI design. Detector efficiency is $10 \%$ and die-away

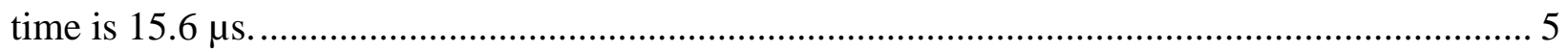

Figure 1-4. Cross-section of the final, four-sided DDSI design. Detector efficiency is $11.7 \%$ and die-

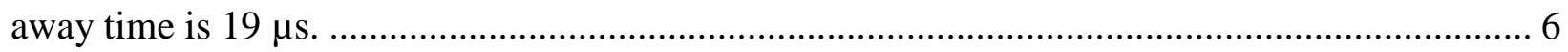

Figure 1-5. Final DDSI design with component measurements listed in Table 1-I. ................... 7 Figure 1-6. Neutron induced fission cross-sections of common fissile and fertile isotopes in SNF, from ENDF-VII. The dashed line indicates thermal neutron energy. [37] ............................... 13

Figure 1-7. Example of a simulated RAD from assay of a SFA with $15 \mathrm{GWd} / \mathrm{tU}$ BU, $3 \%$ IE, and 20 year CT. The RAD is decomposed into additive single exponentials: the fast and slow components.

Figure 1-8. Example of a simulated RAD from assay of a SFA with $15 \mathrm{GWd} / \mathrm{tU}$ BU, 3\% IE, and 20 year CT with early die-away curve shown in green squares. ............................................. 17 Figure 1-9. Visual representation of a pulse train, horizontal axis represents time; tick marks represent neutron detection event. Time window considered for RAD construction is shown in red. 17

Figure 1-10. Changing simulated RAD shape as a function of fissile/fertile ratio..................... 18 Figure 1-11. Changing RAD shape as a function of burnup in simulated spent fuel assemblies. 19 Figure 2-1. Images of the DDSI instrument. Instrument side view (top left), instrument top view with fuel rack and detector enclosures sealed (top right), open detector enclosure (bottom left), top view with three empty detector enclosures awaiting detector pods (bottom right). 
Figure 2-2. Effect of Cd on die-away time of a generic, simulated spent fuel assembly. "Original" has $\mathrm{Cd}$ surrounding all sides of the detector pods; "No $C d$ " has bare pods. The difference in die-away time is approximately $30 \mu \mathrm{s}$.

Figure 2-3. Funnels created for assembly placement in DDSI. Designed according to specifications set forth by the Swedish interim storage facility where testing of DDSI is to occur. 22 Figure 2-4. Experimental setup in HLNC well counter. S1, S2, and S3 indicate corresponding setups as discussed above. $\mathrm{H} 1, \mathrm{H} 2, \mathrm{H} 3$ and $\mathrm{H} 4$ are the empty slots in which additional temperature measurements were taken. 24 Figure 2-5. High voltage plateaus for three setups. ............................................................ 24

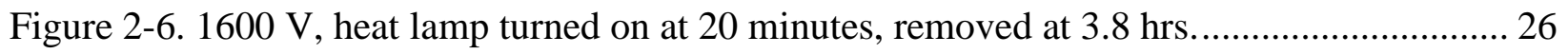

Figure 2-7. $1620 \mathrm{~V}$, heat lamp turned on at 0 minutes (start), removed after 3 hrs.................. 26 Figure 2-8. $1640 \mathrm{~V}$, heat lamp turned on at 20 minutes, removed after 6.8 hrs. ...................... 27

Figure 2-9. $1660 \mathrm{~V}$, heat lamp turned on at 20 minutes, removed after $5.5 \mathrm{hrs}$...................... 27

Figure 2-10. $1680 \mathrm{~V}$, heat lamp turned on at 20 minutes, removed after 4.7 hrs. ................... 27

Figure 2-11. $1700 \mathrm{~V}$, heat lamp turned on at 20 minutes, removed after 4 hrs. ....................... 28

Figure 2-12. Experimental setup schematic. ${ }^{252} \mathrm{Cf}$ source was affixed to the back of the pod between the two detectors in use, marked in red. Detector 1 was paired with the PDT-110A amplifier and detector 3 was paired with the A111 amplifier. The radium source was placed in increments of 10

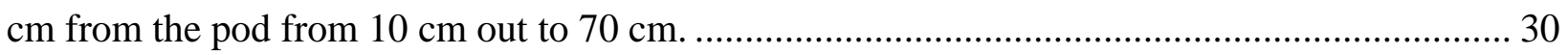

Figure 2-13. Picture taken of radium source at $20 \mathrm{~cm}$ distance.......................................... 31 Figure 2-14. High voltage plateaus with ${ }^{252} \mathrm{Cf}$ source alone for A111 and PDT-110A preamplifiers.

Figure 2-15. High voltage plateaus measured with A111 preamplifier with ${ }^{226}$ Ra source at varying distances. For the design basis of $20 \mathrm{Rad} / \mathrm{hr}$ at $20 \mathrm{~cm}$, the deviation voltage is $1640 \mathrm{~V}$. 33 Figure 2-16. High voltage plateaus measured with PDT-110A preamplifier with ${ }^{226} \mathrm{Ra}$ source at varying distances. For the design basis of $20 \mathrm{Rad} / \mathrm{hr}$ at $20 \mathrm{~cm}$, the deviation voltage is $1620 \mathrm{~V} .33$ Figure 2-17. Count rate demonstrating neutron detection efficiency at different gamma doses. Efficiency remains constant within statistical uncertainties as the dose increases. $1 \sigma$ uncertainties are shown. 35

Figure 2-18. Simulated RAD from ${ }^{252} \mathrm{Cf}$ spontaneous fission source in DDSI, which exhibits only a single exponential component. 38 
Figure 2-19. RAD with slow component shown. Fit is from 100-200 $\mu$ s and extrapolated back to 0 $\mu \mathrm{s}$.

Figure 2-20. RAD (in green) with slow component subtracted leaving another single exponential from which the fast component will come. 39

Figure 2-21. RAD with slow and fast fits shown. 40

Figure 3-1. Cross-sectional schematic of a PWR fuel assembly used in MCNP simulations with 25 empty channels for control rods and 264 fuel pins. 41

Figure 3-2. Production schematic for high-z transuranics through the fuel burning process. Neutron absorption processes are depicted by horizontal arrows. 43

Figure 3-3. Mass of ${ }^{240} \mathrm{Pu}$ and ${ }^{244} \mathrm{Cm}$ in simulated $5 \mathrm{y}$ cooled fuel assemblies as a function of BU and IE. Mass increases with decreasing IE and increases with increasing BU. 44 Figure 3-4. Emitted neutron energy distribution from ${ }^{244} \mathrm{Cm}$ spontaneous fission source in SFL2a simulated $45 \mathrm{GWd} / \mathrm{tU}$ BU, $4 \%$ IE, 5 y CT assembly.

Figure 3-5. Simulated emitted neutron energy distribution from a typical $(\alpha, n)$ source in spent fuel.

Figure 4-1. RAD produced with pulse-train data simulated with MCNPX-PoliMi.................. 57

Figure 4-2. Reals-only RAD produced with F8 tally. The die-away time is $19.4 \mu$ s................ 59 Figure 4-3. Reals-only RAD produced with PTRAC output compared to RAD from F8 tally. The agreement is exact indicating that the F8 RAD is identical to the PTRAC output when accidentals are omitted. 60

Figure 4-4. Singles rate in detectors as a function of axial distance of source emissions from center of the $45 \mathrm{GWd} / \mathrm{tU} \mathrm{BU}, 4 \% \mathrm{IE}$, and 5 y CT assembly. 61 Figure 4-5. Signal/Background (S/B) ratio for slow and fast components and early die-away region. The S/B ratio is consistently favorable for the early die-away region because of the higher magnitude of the signal. 62 Figure 4-6. Time where reals fall lower than $1 \sigma$ Accidentals for four assemblies of interest. These represent the limits of where the accidentals gate can be measured. To accommodate the low count rate $5 / 15 / 5$ case, at least $800 \mu$ s would need to precede the accidentals gate.

Figure 4-7. Detector configuration for benchmarking measurements. Detectors outlined in red were used in the experiment. 
Figure 4-8. Experimental RAD with accidentals subtracted (top left), simulated reals-only RAD (top right), and comparison (bottom). Agreement is very good both in magnitude and doubles rate. 64 Figure 5-1. Application of $(\mathrm{L} / \mathrm{E})_{\mathrm{D}}$ measurement to Fukushima fuel debris, from [71] .............. 70 Figure 5-2. Comparison of four SFAs with various values of IE, BU, and CT. The relative magnitudes of the fast and slow components shape the die-away time of the total RAD in the early time domain. The statistical uncertainties on the RAD values are smaller than the markers used.71 Figure 5-3. Exponential fit of the early time domain of an RAD shown with squares. The early-die away time is determined from this function. In this example, it is $40 \mu \mathrm{s}$. The uncertainties on the RAD values are smaller than the markers used. 72 Figure 5-4. Net multiplication as a function of early die-away time calculated by fitting an exponential to the early time domain of a simulated RAD. A linear fit is applied with a slope of 0.05 $\mu$ s. The $1 \sigma$ uncertainties on the early die-away time are given. Uncertainties on the net multiplication values are smaller than the markers used. 74

Figure 5-5. Relative difference in predicted and computed net multiplication for 44 SFAs from SFL2a. Linear fit (top) and 2nd order polynomial fit (bottom). Mean variation is $1.4 \%$ for the linear fit and $0.7 \%$ for the $2^{\text {nd }}$ order polynomial. The legend is the same that was used for Figure 5-4. From the published work [9]. 74 Figure 5-6. Multiplication vs. early die-away time, as a function of operating parameter. Fit from SFL2a is shown with a solid line. The $1 \sigma$ uncertainties on the early die-away time are given. Uncertainties on the net multiplication values are smaller than the markers used..................... 76 Figure 5-7. Multiplication vs. early die-away time as a function of burnup. Fit from SFL2a is shown with a solid line. The $1 \sigma$ uncertainties on the early die-away time are given. Uncertainties on the net multiplication values are smaller than the markers used. .................................................... 76 Figure 5-8. Multiplication vs. early die-away time for SFL4 assemblies. The $1 \sigma$ uncertainties on the early die-away time are given. Uncertainties on the net multiplication values are smaller than the

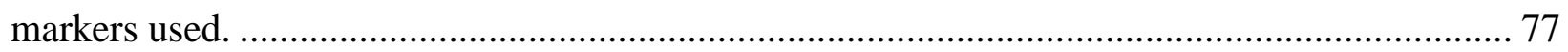

Figure 5-9. Plots for each cooling time to determine calibration parameters.......................... 80 Figure 5-10. Results of the simulations of the detected singles rate $\mathrm{S}($ left $)$ and $\tau_{\mathrm{e}}($ right $)$ for 44 SFAs from SFL2a as measured by the DDSI instrument. 82 
Figure 5-11. Comparison of the absolute values of the $m_{P u}$ determined from eq. (6) and the true $m_{P u}$ in the SFA (left) and the relative differences between the determined $\mathrm{m}_{\mathrm{Pu}}$ and the true values (right).

Figure 5-12. Values of the fitting parameters $d$ and $f$ from eq. (6) and $a$ and $b$ from eq.(16) from [33], as a function of CT. 84

Figure 5-13. Pu determination algorithm applied to SFL3 data to determine calibration constants. Different constants are needed for different burnups, as demonstrated here. 85

Figure 5-14. Difference in predicted and actual $\mathrm{Pu}$ mass as a function of $\mathrm{Pu}$ mass in SFL4 assemblies.

Figure 5-15. Diversion scenarios: center (left) and dispersed (right). Both scenarios have 40 pins (red) that have been replaced by pins with either SS or NU. 88

Figure 5-16. Standard $45 \mathrm{GWd} / \mathrm{tU}, 4 \%$, 5 year cooled assembly with all pins intact. Early die-away time $=40 \mu \mathrm{s}$, fast die-away time $=19.0 \mu \mathrm{s}$, and the slow die-away time $=80 \mu \mathrm{s}$. 89 Figure 5-17. 45, 4, 5 assembly with 40 pins replaced with NU. Center diversion (left) and dispersed diversion (right). 89

Figure 5-18. 45, 4, 5 assembly with 40 pins replaced with stainless steel. Center diversion (left) and dispersed diversion (right). . 90 Figure 5-19. Percent difference between fast/slow magnitude for complete and diverted assemblies as a function of diverted assembly fast magnitude. $3 \sigma$ error bars are shown. 92 Figure 5-20. Multiplication vs. early die-away time for assemblies with diversion schemes. Solid curve indicates quadratic fit obtained from complete SFL2a assemblies. 93 Figure 5-21. Relationship between total gamma, burnup, and cooling time. Power fits for each enrichment overlap, indicating that a single fit is appropriate...... 96 Figure 5-22. Relationship between burnup, initial enrichment, and neutron singles rate for each cooling time for spent nuclear fuel assemblies in SFL2a. 96

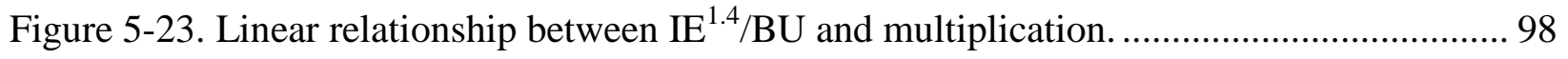
Figure 5-24. 2nd order polynomial relationship between BU/IE and Fast/Total doubles........... 98 Figure 6-1. Picture of DDSI instrument with detector pods open. Detector pods are sealed with lead and stainless steel covers before measurements take place. 103 Figure 6-2. Sealed DDSI instrument in measurement tank. 103 
Figure 6-3. High Voltage plateaus for detector/preamp sets 1, 10, 20, 30, and 40. Agreement is within $1.5 \%$ between the different sets. 104

Figure 6-4. RAD from experimental list mode data with $2 \mu$ s bins. Die-away time from fit to RAD is $18.1 \mu \mathrm{s}$. 108

Figure 6-5. 3D VisEd DDSI simulation (left), xy plane cross sectional view of DDSI simulation (center), and yz cross sectional view of DDSI simulation (right). 109

Figure 6-6. Simulated and experimental RADs from a single ${ }^{252} \mathrm{Cf}$ source in the DDSI instrument. 110

Figure 6-7. Comparison of channel-by-channel singles for experiment and simulation. 110

Figure 6-8. Channel-by-channel comparison between experiment and simulation when source is centered in simulation, but likely off-center in experiment.

Figure 6-9. Channel-by-channel singles comparison for ${ }^{252} \mathrm{C}$ source slightly off-center in water with no fuel rack. 112

Figure 6-10. Overlain RADs for ${ }^{252} \mathrm{Cf}$ source centered in water with no fuel rack. 112 Figure 6-11. Channel-by-channel singles comparison from the measurement of a ${ }^{252} \mathrm{Cf}$ source in the center guide tube of a fuel rack, with the entire instrument submerged in water. 113

Figure 6-12. Comparison of experimental and simulated RADs for ${ }^{252} \mathrm{Cf}$ source center guide tube in water with fuel rack. 113

Figure 6-13. Neutron generator centered among DDSI detector pods. 115

Figure 7-1. Fuel rack centered in DDSI instrument. 118

Figure 7-2. Simulated neutron flux from axially and radially distributed ${ }^{252} \mathrm{Cf}$ sources in the fresh fuel assembly. 119

Figure 7-3. Thirteen fuel rod configurations with varying effective enrichments created with LEU, $\mathrm{DU}$, and $\mathrm{LEU}+$ gadolinium rods. 120

Figure 7-4. Three different fuel rod configurations with the same effective enrichment but varying degrees of asymmetric distribution of LEU and DU rods. (a) 1.67\% fully asymmetric loading, (b) $1.67 \%$ partially asymmetric loading (b), and (c) reversed $1.67 \%$ partially asymmetric loading.121 Figure 7-5. Channel-by-channel comparison of experimental and simulated data for base case of five ${ }^{252} \mathrm{Cf}$ sources in the otherwise empty fuel rack in water..................................................... 122 Figure 7-6. Ratio of May/October singles and doubles rates............................................. 126 
Figure 7-7. Comparison of die-away times of experimental and simulated RADs from $0.21 \%$ enrichment case.

Figure 7-8. Overlain RADs from $0.21 \%$ enrichment case.

Figure 7-9. Comparison of die-away times of experimental and simulated RADs from $0.98 \%$ enrichment case. 130

Figure 7-10. Overlain RADs from $0.98 \%$ enrichment case. 130

Figure 7-11. Comparison of die-away times of experimental and simulated RADs from $1.97 \%$ enrichment case.

Figure 7-12. Overlain RADs from $1.97 \%$ enrichment case.

Figure 7-13. Comparison of die-away times of experimental and simulated RADs from 1.67\%, fully asymmetric case. 132

Figure 7-14. Overlain RADs from $1.67 \%$, fully asymmetric case. 132

Figure 7-15. Net multiplication as function of singles rate for measured fresh fuel data (left), and simulated spent fuel data (right).

Figure 7-16. Measured singles rate as a function of total ${ }^{235} \mathrm{U}$ content in all fuel rods. Polynomial is extended back to 0 to determine zero-fissile mass singles rate. 134

Figure 7-17. Leakage multiplication, which is measured single rate over intercept singles rate, as a function of early die-away time for measured fresh fuel data. Asymmetric assemblies are denoted with square markers. 134

Figure 8-1. BeRP ball measurement setup. Image from [97]. 137

Figure 8-2. RAD from bare BeRP ball setup with exponential fit shown. 138

Figure-8-3. Normalized RADs for BeRP measurement setups. 139

Figure 8-4. RAD from measurement with 0.5 " W reflector, with fast and slow curves shown. 141 Figure 8-5. RAD from measurement with 1"W reflector, with fast and slow curves shown.... 141 Figure 8-6. RAD from measurement with 1" HDPE reflector, with fast, slow, and early curves shown. 141

Figure 8-7. RAD from measurement with 3" HDPE reflector, with fast, slow, and early curves shown. 141

Figure A-1. Visual representation of the source distances from the detector face. ${ }^{252} \mathrm{Cf}$ is stationary at a distance of $10 \mathrm{~cm}$ and ${ }^{137} \mathrm{Cs}$ is placed at varying distances from the detector, indicated by the tick marks .148 
Figure A-2. Experimental setup for the ${ }^{137} \mathrm{Cs}$ source placed at $5 \mathrm{~cm}$ from the detector face and ${ }^{252} \mathrm{Cf}$ source at $10 \mathrm{~cm}$ from the detector face

Figure A-3. Count rates as a function of ${ }^{137} \mathrm{Cs}$ distance from detector face, without background subtraction. The count rate of the ${ }^{137} \mathrm{Cs}$ source at the distance of $175 \mathrm{~cm}$ is just above the background

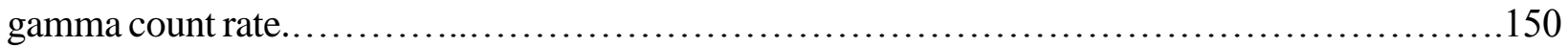

Figure A-4. Examples of clipped and double pulses.

Figure A-5. Example of taking a light output "slice" from the 2-D plot, and slicing again perpendicular. 152

Figure A-6. Entire PSD plot sliced with optimal separation points found. Zoomed version on the right

Figure A-7. Discrimination line applied to the ${ }^{252} \mathrm{Cf}$ alone case which is the lowest gamma-ray fluence scenario. The color scale measures the density of pulses in each region of the plot. . .

Figure A-8. Neutron PHDs for $2.5 \mathrm{~cm}$ through $10 \mathrm{~cm}{ }^{137} \mathrm{Cs}$ distances from the detector. The closestrange distances of $2.5 \mathrm{~cm}$ and $5 \mathrm{~cm}$ show a Compton edge from misclassified gammas, likely from missed double pulses.

Figure A-9. Neutron PHDs for $15 \mathrm{~cm}$ through $175 \mathrm{~cm}$ distances from the detector. These distances show close agreement with the ${ }^{252} \mathrm{Cf}$ alone case........................................... 154 Figure A-10. Percent deviation of the various ${ }^{252} \mathrm{Cf}$ and ${ }^{137} \mathrm{Cs}$ experiments compared to the ${ }^{252} \mathrm{Cf}$ alone case, for the $2.5 \mathrm{~cm}$ through $10 \mathrm{~cm}$ cases

Figure A-11. Percent deviation of the various ${ }^{252} \mathrm{Cf}$ and ${ }^{137} \mathrm{Cs}$ experiments compared to the ${ }^{252} \mathrm{Cf}$ alone case, for the $15 \mathrm{~cm}$ and $175 \mathrm{~cm}$ cases. .156 Figure A-12. Neutron count rate deviation from the ${ }^{252} \mathrm{Cf}$ alone case, with statistical uncertainties shown as standard error. The horizontal line shows where the values should be to match the ${ }^{252} \mathrm{Cf}$ alone case .157 


\section{LIST OF TABLES}

Table 1-I. Dimensions of final DDSI simulated system design........................................ 7

Table 2-I. Empty slot measurements and extrapolations to S1 and S2. Extrapolations are done using the linear adjustment found from the difference between $\mathrm{H} 3$ and $\mathrm{H} 1$, and $\mathrm{H} 4$ and $\mathrm{H} 2$............. 25 Table 2-II. Temperature measurements in the room, left and right slots, UCAS tube and preamplifier,

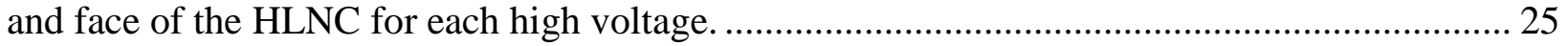
Table 2-III. Average, standard deviation, and maximum or minimum as well as deviation per degree

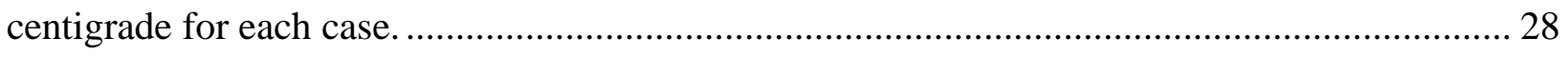

Table 2-IV. Summary of high voltage levels where deviation from ${ }^{252} \mathrm{Cf}$ plateau indicates failing

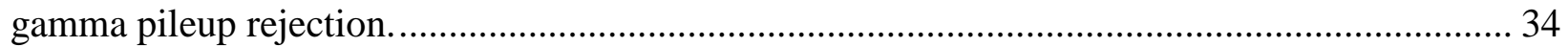
Table 2-V. Summary of TDMS file sizes and compression times for $1 \mathrm{hr}$ measurements with

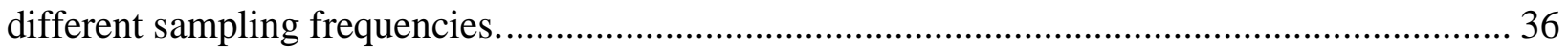
Table 3-I. Spontaneous fission isotopes (written in the format "ZZAAA" where ZZ is the atomic number and AAA is the mass number) and their contribution to the total fission rate in a $45 \mathrm{GWd} / \mathrm{tU}$

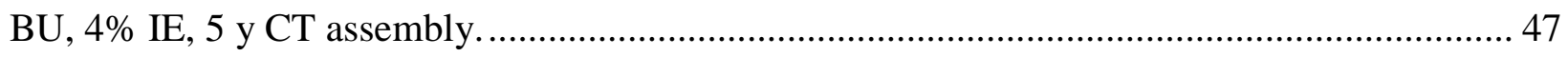

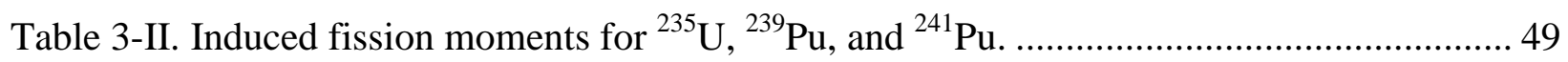
Table 3-III. Total fission contributions of fissioning isotopes (Fis) along with atom fractions (AF) for selected assemblies that demonstrate a variety in SF isotope contributions. The fission percentages to not add up to $100 \%$ because the remainder of the contributions comes from spontaneous fission. 50 Table 4-I. Summary of experiment and simulation RAD analysis results.

Table 5-I. Neutrons produced and net gain in neutrons for first several generations of a fission chain.

Table 5-II. Change in $\tau_{\text {early }}$ and D/S factors with different neutron sources. ............................ 73

Table 5-III. Description of operating parameters varied in SFL3 sensitivity study. .................. 75

Table 5-IV. Summary of true and determined $\mathrm{m}_{\mathrm{Pu}}$ for 44 SFAs from SFL2a divided into subsets by different $\mathrm{CT}$ values. 83

Table 5-V. BU/IE Combinations in SFL6. 87 
Table 5-VI. Comparison of statistics from 45GWd/tU, 4\%, 5y RADs .................................. 91

Table 5-VII. Predicted values of BU and IE with constant/known cooling time of 5y .............. 99 Table 6-I Comparison of the JSR-15 results with list mode and Rossi-alpha distribution analyzed or

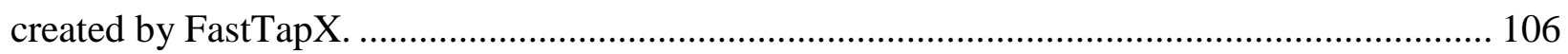
Table 7-I. Comparison of experiment with simulations in which the water gap varies from 4 to 6 $\mathrm{mm}$.

Table 7-II. Comparison of experiment with base case and added neutron absorber models for highest and lowest enrichment cases. Each absorber is added independently. 124 Table 7-III. Comparison of data library options to defaults in base case for highest and lowest

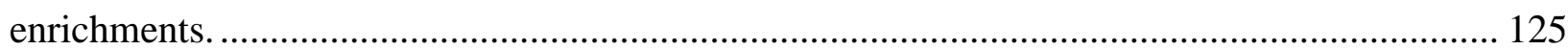

Table 7-IV. Effect of water temperature on highest and lowest enrichment cases.................. 125 Table 7-V. Comparison of the singles and doubles rates from October experiments and simulation for 13 symmetric enrichments, and 3 asymmetric loadings of $1.59 \%$ enrichment. Experimental data

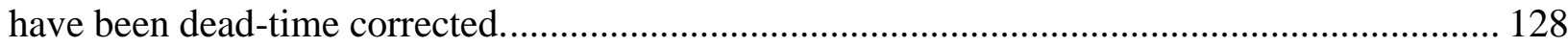
Table 8-I. Experimental parameters for different BeRP setups........................................... 137 Table A-I. Percent error as a function of distance........................................ 158 


\section{LIST OF APPENDICES}

APPENDIX A. SPENT FUEL MEASUREMENT WITH LIQUID SCINTILLATOR

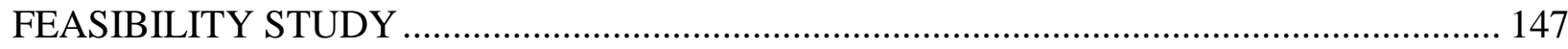

APPENDIX B. INITIAL OPERATING PARAMETERS FOR SFL4 _............................... 159

APPENDIX C. EXAMPLE MCNP INPUT FILE ………………………………............. 161

APPENDIX D. EXAMPLE PYTHON ANALYSIS SCRIPT ……………………........... 180 


\section{List of Abbreviations}

\begin{tabular}{|c|c|}
\hline$(\alpha, n)$ & Alpha-neutron reaction \\
\hline Am-Li & Americium-Lithium source \\
\hline BeRP & Beryllium Reflected Plutonium \\
\hline BU & Burnup \\
\hline BWR & Boiling Water Reactor \\
\hline $\mathrm{CH}_{2}$ & Polyethylene \\
\hline CIPN & ${ }^{252} \mathrm{Cf}$ Interrogation with Prompt Neutrons \\
\hline $\mathrm{CoK}$ & Continuity of Knowledge \\
\hline $\mathrm{CT}$ & Cooling Time \\
\hline CVD & Cherenkov Viewing Device \\
\hline DDA & Differential Die-Away \\
\hline DDSI & Differential Die-Away Self-Interrogation \\
\hline DG & Delayed Gamma \\
\hline DU & Depleted Uranium \\
\hline FDET & Fork Detector \\
\hline $\mathrm{FF}$ & Fast Fission \\
\hline GW & Gate Width \\
\hline $\mathrm{GWd} / \mathrm{tU}$ & Giga Watt Day Per Ton of Uranium \\
\hline GWe & Giga Watt Electric \\
\hline HDPE & High Density Polyethylene \\
\hline $\mathrm{HV}$ & High Voltage \\
\hline IAEA & International Atomic Energy Agency \\
\hline IE & Initial Enrichment \\
\hline LANL & Los Alamos National Laboratory \\
\hline LEU & Low-Enriched Uranium \\
\hline LSDS & Lead Slowing Down Spectroscopy \\
\hline LV & Low Voltage \\
\hline $\mathrm{NCC}$ & Neutron Coincidence Counting \\
\hline NDA & Nondestructive Assay \\
\hline NI & National Instruments \\
\hline NGSI & Next Generation Safeguards Initiative \\
\hline NU & Natural Uranium \\
\hline ORNL & Oak Ridge National Laboratory \\
\hline PD & Predelay \\
\hline PG & Passive Gamma \\
\hline PNAR & Passive Neutron Albedo Reactivity \\
\hline PWR & Pressurized-Water Reactor \\
\hline RAD & Rossi-Alpha Distribution \\
\hline RMS & Root Mean Square \\
\hline
\end{tabular}




$\begin{array}{ll}\text { SF } & \text { Spontaneous Fission } \\ \text { SFA } & \text { Spent Fuel Assembly } \\ \text { SFL } & \text { Spent Fuel Library } \\ \text { SINRD } & \text { Self-Interrogation Neutron Resonance Densitometry } \\ \text { SNF } & \text { Spent Nuclear Fuel } \\ \text { SRD } & \text { Shipper-Receiver Difference } \\ \text { SS } & \text { Stainless Steel } \\ \text { TDMS } & \text { Technical Data Management Files } \\ \text { TN } & \text { Total Neutron } \\ \text { TTL } & \text { Transistor-Transistor Logic } \\ \text { XRF } & \text { X-Ray Fluorescence }\end{array}$




\section{AbSTRACT}

New nondestructive assay techniques are sought to better characterize spent nuclear fuel. One of the NDA instruments selected for possible deployment is differential die-away self-interrogation (DDSI). The proposed DDSI approach for spent fuel assembly assay utilizes primarily the spontaneous fission and $(\alpha, \mathrm{n})$ neutrons in the assemblies as an internal interrogating radiation source. The neutrons released in spontaneous fission or $(\alpha, n)$ reactions are thermalized in the surrounding water and induce fission in fissile isotopes, thereby creating a measurable signal from isotopes of interest that would be otherwise difficult to measure. The DDSI instrument employs

neutron coincidence counting with ${ }^{3} \mathrm{He}$ tubes and list-mode-based data acquisition to allow for production of Rossi-alpha distributions (RADs) in post-processing. The list-mode approach to data collection and subsequent construction of RADs has expanded the analytical possibilities, as will be demonstrated throughout this thesis. One of the primary advantages is that the measured signal in the form of a RAD can be analyzed in its entirety including determination of die-away times in different time domains. This capability led to the development of the early die-away method, a novel leakage multiplication determination method which is tested throughout the thesis on different sources in simulation space and fresh fuel experiments. The early die-away method is a robust, accurate, improved method of determining multiplication without the need for knowledge of the $(\alpha, n)$ source term.

The DDSI technique and instrument are presented along with the many novel capabilities enabled by and discovered through RAD analysis. Among the new capabilities presented are the early die-away method, total plutonium content determination, and highly sensitive missing pin detection. Simulation of hundreds of different spent and fresh fuel assemblies were used to develop the analysis algorithms and the techniques were tested on a variety of spontaneous fission-driven fresh fuel assemblies at Los Alamos National Laboratory and the BeRP ball at the Nevada National Security Site. The development of the new, improved analysis and characterization methods with the DDSI instrument makes it a viable technique for implementation in a facility to meet material control and safeguards needs. 


\section{Chapter 1. INTRODUCTION AND THEORY}

As nuclear power expanded worldwide in the 1950s and 1960s, the production and processing of nuclear material became commonplace in a variety of states. Understanding the potential dangers of unmonitored nuclear material, the Atoms for Peace speech was delivered by President Eisenhower which led to the formation of the International Atomic Energy Agency (IAEA). In the Cold War era, the mission of preventing nuclear warfare and working toward disarmament was particularly vital. As a part of this goal, the US Nuclear Regulatory Commission, the Department of Energy, and the International Atomic Energy Agency (IAEA), among others, supported the effort to safely and accurately account for all nuclear material in order to assure its peaceful use. It was also recognized that these measurements must be rapid and nonintrusive in order to be used in a variety of facilities and states. Effective safeguards called for the reliable verification of nuclear material inventories and various nondestructive assay (NDA) techniques were developed to meet that need.

NDA, in the context of nuclear safeguards, encompasses the variety of instruments designed to characterize an item containing nuclear material without destroying or altering it [1]. The technique has a broad definition and has been used to describe a wide variety of instruments measuring almost any signal from photons and neutrons to x-rays and heat. Though typically less sensitive and accurate than destructive assay, NDA may offer several advantages including speed, repeatability, ease of operation, no sampling error (an issue with heterogeneous materials), and maintenance of sample integrity. These advantages make NDA a more attractive option for deployment, particularly when the system is passive, meaning that it does not induce radiation via an external source. The IAEA has utilized NDA techniques for nuclear facility inspections for over thirty years due to the portability and reliability these systems offer. It is desirable that the measurement technique remains under the control of the inspector throughout an inspection, making simple NDA systems particularly useful in facility inspection scenarios. Destructive analysis, alternatively, often requires that samples leave the possession of the inspector for outside analysis. 
In 2008 the Next Generation Safeguards Initiative (NGSI) was launched by the National Nuclear Security Administration to "revitalize the international safeguards technology and human resource base" [2]. Part of this effort included a five-year investigation of NDA techniques for characterization and verification of spent nuclear fuel (SNF). The primary goals were to determine total Pu mass and detect diversion of pins in commercial SNF. The project began with investigation of 14 different NDA techniques and after an initial simulation and comparison phase, six independent NDA instruments were selected for further development including integration with total neutron (TN) and passive gamma (PG) counting. One of these techniques is Differential Die-Away Self-Interrogation (DDSI) which is promising due to its passive operation and intermediate cost. The other techniques are discussed and compared in Section 1.3.

\subsection{DDSI History}

The conceptual development of the DDSI technique took place in 2008 at Los Alamos National Laboratory [3]. Howard Menlove, Spencer Menlove, and Stephen Tobin collaborated to first develop the passive, nondestructive spent fuel assay technique with the original cylindrical design shown in Figure 1-1 [4]. The design was inspired by the concept of the active differential die-away (DDA) technique which employs neutron die-away measurement following irradiation of the sample with a burst of neutrons, typically from a neutron generator [5]. The DDSI technique differed from DDA by eliminating the need for an external pulse neutron source. Instead, Menlove et al. proposed that DDSI would utilize time correlations from fission chains initiated by spontaneous fission events, which are prominent and frequent in spent fuel assemblies.

The DDSI instrument was designed to contain two distinct spatial regions to provide two different characteristic die-away times. The first was the detector region, containing ${ }^{3} \mathrm{He}$ tubes, polyethylene, and cadmium fins to decrease the detector die-away time by consuming neutrons that are already thermalized upon reaching the region. The second was the sample interrogation region, which contains the assembly in water and a layer of $\mathrm{CH}_{2}$ with optimized thickness to increase selfinterrogation without decreasing efficiency too significantly. Balance was required in the design to shorten the die-away time without losing too much efficiency. For this reason, cadmium fins were used as opposed to the more common method of surrounding each detector in a cadmium shell [6]. 


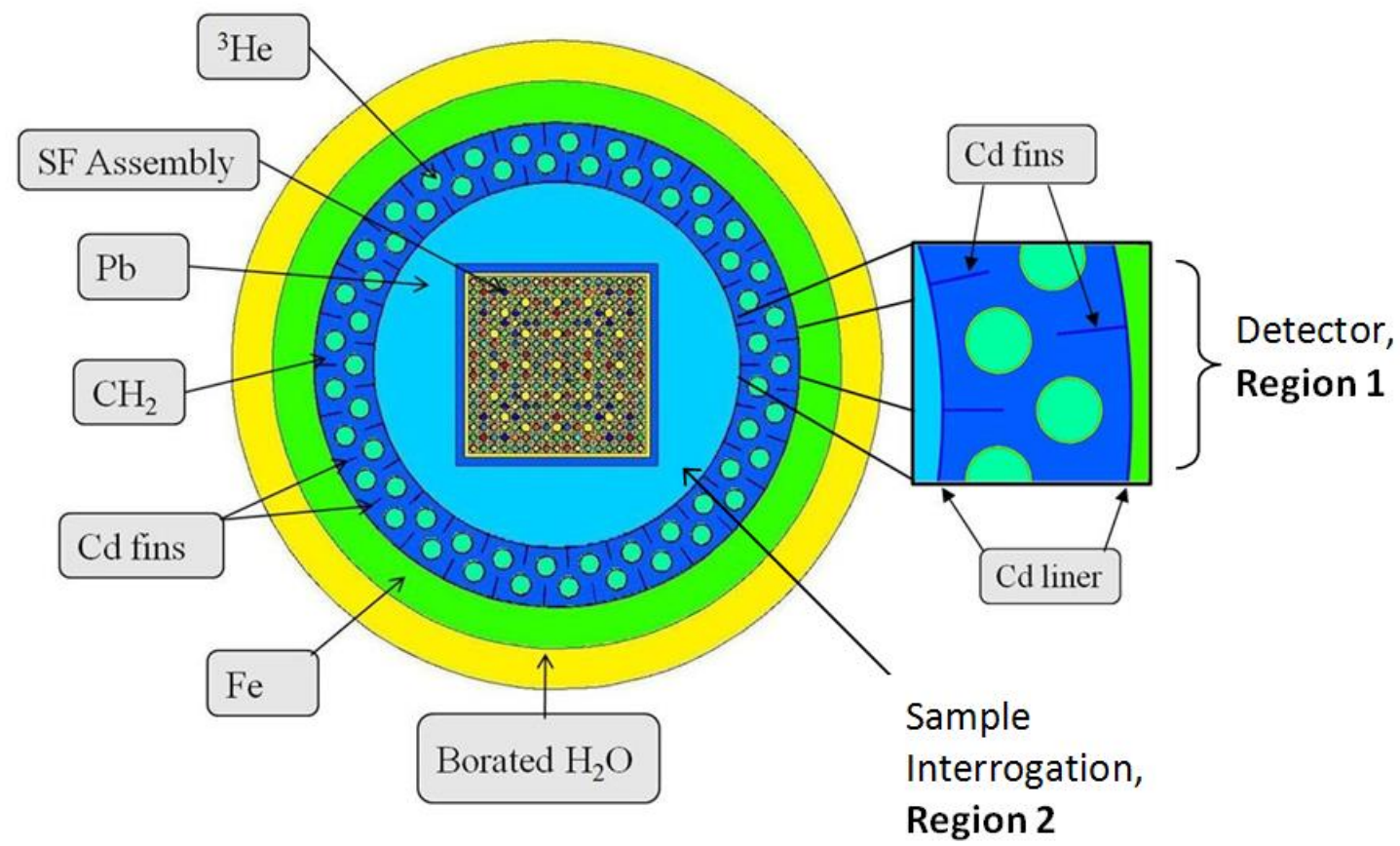

Figure 1-1. Cross-section of the original DDSI cylindrical design. Detector efficiency is $13 \%$ and die-away time is $22 \mu \mathrm{s}$.

Lead shielding was used to reduce the gamma dose to the ${ }^{3} \mathrm{He}$ tubes in the detector region. The primary goal of the design was to have a detector die-away shorter than that of the spent fuel being measured. The importance of the short detector die-away time will be discussed further in Section 1.5 .

Menlove et al. also proposed that the time-correlated distribution of neutron detection times would provide a measure of both the spontaneous fission (SF) rate and the induced fast-neutron fission (FF) rate. The visual representation of this concept is shown in Figure 1-2. [7]. The die-away time of neutrons from the fastest processes (SF and FF) was limited by the properties of the detector. The neutrons from thermal neutron-induced fission, however, would die-away significantly slower than the characteristic detector die-away and their die-away time would therefore be observable. Therefore, the publication proposed that the early doubles gate following the trigger (i.e. time zero in the time correlated distribution) should allow for measurement of the ${ }^{244} \mathrm{Cm}$ mass and the late doubles gate should allow for measurement of fissile mass. This concept was however modified in later work to account for the fact that the early time-window contains data not only from SF and fastfission events, but also from induced fission events [8,9]. 


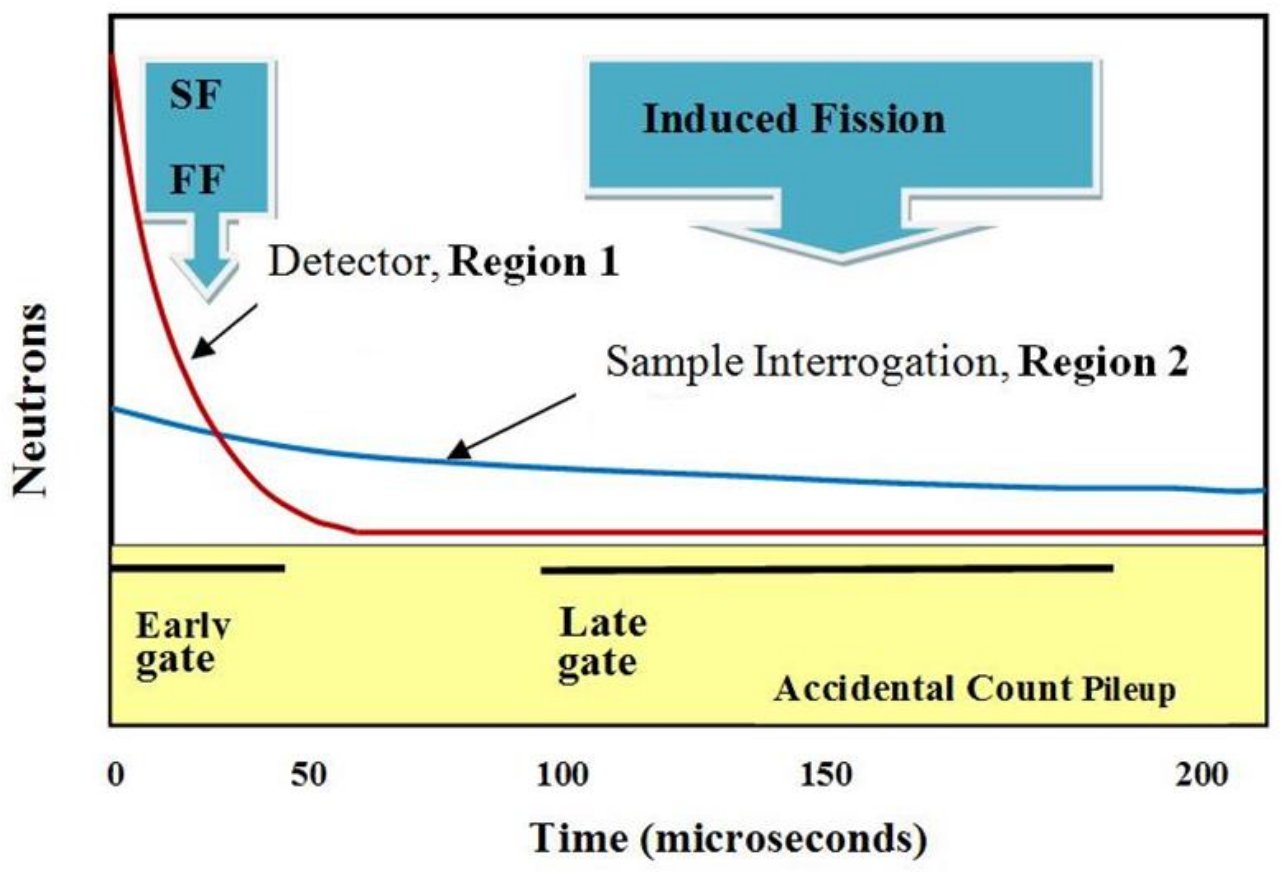

Figure 1-2. Conceptual time correlation distribution indicating data from different regions and early and late gate timing [7].

Much of the early research conducted on DDSI utilized the system design in Figure 1-1. In the NA241 report prepared by Schear et al. [10], the capabilities of the instrument were explored primarily with shift-register gate analysis. Monte Carlo simulations were used to evaluate the instrument response to parameters such as initial enrichment, burnup, cooling time, effective ${ }^{239} \mathrm{Pu}$ mass, and multiplication. The ratio of doubles in the late or early gate over singles rate $(\mathrm{D} / \mathrm{S})$ and the ratio of doubles in the late gate over doubles in the early gate $(\mathrm{L} / \mathrm{E})_{\mathrm{D}}$ were the primary characteristic monitored, and were used to determine multiplication and other parameters. The drawback of these methods of analysis is the dependency of the contribution of neutrons from $(\alpha, n)$ reactions, which must be corrected for in order to accurately determine multiplication.

The system proposed in [3] was redesigned by Belian et al. in 2012 maintaining the same physical motivations but changing to a three-sided design to accommodate a facility request for side-entry fuel measurements [11]. The design contained $57{ }^{3} \mathrm{He}$ tubes at 6 atm pressure and embedded in polyethylene. Lead was incorporated again to reduce the gamma dose and any extra polyethylene was removed to reduce the detector die-away time. The 3-sided design is shown in Figure 1-3. 


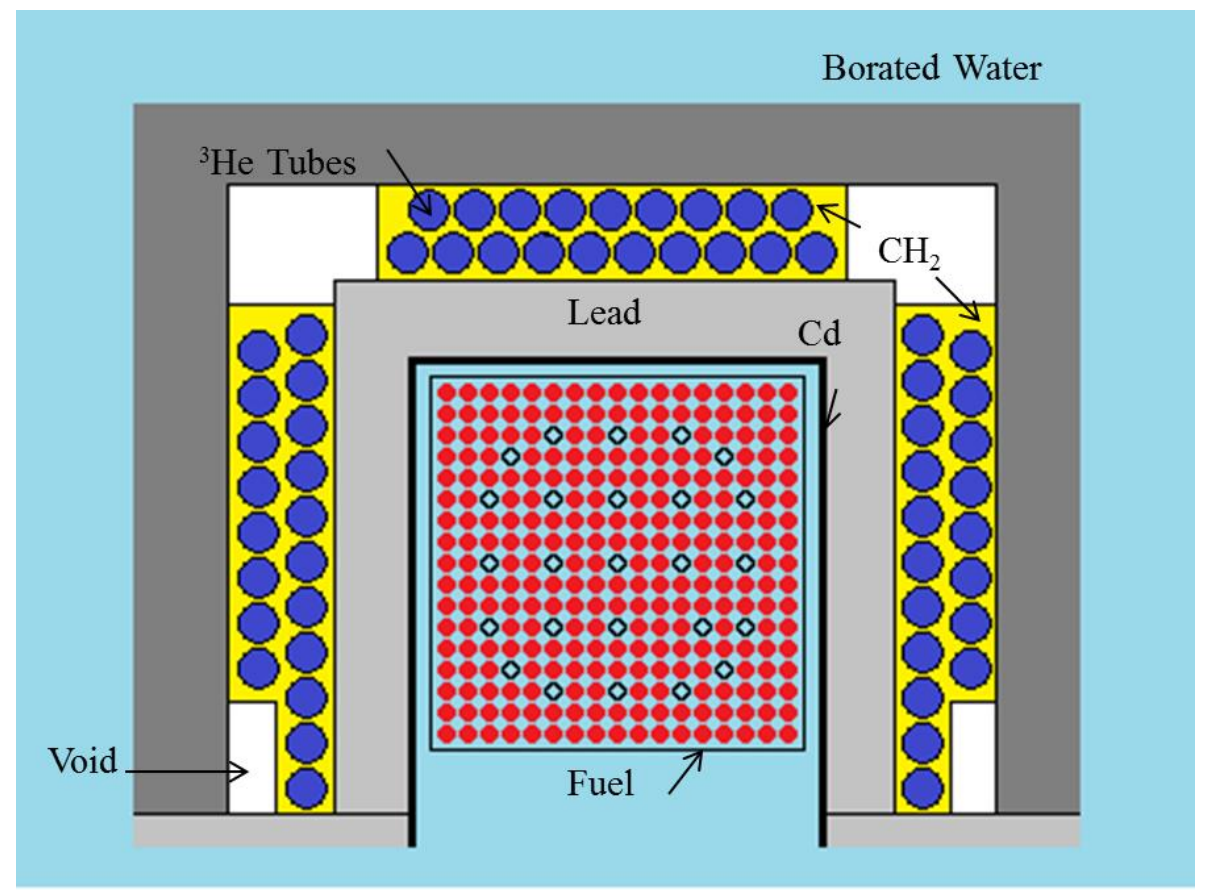

Figure 1-3. Cross-section of the three-sided DDSI design. Detector efficiency is $10 \%$ and dieaway time is $15.6 \mu \mathrm{s}$.

Effort was made to maintain the performance characteristics of the original design by incorporating cadmium lining into the detector pods instead of cadmium fins to keep the die-away time low. The measurement plan was eventually changed to incorporate a new facility that preferred a top-entry assembly measurement because the same method is used to put spent assemblies into dry casks. With this other consideration as well as the benefit of a symmetric detector system, the final DDSI instrument design was developed primarily by the author of this thesis as shown in Figure 1-4. 


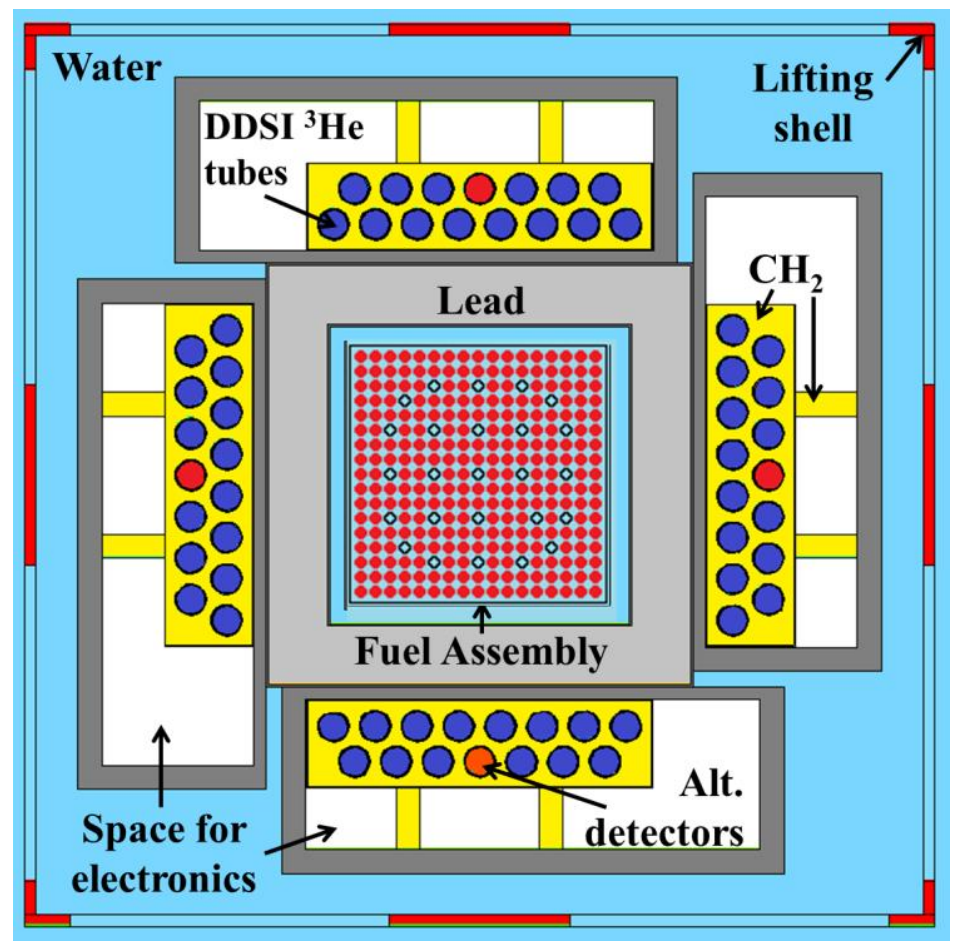

Figure 1-4. Cross-section of the final, four-sided DDSI design. Detector efficiency is $11.7 \%$ and die-away time is $19 \mu \mathrm{s}$.

The final design maintains the characteristics of the three-sided design but with the addition of the fourth side and slightly larger spacing between detectors in order to spread them out evenly between the four detector pods. Figure 1-5 shows the instrument with components labeled corresponding to descriptions in Table 1-I. Four independent pods are used that each contain their own pre-amplifiers and electronics and utilize $6 \mathrm{~atm}{ }^{3} \mathrm{He}$ tubes embedded in polyethylene to detect primarily thermalized neutrons. Thermal neutrons interact in the ${ }^{3} \mathrm{He}$ through a ${ }^{3} \mathrm{He}(\mathrm{n}, \mathrm{p}){ }^{3} \mathrm{~T}$ reaction which generates a proton/triton pair. Free electrons and ions are produced by ionization along the proton and triton tracks, and the number of charge carriers is amplified by the applied high voltage (HV) field. The resulting signal is then processed in amplifier/discrimination electronic circuits leading to creation of a transistor-transistor logic (TTL) output pulse for each (n, p) reaction. The large number of detectors provides good detection efficiency while individual amplifiers for each detector tube serve to reduce the instrument deadtime.

Three National Instruments (NI) data acquisition cards are assembled in a mini-rack crate $(61 \mathrm{~cm}$ deep, $49 \mathrm{~cm}$ wide). The NI cards have 32 channels each. Power is provided via a LANL built power supply (PXI) which provides high voltage to multiple detectors with independent control of each 
voltage. A PXI low voltage supply is used to provide $5 \mathrm{~V}$ and $+/-12 \mathrm{~V}$ to power detector amplifiers and other equipment. Coaxial cables are fed from each pod through sealed, waterproof conduit to the data acquisition system outside the pool.

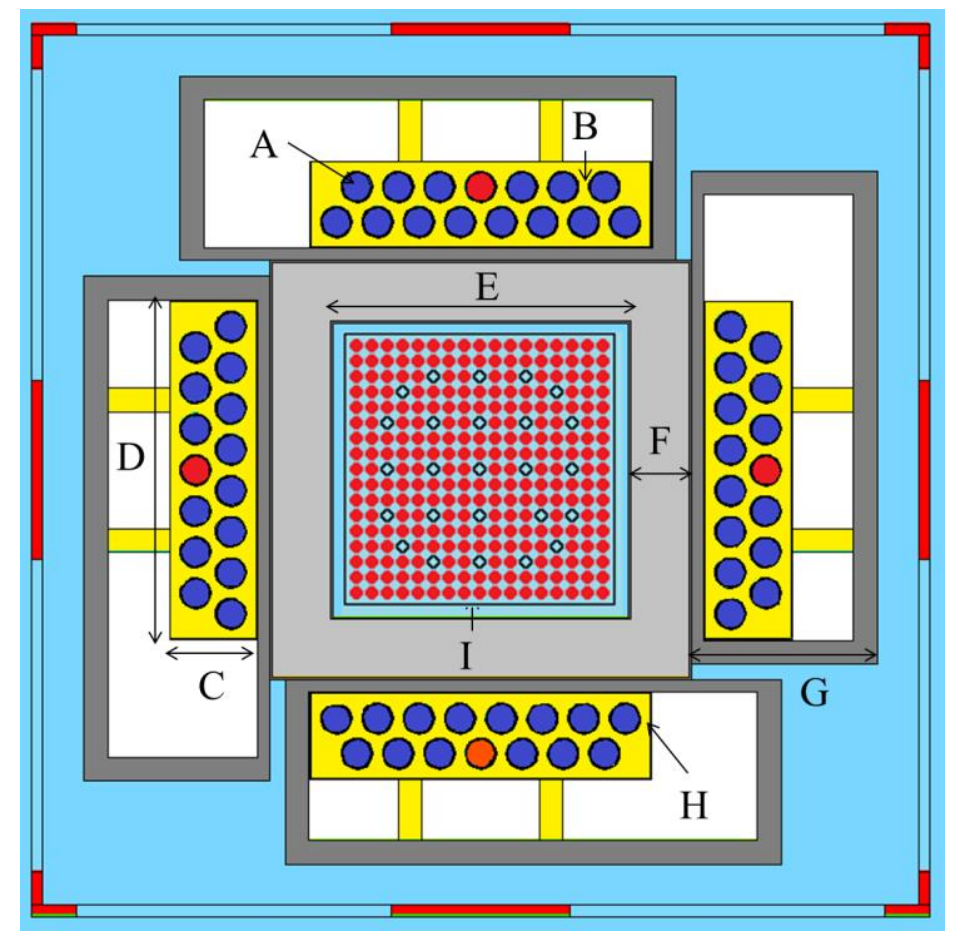

Figure 1-5. Final DDSI design with component measurements listed in Table 1-I.

Table 1-I. Dimensions of final DDSI simulated system design

\begin{tabular}{|c|c|c|c|}
\hline & Component & Measurements [cm] & Additional Specifications \\
\hline A & ${ }^{3} \mathrm{He}$ Detector & $\begin{array}{c}\text { Diameter }=2.54 \\
\text { Active length }=40.00\end{array}$ & 6 atm pressure \\
\hline B & Detector pitch & 3.5 & \\
\hline $\mathrm{C}$ & $\mathrm{CH}_{2}$ & $\begin{array}{c}\text { Thickness }=7.31 \\
\text { Height }=44.4\end{array}$ & Density $=0.96 \mathrm{~g} / \mathrm{cm}^{3}$ \\
\hline $\mathrm{D}$ & $\mathrm{CH}_{2}$ & Length $=28.81$ & \\
\hline $\mathrm{E}$ & Lead & $\begin{aligned} \text { Width } & =25.40 \\
\text { Height } & =62.90\end{aligned}$ & Density $=11.34 \mathrm{~g} / \mathrm{cm}^{3}$ \\
\hline $\mathrm{F}$ & Lead & Thickness $=5.00$ & \\
\hline G & Steel enclosure & $\begin{array}{c}\text { Width }=15.54 \\
\text { Inner thickness }=1.00 \\
\text { Outer thickness }=2.00\end{array}$ & $\begin{array}{c}\text { Density }=8 \mathrm{~g} / \mathrm{cm}^{3} \\
\text { SS } 304\end{array}$ \\
\hline $\mathrm{H}$ & Cd lining surrounding poly & Thickness $=0.05$ & Density $=8.65 \mathrm{~g} / \mathrm{cm}^{3}$ \\
\hline $\mathrm{I}$ & Assembly & $21.4 \times 21.4$ & \\
\hline
\end{tabular}


With the final DDSI instrument design came a new approach to data storage and analysis which utilized list-mode data collection and production of Rossi-alpha distributions (discussed in detail in chapter 2). The benefit of this approach is that the ability to perform shift-register analysis is not lost or limited, and die-away time and spectral analysis can be added to observe the exponential components of individual RADs. List-mode data collection is considered the most flexible and attractive approach for a neutron coincidence counting technique [12], as it allows for analysis of data at different time domains. Additionally, it has proved sufficient for list-mode to be the primary mode of data collection for DDSI. The data in different time domains contain information about the spontaneous and induced fission within the assembly through their relative magnitudes and die-away times.

\subsection{NDA Applications}

The NDA techniques within the NGSI-SF program were developed with several applications in mind, the majority of which support safeguards goals [13]. These include recovery from loss of continuity of knowledge $(\mathrm{CoK})$, termination of safeguards at geologic repositories, input accountability at reprocessing facilities, deterrence of diversion, and non-safeguards applications such as optimizing assembly selection for reprocessing.

When an assembly's integrity is in question in the case of a loss of CoK, one would like to have reliable methods to confirm that the assembly has not been tampered with and reestablish CoK on the item. NGSI NDA instruments should therefore have an improved capability to detect assembly alterations because they assess characteristics such as isotopic and elemental masses and item multiplication as opposed to total neutron and gamma emissions alone as with the Fork detector [14].

Safeguards at permanent geologic repositories are essential because of the finality of the accounting that is performed. When an assembly is buried, the material it contains is assumed to be irretrievable. If the accounting is incorrect and less material is buried than documented, the difference is then unaccounted for and that material may travel outside the safeguards regime without regulator knowledge. Direct mass accountancy and assurance of non-diversion is necessary to prevent this scenario, and those are two main goals of NDA instruments. 
Shipper-receiver difference (SRD) is determined for spent fuel assemblies that have been shipped to reprocessing facilities. This is most accurately done by assaying the assembly before and after it is shipped, however this is not always the method used. In fact, the majority of the time the SRD is determined by comparing burnup code calculations performed at the reactor facility to measurements made at the reprocessing facility. Incorporating NDA techniques at the shipper end of the process would provide more accurate SRD comparisons and could also provide faster plutonium accountancy at the reprocessing facilities.

The active development of NDA techniques and their implementation into nuclear facilities in itself acts as a deterrent to those who may want to illicitly alter or divert nuclear fuel assemblies. International safeguards are strengthened as more measurement techniques are integrated into routine shipping, storage, and reprocessing activities. The NGSI NDA instruments could provide a particularly strong deterrent as they are more penetrating and comprehensive than existing techniques, as discussed in the next section.

Finally, the technology developed can help facilities to operate more efficiently by determining burnup credit so that the process in which fuel is used, reloaded, and stored may be optimized. Heatdetermination, which is not a direct capability of any of the NGSI-SF researched technologies but is possible through burnup measurements coupled with simulation and benchmark data, could also help for repository placement determination.

This thesis focuses on the characterization of spent nuclear fuel with an NDA instrument based on the novel DDSI technique. It is the conclusion of this thesis that the DDSI instrument is capable of characterizing spent fuel with sufficient accuracy and the ways in which this can be accomplished are explained and documented in Chapter 5.

\subsection{Comparison of DDSI Method with Other NDA Techniques}

The NGSI project came into existence to improve the capabilities of the current NDA technologies. In the present, it is the fork detector (FDET) that is the primary work horse of EURATOM and the IAEA for spent fuel characterization [15], however it has since been shown that it is not the most suitable instrument in certain circumstances [16]. In addition, the FDET cannot be considered an 
independent verification technique because the results rely heavily on data provided by the operator including burnup and cooling time [17]. The FDET response is plotted against the Operator's declaration and the inspectors check for consistency within the group of assemblies measured.

The Cherenkov Viewing Device (CVD) in its various forms has been in use by the IAEA for several decades. It has been proposed as a tool for measuring partial and gross defects. However a major barrier exists which is that murky water or a deep storage pool can make the instrument unreliable. It is also limited in the range of burnups and cooling times it can measure because of the necessity of certain signal strength [18].

Safeguards MOX Python (SMOPY) is currently considered one of the more advanced spent fuel NDA characterization techniques [19]. It employs total neutron counting in combination with gamma spectroscopy to characterize spent nuclear fuel. However, the major deficiency in any technique that relies on gamma spectroscopy is the limited range of gammas in fuel due to selfshielding and the effect of Compton scattering in water. An asymmetric burnup or diversions from the center of the assembly make gamma spectroscopy-based techniques difficult to rely upon. NGSI has sought to improve upon these three standard techniques by combining new technologies to both characterize spent fuel assemblies and detect missing or illicitly substituted pins simultaneously.

The original 14 techniques to be investigated within the NGSI Spent Fuel NDA project included: Passive Neutron Albedo Reactivity (PNAR) [20], X-ray Fluorescence (XRF) [21], Passive Gamma (PG) [22], Neutron Multiplicity (NM) [23], Differential Die-Away (DDA) [24], Delayed Neutron (DN) [25], Delayed Gamma (DG) [26], ${ }^{252} \mathrm{Cf}$ Interrogation with Prompt Neutrons (CIPN) [27], Assembly Interrogation with Prompt Neutrons (AIPN) [28], Self-Interrogation Neutron Resonance Densitometry (SINRD) [29], Differential Die-Away Self-Interrogation (DDSI) [3], Lead Slowing Down Spectrometer (LSDS) [30], Neutron Resonance Transmission Analysis (NRTA) [31], and Nuclear Resonance Fluorescence (NRF) [32]. The techniques were down-selected based on several key factors including simplicity, maturity, and robustness [13]. However the review was held in the rather early stages of the investigation of these techniques and their capabilities have since been proven that were not believed to be possible during the review. For example, it was assumed that DDA and DDSI could not determine total elemental plutonium content, but that his since proven to be incorrect [33,34]. The original review committee eliminated certain techniques such as XRF based on low penetrability, and others such as LSDS based on high complexity. Neutron 
measurement techniques took precedence because of the ability to assay an assembly uniformly without having to assume its homogeneity. The result of the review was five detector systems (PNAR, SINRD, CIPN, DDSI, and DDA) to be considered in combination with the more simple counting techniques: PG, TN, and DG. These techniques can be easily integrated into the five systems because they only require detectors which may already be an inherent part of the detector system.

DDSI was found favorable by the review committee because it is a passive technique unlike CIPN and DDA. However, it was also expected to have an intermediate cost, as opposed to CIPN PNAR and SINRD which were expected to be less expensive. DDA was anticipated to be generally more expensive because of the neutron generator required.

\subsection{Neutron Coincidence Counting}

The DDSI instrument utilizes neutron coincidence counting in order to characterize the fissioning sources within the system. Any fission event will release multiple neutrons the vast majority of the time, with a different average number of neutrons depending on the isotope, fission type (i.e. spontaneous or induced), and incoming neutron energy when applicable. These fission neutrons, which are released simultaneously, are then able to be differentiated from background neutrons such as $(\alpha, n)$ that are emitted randomly and thus not correlated in time. A coincidence is defined as two neutrons arriving within a specified time window of one another which is typically on the order of 50-100 $\mu \mathrm{s}$. There is always an associated probability to detect uncorrelated neutrons, or "accidentals", with a coincidence counting technique, and this probability increases with the source strength. However, the contribution of the accidental coincidences can be statistically evaluated and separated from the real coincidences.

The most commonly used technique for coincident data collection is the shift register. Introduced by Boehnel in 1975 [35], the shift register operates by generating a predelay and gate for each pulse recorded, allowing for detector recovery time and enabling measurement of neutron coincidences. The shift register operates by opening an $\mathrm{R}+\mathrm{A}$ gate that captures both real and accidental neutron coincidences following a predelay of approximately 3-6 $\mu$ s [1]. Another long delay follows the pulse that opened the R+A gate and then an Accidentals Gate, or A gate, is opened as well. This gate can 
only contain accidental coincidences because the long delay is adequately long to exclude any real coincidences [36], that is, it is several die-away times after the triggering neutron was detected so that any truly coincident neutrons are no longer in the system when the A-gate is open.

It has been found that the accidentals rate in the $\mathrm{R}+\mathrm{A}$ gate can also be well-approximated by the total neutron event rate recorded and the gate width of the coincident gate:

$$
A=G T^{2}
$$

where $A=$ accidental count rate, $G=\mathrm{R}+\mathrm{A}$ gate width in units of time, and $T=$ total neutron count rate [1]. This formula allows for computation of A rather than direct measurement. Measurement of A through a shift-register circuit is still the preferred method, however, because it continuously corrects for changes in the neutron count rate in the experiment.

Neutron population die-away time in the system is a common parameter obtained from coincidence counting analysis that helps to characterize a system. The finite diffusion of the neutrons in the

polyethylene body of any ${ }^{3} \mathrm{He}$ based neutron detection system and the detection time in ${ }^{3} \mathrm{He}$ detectors determines the die-away time of the detector geometry itself. Most neutron coincidence counters have die-away times on the order of 30-100 $\mu$ s [1]. If the detector die-away time is sufficiently long, it will become the dominant and limiting time constant of the entire system. However if it is shorter than the characteristic time of the processes in the sample, the system die-away time can be affected by, for example, long fission chains, scattering, and additional moderation within the sample region. Such is the case with the DDSI instrument with a rather short detector die-away time of $19 \mu$ s and highly multiplicative spent fuel as the typical source [9].

\subsection{Principles of DDSI}

DDSI is a passive neutron measurement technique that utilizes neutrons emitted primarily from spontaneously fissioning nuclides within an assayed item as an internal source of neutrons to interrogate its fissile content. The fast SF neutrons thermalize in moderating material such as water or high density polyethylene (HDPE) surrounding the item and a certain fraction of them re-enter the material where they can induce additional fissions [3]. The self-interrogation process increases the 
characteristic die-away time of the item by extending fission chains that started from a spontaneous fission or (alpha, n) event.

When assaying commercial grade spent nuclear fuel with the DDSI instrument the SF is often dominated by ${ }^{244} \mathrm{Cm}$. Fresh fuel measurements with DDSI thus use an external ${ }^{244} \mathrm{Cm}$ driving source or replace this SF source with the more common ${ }^{252} \mathrm{Cf}$. Neutrons from ${ }^{244} \mathrm{Cm}$ or ${ }^{252} \mathrm{Cf}$ thermalize in the water surrounding the fuel and the fissile content is preferentially interrogated because of the high thermal neutron induced fission cross-section of fissile isotopes such as ${ }^{235} \mathrm{U},{ }^{239} \mathrm{Pu}$, and ${ }^{241} \mathrm{Pu}$, and relatively low cross-section of fertile isotopes such as ${ }^{238} \mathrm{U}$ and ${ }^{240} \mathrm{Pu}$. Figure 1-6 shows the neutron induced fission cross sections of the fissile and fertile isotopes as a function of energy.

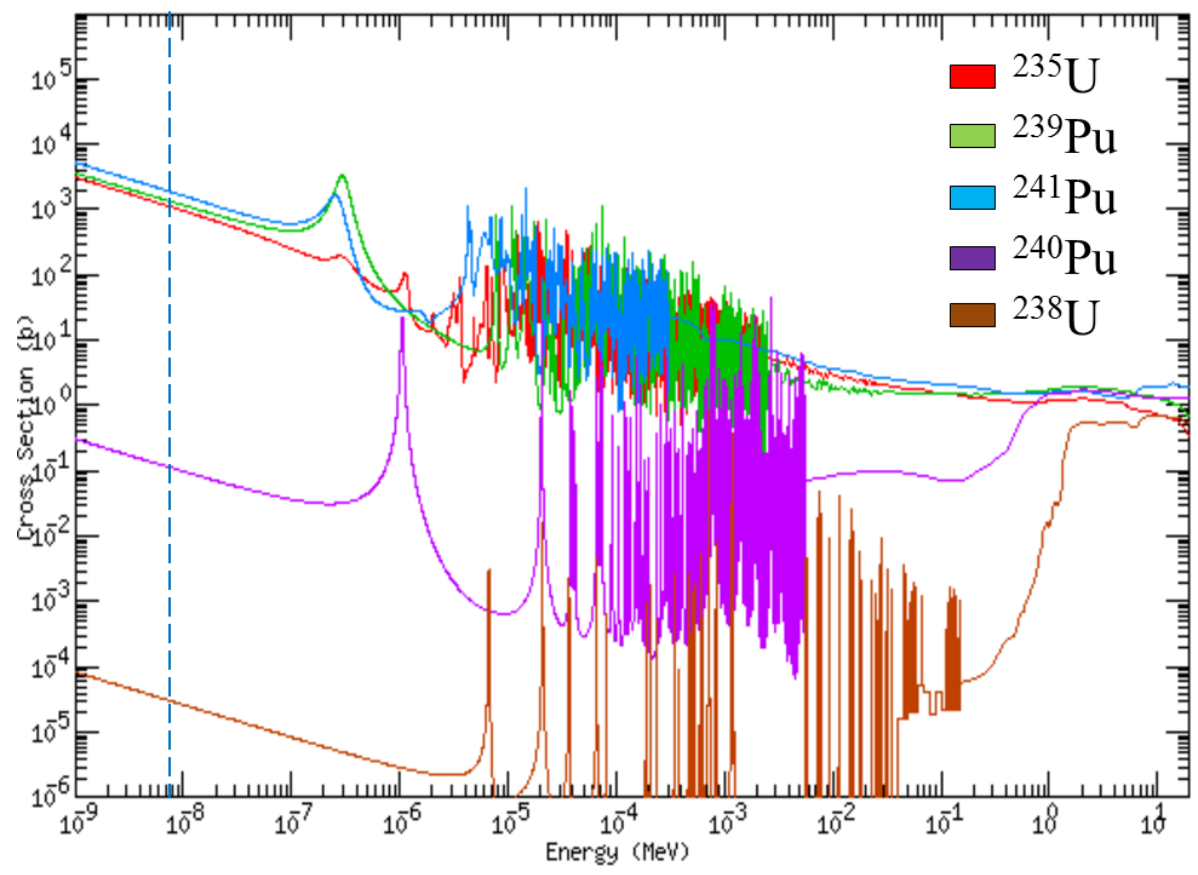

Figure 1-6. Neutron induced fission cross-sections of common fissile and fertile isotopes in SNF, from ENDF-VII. The dashed line indicates thermal neutron energy. [37]

In this way, fissile material provides a measurable neutron signal which is otherwise difficult to detect in the absence of an external, active source. The process is similar to the active interrogation concept of the Differential Die-Away technique, however, rather than an external burst of neutrons at a designated time, it is a continuous source of neutrons over the entire measurement period. Depending on the multiplication of the SFA, which reflects its isotopic composition, fission chains of various lengths develop. While the rise and fall, i.e. die-away, of the neutron subpopulation in the 
fission chain induced by any individual SF or $(\alpha, n)$ reaction cannot be measured on a case by case basis, various aspects of the evolution of the entire neutron population can be determined utilizing list-mode based neutron coincidence counting techniques. Each detected neutron is treated as a trigger and the time until the arrival of subsequent neutrons within a pre-determined time window (typically $\sim 500 \mu$ s divided into equal time bins) is added to a histogram. The next neutron in the list then becomes the trigger, and the process is repeated. This produces a Rossi-alpha distribution (discussed in detail in the next section) which allows for measurement of the die-away time of the neutron population. In the context of traditional neutron coincidence measurements the die-away time describes the decrease of the neutron population over time and is typically approximated and represented by a single exponential of the form:

$$
N(t)=N(0) e^{-t / \tau}
$$

where $N(t)$ is the neutron population at time $t$ after the neutron trigger, $N(0)$ is the initial population at the time of the neutron trigger, and $\tau$ is the time constant of the decaying neutron population and can be thought of as the mean neutron lifetime in the system, i.e. the die-away time [1]. However, due to thermalization in the source region which is typically undesirable, suppressed, or absent in traditional neutron coincidence counting approaches, the real correlation between two neutrons can be classified as belonging to one of two distinctive modes.

The first mode - fast correlation - is a result of correlation between neutrons from the same fission event, be it SF or IF, or an uninterrupted sequence of fast fission events. A fast fission event is when a neutron from one fission event induces another fission event without thermalizing first. If two neutrons are detected in coincidence from the same fission event or one fission event then a subsequent fast fission, there is a finite amount of time that those neutrons must spend traveling from the location of the fission before being captured. The neutron that arrives first will be considered the trigger neutron. The time until arrival of the second neutron will vary based on the time it takes to travel across the source region and DDSI detector and the time to thermalize in the detector before capture in one of the ${ }^{3} \mathrm{He}$ tubes. If the neutron thermalizes within the source region its lifetime becomes significantly longer and does not contribute to the fast-correlation count. A Cadmium liner around the detector polyethylene serves to prevent neutrons that thermalize in the water from entering the detector region, making the detector thus sensitive to fission neutrons only, and reducing 
the detector die-away time. Therefore the die-away time of neutrons detected from the same fission event or fast fissions is largely determined by the geometry of the ${ }^{3} \mathrm{He}$ and polyethylene which is where they are forced to thermalize. In the case of the DDSI instrument, this detector die-away is approximately $19 \mu \mathrm{s}$. A single exponential distribution can well-approximate the instrument's dieaway time only when the detector has been designed with a sufficiently uniform thickness of moderator surrounding every ${ }^{3} \mathrm{He}$ tube, as is the case with the DDSI instrument. This distribution is the fast component from the sample, and because the detector system's die-away time is considerably longer than the transit time of fast neutrons from the assembly into the detector, the total die-away of this component of the distribution is determined by the detector system.

The second mode - slow correlation - is a result of correlation among detected neutrons from different fission events within the same fission chain with at least the second fission event being induced by a thermalized neutron. In this scenario, the time between capture of the neutrons will vary significantly more than in the case of a pair of neutrons from the same fission event or an uninterrupted sequence of fast fissions. If, for example, the trigger neutron comes from a fission event that released a second neutron that thermalized and induced another fission event, the neutron released from the subsequent fission event could be detected relatively close in time after the first neutron. If, however, that second fission neutron set off a long fission chain containing multiple additional fission events before the subsequent neutron was captured, the time between detection of those two neutrons could be hundreds of microseconds. The characteristic die-away time of these neutrons is much longer $(70-120 \mu \mathrm{s})$ than the instrument's die-away time and therefore is less affected by detector setup and geometry than that of neutrons from the fast correlation. The shape of a capture-triggered distribution can still be well-approximated by a single exponential though its magnitude and die-away time constant vary widely with assembly isotopic content and water moderator conditions [1]. The slow component of a RAD can be identified in any sample where fission chains are likely to occur and have considerably longer die-away behavior than the detector; the result of a system with thermalization [38].

The RAD may contain information about both types of correlations, fast and slow, each with a significantly different characteristic die-away time constant. If a sufficiently intense slow component is present, description of the system by eq.(2), a single exponential function is not possible. 
However, the sum of two exponentials typically provides a sufficiently accurate description of the RAD obtained from the response of the DDSI instrument when assaying SFAs:

$$
N(t)=N_{0}\left[e^{-t / \tau_{f a s t}}+e^{-t / \tau_{s l o w}}\right]+A
$$

where $N(t)$ is the neutron population at time $t$ after trigger, $N_{O}$ is the initial neutron population at the time of the trigger, $\tau_{f a s t}$ is the mean lifetime (i.e. die-away time) of neutrons that did not thermalize in the source region before being detected, $\tau_{\text {slow }}$ is the mean lifetime of neutrons that thermalized and induced fission, and $A$ is the constant accidentals rate. Figure 1-7 is an example of a RAD constructed from the simulated DDSI instrument's response with both the fast and slow components displayed and accidentals subtracted.

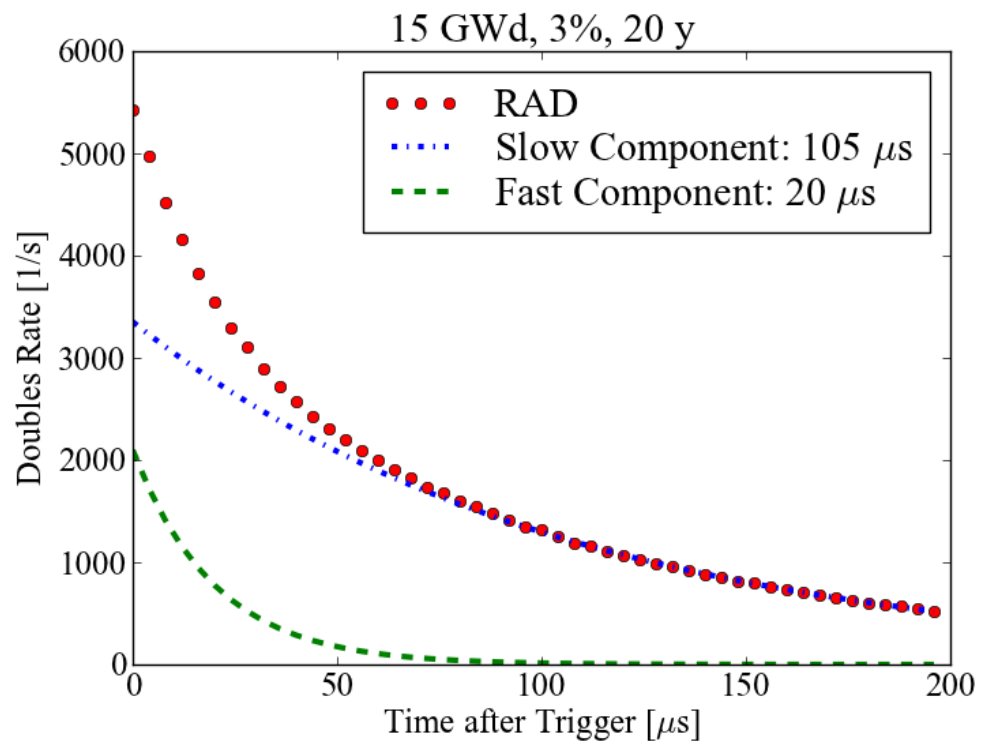

Figure 1-7. Example of a simulated RAD from assay of a SFA with $15 \mathrm{GWd} / \mathrm{tU} \mathrm{BU}, 3 \% \mathrm{IE}$, and 20 year CT. The RAD is decomposed into additive single exponentials: the fast and slow components.

The components reflect multiple characteristics of the assayed SFA. The relative magnitude of the fast and slow component as well as the slow die-away time reflects the assembly multiplication [9]. This is encapsulated in the early die-away time, which is the die-away constant from a single exponential fit from 5-52 $\mu$ s of the distribution with accidentals subtracted, shown in Figure 1-8. This relation is a novel and important finding of the thesis and will be discussed in detail in Chapter 5. 


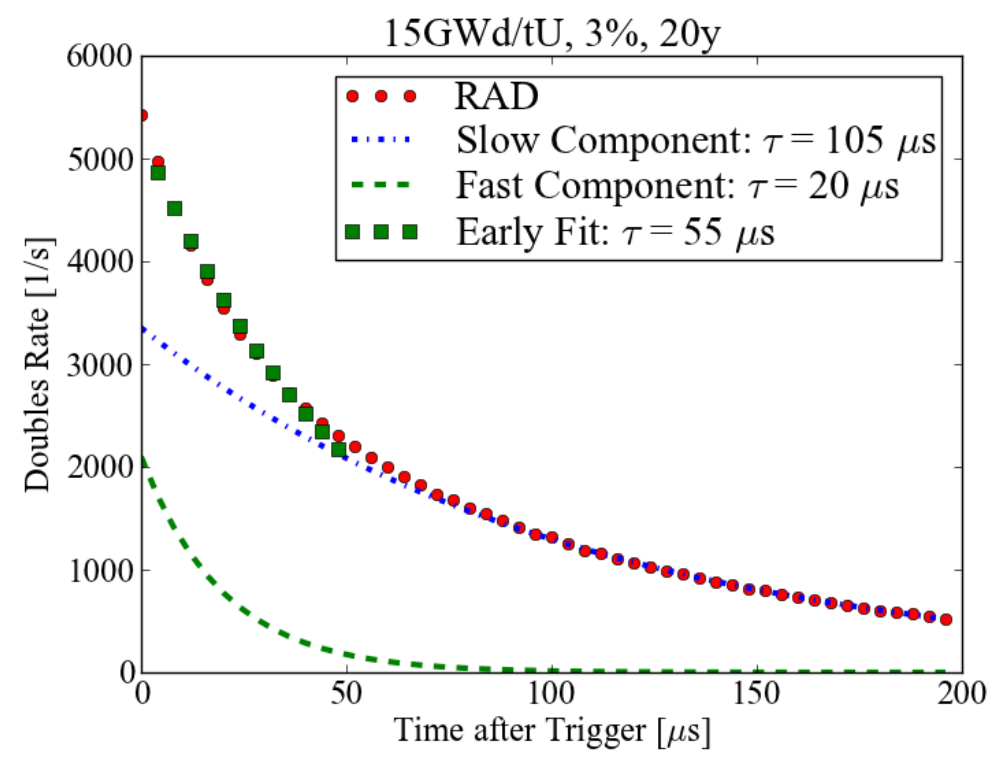

Figure 1-8. Example of a simulated RAD from assay of a SFA with $15 \mathrm{GWd} / \mathrm{tU} \mathrm{BU}, 3 \%$ IE, and 20 year CT with early die-away curve shown in green squares.

\subsection{Rossi-Alpha Distributions}

One method of characterizing an item with a neutron coincidence counting system is by producing a Rossi-alpha distribution, which is a histogram of times between triggering neutrons and detection times of subsequent neutrons in a predetermined time interval. RADs were developed originally for reactor noise analysis and have proven useful tools for flexible analysis of coincidence data as well [1]. List-mode data collection records the time of detection of each neutron by the data acquisition system. The result is a list of detection times in ascending order which is called a pulse train. Consider the following schematic pulse train depicted in Figure 1-9 for an example of how to create the RAD. The horizontal axis represents an arbitrary unit of time and the vertical lines indicate a recorded neutron capture event, i.e. a pulse.

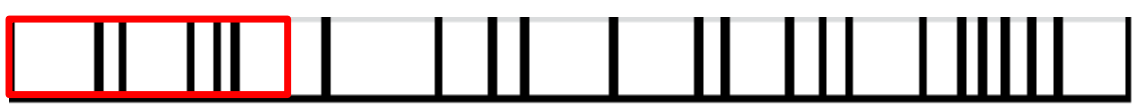

Figure 1-9. Visual representation of a pulse train, horizontal axis represents time; tick marks represent neutron detection event. Time window considered for RAD construction is shown in red.

Consider the first neutron pulse as the trigger. A time window is established a specified time after the trigger (red box shown in Figure 1-9) and the time between the trigger event and each capture 
within the window is recorded and added to a histogram of times. The second neutron pulse in the train then becomes the trigger, the window is shifted accordingly, and the process is repeated. The resulting distribution will be flat if no time correlation in neutron emission exists (such as that of an Am-Li source, an ( $\alpha, n)$-neutron source) [39]. However, if real coincident events (such as those from fission) are also present, the distribution can be described by a single exponential function as given in equation (2). While this adequately describes most coincidence counting systems, the DDSI instrument's RAD reflects two separate correlations in the distribution above the accidentals, the fast and slow components, as described in the previous section.

Though producing RADs from list-mode data is more time consuming and computationally expensive than using a shift register, obtaining the RAD itself poses benefits for measured material analysis. The shape of the RAD reflects the neutron absorbing and producing isotopes in the assembly such as fissile and fertile isotopes and neutrons absorbers. Figure 1-10 shows the change in the shape of the RAD (less accidentals) as a function of fissile/fertile ratio for a set of simplified, homogenized assemblies with primarily ${ }^{238} \mathrm{Pu}$ and ${ }^{16} \mathrm{O}$, and varying amounts of ${ }^{239} \mathrm{Pu}$ and ${ }^{240} \mathrm{Pu}$.

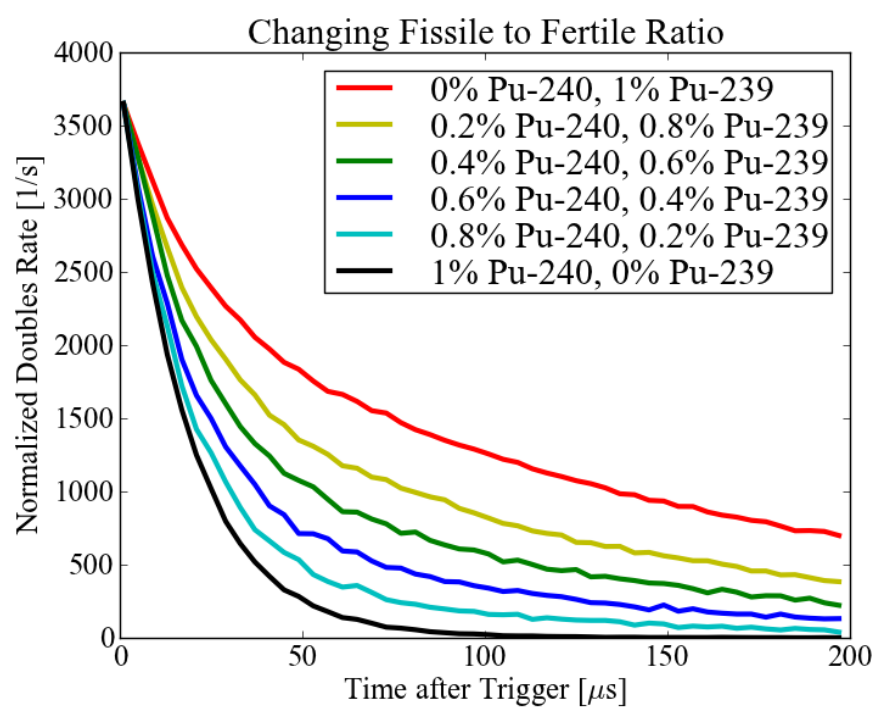

Figure 1-10. Changing simulated RAD shape as a function of fissile/fertile ratio.

As the fissile material is increased, more neutrons are added to the system in the form of long fission chains. This results in a longer average neutron lifetime, and thus a longer die-away time. The lack of fissile material and abundance of fertile (or neutron absorbing) material results in a faster, shorter die-away time representative of a short average neutron lifetime in the system. The same effect is 
observed in assemblies that are not homogenized as is seen in Figure 1-11, again with accidentals subtracted.

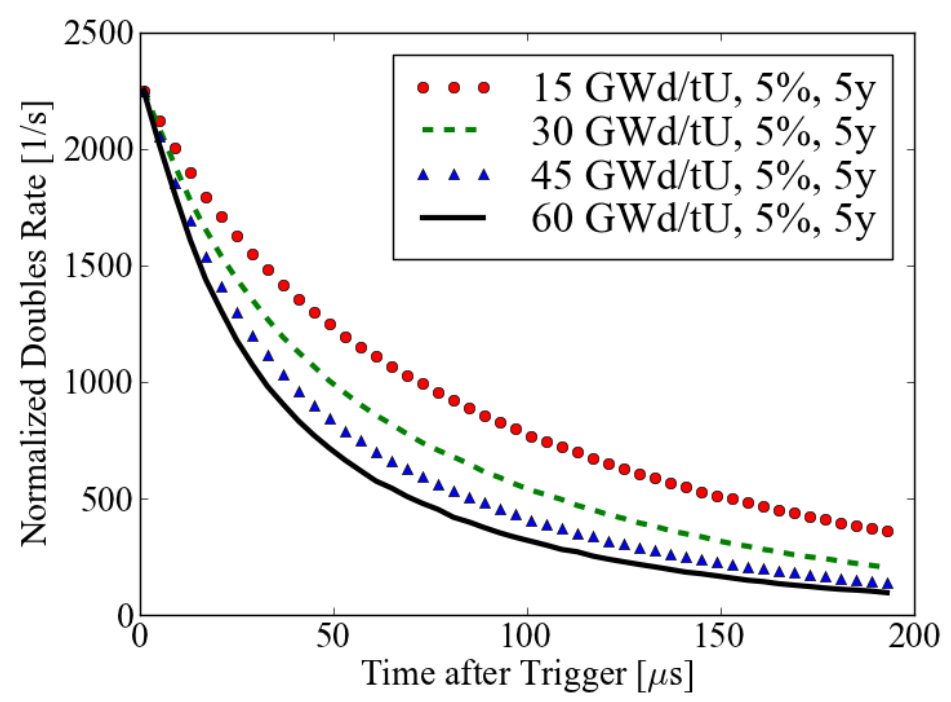

Figure 1-11. Changing RAD shape as a function of burnup in simulated spent fuel assemblies.

The more realistic assemblies in Figure 1-11 still demonstrate the same trend as the homogenous assemblies. More fissile material is present in lower burnup cases, and the result is a longer, slower die-away time. Moving forward, this unique property of RADs is utilized to characterize measured assemblies. In RADs used throughout the remainder of this thesis, the constant accidentals rate will be subtracted. 


\section{Chapter 2. InSTRUMENT HARdWARE AND DATA ACQUISITION}

The construction of the DDSI instrument was in the planning stages from 2010-2014. Several years of design optimization were conducted using MCNP resulting in the final design as discussed in 0 . The lifting mechanism design requirements were set forth by the Swedish interim storage facility in which measurements were anticipated to take place. Several images of the DDSI instrument are shown in Figure 2-1.
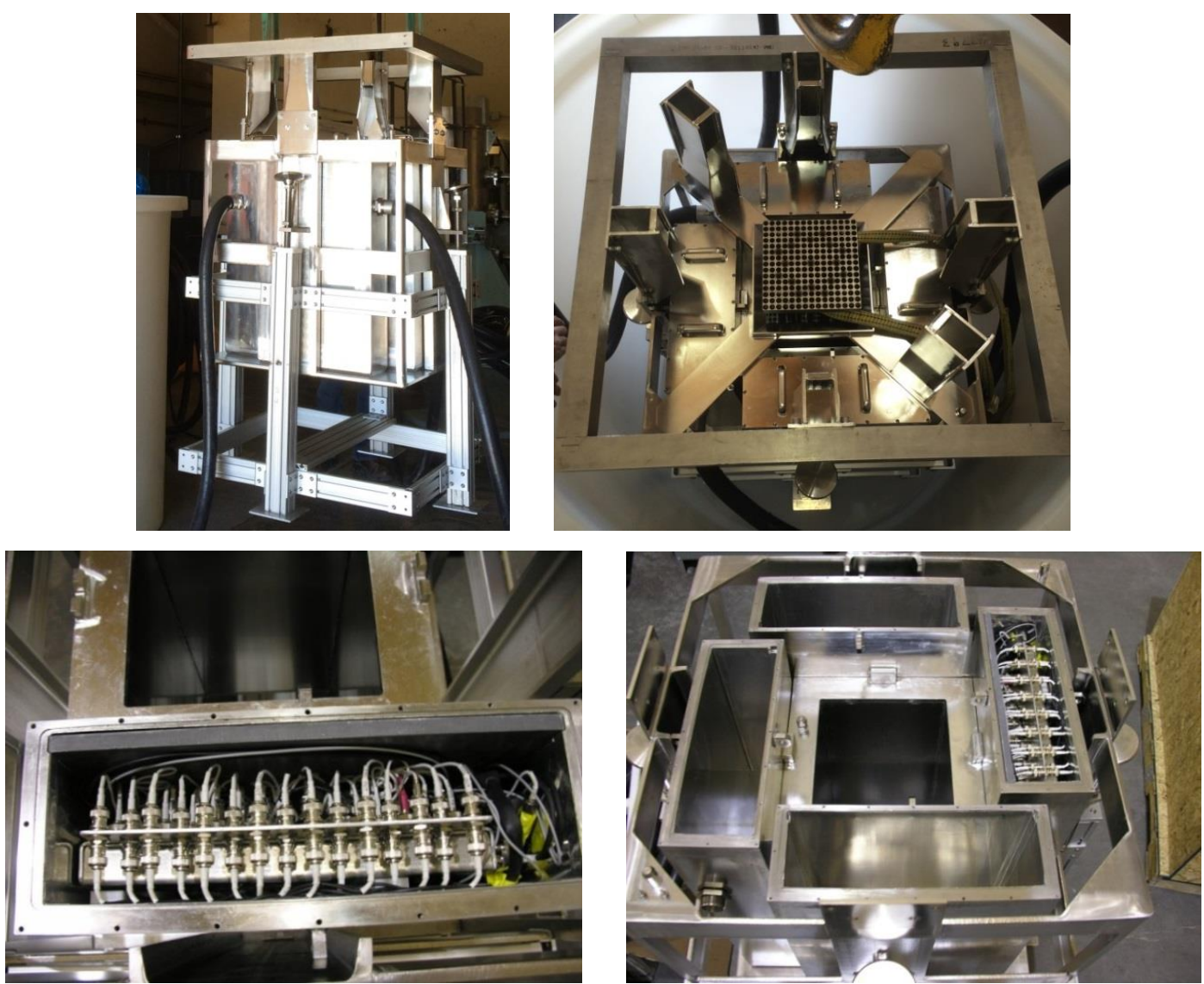

Figure 2-1. Images of the DDSI instrument. Instrument side view (top left), instrument top view with fuel rack and detector enclosures sealed (top right), open detector enclosure (bottom left), top view with three empty detector enclosures awaiting detector pods (bottom right). 


\subsection{Instrument Design Parameters}

The DDSI instrument was designed with the foremost goal of keeping the detector die-away time as low as possible. This ensured that the longer die-away time associated with thermal neutron induced fission chains in spent fuel assemblies could be observed without the timing being dominated by the detector die-away time. Reducing detector die-away was accomplished with two primary features: $\mathrm{Cd}$ lined detector regions, and a minimal amount of polyethylene surrounding the detectors. $\mathrm{Cd}$ lining served to absorb neutrons that already thermalized in the neutron source region and would thus arrive later in time. The effect of the cadmium can be seen in Figure 2-2, which displays RADs with and without the Cd lining.

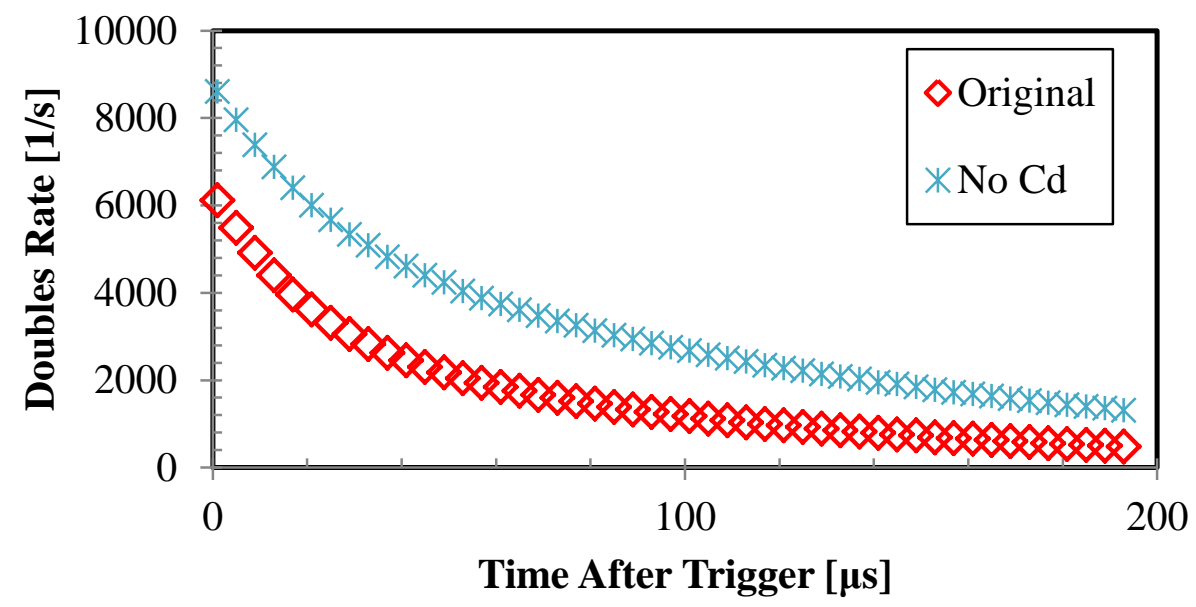

Figure 2-2. Effect of $\mathrm{Cd}$ on die-away time of a generic, simulated spent fuel assembly. "Original" has Cd surrounding all sides of the detector pods; "No Cd" has bare pods. The difference in die-away time is approximately $30 \mu \mathrm{s}$.

Reducing the die-away time had to be balanced, however, with system efficiency. The use of $\mathrm{Cd}$ and reduction of HDPE thickness surrounding the ${ }^{3} \mathrm{He}$ tubes decreased the overall detection efficiency. However, higher detection efficiency is necessary for obtaining sufficient statistics in coincidence counting. Higher gas pressure in the ${ }^{3} \mathrm{He}$ tubes increases the efficiency; therefore tubes with a pressure of $6 \mathrm{~atm}$ were selected [3]. The optimized design struck a balance between these two parameters with an efficiency of ${ }^{252} \mathrm{Cf}$ in air of approximately $11 \%$ and a die-away time of approximately $19 \mu \mathrm{s}$.

The detector system was also designed to reduce the gamma dose to the detectors as much as possible. Spent fuel emits approximately 1 million times more photons than neutrons per second. 
This high photon flux can interfere with detectors and electronics if not sufficiently shielded. $5 \mathrm{~cm}$ of lead between the assembly and detector pods and additional lead shielding above and below the detector pods was determined to be adequate shielding. This brought the gamma dose down to approximately $10-20 \mathrm{R} / \mathrm{hr}$ for the nearest detector tubes, which does not interfere with detector operation as discussed in detail in Section 2.2.2.

A series of funnels for different types of fuel assembly were designed for insertion in the center of the DDSI instrument in order to guide the assemblies into proper position. The funnels are shown in Figure 2-3.

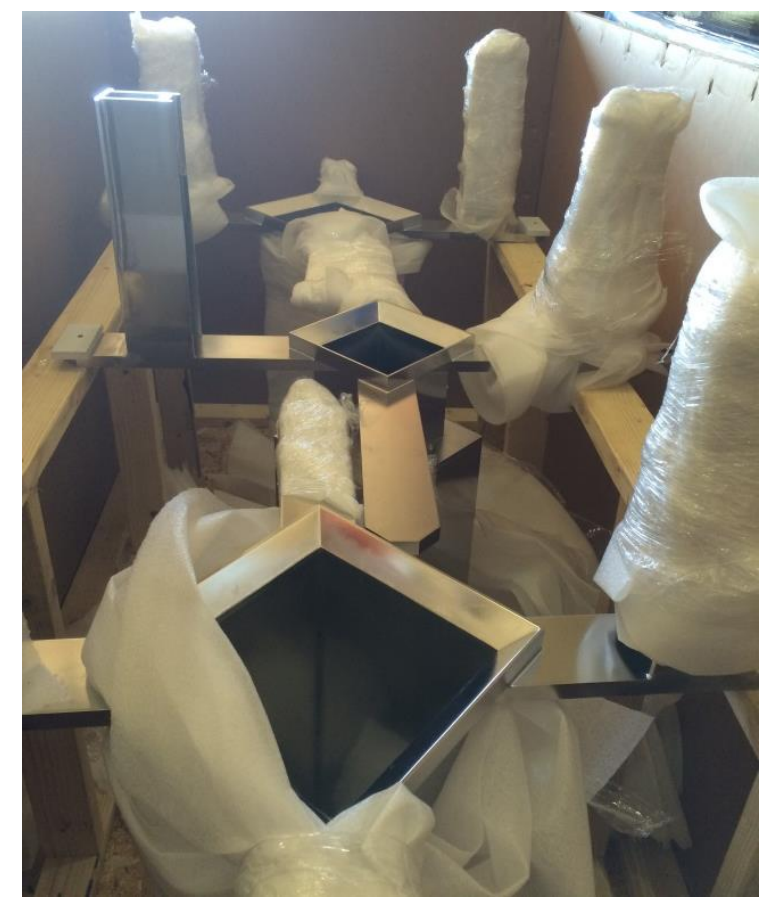

Figure 2-3. Funnels created for assembly placement in DDSI. Designed according to specifications set forth by the Swedish interim storage facility where testing of DDSI is to occur.

\subsection{Operating High Voltage of ${ }^{3} \mathrm{He}$ Detectors}

The operating high voltage was selected using the voltage plateau as well as temperature drifts and gamma dose effects. Operating at too high a voltage introduces problems with gamma pileup that are best avoided, and too low of a voltage results in an efficiency loss. The design basis of DDSI was 20 
$\mathrm{R} / \mathrm{hr}$, thus the effect of that gamma dose on ${ }^{3} \mathrm{He}$ detectors in the instrument was considered in order to determine optimal operating high voltage. Temperature could also be an issue due to the hot

environment of spent fuel storage, and thus the effect of temperature on the ${ }^{3} \mathrm{He}$ tubes and preamplifiers was investigated at different high voltages.

\subsubsection{Temperature Drifts}

Because of the heat emitted by spent fuel assemblies, temperature stability of the preamplifiers was considered in order to determine optimum operating high voltage. The preamplifiers need to be sufficiently insensitive to temperature increases experienced when the instrument is exposed to spent nuclear fuel in order for it to be reliably deployed. Though the acceptable operating temperature for ${ }^{3} \mathrm{He}$ tubes has been shown to extend as high as $200{ }^{\circ} \mathrm{C}$ [40], which is considerably higher than any anticipated environment for spent fuel, the effect of temperature on the ${ }^{3} \mathrm{He}$ tubes themselves also needed to be considered, as pulse amplitude increases and pulse resolution decreases with increasing temperature [41]. Six measurements of total neutron count rate as a function of time were conducted at $20 \mathrm{~V}$ intervals between 1600 and $1700 \mathrm{~V}$. A ${ }^{252} \mathrm{Cf}$ source was placed in the source cavity for the duration of each measurement. During each measurement, three heat lamps were turned on for a period of time of at least 3 hours. Count rates were recorded in 10 minute intervals over the length of each measurement and change in rate with heat application was observed. Three different setups were used:

Setup 1: DDSI $40 \mathrm{~cm}^{3} \mathrm{He}$ tube, 6 atm. PDT10A-HN-A111A-111 preamplifier (blue)

Setup 2: DDSI $40 \mathrm{~cm}{ }^{3} \mathrm{He}$ tube, $6 \mathrm{~atm}$. PDT-10A-HN-5V preamplifier (red)

Setup 3: Uranium Cylinder Assay System (UCAS) ${ }^{3} \mathrm{He}$ tube, $121.9 \mathrm{~cm}, 4$ atm. PDT-10AHN-5V preamplifier (green)

The room temperature was measured each time, as well as in $\mathrm{H} 1, \mathrm{H} 2, \mathrm{H} 3$, and $\mathrm{H} 4$ (shown in Figure 2-4), the UCAS preamplifier and tube, and the face of the HLNC. Using the empty slot temperature measurements it is possible to extrapolate the temperatures of S1 and S2. Henceforth, the setups are represented in plots with colors as indicated above. The three tubes were setup in three consecutive slots of an empty HLNC, shown in Figure 2-4. 


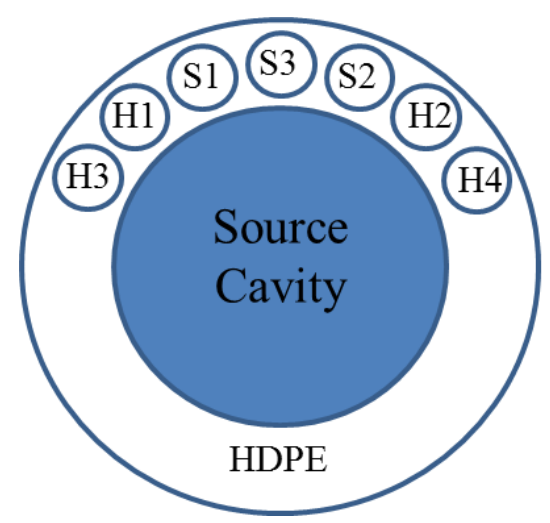

Figure 2-4. Experimental setup in HLNC well counter. S1, S2, and S3 indicate corresponding setups as discussed above. $\mathrm{H} 1, \mathrm{H} 2, \mathrm{H3}$ and $\mathrm{H} 4$ are the empty slots in which additional temperature measurements were taken.

After gain matching the three tube/amplifier setups, high voltage plateaus were recorded. Gain settings were matched by taking the ratio of the counts at 1680 to those at 1480 applied volts. HV plateaus for each setup are shown in Figure 2-5.

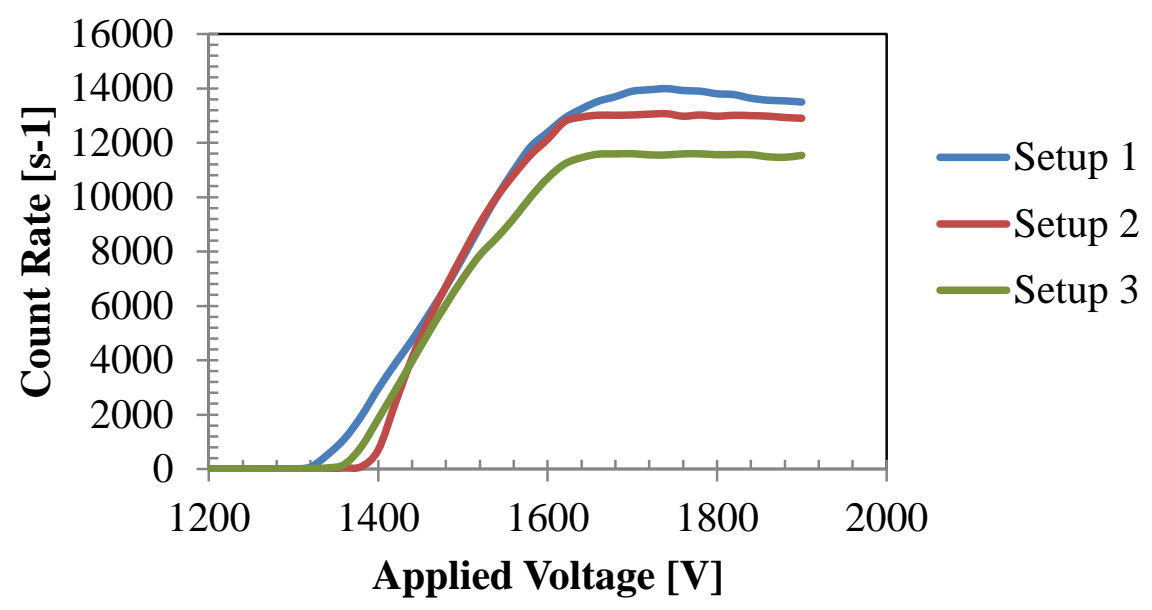

Figure 2-5. High voltage plateaus for three setups.

Once the preamplifiers were gain-matched as best possible, a variety of temperature measurements were taken before starting ${ }^{252} \mathrm{Cf}$ measurements. Assuming linear change in temperature between the slot measurement results, Table 2-I outlines the temperatures measurements in the slots and extrapolated values. The difference between $\mathrm{H} 3$ and $\mathrm{H} 1$ is added to $\mathrm{H} 1$ to find S1. Similarly, the difference between $\mathrm{H} 4$ and $\mathrm{H} 2$ is added to $\mathrm{H} 2$ to find $\mathrm{S} 2$. The adjustment values for $1660 \mathrm{~V}$ and $1680 \mathrm{~V}$ are then averaged and used for the remainder of cases. For example, if $\mathrm{H} 3$ is $38^{\circ}$ and $\mathrm{H} 1$ is $40^{\circ}$, the adjustment is $2^{\circ}$. 
Table 2-I. Empty slot measurements and extrapolations to S1 and S2. Extrapolations are done using the linear adjustment found from the difference between $\mathrm{H3}$ and $\mathrm{H1}$, and $\mathrm{H} 4$ and $\mathrm{H} 2$.

\begin{tabular}{ccccc|cccc}
\hline & H3 & H1 & $\begin{array}{c}\text { H1-H3 } \\
\text { (adjustment) }\end{array}$ & $\begin{array}{c}\text { S1 } \\
{[\mathbf{C}]}\end{array}$ & $\begin{array}{c}\text { H4 } \\
{[\mathbf{C}]}\end{array}$ & $\begin{array}{c}\text { H2 } \\
{[\mathbf{C}]}\end{array}$ & $\begin{array}{c}\text { H2-H4 } \\
\text { (adjustment) } \\
{[\text { [C] }]}\end{array}$ & $\begin{array}{c}\text { S2 } \\
{[\mathbf{C}]}\end{array}$ \\
\hline $\mathbf{1 6 6 0}$ & 38.3 & 41.3 & 3.0 & 44.3 & 39.5 & 41.4 & 1.9 & 43.3 \\
$\mathbf{1 6 8 0}$ & 36.8 & 39.8 & 3.0 & 42.8 & 39.5 & 42.7 & 3.2 & 45.9 \\
Avg. Adjustment & & & 3.0 & & & & 2.55 & \\
\hline
\end{tabular}

The average adjustment for the left side (3.0) is added to the average adjustment for the right side (2.55) to determine the total $\mathrm{S} 3$ adjustment. $\mathrm{S} 3$ is then determined by the total adjustment plus $\mathrm{S} 1$. Table 2-II shows the temperature measurements described in the paragraph above as well as adjusted temperature estimates for S1, S2, and S3 based on Table 2-I.

Table 2-II. Temperature measurements in the room, left and right slots, UCAS tube and preamplifier, and face of the HLNC for each high voltage.

\begin{tabular}{|c|c|c|c|c|c|c|c|c|c|c|}
\hline $\begin{array}{l}\mathrm{HV} \\
{[\mathrm{V}]}\end{array}$ & Time & $\begin{array}{c}\text { Room } \\
{[\mathrm{C}]}\end{array}$ & $\begin{array}{l}\text { H1 } \\
{[\mathrm{C}]}\end{array}$ & $\begin{array}{l}\mathrm{H} 2 \\
{[\mathrm{C}]}\end{array}$ & $\begin{array}{c}\text { Adj. } \\
\text { S1 } \\
{[C]}\end{array}$ & $\begin{array}{c}\text { Adj. } \\
\text { S2 } \\
{[C]}\end{array}$ & $\begin{array}{c}\text { Adj. } \\
\text { S3 } \\
{[C]}\end{array}$ & $\begin{array}{c}\text { UCAS } \\
\text { Preamp } \\
{[C]}\end{array}$ & $\begin{array}{c}\text { UCAS } \\
\text { Tube } \\
{[C]}\end{array}$ & $\begin{array}{c}\text { Face of } \\
\text { HLNC } \\
{[C]}\end{array}$ \\
\hline \multirow{2}{*}{1600} & Start & 27.3 & 27.8 & 27.8 & 27.8 & 27.8 & 27.8 & 31.2 & 27.2 & 27.6 \\
\hline & Light & 27.8 & 37.8 & 39.8 & 40.8 & 42.4 & 46.4 & 33.8 & 33 & 36.8 \\
\hline \multirow{2}{*}{1620} & Start & 27 & 27 & 27 & 27 & 27 & 27 & 27.4 & 27.4 & 27.6 \\
\hline & Light & 27.8 & 37.3 & 38.2 & 40.3 & 40.8 & 45.9 & 32.3 & 29.3 & 52.8 \\
\hline \multirow{2}{*}{1640} & Start & 27.6 & 28.2 & 28.1 & 28.2 & 28.1 & 28.2 & 30.4 & 27.6 & 27.8 \\
\hline & Light & 28.3 & 42.7 & 46.1 & 45.7 & 48.7 & 51.3 & 29.9 & 30.1 & 56 \\
\hline \multirow{2}{*}{1660} & Start & 27.7 & 28.6 & 28.6 & 28.6 & 28.6 & 28.6 & 28.9 & 27.8 & 28.4 \\
\hline & Light & 28.4 & 41.3 & 41.4 & 44.3 & 43.3 & 49.9 & 30.2 & 30.2 & 56.8 \\
\hline \multirow{2}{*}{1680} & Start & 27.8 & 28.4 & 28.4 & 28.4 & 28.4 & 28.4 & 28.4 & 28 & 28.3 \\
\hline & Light & 28.4 & 39.8 & 42.7 & 42.8 & 45.9 & 48.4 & 28.7 & 29 & 56.5 \\
\hline \multirow{2}{*}{1700} & Start & 27.3 & 27.8 & 27.8 & 27.8 & 27.8 & 27.8 & 31.2 & 27.2 & 27.6 \\
\hline & Light & 28 & 36.2 & 38.7 & 39.2 & 41.3 & 44.8 & 31 & 35 & 38 \\
\hline
\end{tabular}


Change in the count rate as a function of time with vertical lines indicating when heat lamps were turned on and off are given for each voltage in Figure 2-6 - Figure 2-11. Times of applied heat are given in the figure captions. Table 2-III shows the average and standard deviation of count rates in the stable region where heat is not affecting the count rate after approximately $50,000 \mathrm{~s}$, or $\sim 14$ hours. It also provides the maximum or minimum count rate of each setup, and the resulting maximum percent deviation from the average in the heated time region per ${ }^{\circ} \mathrm{C}$ temperature change.

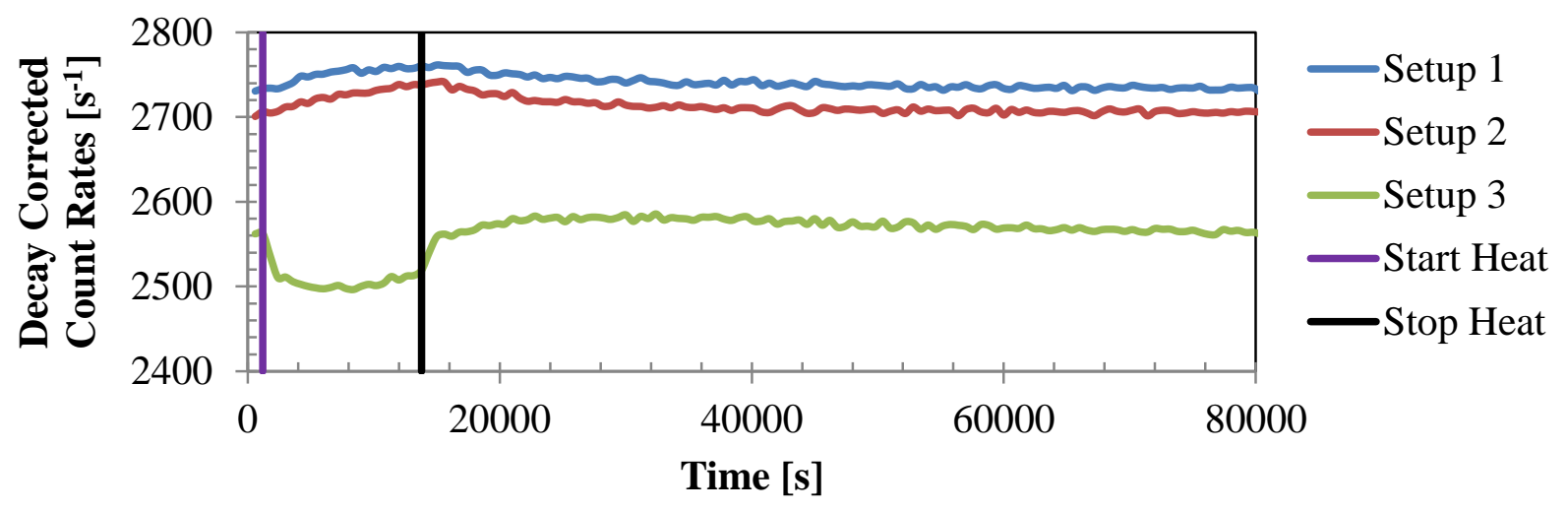

Figure 2-6. $1600 \mathrm{~V}$, heat lamp turned on at 20 minutes, removed at $3.8 \mathrm{hrs}$.

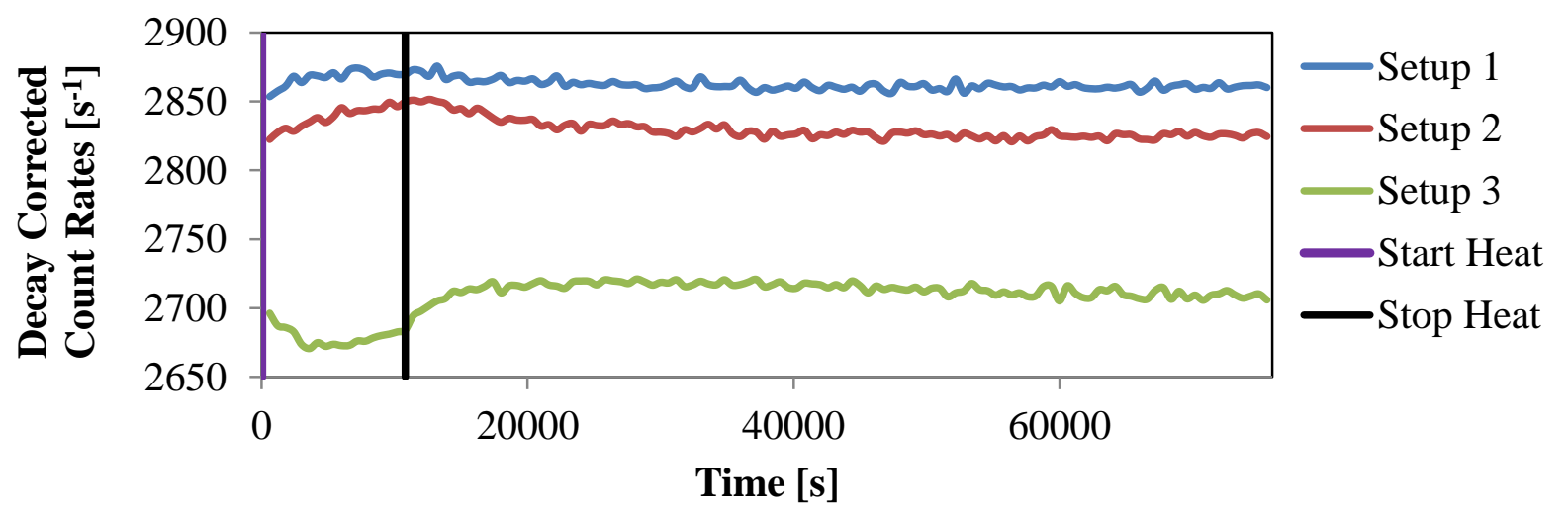

Figure 2-7. $1620 \mathrm{~V}$, heat lamp turned on at 0 minutes (start), removed after 3 hrs. 


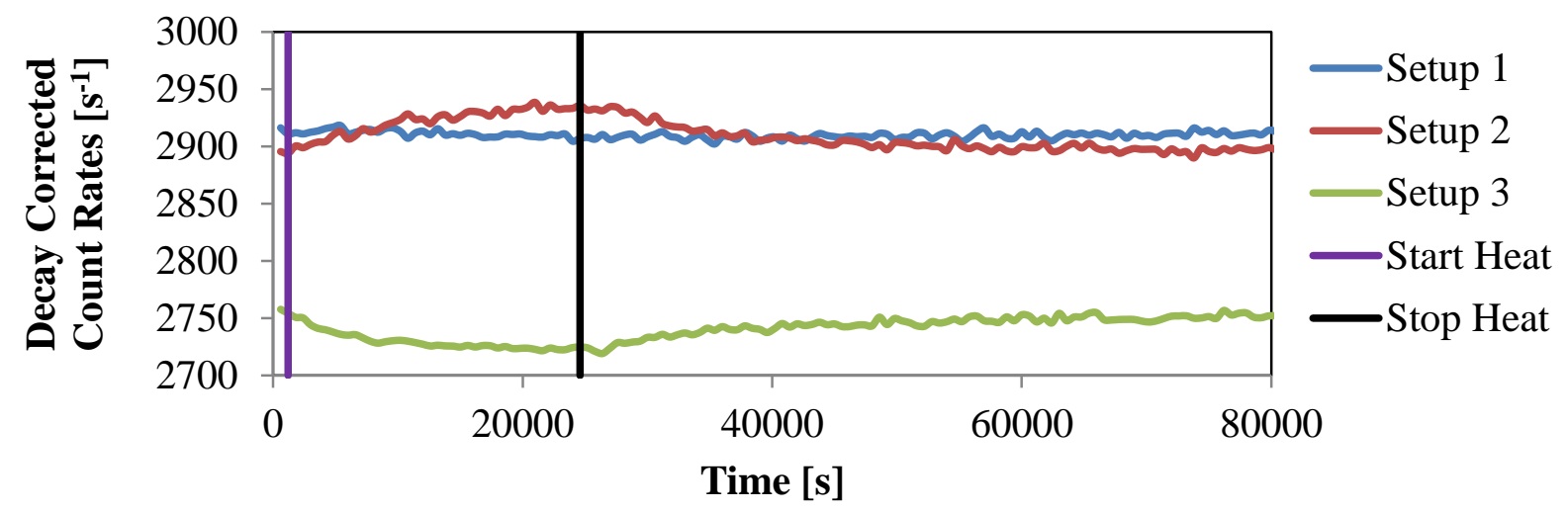

Figure 2-8. $1640 \mathrm{~V}$, heat lamp turned on at 20 minutes, removed after $6.8 \mathrm{hrs}$.

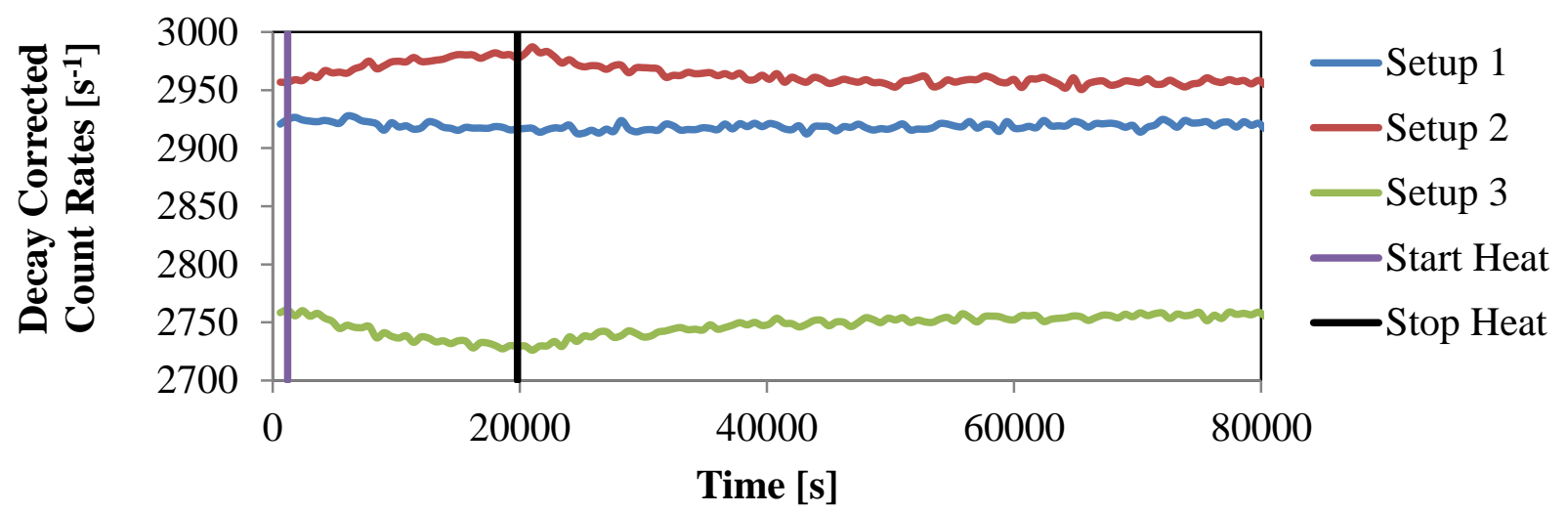

Figure 2-9. $1660 \mathrm{~V}$, heat lamp turned on at 20 minutes, removed after $5.5 \mathrm{hrs}$.

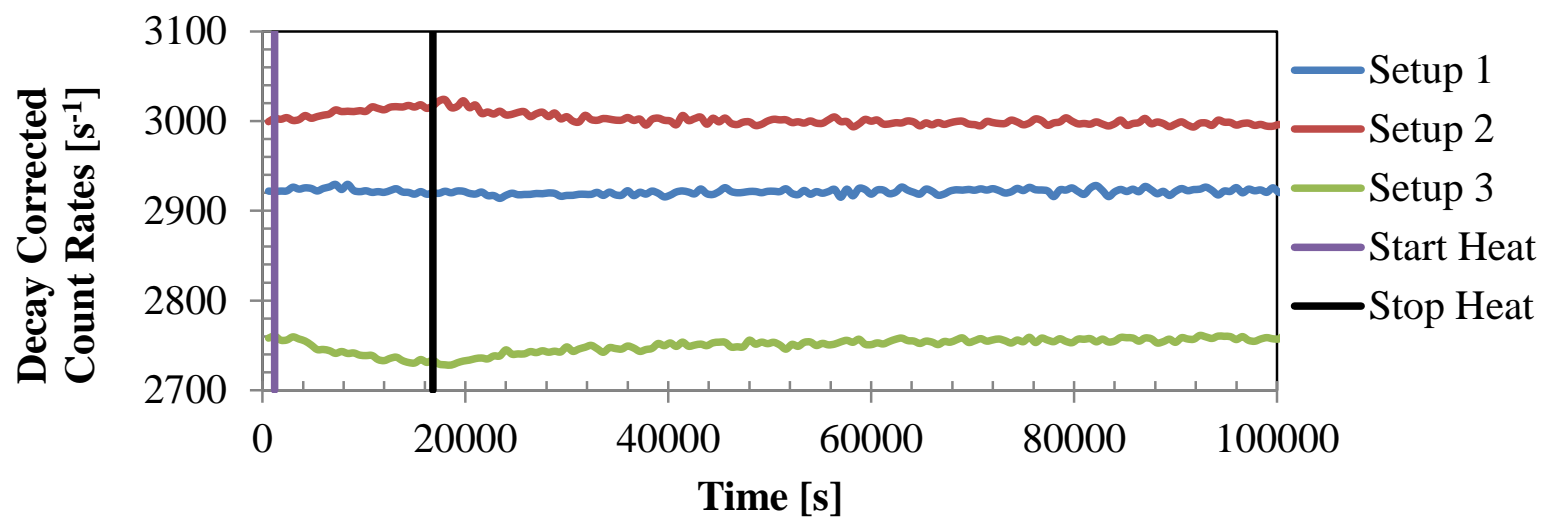

Figure 2-10. $1680 \mathrm{~V}$, heat lamp turned on at 20 minutes, removed after $4.7 \mathrm{hrs}$. 


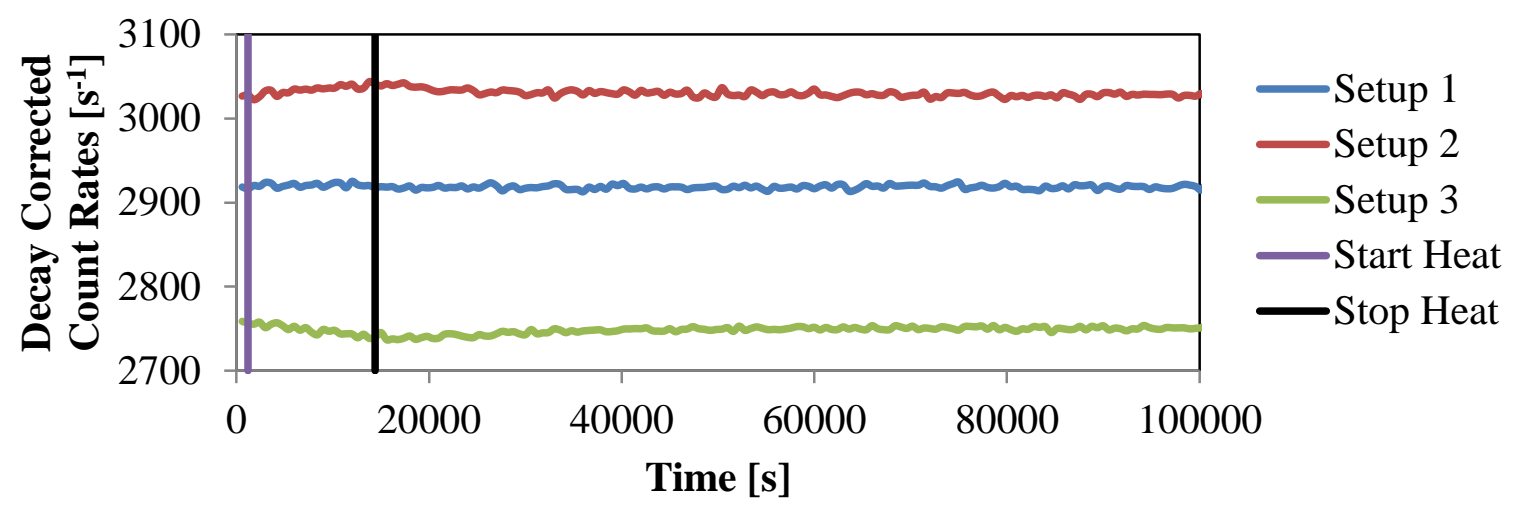

Figure 2-11. $1700 \mathrm{~V}$, heat lamp turned on at 20 minutes, removed after $4 \mathrm{hrs}$.

Table 2-III. Average, standard deviation, and maximum or minimum as well as deviation per ${ }^{\circ} \mathrm{C}$ for each case.

\begin{tabular}{ccccc}
\hline Setup & Average & Std. Dev. & Max or Min & Deviation $[\%]^{\mathbf{0}} \mathbf{C}$ \\
\hline 1600: S1 & 2734.7 & 2.0 & 2761.4 & -0.08 \\
1600: S2 & 2706.3 & 2.3 & 2741.4 & 0.09 \\
1600: S3 & 2566.5 & 4.5 & 2496.5 & -0.15 \\
1620: S1 & 2860.6 & 2.1 & 2875.6 & 0.04 \\
1620: S2 & 2824.9 & 1.9 & 2851.5 & 0.07 \\
1620: S3 & 2710.6 & 3.3 & 2670.6 & -0.08 \\
1640: S1 & 2910.6 & 2.4 & 2914.4 & 0.02 \\
1640: S2 & 2897.8 & 3.2 & 2938.6 & 0.07 \\
1640: S3 & 2750.2 & 3.0 & 2719.2 & -0.05 \\
1660: S1 & 2919.8 & 2.5 & 2927.6 & 0.02 \\
1660: S2 & 2957.0 & 2.6 & 2987.2 & 0.07 \\
1660: S3 & 2755.0 & 2.5 & 2726.1 & -0.05 \\
1680: S1 & 2922.5 & 2.3 & 2931.0 & 0.02 \\
1680: S2 & 2996.9 & 2.3 & 3023.8 & 0.05 \\
1680: S3 & 2757.6 & 2.2 & 2728.0 & -0.05 \\
1700: S1 & 2918.7 & 2.3 & 2925.2 & 0.02 \\
1700: S2 & 3027.8 & 2.5 & 3043.8 & 0.04 \\
1700: S3 & 2751.0 & 2.0 & 2736.8 & -0.03 \\
\hline
\end{tabular}


The lowest operating high voltage of $1600 \mathrm{~V}$ showed the greatest temperature sensitivity for the DDSI setup (setup 1) and should therefore be avoided. However, the other operating high voltages would be suitable for DDSI because the ${ }^{3}$ He tubes and PDT A111 preamps were mostly insensitive to temperature changes. Setup 2 with the PDT 110A performed slightly worse than the PDT A111 in setup 1 and would therefore be more sensitive to temperature changes in spent fuel measurements and is best avoided. The UCAS detector setup performed the worst, likely because of the very long tube which is more susceptible to changes in temperature. Because the DDSI setup was largely insensitive to temperature, gamma dose will be the limiting factor for operating high voltage selection.

\subsubsection{Gamma Dose}

The maximum expected gamma ray dose to the ${ }^{3} \mathrm{He}$ detectors during any commercial grade spent fuel measurement is approximately $20 \mathrm{R} / \mathrm{hr}$, and this has been the design basis for the DDSI instrument. This number was based on the most active considered assembly $(60 \mathrm{GWd} / \mathrm{tU}$ BU, $5 \%$ IE, $5 \mathrm{y} \mathrm{CT}$ ) and took into account the significant lead shielding including $5 \mathrm{~cm}$ around the assembly and additional lead blocks inside the stainless steel detector pods. Though ${ }^{3} \mathrm{He}$ detectors are not designed for measurement of gamma rays, Compton scattering of incoming photons can take place within the detector walls or fill gas $\left(\mathrm{CH}_{4}\right.$ in this case) yielding free Compton electrons which can produce a column of ionization as it moves through the detector [1]. The pulses produced by gamma rays are typically much smaller than those of neutrons and are thus not usually above the amplifier threshold. But with a high enough gamma flux, the gamma pulses can pileup and create enough ionization to produce a pulse that is over the threshold [42]. DDSI ${ }^{3} \mathrm{He}$ tubes are paired with fast Amptek A111 preamplifiers for better performance in high gamma dose scenarios. In order to quantify the benefit of the faster preamplifier and ensure quality performance in high gamma environments, gamma dose measurements were conducted with both a DDSI A111 and a PDT-110A preamplifier for performance comparison, each paired with a standard 6 atm DDSI ${ }^{3} \mathrm{He}$ tube.

The LANL ${ }^{226}$ Ra source was used to expose the detector setup with a high gamma dose to determine the optimal operating high voltage. The ${ }^{226} \mathrm{Ra}$ source produced a multi-energy gamma-ray spectrum up to approximately $3 \mathrm{MeV}$ with an average energy of $2.3 \mathrm{MeV}$. It provides a dose rate of approximately $50 \mathrm{Rad} / \mathrm{hr}$ at a distance of $10 \mathrm{~cm}$. Optimal operating high voltage will be determined by observing the effect of the gamma pileup as the high voltage is raised in the following 
experiments and determining the highest operating voltage where the pileup does not affect the count rate.

A $7.8 \mu \mathrm{Ci}{ }^{252} \mathrm{Cf}$ source was placed adjacent to the detector pod and fixed at the axial center of the ${ }^{3}$ He tubes. This provided a constant neutron source in order to determine at what dose rate gamma ray pulse pile up starts to interfere with the neutron measurement. The radium source was then placed at different distances from the detector pod to provide different gamma doses. Figure 2-12 contains a schematic of the experimental setup. Figure 2-13 shows a picture taken through the hot cell leaded window with the radium source at the $20 \mathrm{~cm}$ mark.

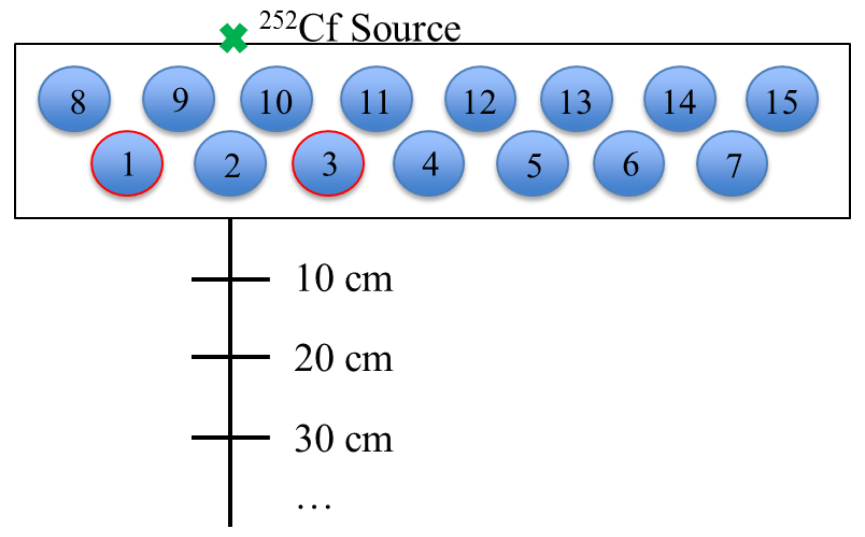

Figure 2-12. Experimental setup schematic. ${ }^{252} \mathrm{Cf}$ source was affixed to the back of the pod between the two detectors in use, marked in red. Detector 1 was paired with the PDT-110A amplifier and detector 3 was paired with the A111 amplifier. The radium source was placed in increments of $10 \mathrm{~cm}$ from the pod from $10 \mathrm{~cm}$ out to $70 \mathrm{~cm}$. 


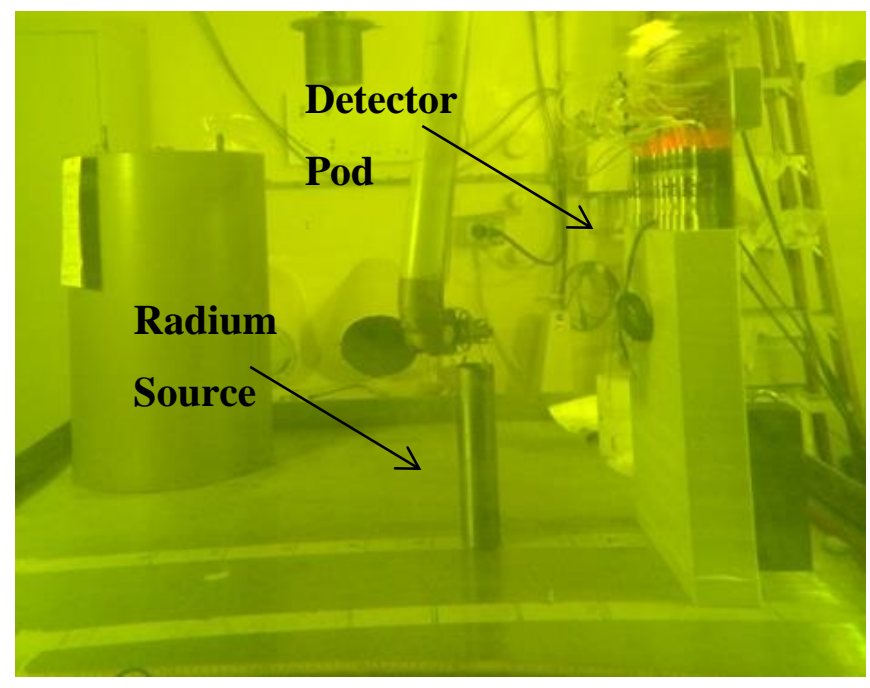

Figure 2-13. Picture taken of radium source at $20 \mathrm{~cm}$ distance.

Initially, a reference high voltage plateau was taken with the ${ }^{252} \mathrm{Cf}$ source alone. This plateau was then overlaid on the ${ }^{226} \mathrm{Ra}+{ }^{252} \mathrm{Cf}$ measurements in Figure 2-15 to demonstrate where the count rate deviates because of gamma pile up effects. The reference plateau for each preamplifier is shown in Figure 2-14.

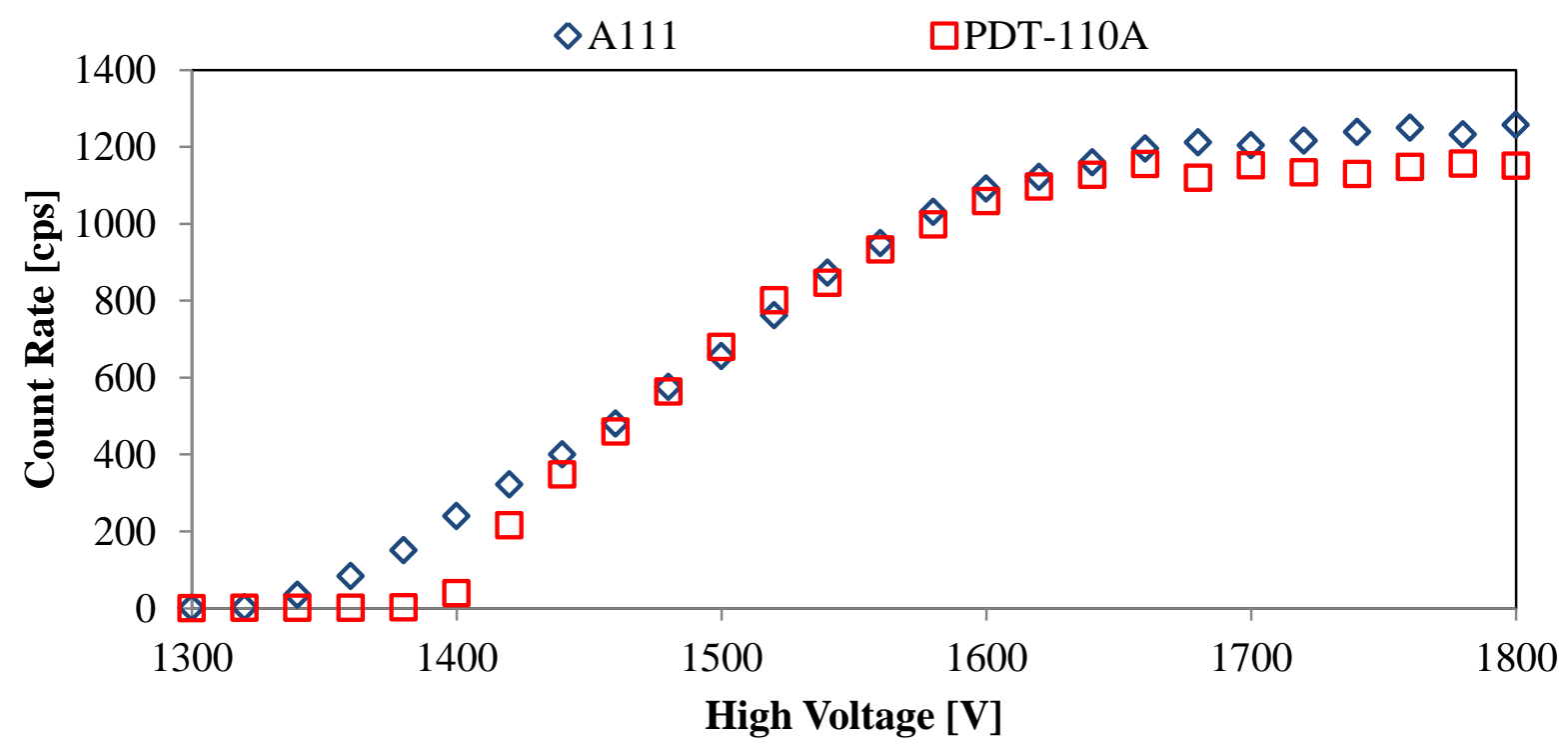

Figure 2-14. High voltage plateaus with ${ }^{252} \mathrm{Cf}$ source alone for A111 and PDT-110A preamplifiers.

The A111 plateau was used as the reference in the radium measurements because it is the preamplifier that is used in DDSI. There was neutron room background that was not negligible; 
however the background was present in both the ${ }^{252} \mathrm{Cf}$ alone and ${ }^{252} \mathrm{Cf}+$ radium measurements, therefore it can be ignored for the purposes of this study.

High voltage plateaus were measured with ${ }^{252} \mathrm{Cf}+$ radium at different distances ranging from 10-70 $\mathrm{cm}$ from the detector pod. Figure 2-15 contains results from the A111 preamplifier, and Figure 2-16 contains results from the PDT-110A preamplifier. $1 \sigma$ error bars are smaller than the markers used and are thus not shown. Each plateau contains both the A111 and PDT 110A preamplifier data, and has the ${ }^{252} \mathrm{Cf}$ alone plateau overlaid for comparison. The region of interest is where the count rates begin to deviate from the reference plateau because that is where the gamma pileup becomes significant. The deviation voltage is indicated in each figure caption and summarized in Table 2-IV. 


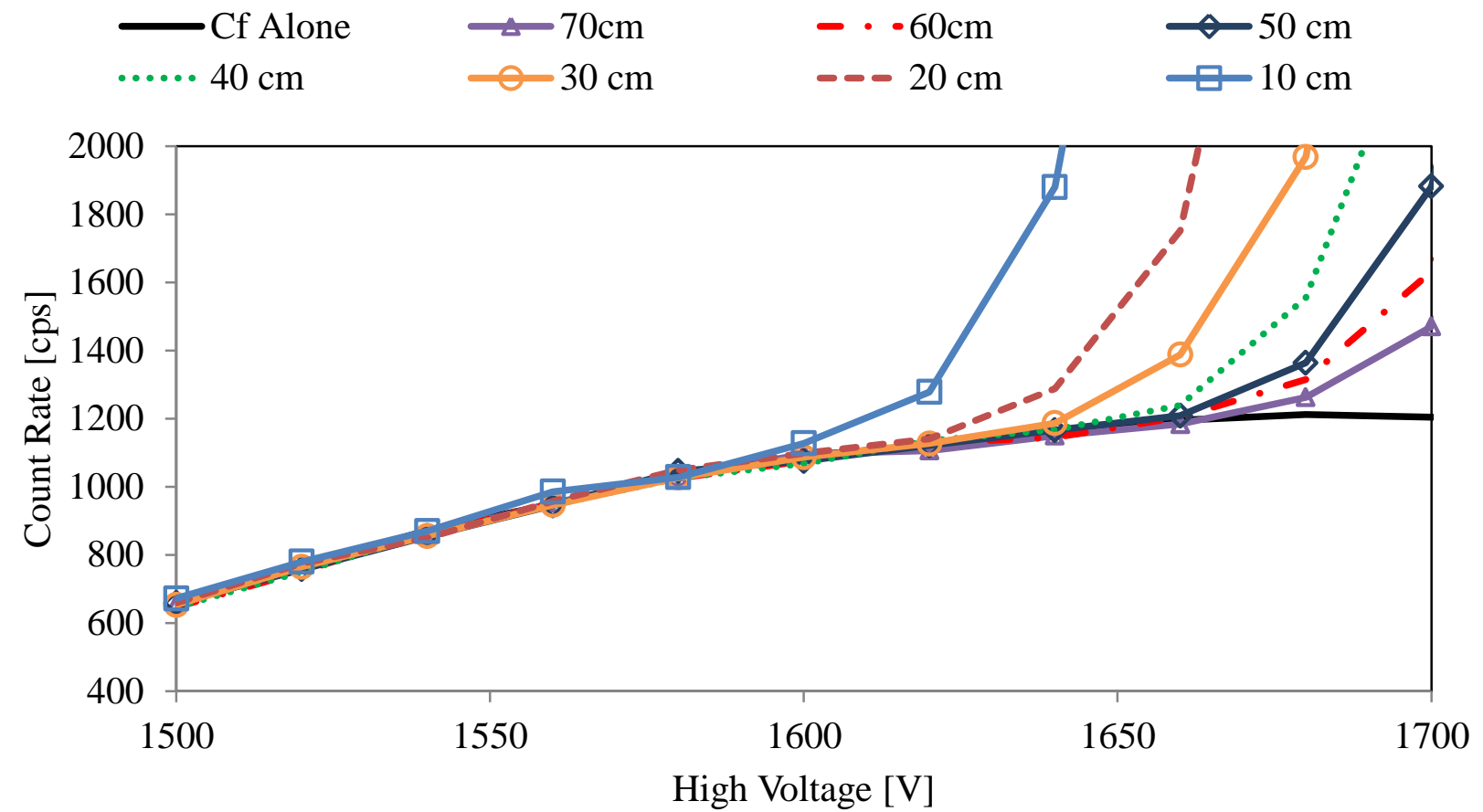

Figure 2-15. High voltage plateaus measured with A111 preamplifier with ${ }^{226}$ Ra source at varying distances. For the design basis of $20 \mathrm{Rad} / \mathrm{hr}$ at $20 \mathrm{~cm}$, the deviation voltage is $1640 \mathrm{~V}$.

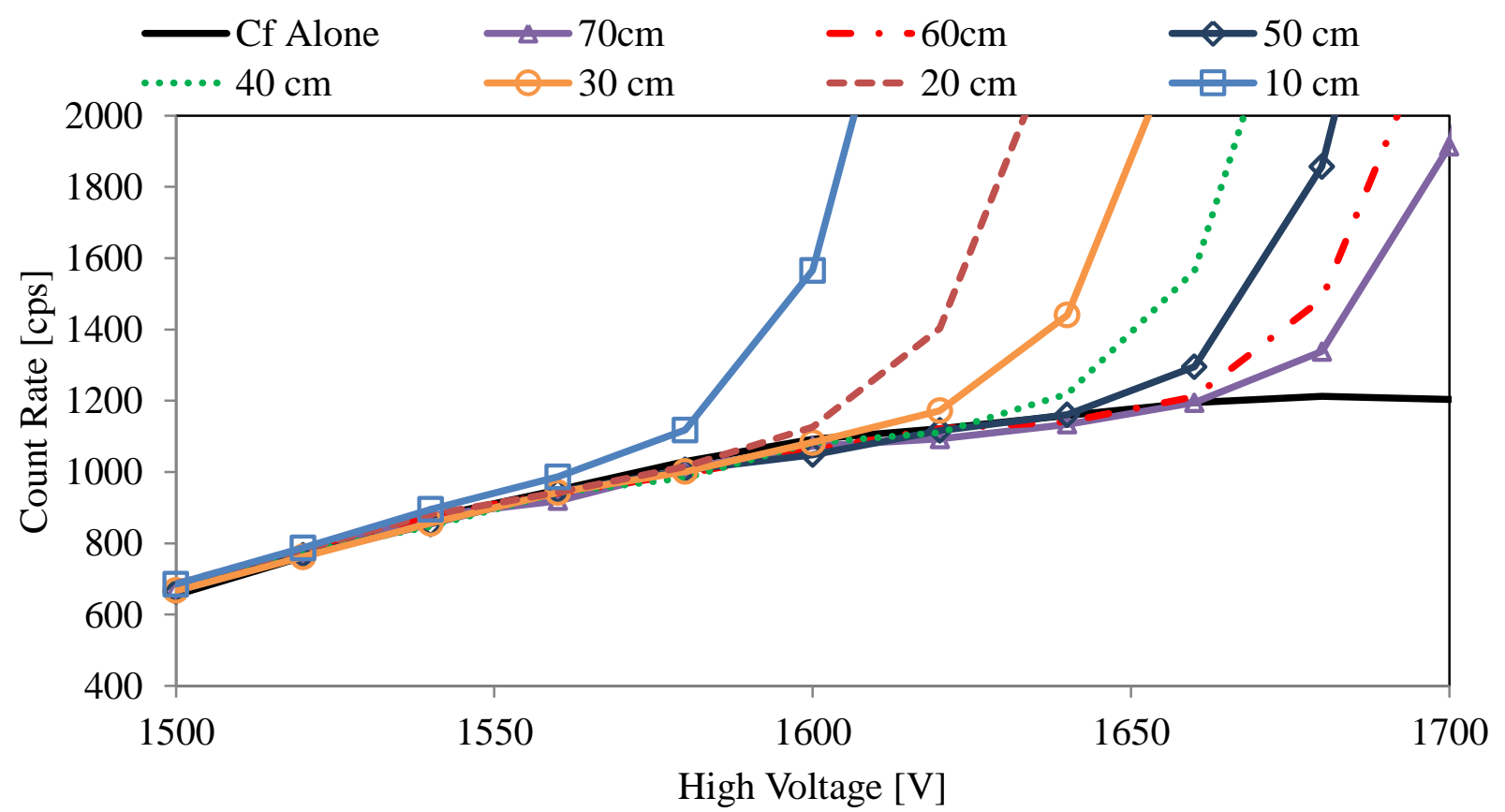

Figure 2-16. High voltage plateaus measured with PDT-110A preamplifier with ${ }^{226}$ Ra source at varying distances. For the design basis of $20 \mathrm{Rad} / \mathrm{hr}$ at $20 \mathrm{~cm}$, the deviation voltage is $1620 \mathrm{~V}$. 
Table 2-IV. Summary of high voltage levels where deviation from ${ }^{252} \mathrm{Cf}$ plateau indicates failing gamma pileup rejection.

\begin{tabular}{cccc}
\hline Distance [cm] & $\begin{array}{c}\text { Approximate Gamma } \\
\text { Dose [R/hr] }\end{array}$ & $\begin{array}{c}\text { Deviation Voltage for } \\
\text { PDT-110A [V] }\end{array}$ & $\begin{array}{c}\text { Deviation Voltage for } \\
\text { A111 [V] }\end{array}$ \\
\hline 10 & 50 & 1580 & 1620 \\
20 & 21 & 1620 & 1640 \\
30 & 9 & 1640 & 1660 \\
40 & 6 & 1660 & 1680 \\
50 & 4.3 & 1660 & 1680 \\
60 & 3.1 & 1680 & 1680 \\
70 & 2.6 & 1680 & 1680 \\
\hline
\end{tabular}

With the design basis of approximately $20 \mathrm{R} / \mathrm{hr}$ ( $20 \mathrm{~cm}$ distance) the count rate began to deviate significantly from the reference plateau at $1640 \mathrm{~V}$ for the A111 preamplifier used in the DDSI instrument. This indicated that the best operating HV for the DDSI setup was $1620 \mathrm{~V}$ in order to accommodate the highest expected dose rate cases of $20 \mathrm{R} / \mathrm{hr}$ without changing the applied voltage. The PDT-110A preamplifier did not perform pileup rejection as efficiently as the A111 preamplifier because it is slower and cannot efficiently prevent pileup.

Also of interest was the effect of gamma dose on the neutron detection efficiency. Figure 2-17 shows the change in count rate as a function of gamma dose for an average of 1600,1620, and $1640 \mathrm{~V}$ measurements. 


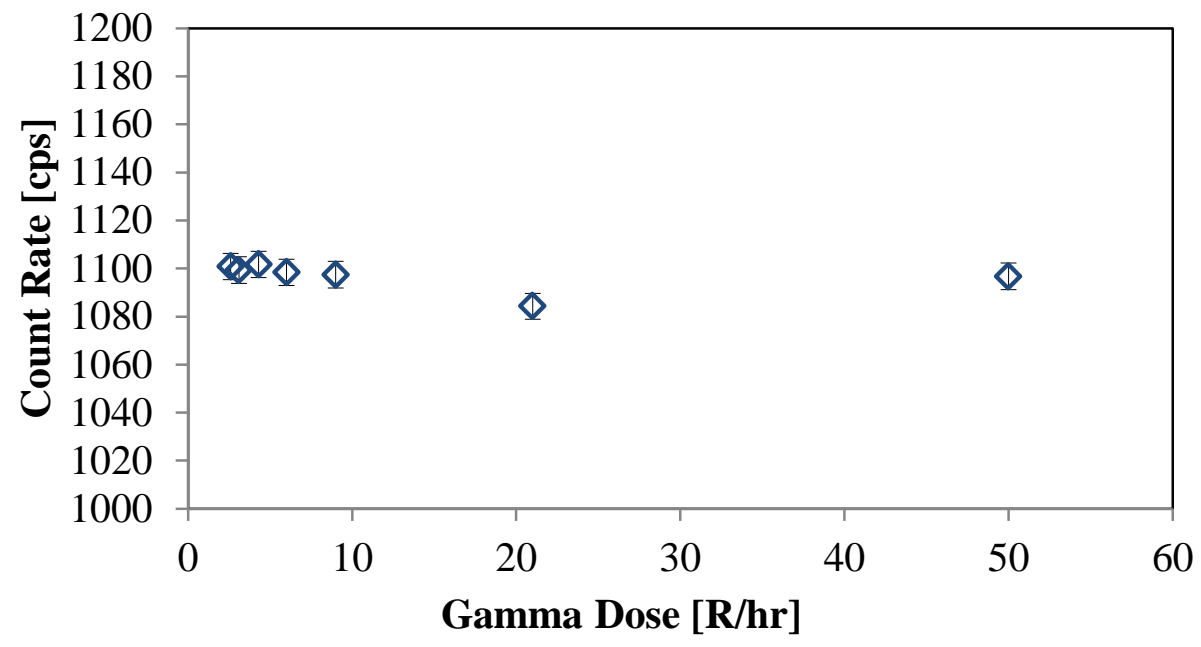

Figure 2-17. Count rate demonstrating neutron detection efficiency at different gamma doses. Efficiency remains constant within statistical uncertainties as the dose increases. $1 \sigma$ uncertainties are shown.

\subsection{Data Acquisition System}

For spent fuel assay it is anticipated that count rates in DDSI will be above 1 million counts per second. The pulse width of the output pulses from the PDT A111 preamplifiers is set by considering the maximum count rate expected from the hottest fuel assembly to be measured. This was estimated based on spent fuel assembly simulations and efficiency calculations to be approximately $6 \cdot 10^{6} \mathrm{cps}$. This restricts the sampling frequency to a maximum of $25 \mathrm{MHz}$. For this data collection mode, raw data streams are written directly into the National Instruments data acquisition (DAQ's) storage disks without being stored in RAM and must be compressed in post-processing. Analog data are converted into binary data: 0 for no pulse at a specified time and 1 for a pulse detected. This produces uniform file sizes for a set sampling frequency, given in Table $2-\mathrm{V}$, regardless of source strength or actual count rate. These data must be compressed and converted to the unsigned 32-bit format in order to be analyzed. The LabVIEW files produced are called technical data management (TDMS) files and they contain information about the times pulses were collected as well as the channel in which the collection took place. Compression and conversion software was written to split the TDMS files into two simple binary files each with a 1-D list of unsigned, 32 bit integers: .tks files to store neutron arrival times and dat files to store channel information [43]. This conversion process takes considerably more time than the data acquisition itself. Table $2-\mathrm{V}$ gives a 
summary of various file sizes and compression times for different sampling frequencies for a DDSI measurement.

Table 2-V. Summary of TDMS file sizes and compression times for $1 \mathrm{hr}$ measurements with different sampling frequencies.

\begin{tabular}{ccccc}
\hline $\begin{array}{c}\text { Sampling } \\
\text { Frequency }\end{array}$ & $\begin{array}{c}\text { TDMS } \\
\text { file size } \\
{[\mathbf{M B}]}\end{array}$ & $\begin{array}{c}\text { Data storage needed } \\
\text { for } \mathbf{1} \text { hr measurement } \\
\text { [GB] }\end{array}$ & $\begin{array}{c}\text { Time to compress 5 s } \\
\text { of measured data [s] }\end{array}$ & $\begin{array}{c}\text { Compression time } \\
\text { / measurement } \\
\text { time }\end{array}$ \\
\hline $5 \mathrm{MHz}$ & $196 \mathrm{MB}$ & $144 \mathrm{~GB}$ & $15.2 \mathrm{~s}$ & 3.04 \\
$10 \mathrm{MHz}$ & $394 \mathrm{MB}$ & $284 \mathrm{~GB}$ & $31.2 \mathrm{~s}$ & 6.24 \\
$15 \mathrm{MHz}$ & $602 \mathrm{MB}$ & $434 \mathrm{~GB}$ & $49.2 \mathrm{~s}$ & 9.84 \\
$20 \mathrm{MHz}$ & $784 \mathrm{MB}$ & $564 \mathrm{~GB}$ & $66.2 \mathrm{~s}$ & 13.24 \\
\hline $25 \mathrm{MHz}$ & $978 \mathrm{MB}$ & $704 \mathrm{~GB}$ & $83.8 \mathrm{~s}$ & 16.76 \\
\hline
\end{tabular}

Given the data presented in Table 2-V, if operating at a $15 \mathrm{MHz}$ sampling frequency, for example, approximately 10 times the measurement time must be allotted for compression. Thus a 1 hour measurement requires 10 hours of compression time. This could present a challenge if many hours of data are to be collected in a single day because leaving the data in the form of TDMS files requires too much space to be practical, therefore compression of the measurement files should start as soon as it is feasible.

To maximize compression and post-processing efficiency, a lower sampling frequency is preferable. To operate at $5 \mathrm{MHz}$, the minimum pulse width is $200 \mathrm{~ns}$. A time-interval distribution collected from a single detector can be analyzed to determine the recovery time of that detector [44]. This was found to be approximately $600 \mathrm{~ns}$ for the DDSI tube and preamplifier combination. Therefore the pulse width can be $250 \mathrm{~ns}$ to ensure detection with a $5 \mathrm{MHz}$ sampling frequency, and no pulses will be lost due to digital pulse overlap because the detector recovery time is longer than the pulse width. It is beneficial to keep the pulse width low to keep deadtime low.

Each entry in the .dat and .tks files is an unsigned 32 bit integer. Assuming a $5 \mathrm{MHz}$ sampling frequency, the measurement itself will therefore require $144 \mathrm{~GB} / \mathrm{hr}$ of storage. A lower count rate will result in smaller compressed files but the same size TDMS files. This allows for approximately 80 hours of measurements on the $24 \mathrm{~TB}$ hard drives available in the DAQ, assuming that the 
measurements are sufficiently spaced to allow for implementation of the compression and conversion software after each measurement. Given the addition of the $20 \mathrm{~TB}$ of external hard drives purchased, data can be transferred from the DAQ to the external drives to possibly allow additional measurements if transfer speeds are fast enough to meet the time constraints of the individual measurement campaign.

\subsection{RAD production and analysis software}

A team at LANL developed the FastTapX software in order to efficiently convert list-mode data to Rossi-alpha distributions. FastTapX was an expansion of VBTap [45], the original multiplicity software developed for an ESARDA benchmark exercise. The original capabilities revolved around multiplicity analysis and a software version of a shift register. It was developed to analyze PTRAC output files from MCNP and the FastTapX expansion extended the capabilities to simulate and analyze various pathologies of experimental data such as deadtime and double pulsing [46]. FastTapX also included Rossi-alpha distribution production from list mode data (simulated or experimental) as well as Feynman-Y [47] and time interval distribution production [48]. For this work only the RAD production and shift register features are utilized.

To produce RADs from DDSI experimental data, FastTapX sorts the detection times after combining all 56 detectors, and then calculates the time between each pulse within the user-defined time window after the trigger. The times are sorted into a histogram using user-defined bin widths.

Analysis of the RADs is conducted using software developed by the author of this thesis written in Python (example Appendix D). RAD data are read from the output files of FastTapX, and a series of fitting algorithms are applied to determine the fast and slow die-away times in more complex fuel, early die-away time, ratio of magnitudes of the fast and slow RAD components, doubles rate in the $24 \mu \mathrm{s}$ (fresh fuel) or $64 \mu \mathrm{s}$ (spent fuel) gate, and any other parameters of interest. A non-linear least squares fitting algorithm is applied to determine the exponential fits. Initial guesses for the die-away time and magnitude of the curve are required for fitting algorithm. Any single exponential RAD

(such as from ${ }^{252} \mathrm{Cf}$ alone in DDSI) has an initial guess of $20 \mu$ s based on the known detector die- 
away time of approximately 18-19 $\mu$ s. An example of a single exponential RAD from a spontaneous fission source alone is given in Figure 2-18.

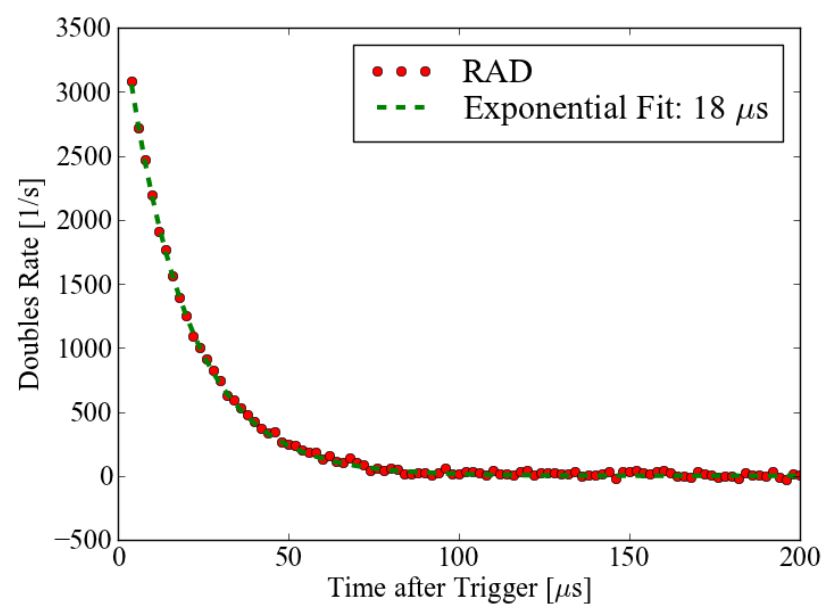

Figure 2-18. Simulated RAD from ${ }^{252} \mathrm{Cf}$ spontaneous fission source in DDSI, which exhibits only a single exponential component.

For DDSI measurements, a double exponential fit is always applied when fissile material is measured for the reasons outlined in detail in Section 1.5. A single exponential fit to such a measurement is not adequate. Any RAD measured from spent fuel is fit in the late time domain first and has an initial guess of $100 \mu \mathrm{s}$. The fast curve fit follows and again has an initial guess of $18 \mu \mathrm{s}$, congruent with that of the detector. The early die-away fit always has an initial guess of $30 \mu \mathrm{s}$. The initial guess for the magnitude of the curve is set as $1 \%$ of the singles rate based on previous results.

The approach for fitting the fast and slow curves of a double exponential RAD such as from fuel was done through several steps.

1) The late time domain was defined as approximately $100 \mu$ s onward for spent fuel assemblies, as this is the time region where thermalized neutrons from later in a fission chain appear in coincidence with the trigger event. The RAD from 100-200 $\mu$ s was fit with a single exponential and extrapolated back to $0 \mu \mathrm{s}$ as shown in Figure 2-19. 


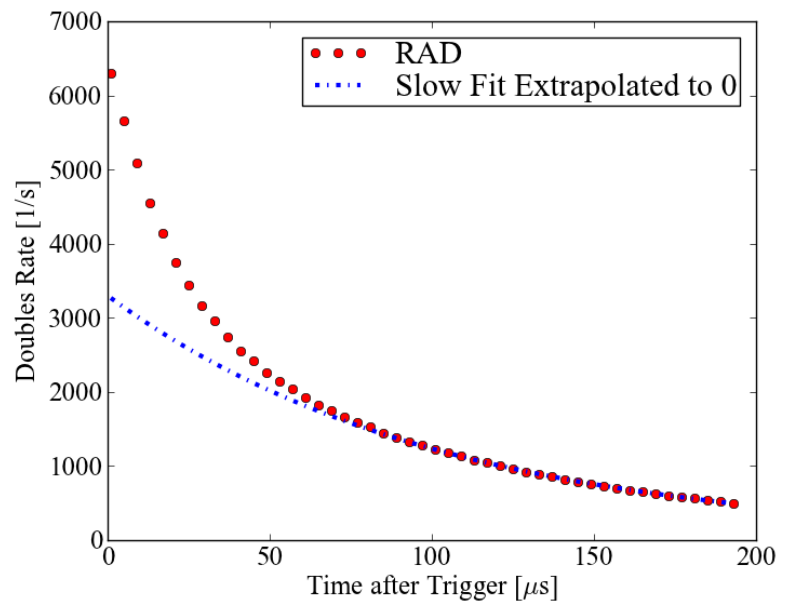

Figure 2-19. RAD with slow component shown. Fit is from 100-200 $\mu$ s and extrapolated back to $0 \mu$ s.

2) Once the slow fit was determined, the value at each point was subtracted from the RAD leaving a single exponential only at the early time domain. The curve dies away to approach 0 in the late region.

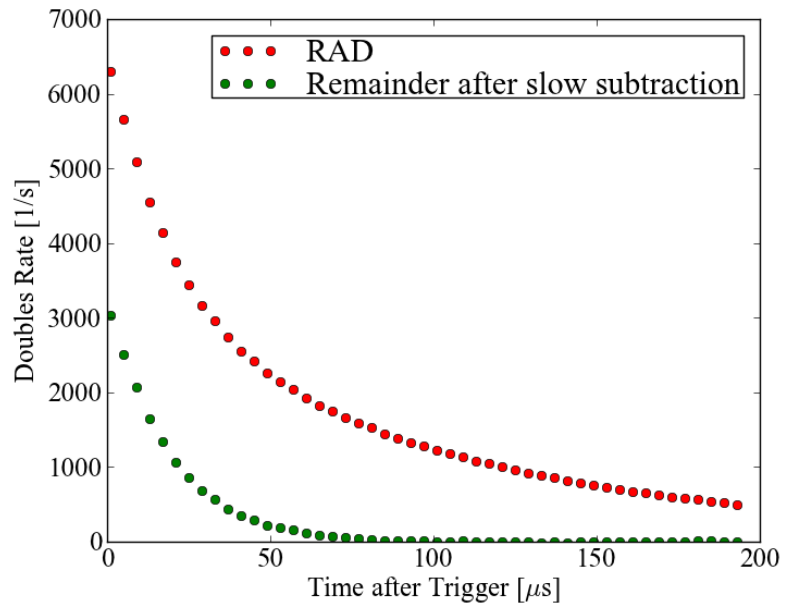

Figure 2-20. RAD (in green) with slow component subtracted leaving another single exponential from which the fast component will come.

3) The fast curve was fit to the remaining values after the slow fit was subtracted. The result is shown in Figure 2-21. 


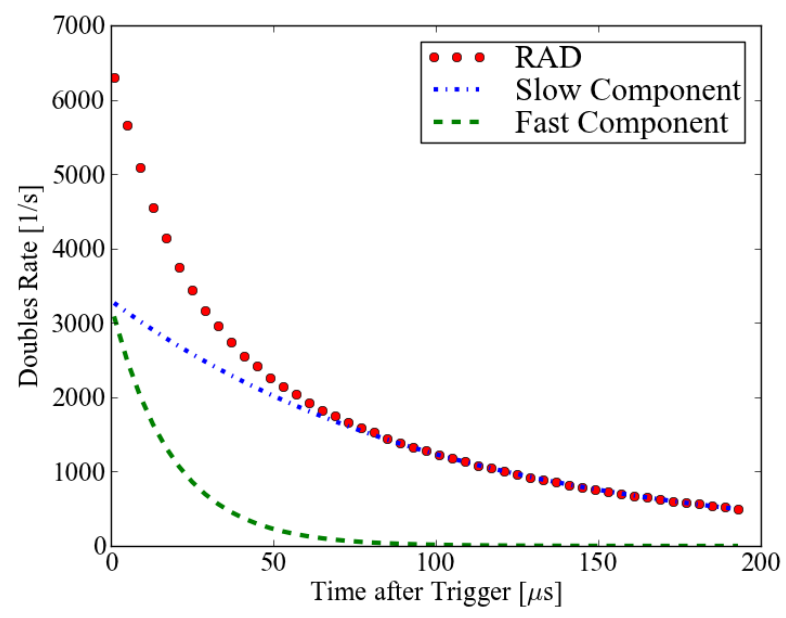

Figure 2-21. RAD with slow and fast fits shown.

The doubles rate can be determined from the RAD by summing the distribution over whichever time interval would have been used as the coincidence gate in a shift register, after accidentals have been subtracted from the distribution. For example, a $24 \mu$ s gate following a $4 \mu$ s predelay was used in fresh fuel measurements with the DDSI instrument (discussed in 0). In this case, the $2 \mu$ s bins from 4-28 would be integrated to determine the double rate. The same procedure is done for the time bins in the MCNP tally output. In principle, this gives statistically the same answer as a shift register operating with a $4 \mu$ s predelay and a $24 \mu$ s gate, assuming the correct value for accidentals has been subtracted. 


\section{Chapter 3. SPENT NuClear Fuel}

In order to understand how best the DDSI system can be used for safeguards and material accountancy, the way spent nuclear fuel is affected by BU, CT, and IE, and other reactor variables must be understood. Commercial pressurized light-water reactor (PWR) fuel was used in the simulations of the DDSI instrument. PWRs are the most common type of nuclear reactor and account for two-thirds of the current worldwide nuclear generating capacity. The PWR utilizes highly pressurized ( 15 MPa) light water as both moderator and primary coolant [49].

The PWR assemblies considered in this DDSI research are Westinghouse type containing 264 fuel rods and 25 control rod channels, arranged in a 17x17 array as shown in Figure 3-1. The fuel rods are comprised of a column of ceramic pellets of uranium dioxide, about $1 \mathrm{~cm}$ in diameter and $2 \mathrm{~cm}$ in length. The column of pellets is clad and sealed in zirconium alloy tubes about $4 \mathrm{~m}$ long. The uranium is enriched to various levels up to $5 \%{ }^{235} \mathrm{U}$.

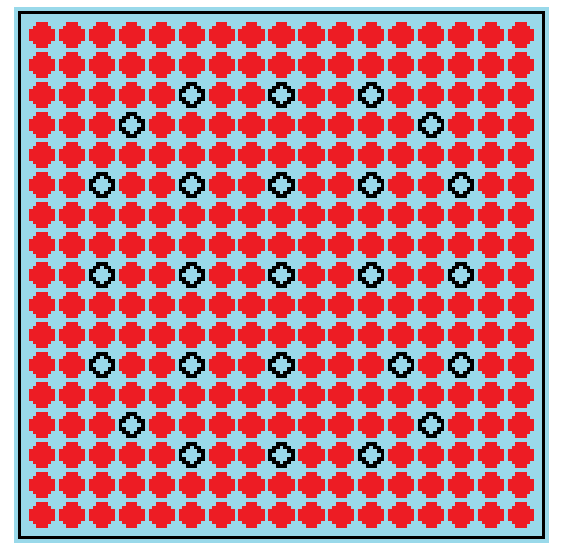

Figure 3-1. Cross-sectional schematic of a PWR fuel assembly used in MCNP simulations with 25 empty channels for control rods and 264 fuel pins. 


\subsection{Isotopic content and changes with reactor variables}

Once an assembly has been used as reactor fuel for a sufficient amount of time and is no longer deemed useful as burnable fuel, it is considered to be spent. Spent fuel is classified as high-level waste, and the total high-level activity from the spent fuel depends on the nature of the reactor fuel cycle. The once-through cycle (no reprocessing of fuel) results in spent fuel with activity that persists for hundreds of thousands of years because of long-lived radioactive isotopes. A closed cycle, where reprocessing occurs, involves returning the plutonium isotopes to the reactor where, upon fissioning, they are mostly transformed into short-lived fission products [49]. The closed cycle, or reprocessing, approach is not supported by all states because of the proliferation threat of separating the plutonium isotopes from the rest of the spent fuel. A nominal 1-GWe (giga watt electric) reactor burns about $1 \mathrm{t}$ of fissile material per year, which results in about $200 \mathrm{~kg}$ of $\mathrm{Pu}$ [50]; this is about 25 Significant Quantities, defined by the IAEA as $8 \mathrm{~kg}$.

The BU, or fuel utilization, is a measure of how much energy is extracted from the fuel source during the burning process. Therefore assemblies that have been in a reactor longer typically have higher BU than those that spend a shorter time in the core. Higher IE typically allows for higher fuel utilization, and similarly, lower IE assemblies are typically not able to be burned as much. BU in the context of this work is expressed in terms of Giga-Watt days produced per metric ton of initial uranium, or $\mathrm{GWd} / \mathrm{tU}$. As fuel is burned, it undergoes transformations in material composition due to fission which splits fissionable isotopes into smaller constituents, and nuclear transmutation through neutron absorption typically followed by $\beta$ decays. Figure 3-2 shows the production processes for major transuranics of interest in spent fuel. 


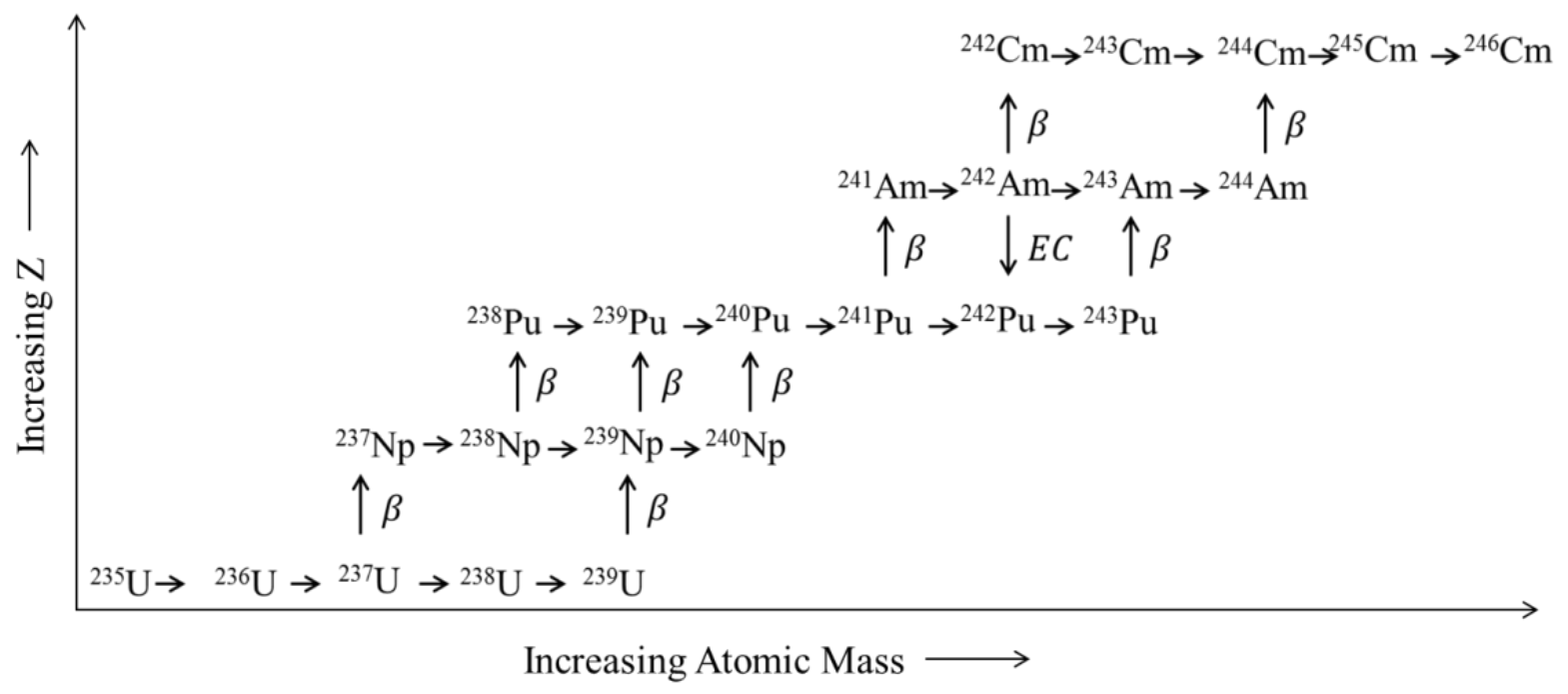

Figure 3-2. Production schematic for high-z transuranics through the fuel burning process. Neutron absorption processes are depicted by horizontal arrows.

Though uranium is the heaviest isotope in fresh, low-enriched uranium fuel, the burning process produces many heavier, long-lived isotopes with unique properties that help to characterize the spent fuel. The more an assembly is burned, the more opportunity there is for multiple neutron capture and other processes that produce the transuranic isotopes. Higher burned assemblies will have generally higher concentrations of these transuranic isotopes [51]; however, a higher IE assembly will have a lower concentration of the higher transuranic isotopes relative to a lower IE assembly with the same $\mathrm{BU}$. The reasoning for this becomes clearer when considering the transuranic production processes in Figure 3-2. The isotope ${ }^{238} \mathrm{U}$ produces transuranics through a series of neutron absorption followed by beta decays which are all energetically favored. However, ${ }^{235} \mathrm{U}$ has a more difficult path to produce these transuranics because of the need for double neutron absorptions before beta decay to ${ }^{237} \mathrm{~Np}$ coupled with the much larger fission cross section relative to radiative capture cross section for thermal neutrons. Figure 3-3 shows the change in mass as a function of BU and IE for ${ }^{240} \mathrm{Pu}$ and ${ }^{244} \mathrm{Cm}$, both important isotopes for the DDSI signal. The results come from simulated spent fuel which will be discussed in detail in the next Section. 


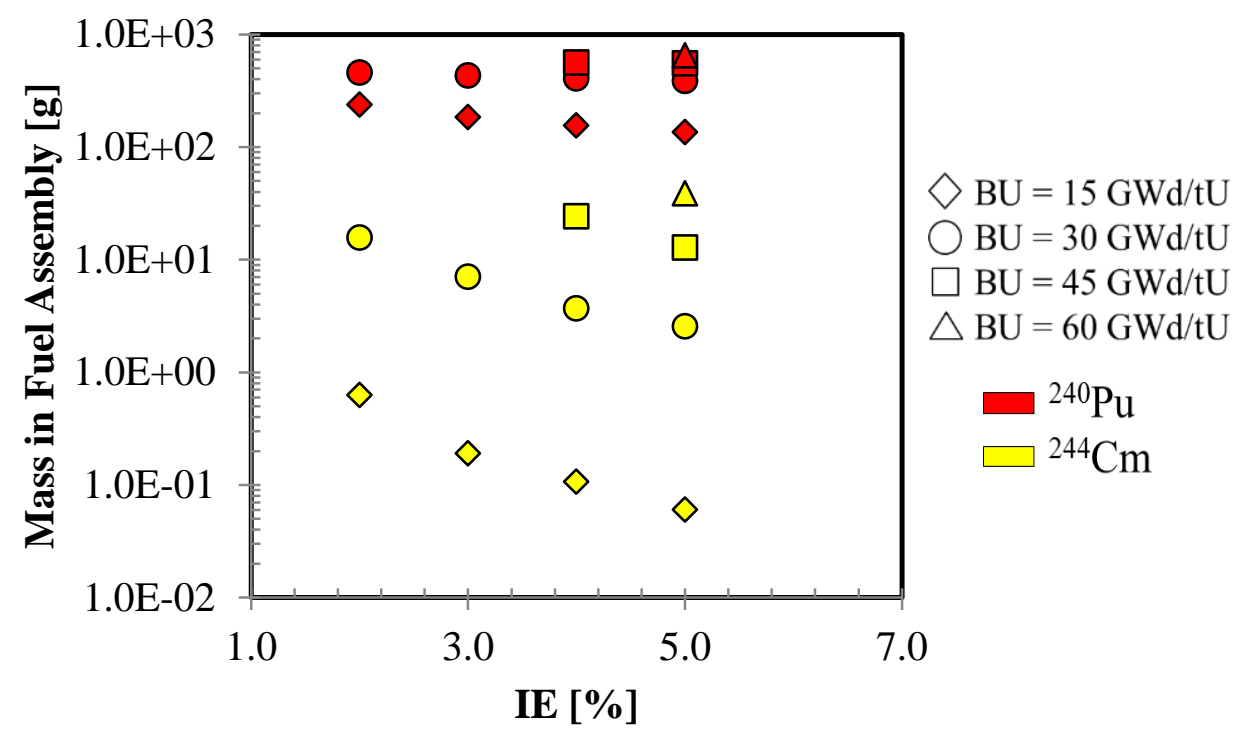

Figure 3-3. Mass of ${ }^{240} \mathrm{Pu}$ and ${ }^{244} \mathrm{Cm}$ in simulated $5 \mathrm{y}$ cooled fuel assemblies as a function of $\mathrm{BU}$ and IE. Mass increases with decreasing IE and increases with increasing BU.

As burnup increases, both ${ }^{240} \mathrm{Pu}$ and ${ }^{244} \mathrm{Cm}$ masses increase. As initial enrichment increases, however, the masses of these isotopes decrease because of the production methods described in the previous paragraph.

\subsection{Simulating Spent Fuel with MCNP}

Spent fuel is a challenging material to simulate because of the wide variety of fissile, fertile, and spontaneous fission isotopes, as well as hundreds of different fission products and neutron absorbers. Simulation of the fuel burning process requires significant computing power and even so, many approximations must be made. For example, unless exact operating conditions are known to accurately model SF assembly shuffling in the reactor core, infinitely reflected boundary conditions (i.e. assuming no shuffling scheme) are used when simulating burnup which has been shown to result in 5-10\% errors in plutonium composition [52].

A series of high-fidelity simulations were conducted through the NGSI project to develop spent fuel libraries containing realistic simulated spent fuel assemblies with accurate material compositions [53,54]. To construct these libraries, a representative MCNP [55] model of a portion of a hypothetical reactor core is created. Monteburns is then used to perform burnup calculations by 
solving the multi-group diffusion equations at time $=0$, then using the fluxes from time $=0$ to compute those at time $=1$, etc [56]. Cinder90 then takes the input files from Monteburns and performs subsequent decay calculations for specific cooling times [57]. The decayed compositions are then compiled and converted into material compositions in MCNP input files. A description of all libraries used in this work follows [53]:

a. SFL1: First set of assemblies created by NGSI. Assemblies had infinitely reflected boundaries rather than undergoing shuffling. 17x17 PWR-type assemblies were modeled with MCNP Burnup to simulate burning. Developed at Los Alamos National Laboratory.

b. SFL2a: Considered the "base" library. More realistic assemblies created as a follow-up to SFL1. Half of the pins were modeled then the results were reflected across symmetrically at the end of the modeling. The half-assemblies were shuffled through a full model of $1 / 8$ of a reactor core following traditional core loading practices. Monteburns was used to simulate burnup, and ENDF70 was used for data. Developed at LANL.

c. SFL3: Created as a sensitivity study for operating parameters. The following are varied: control rods, burnable poisons, moderator density and temperature, boron concentration, and fuel temperature. Modeled with SCALE/Triton capability at ORNL.

d. SFL4: "Mystery" assemblies created to test experimental NDA analysis methods developed on other assemblies with unknown parameters. Large variety of parameters varied for this library; described in detail in Appendix B.

e. SFL6: Assemblies with missing pins. 40 pins replaced in each assembly with steel or natural uranium. Pins were removed either uniformly or all from the center. Complete assemblies before pin diversions are based on SFL2a assemblies.

All spent fuel libraries were developed with validated and verified codes, however it is impossible to perfectly simulate the production of spent fuel because of the large number of variables involved in power production. The goal of the NGSI project was to create an ensemble of synthetic spent fuel assemblies that span across the full dynamic range of real world SFAs.

\subsubsection{Simulating the Spontaneous Fission Source in Spent Fuel}

The isotopic content of the fuel pins is defined by the spent fuel libraries as outlined in the previous section. However material definitions alone do not serve as a source in MCNP [58] and one must be 
explicitly defined so that MCNP has a method of determining how particles are born in the system. In order to simulate a spontaneous fission source, the fuel pins are first given weighting factors according to the relative amount of spontaneous fission events that would take place in the material in that pin. MCNP then performs random sampling from the distribution of weighting factors to determine which pin each spontaneous fission event will originate in. Once a pin has been determined, the SDEF (source definition) command "PAR=SF" determines the remaining variables for the spontaneous fission event. First, the spontaneous fission isotope is determined proportionately to the product of the atom fraction and spontaneous fission yield for all isotopes in the material definition of the chosen cell [58]. The multiplicity (number of neutrons released in the fission event) is determined based upon the multiplicity distribution of the selected isotope. Finally, the energy of the emitted neutrons is sampled from the Watt spectrum with an average corresponding to that of the selected isotope. The direction and starting location of the particles within the cell is sampled according to the standard Monte-Carlo procedure, though all particles for a particular SF event begin at the same location. It has been shown that in real fission events the direction and energy with which the neutrons are released is not isotropic or uncorrelated [59]. However because of the thermalization that almost always takes place before an emitted neutron will induce fission or be captured in a detector and the likely other larger sources of systematic uncertainties, the added challenge in accessing specialized data libraries and computation time would not be worth the very slight gain in physical correctness.

MCNP has data for 18 spontaneous fission nuclides available for sampling: ${ }^{232} \mathrm{Th},{ }^{232} \mathrm{U},{ }^{233} \mathrm{U},{ }^{234} \mathrm{U}$, ${ }^{235} \mathrm{U},{ }^{236} \mathrm{U},{ }^{238} \mathrm{U},{ }^{237} \mathrm{~Np},{ }^{238} \mathrm{Pu},{ }^{239} \mathrm{Pu},{ }^{240} \mathrm{Pu},{ }^{241} \mathrm{Pu},{ }^{242} \mathrm{Pu},{ }^{241} \mathrm{Am},{ }^{242} \mathrm{Cm},{ }^{244} \mathrm{Cm},{ }^{249} \mathrm{Bk}$, and ${ }^{252} \mathrm{Cf}$. All 18 isotopes are present in spent fuel and are included in the source isotope sampling process. Additional isotopes have been added manually for DDSI simulations in order to incorporate ${ }^{236} \mathrm{Pu},{ }^{246} \mathrm{Cm}$, ${ }^{248} \mathrm{Cm}$, and ${ }^{250} \mathrm{Cf}$. These last four isotopes are not major contributors in most assemblies (<1\% each) but in certain cases, ${ }^{246} \mathrm{Cm}$, for example, contributes on the order of a million fissions/s, which is on the order of a percent and therefore cannot be neglected. The currently accepted spontaneous fission parameters for each isotope are incorporated into the model.

The complete list of SF isotopes included in the simulated assemblies along with half-lives, SF branching percentages, masses in a $45 \mathrm{GWd} / \mathrm{tU}$ BU, 5 y CT, 4\% IE assembly, and fission rates are shown in Table 3-I. 
Table 3-I. Spontaneous fission isotopes (written in the format "ZZAAA" where $\mathrm{ZZ}$ is the atomic number and $\mathrm{AAA}$ is the mass number) and their contribution to the total fission rate in a 45 GWd/tU BU, 4\% IE, 5 y CT assembly.

\begin{tabular}{|c|c|c|c|c|c|}
\hline Isotope & $\mathbf{t}_{1 / 2}[\mathbf{y}]$ & $\mathrm{SF}[\%]$ & Mass [g] & SF Rate $\left[\mathbf{s}^{-1}\right]$ & $\begin{array}{l}\text { Fractional } \\
\text { Contribution }\end{array}$ \\
\hline 90230 & $7.54 \mathrm{E}+04$ & $3.80 \mathrm{E}-12$ & $1.48 \mathrm{E}-03$ & 4.30E-08 & $4.15 \mathrm{E}-16$ \\
\hline 90232 & $1.41 \mathrm{E}+10$ & $1.80 \mathrm{E}-09$ & $5.94 \mathrm{E}-04$ & $4.34 \mathrm{E}-11$ & 4.19E-19 \\
\hline 91231 & $3.28 \mathrm{E}+04$ & $1.60 \mathrm{E}-11$ & $1.58 \mathrm{E}-04$ & $4.42 \mathrm{E}-08$ & $4.28 \mathrm{E}-16$ \\
\hline 92233 & $1.59 \mathrm{E}+05$ & $6.00 \mathrm{E}-11$ & $1.94 \mathrm{E}-03$ & $4.16 \mathrm{E}-07$ & $4.02 \mathrm{E}-15$ \\
\hline 92234 & $2.46 \mathrm{E}+05$ & $1.64 \mathrm{E}-09$ & $6.93 \mathrm{E}+01$ & $2.62 \mathrm{E}-01$ & $2.53 \mathrm{E}-09$ \\
\hline 92235 & $7.04 \mathrm{E}+08$ & 7.00E-09 & $3.84 \mathrm{E}+03$ & $2.15 \mathrm{E}-02$ & $2.08 \mathrm{E}-10$ \\
\hline 92236 & $2.34 \mathrm{E}+07$ & $9.40 \mathrm{E}-08$ & $2.50 \mathrm{E}+03$ & $5.63 \mathrm{E}+00$ & $5.44 \mathrm{E}-08$ \\
\hline 92238 & 4.47E+09 & $5.45 \mathrm{E}-05$ & $4.36 \mathrm{E}+05$ & $2.96 \mathrm{E}+03$ & $2.86 \mathrm{E}-05$ \\
\hline 93237 & $2.14 \mathrm{E}+06$ & $2.00 \mathrm{E}-10$ & $2.84 \mathrm{E}+02$ & $1.48 \mathrm{E}-02$ & $1.43 \mathrm{E}-10$ \\
\hline 94236 & $2.86 \mathrm{E}+00$ & $1.37 \mathrm{E}-07$ & 4.99E-05 & $1.34 \mathrm{E}+00$ & $1.30 \mathrm{E}-08$ \\
\hline 94238 & $8.77 \mathrm{E}+01$ & $1.85 \mathrm{E}-07$ & $1.13 \mathrm{E}+02$ & $1.32 \mathrm{E}+05$ & $1.28 \mathrm{E}-03$ \\
\hline 94239 & $2.41 \mathrm{E}+04$ & $3.00 \mathrm{E}-10$ & $2.58 \mathrm{E}+03$ & $1.78 \mathrm{E}+01$ & $1.72 \mathrm{E}-07$ \\
\hline 94240 & $6.56 \mathrm{E}+03$ & $5.75 \mathrm{E}-06$ & $1.28 \mathrm{E}+03$ & $6.20 \mathrm{E}+05$ & $5.99 \mathrm{E}-03$ \\
\hline 94241 & $1.44 \mathrm{E}+01$ & $2.40 \mathrm{E}-14$ & $5.63 \mathrm{E}+02$ & 5.17E-01 & $5.00 \mathrm{E}-09$ \\
\hline 94242 & $3.73 \mathrm{E}+05$ & $5.54 \mathrm{E}-04$ & $3.54 \mathrm{E}+02$ & $2.87 \mathrm{E}+05$ & $2.78 \mathrm{E}-03$ \\
\hline 94244 & $8.08 \mathrm{E}+07$ & $1.21 \mathrm{E}-01$ & $2.50 \mathrm{E}-02$ & $2.03 \mathrm{E}+01$ & $1.97 \mathrm{E}-07$ \\
\hline 95241 & $4.32 \mathrm{E}+02$ & 4.30E-10 & $1.95 \mathrm{E}+02$ & $1.06 \mathrm{E}+02$ & $1.03 \mathrm{E}-06$ \\
\hline 95243 & $7.37 \mathrm{E}+03$ & 3.70E-09 & $8.31 \mathrm{E}+01$ & $2.27 \mathrm{E}+01$ & $2.20 \mathrm{E}-07$ \\
\hline 96242 & $4.46 \mathrm{E}-01$ & $6.37 \mathrm{E}-06$ & $3.21 \mathrm{E}-03$ & $2.51 \mathrm{E}+04$ & $2.42 \mathrm{E}-04$ \\
\hline 96243 & $2.91 \mathrm{E}+01$ & 5.30E-09 & $1.62 \mathrm{E}-01$ & $1.60 \mathrm{E}+01$ & $1.55 \mathrm{E}-07$ \\
\hline 96244 & $1.81 \mathrm{E}+01$ & $1.37 \mathrm{E}-04$ & $2.48 \mathrm{E}+01$ & $1.02 \mathrm{E}+08$ & $9.83 \mathrm{E}-01$ \\
\hline 96245 & $8.50 \mathrm{E}+03$ & $6.10 \mathrm{E}-07$ & $2.01 \mathrm{E}+00$ & $7.79 \mathrm{E}+01$ & $7.53 \mathrm{E}-07$ \\
\hline 96246 & $4.73 \mathrm{E}+03$ & $2.63 \mathrm{E}-02$ & $2.24 \mathrm{E}-01$ & $6.71 \mathrm{E}+05$ & $6.48 \mathrm{E}-03$ \\
\hline 96248 & $3.40 \mathrm{E}+05$ & $8.39 \mathrm{E}+00$ & $1.81 \mathrm{E}-04$ & $2.38 \mathrm{E}+03$ & $2.30 \mathrm{E}-05$ \\
\hline 97249 & 8.77E-01 & 4.76E-08 & 3.01E-08 & 8.70E-04 & $8.41 \mathrm{E}-12$ \\
\hline 98249 & $3.51 \mathrm{E}+02$ & 4.40E-07 & $2.37 \mathrm{E}-06$ & $1.58 \mathrm{E}-03$ & $1.53 \mathrm{E}-11$ \\
\hline 98250 & $1.31 \mathrm{E}+01$ & $7.70 \mathrm{E}-02$ & $4.61 \mathrm{E}-07$ & $1.44 \mathrm{E}+03$ & $1.39 \mathrm{E}-05$ \\
\hline 98252 & $2.65 \mathrm{E}+00$ & $3.09 \mathrm{E}+00$ & $4.20 \mathrm{E}-08$ & $2.58 \mathrm{E}+04$ & $2.49 \mathrm{E}-04$ \\
\hline Total & & & & $1.03 \mathrm{E}+08$ & $1.00 \mathrm{E}+00$ \\
\hline
\end{tabular}

The boxed rows indicate the isotopes which are missing from the standard MCNP data tables and have been left out of the source term because of their negligible total spontaneous fission rate. Other 
SF isotopes that were not in MCNP and had a non-negligible contribution were added by hand using the FMULT (fission multiplier) card.

\subsection{Neutron Production Methods in Spent Fuel and Relative Prominences}

There are several different methods of neutron production occurring in spent fuel including spontaneous fission, induced fission, $(\alpha, n)$ reaction, photo-fission, and (n, $x n)$ reaction. The primary contributor to detected neutrons in most cases is induced fission, and the primary source of neutrons within an assembly is almost always spontaneous fission. This is because each source neutron may go on to produce many more induced fission neutrons. In long cooled fuel $(\alpha, n)$ may take over as the dominant source neutron mechanism because of the relatively short half-lives of Curium isotopes which are the dominant spontaneous fission isotopes in spent fuel.

\subsubsection{Spontaneous Fission}

The primary contributor of spontaneous fission (SF) neutrons in the majority of spent fuel assemblies is ${ }^{244} \mathrm{Cm}$. It has a short half-life of 18.1 years compared to the lifetime of the fuel and relatively high branching ratio for spontaneous fission resulting in a large specific activity of $4.11 \times 10^{6} \mathrm{SF}$ events/g.s. The neutron energy distribution from ${ }^{244} \mathrm{Cm}$ is in the form of a Watt spectrum as shown in Figure 3-4. At very short cooling times, ${ }^{242} \mathrm{Cm}$ dominates the SF output because of its shorter half-life of 0.45 years and specific activity of $7.81 \cdot 10^{6} \mathrm{SF}$ events $/ \mathrm{g} \cdot \mathrm{s}$. After the Curium isotopes, ${ }^{240} \mathrm{Pu}$ is the next most prominent SF contributor with a half-life of 6,563 years and specific activity of $4.83 \cdot 10^{2} \mathrm{SF}$ events/g.s. This isotope will dominate the SF signal at very long cooling times (approximately 60 or more years) because the $\mathrm{Cm}$ isotopes will have mostly decayed away. 


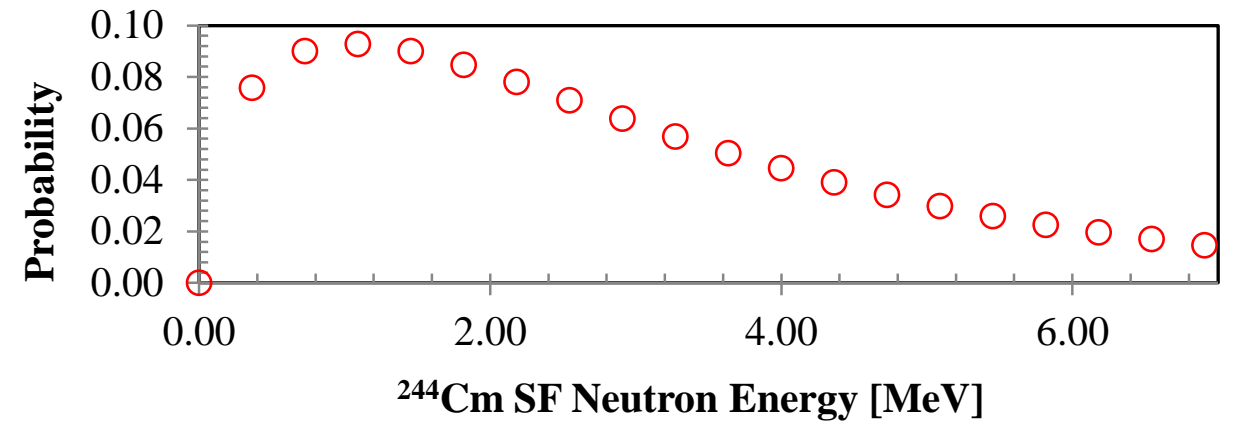

Figure 3-4. Emitted neutron energy distribution from ${ }^{244} \mathrm{Cm}$ spontaneous fission source in SFL2a simulated 45 GWd/tU BU, $4 \%$ IE, 5 y CT assembly.

\subsubsection{Induced Fission}

The prominence of long fission chains in spent fuel results in induced fission being the primary contributor of neutrons to the measured signal. The fissile isotopes: ${ }^{235} \mathrm{U},{ }^{239} \mathrm{Pu}$, and ${ }^{241} \mathrm{Pu}$ are the most prominent isotopes on which induced fission occurs, and their relative contributions depend on the fuel composition, and thus, IE, BU, and CT. The three isotopes have different induced fission moments, shown in Table 3-II, resulting in a different number of neutrons released from each fission on average [60].

Table 3-II. Induced fission moments for ${ }^{235} \mathrm{U},{ }^{239} \mathrm{Pu}$, and ${ }^{241} \mathrm{Pu}$.

\begin{tabular}{cccc}
\hline Moment & ${ }^{235} \mathbf{U}$ & ${ }^{239} \mathbf{P u}$ & ${ }^{241} \mathbf{P u}$ \\
\hline$\overline{\boldsymbol{v}}$ & 2.406 & 2.879 & 2.929 \\
$\overline{\boldsymbol{v}(\boldsymbol{v}-\mathbf{1})}$ & 4.626 & 6.773 & 7.017 \\
$\overline{\boldsymbol{v}(\boldsymbol{v}-\mathbf{1})(\boldsymbol{v}-\mathbf{2})}$ & 6.862 & 12.630 & \\
\hline
\end{tabular}

For spent fuel simulation purposes, only prompt neutrons from induced fission are considered because the number of delayed neutrons is typically only about $1 \%$ of prompt neutrons. The time at which neutrons from each isotope are detected following the original source spontaneous fission can be observed with PTRAC, the particle tracking feature of MCNP [61]. A PTRAC record contains the event history of all the neutrons created from an initial spontaneous fission event including the event histories of any induced fission neutrons created and their subsequent progeny, if any. A specialized PTRAC tally created specifically for the NGSI-SF program records only those histories that have a 
capture event in a user defined cell such as a ${ }^{3} \mathrm{He}$ detector tube of DDSI. Table 3-III shows the isotopes most commonly contributing induced fission neutrons, along with their probability of occurrence, for selected combinations of BU/CT/IE in spent fuel library 1 as determined by PTRAC. Fission percentages given are the percentage of all fission events (spontaneous and induced) contributed by that isotope.

Table 3-III. Total fission contributions of fissioning isotopes (Fis) along with atom fractions (AF) for selected assemblies that demonstrate a variety in SF isotope contributions. The fission percentages do not add up to $100 \%$ because the remainder of the contributions comes from spontaneous fission.

\begin{tabular}{|c|c|c|c|c|c|c|c|c|}
\hline \multirow[t]{2}{*}{ Isotope } & \multicolumn{2}{|c|}{$\begin{array}{l}\text { (1) } \mathrm{BU}=15 \mathrm{IE}=2 \\
\mathrm{CT}=80\end{array}$} & \multicolumn{2}{|c|}{$\begin{array}{l}\text { (2) } \mathrm{BU}=15 \mathrm{IE}=3 \\
\mathrm{CT}=40\end{array}$} & \multicolumn{2}{|c|}{$\begin{array}{l}\text { (3) } \mathrm{BU}=15 \mathrm{IE}=5 \\
\mathrm{CT}=80\end{array}$} & \multicolumn{2}{|c|}{$\begin{array}{l}\text { (4) } \mathrm{BU}=30 \mathrm{IE}=2 \\
\mathrm{CT}=5\end{array}$} \\
\hline & $A F$ & Fis (\%) & $A F$ & Fis (\%) & $A F$ & Fis (\%) & $A F$ & Fis (\%) \\
\hline U-235 & 0.0029 & 29.23 & 0.0055 & 42.50 & 0.0116 & 60.51 & 0.0012 & 12.61 \\
\hline U-238 & 0.321 & 5.00 & 0.3182 & 4.35 & 0.3122 & 4.15 & 0.3149 & 4.58 \\
\hline Pu-239 & 0.0014 & 25.77 & 0.0014 & 22.75 & 0.0014 & 16.37 & 0.0015 & 28.37 \\
\hline Pu-241 & $4.4 \mathrm{e}-6$ & 0.07 & $2.4 \mathrm{e}-5$ & 0.40 & $2.2 \mathrm{e}-6$ & 0.07 & 0.0003 & 7.56 \\
\hline \multirow[t]{2}{*}{ Isotope } & \multicolumn{2}{|c|}{$\begin{array}{l}\text { (5) } \mathrm{BU}=30 \text { IE }=3 \\
\mathrm{CT}=20\end{array}$} & \multicolumn{2}{|c|}{$\begin{array}{l}\text { (6) } \mathrm{BU}=45 \mathrm{IE}=4 \\
\mathrm{CT}=20\end{array}$} & \multicolumn{2}{|c|}{$\begin{array}{l}\text { (7) } \mathrm{BU}=45 \text { IE }=5 \\
\mathrm{CT}=5\end{array}$} & \multicolumn{2}{|c|}{$\begin{array}{l}(8) \mathrm{BU}=60 \mathrm{IE}=5 \\
\mathrm{CT}=5\end{array}$} \\
\hline & $A F$ & Fis (\%) & $A F$ & Fis (\%) & $A F$ & Fis (\%) & $A F$ & Fis (\%) \\
\hline U-235 & 0.0029 & 24.22 & 0.0027 & 20.80 & 0.0048 & 31.58 & 0.0027 & 18.69 \\
\hline U-238 & 0.3129 & 5.04 & 0.3050 & 4.27 & 0.3031 & 4.30 & 0.2982 & 4.85 \\
\hline Pu-239 & 0.0016 & 27.57 & 0.0018 & 27.37 & 0.0020 & 25.24 & 0.0019 & 26.20 \\
\hline Pu-241 & 0.0001 & 2.55 & 0.0002 & 3.52 & 0.0004 & 5.7 & 0.0005 & 7.64 \\
\hline
\end{tabular}

\subsection{3. $(\alpha, n)$ Neutrons}

When alpha particles are emitted in spent fuel, the relatively large concentration of low-Z isotopes in the fuel can result in a high quantity of $(\alpha, n)$ reactions. In most heavy isotopes, $\alpha$-decay is far more probable than spontaneous fission. However the $\alpha$ particles are unable to travel far $(\sim 0.006 \mathrm{~cm})$ in the high density fuel, so will only be absorbed by a low-Z material if in the direct vicinity of the particle release. These $(\alpha, n)$ neutrons can be comparable in number to spontaneous fission neutrons in spent fuel if isotopes with high alpha decay rates such as ${ }^{233} \mathrm{U},{ }^{234} \mathrm{U},{ }^{238} \mathrm{Pu}$ or ${ }^{241} \mathrm{Am}$ are present 
and the fuel has been cooled for approximately 60 years or more [1]. Alpha particles are emitted in spent fuel with an energy spectrum similar to that seen in Figure 3-5, produced from an MCNP simulation of the $(\alpha, n)$ component of spent fuel.

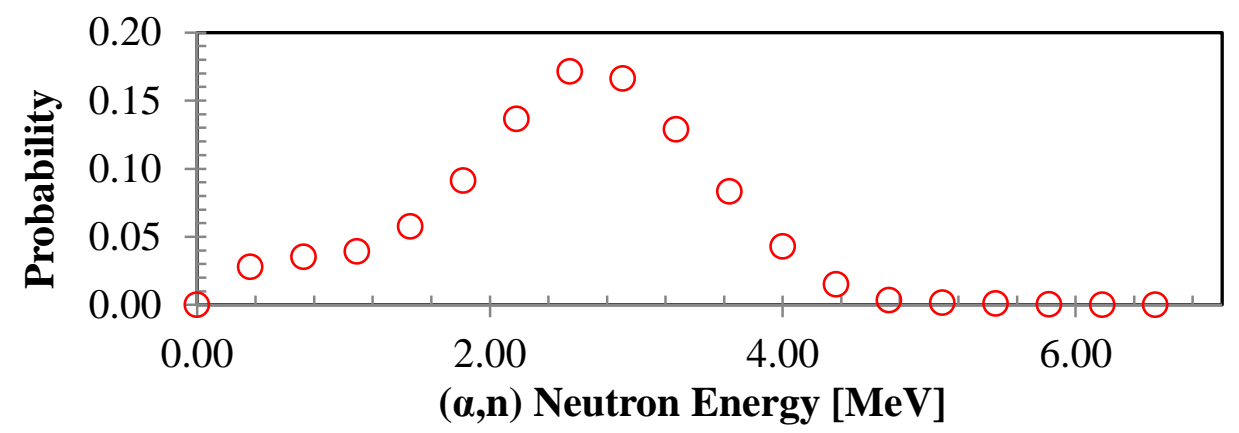

Figure 3-5. Simulated emitted neutron energy distribution from a typical $(\alpha, n)$ source in spent fuel.

Neutrons from $(\alpha, n)$ reactions can be a significant part of the source term in spent fuel and must therefore be accounted for any time the total neutron count rate is considered (i.e., they must be modeled or added to the source term in post-processing or the singles rate cannot be considered accurate). This is important in DDSI measurement analysis when considering singles rates such as the total plutonium determination method discussed in Section 5.2. However in the early die-away method discussed in Section 5.1 and similar ratio-based methods, the singles rate cancels out and the $(\alpha, n)$ term does not need to be accounted for.

\subsubsection{Photo-Fission Neutrons}

Photo-fission can occur in any element if the energy of the incoming gamma-ray is sufficiently high. However the majority of gamma rays emitted in spent fuel are below the typical threshold of approximately $8 \mathrm{MeV}$, resulting in very little photo-fission [1]. Photo-fission neutrons can play a large role in active NDA techniques [62], however in passive techniques such as DDSI it can largely be neglected. Photodisintegration can be an issue in certain light elements such as beryllium because it has a low photodisintegration threshold of $1.66 \mathrm{MeV}$. The DDSI instrument does not contain any significant amounts of beryllium, however, and thus it is not expected to be a significant source of neutrons due to photo-fission. 


\subsection{5 (n, xn) Neutrons}

Another potential multiplying source of neutrons are $(n, x n)$ reactions, where $\mathrm{x}$ is $\geq 2$. As with photofission, this reaction typically has a very high threshold $(\sim 10 \mathrm{MeV})$ and is rarely seen in spent fuel. Accounting for $(n, x n)$ neutrons could be important in situations where "neutrons are known to have high energy, when deuterium, beryllium, or tungsten are present, and when the coincidence count rates to be measured are very low" [1], however DDSI spent fuel measurement does not meet these requirements.

\subsection{Engineering and Physical Differences Between Fresh and Spent Fuel}

Because actual fresh fuel is used to experimentally test the DDSI analysis algorithms developed through this work, it is important to understand the differences between fresh and spent nuclear fuel. Differences exist both in the engineering aspects of the measurements as well as the physical processes at play in the assemblies. DDSI was designed for the engineering requirements of the spent nuclear fuel measurements; however it is also able to measure, to a certain degree, the simpler fresh fuel assemblies. Similarly, the physical aspects of spent fuel drove the development of analytical algorithms and the basic physical behavior of spent fuel can be recreated to a large extent in fresh fuel.

\subsubsection{Engineering Differences}

The primary difference between fresh and spent fuel assemblies from a detector system engineering standpoint is count rate. Typical spent fuel assemblies from PWRs passively emit on the order of $10^{8}$ neutrons and $10^{14}$ gamma rays per second. These particles are emitted along the entire length of the almost $4 \mathrm{~m}$ fuel rods, resulting in about $10^{7}$ neutrons and $10^{15}$ gamma rays available for detection at the $40 \mathrm{~cm}$ active length ${ }^{3} \mathrm{He}$ tubes. The resultant count rate for DDSI, given its neutron detection efficiency of approximately $12 \%$, is therefore on the order of $10^{6}$ neutrons per second. The gamma ray dose is also reduced significantly with a $5 \mathrm{~cm}$ lead shield surrounding the assembly and lead blocks within the stainless steel detectors pods. The gamma dose to the ${ }^{3} \mathrm{He}$ tubes is therefore expected to be on the order of $20 \mathrm{R} / \mathrm{hr}$. The instrument was designed with these expected detection and dope rates and utilizes multiple tactics to accommodate the high count rates. 
The large number of detectors, 56, helps to accommodate the high neutron count rate. The detector diameter of 1 " was selected to reduce the gamma pileup effects because of the faster pulse rise-time [63]. Each detector is paired with its own preamplifier to reduce deadtime. The cadmium lining around each detector pod also reduces the count rate along with its primary purpose of sensitivity to prompt fission neutrons rather than thermalized neutrons. The result of this is a reduced die-away time. Short time-constant preamplifiers are used to accommodate the high gamma count rate and avoid pileup issues, and ${ }^{3} \mathrm{He}$ detectors themselves have been shown to meet stringent gammarejection requirements [64]. A more detailed analysis of the preamplifiers' response to gamma radiation is given in Chapter 2 .

Storing the acquired data is a challenge when the count rate is so high. Pulses corresponding to detected neutrons may arrive too quickly to be converted and compressed and stored to RAM in real time. Therefore the TDMS file structure is used to store only a start time and a 0 or 1 depending on whether or not a pulse was detected in each sampling period. This is less computationally expensive than recording actual time after start on the fly. The TDMS files are compressed in post-processing, however, to eliminate the zeroes and recover the pulse arrival times.

\subsubsection{Physical Differences}

DDSI was designed for the characteristics and complexities of spent nuclear fuel assemblies, however much of what is observed with spent fuel can be recreated on a smaller scale in fresh fuel. The most important addition necessary for DDSI is a spontaneous fission source. Fresh fuel contains only low enriched uranium and therefore needs a neutron driver source in the assemblies. This can be solved by adding ${ }^{252} \mathrm{Cf}$ and/or ${ }^{244} \mathrm{Cm}$ sources to guide tubes to drive the assembly from within. The sources can be distributed axially and radially in the guide tubes to mimic the way the source is spread throughout the fuel pins in spent fuel.

Additionally, neutron absorbers such as gadolinium and cadmium play a large role in the fission chains by absorbing neutrons from the system and reducing the average neutron lifetime. This effect can be recreated in fresh fuel by adding LEU rods with gadolinium to introduce thermal neutron absorbers. The ${ }^{238} \mathrm{U}$ content is roughly the same between spent and fresh fuel, which leaves the final physical factor to consider: fissile material. 
The lack of plutonium also makes the fresh fuel considered in this work different than spent nuclear fuel, but ${ }^{239} \mathrm{Pu}$ and ${ }^{235} \mathrm{U}$ have very similar properties with the exception of the average number of neutrons released per fission. Therefore most of the properties of spent nuclear fuel relevant for assay by the DDSI instrument can be very closely mimicked with fresh fuel assemblies containing additional spontaneous fission sources and neutron absorber materials. 


\section{Chapter 4. Simulating THE DDSI INSTRUMENT RESPONSE}

The codes used to simulate RADs and the DDSI instrument are MCNP [58] and MCNPX-PoliMi [65]. MCNP was used for the majority of simulations. The code uses probability distributions and a wide variety of material cross-sections to predict the transport of various particles as they travel through matter. Monte-Carlo is a stochastic method and therefore requires a large number of particles to be simulated for the value of interest to converge with a sufficiently small variance. MCNP has been extensively tested and compared to various experiments to validate the capabilities of the tool for simulating detector systems such as DDSI [55].

\subsection{Simulation Methods}

RADs used in this work were simulated in two ways: 1) Construct a pulse-train from a PTRAC file created in an MCNP simulation (see section 4.1.2) and construct a RAD complete with accidentals, or 2) use a special capture tally (F8) in MCNP which records neutron captures in the detectors during a user defined time window and creates a reals-only RAD free of accidentals. The F8 capture tally method is preferable in many applications (such as this one) as it is far less computationally expensive because it can be run on large computer clusters to take advantage of parallel processing,

unlike PTRAC; however, it lacks the ability to implement dead-time, and thus may be limited in its usefulness for direct comparison to experimental measurements. It is shown in section 4.2.3 that the F8 method is a good simplification of the pulse-train method; the pulse train method is also more realistic due to the inclusion of accidental coincidences and deadtime.

\subsubsection{RADs from Simulated Pulse-Trains with MCNPX-PoliMi}

Pulse-trains can be simulated using the particle tracking, or PTRAC, feature of MCNP, or MCNPXPoliMi. It has also been done previously with MCNP-PTA [66]. PTRAC has two modes of 
importance to this problem: "general" and "capture". In the general mode, all features of individual neutron transport can be recorded, including collisions, fissions, surface crossings, and for each event, the time, location, direction of motion, and other relevant variables can also be listed and printed to an output PTRAC file. In the capture mode, only events in which captures take place in specified cells are recorded to the output file. Neutrons captured in the ${ }^{3} \mathrm{He}$ detection material are recorded along with their parent spontaneous fission (SF) isotope, the time of detection after the initial SF event, and the final isotope in the fission chain that led to the capture. The following chain of events would produce the corresponding output in the PTRAC capture mode:

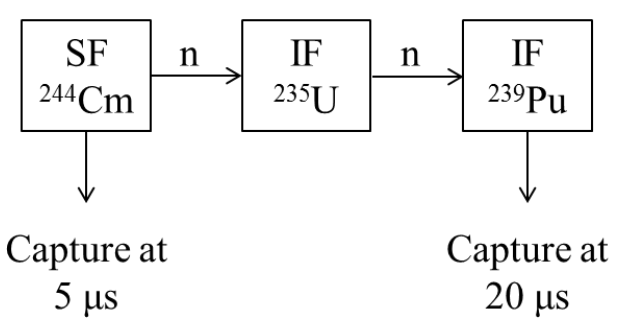

\begin{tabular}{llllll}
\hline Time [s] & SF Iso & 1st Fission & 2nd Fission & 3rd Fission & Last Fission \\
\hline $5 \cdot 10^{-6}$ & 96244 & 0 & 0 & 0 & 96244 \\
$2 \cdot 10^{-5}$ & 96244 & 92235 & 94239 & 0 & 94239 \\
\hline
\end{tabular}

In this case, a source $\mathrm{SF}$ event in ${ }^{244} \mathrm{Cm}$ initiated a short fission chain in which a neutron from the SF event went on to induce fission in ${ }^{235} \mathrm{U}$, then a neutron from that fission event went on to induce fission in ${ }^{239} \mathrm{Pu}$. Two neutrons from the fission chain were captured in a detector cell, one of which was from the SF event itself and was captured $5 \mu$ s after the release, and one of which was from induced fission in ${ }^{239} \mathrm{Pu}$ and was captured $20 \mu$ s after the spontaneous fission event. This is repeated for many histories until adequate statistics are obtained. Because all capture times are relative to the initial spontaneous fission event, the spontaneous fission event times must be distributed as if they were spread out over time as would be the case in a real sample. This is necessary to include the effect of accidental coincidences - capture events that come from other neutron creation histories that are not part of the original history.

The MCNPX-PoliMi code may be used to automatically time-distribute the SF events according to the source strength and record data for interactions within designated detector cells. Each capture 
interaction within the ${ }^{3} \mathrm{He}$ detectors is recorded and a data file is produced with detailed information on each event. For pulse-train development, the time of capture is the only necessary quantity to record. Because the source has been correctly distributed in time already, the capture times are spread appropriately and only need to be sorted to produce simulated list-mode data.

List mode data are converted into an RAD using the method described in Chapter 2. An example of an RAD produced from 600 seconds of simulated list-mode data from a ${ }^{252} \mathrm{Cf}$ source in the center of DDSI is shown in Figure 4-1 [8].

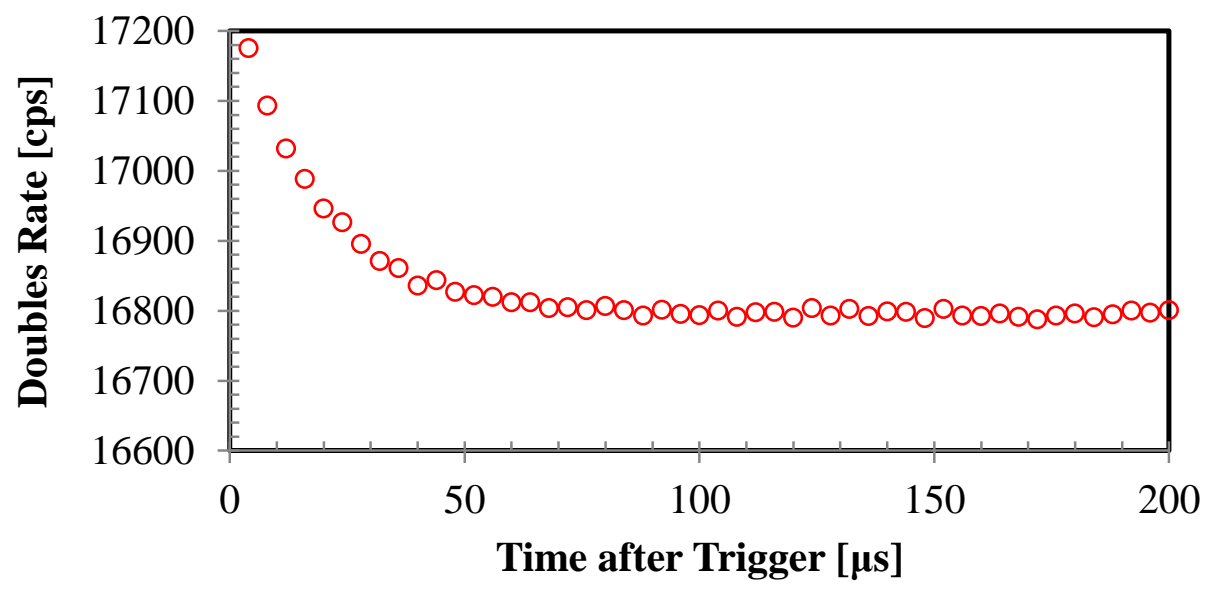

Figure 4-1. RAD produced with pulse-train data simulated with MCNPX-PoliMi.

The die-away behavior is clearly seen in this case. The curve is superimposed on a broad base of accidentals, which are defined as coincidences between uncorrelated neutrons. Accidentals should theoretically occur with the same frequency at all times after a trigger because, by definition, there is no true correlation between an accidental neutron arrival and the trigger neutron. Accidental detection rate can be determined in two ways: by counting neutrons arriving in a gate sufficiently long after the trigger that no true correlations should be present at that time, or from equation (1),

$$
A=S^{2} \times g
$$

where $A=$ accidentals rate, $S=$ detected singles count rate, and $g=$ gate width used to determine coincident neutrons [1]. The former method is considered preferable because it is obtained experimentally, and slight approximations are made in deriving the latter method, more details of which can be found in [1]. 
The pulse train method is beneficial for producing realistic RADs because they contain the accidentals component as would be observed in experiments, and because detector dead-time can be incorporated by setting a specified 'recovery' time-window after each trigger where no neutrons are counted in that detector. However, producing simulated pulse-trains is computationally expensive as simulations must be carried out in serial computations. For example, if the spent fuel assembly source rate is $1 \cdot 10^{8}$ spontaneous fission events per second (a common figure in spent nuclear fuel applications) then $6 \cdot 10^{9}$ event histories must be simulated to produce a pulse train equivalent to 1 minute of measurement. With a complex spent nuclear fuel source and system such as a DDS instrument, this simulation could take years of CPU time.

\subsubsection{Reals-Only RADs from F8 Capture Tallies in MCNP}

A much more effective method involves the use of sequential F8 capture tallies[8]. With the capture mode of an F8 tally, the user defines a pre-delay (PD) and gate width (GW). Once a neutron is captured, all subsequent neutrons arriving within the gate following the pre-delay are counted. By establishing uniform-width gates and sequential pre-delays, a RAD is produced. For example, a 200 $\mu$ s window RAD with $4 \mu$ s time bins is established by defining the following pre-delays and gates: $(\mathrm{PD}=0, \mathrm{GW}=4),(\mathrm{PD}=4, \mathrm{GW}=4),(\mathrm{PD}=8, \mathrm{GW}=4), \ldots,(\mathrm{PD}=196, \mathrm{GW}=4)$.

Each MCNP history begins with a single SF event; therefore all subsequent neutron production in that history must be correlated to that initial event. Because contributions to the tally are from a single history at a time, it is by definition free of accidentals. Without accidentals it is possible to obtain excellent statistics of real coincidences considerably faster than with the pulse-train method, however, the ability to add system dead-time and analyze real coincidences in the presence of accidentals is lost. An example of a reals-only RAD produced with the F8 capture tally method is shown in Figure 4-2. 


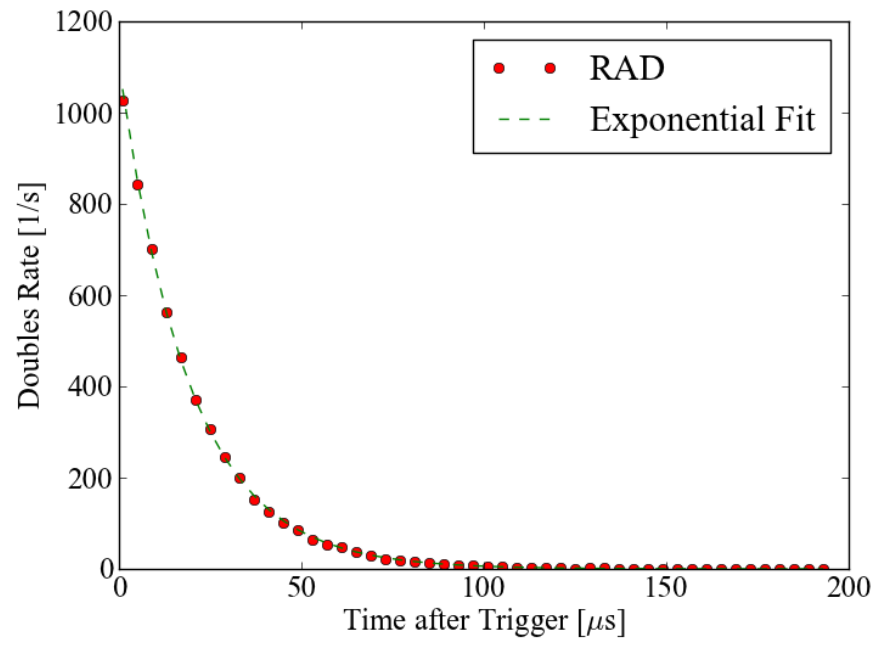

Figure 4-2. Reals-only RAD produced with F8 tally. The die-away time is $19.4 \mu \mathrm{s}$.

A rapid and accurate way to produce RADs is essential for the study of DDSI because the physics analysis and SFA characterization employed utilize RADs from tens to hundreds of different SFAs, each requiring a unique simulation.

\subsubsection{Comparison of Pulse Train and F8 Methods}

The PTRAC output was compared to an F8-produced reals-only RAD from the same simulation to confirm that the F8 approximation is accurate. The methods were compared by keeping only true coincidences (i.e. neutrons from the same MCNP history) from the pulse train and creating a histogram of capture times after trigger. The F8 RAD and pulse-train RAD from ${ }^{252} \mathrm{Cf}$ are plotted together in Figure 4-3. 


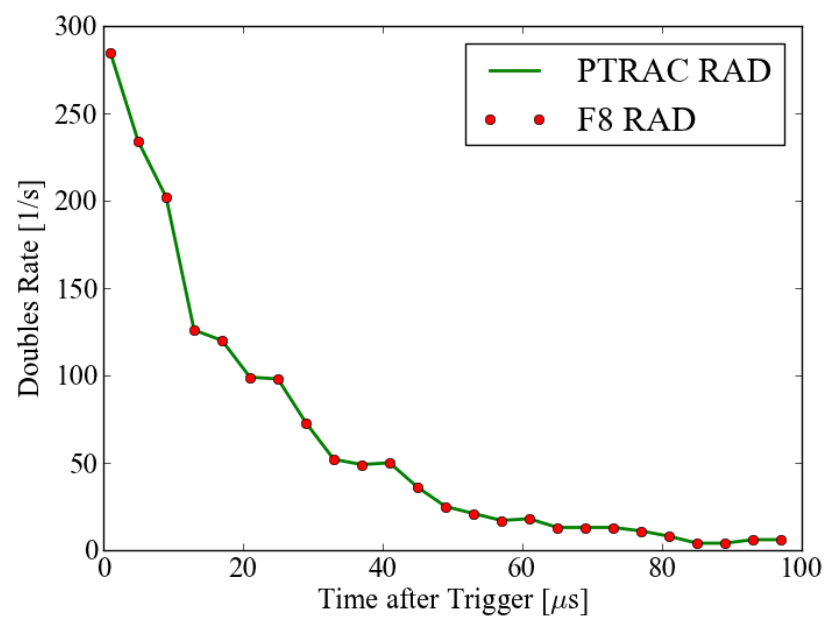

Figure 4-3. Reals-only RAD produced with PTRAC output compared to RAD from F8 tally. The agreement is exact indicating that the F8 RAD is identical to the PTRAC output when accidentals are omitted.

The identical agreement indicates that the assumptions made regarding the operation of the F8 RAD production are correct, and the F8 capture tally method is an accurate representation of the pulse train with no accidentals. Thus moving forward, the F8 capture tally method was used to efficiently simulate spent and fresh fuel assembly reals-only RADs.

\subsection{Incorporating Fuel Models into DDSI}

As discussed in the previous chapter, spent fuel libraries (SFLs) provide material descriptions for a variety of assemblies with variations in BU, IE, and CT, among others. The assemblies from SFLs as well as the fresh assemblies considered are defined on a pin-by-pin basis. The DDSI instrument is simulated in great detail (see Appendix C) in a pool of water and the assemblies are simulated in the center of the instrument in all dimensions. It is not necessary to model source emissions from the entire length of the assemblies because the pins are almost $4 \mathrm{~m}$ long while the detectors have only a $40 \mathrm{~cm}$ active length. Therefore the contributions from the pins reduced significantly beyond approximately $45 \mathrm{~cm}$ in either direction, as shown in Figure 4-4. 


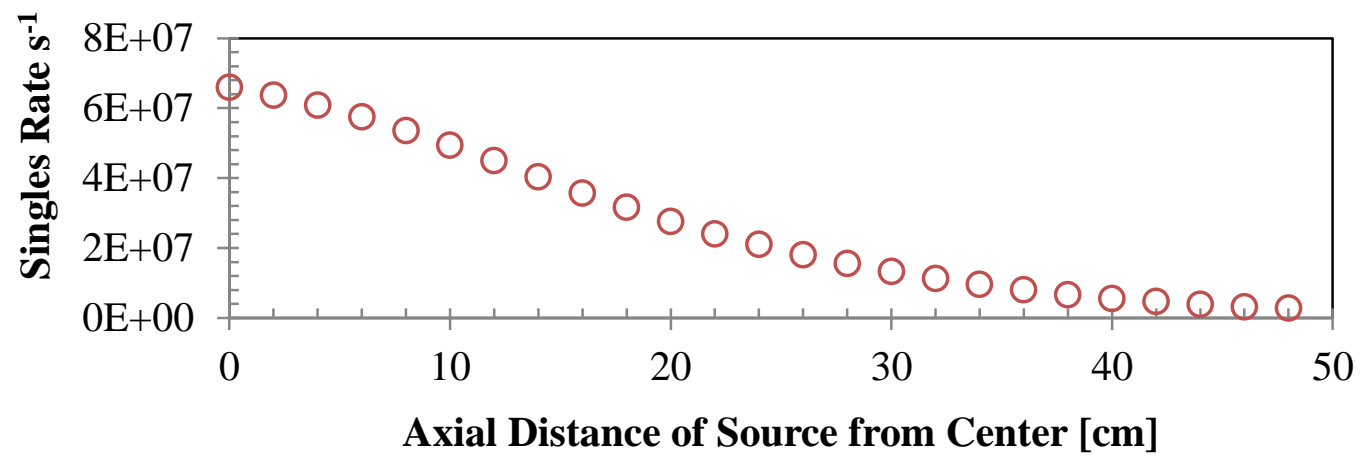

\section{Figure 4-4. Singles rate in detectors as a function of axial distance of source emissions from center of the $45 \mathrm{GWd} / \mathrm{tU}$ BU, $4 \% \mathrm{IE}$, and 5 y CT assembly.}

The signal from $44-50 \mathrm{~cm}$ constituted $1.4 \%$ of the signal over the entire $50 \mathrm{~cm}$, indicating that $44 \mathrm{~cm}$ in each direction was a reasonable extent of the assembly to use for the simulations. This is approximately $1 / 4$ of the entire length of the fuel rods.

\subsection{Statistics and Uncertainty}

One of the barriers to any coincidence or multiplicity-based technique for assaying spent and fresh fuel is the large impact of accidental coincidences. Accidentals are defined as any detection event measured in a coincidence window that is uncorrelated to the trigger event. This includes neutrons from other fission events happening in the fuel simultaneously, as well as neutrons from $(\alpha, n),(n$, $2 n$ ), and any other independent reactions. In some cases with high count rates, the accidental coincidences may be as much as 4000 times more prominent than true coincidences in the time region of interest in the RAD. This therefore requires sufficiently high statistics to obtain a usable signal to background ratio.

The signal can be defined in several ways with the DDSI instrument. The definition is given in equation (4)

$$
\text { Signal }=\int_{5}^{\tau} D d t
$$

where $D=$ doubles and $\tau=$ die away time. The starting time of $5 \mu$ s is used to allow time for a predelay to reduce deadtime effects. This formula can be applied to the fast or slow components or the 
early die-away region of the RAD. The Signal to background ratio ranges over SFL2a for these three components are $0.003-0.011,0.003-0.010$, and $0.006-0.017$, respectively. The range of ratios is shown in Figure 4-5. The best ratios are consistently found with the early die-away time which makes sense because it is the highest magnitude signal. The signal/background ratio gets worse as a function of accidentals count rate which is also to be expected.

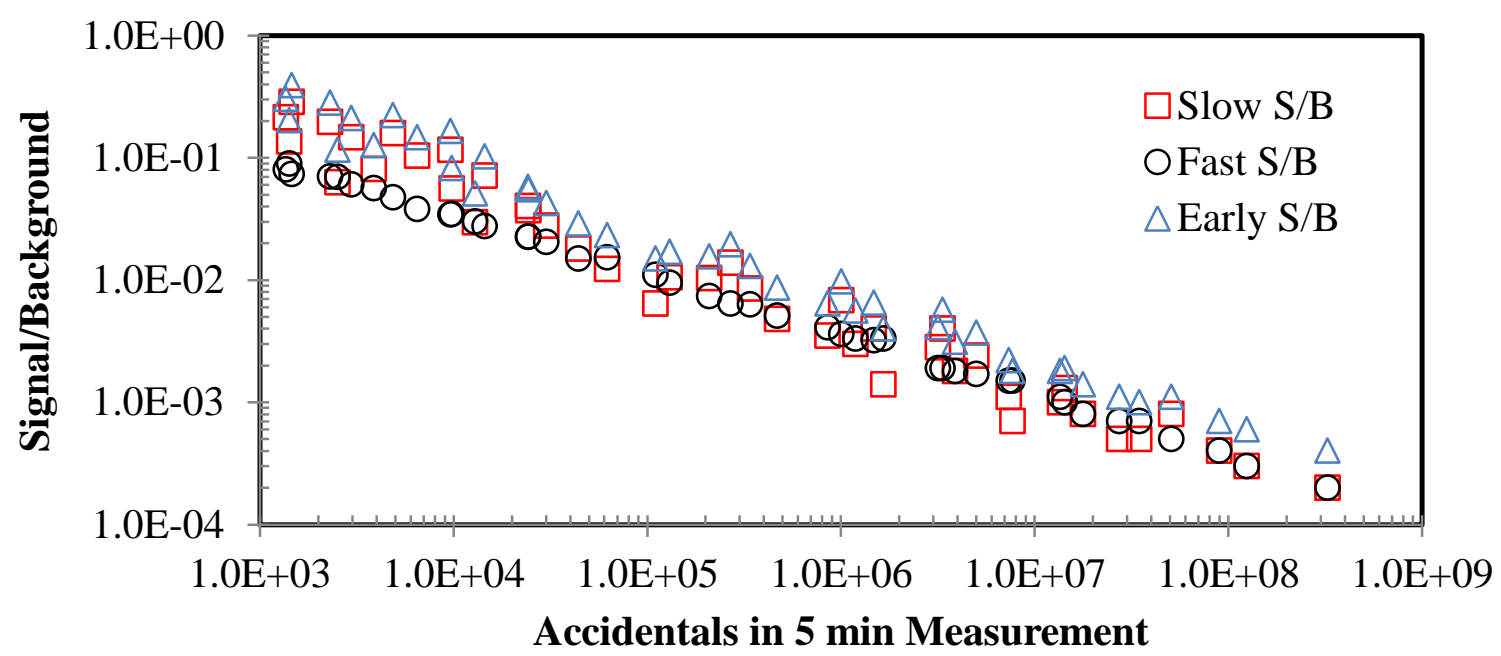

Figure 4-5. Signal/Background (S/B) ratio for slow and fast components and early die-away region. The $\mathrm{S} / \mathrm{B}$ ratio is consistently favorable for the early die-away region because of the higher magnitude of the signal.

In experiments, accidentals will be obtained from shift register analysis because finding accidentals from the RAD is far more computationally expensive. The accidentals are needed in order to accurately determine the uncertainty in the die-away times calculated from fits to the RAD. Not accounting for additional uncertainty that would arise in experiments, if the accidentals gate began after the reals dropped to less than $1 \sigma$ above accidentals, that would require the RAD to extend out to as long as $800 \mu$ s according to simulations, as shown in Figure 4-6. In order to avoid extending the RAD out to $800 \mu$ s in order to determine the accidentals, the familiar $A=T^{2} \cdot G$ will be used. Thus, it is sufficient to compute RADs out to 200-300 $\mu$ s in order to determine the early die-away time which will be considerably faster to implement in the RAD production algorithm. 


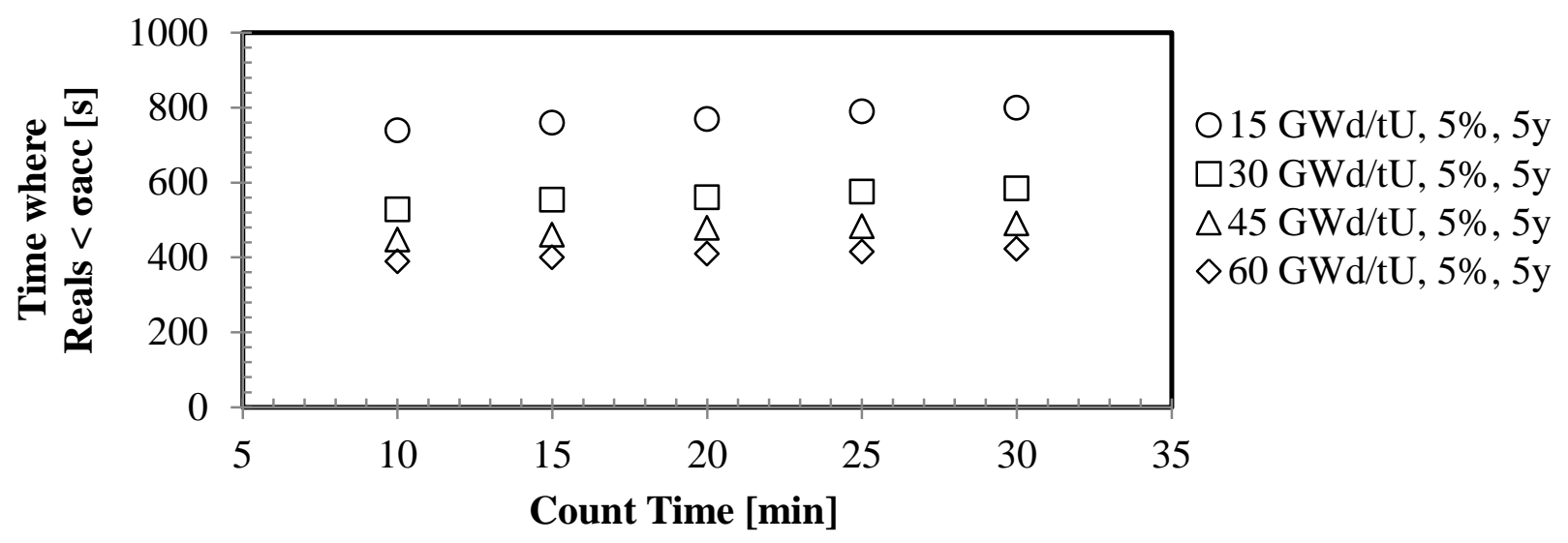

Figure 4-6. Time where reals fall lower than 16Accidentals for four assemblies of interest. These represent the limits of where the accidentals gate can be measured. To accommodate the low count rate 5/15/5 case, at least $800 \mu$ s would need to precede the accidentals gate.

Die-away times are quoted throughout this work and determining the uncertainty on a die-away time is useful for both simulated and experimental RADs. The uncertainty in the die-away time is determined by propagating the uncertainty from each doubles rate data point that makes up the RAD into the non-linear least squares fitting algorithm, and the final uncertainty is determined from the covariance matrix of the fit. Uncertainty on each point on the RAD is determined using 10 minute measurement times to represent an upper end of statistical uncertainty. Doubles rate uncertainty is given in equation (5)

$$
\sigma_{\text {Doubles }}=\frac{\sqrt{(R+A)+A}}{t}
$$

where $\sigma_{\text {Doubles }}=$ uncertainty in doubles rate $R+A=$ counts in a RAD bin, $A=$ accidentals, and $t=$ count time.

\subsection{Benchmark of the Detector Pod Model}

Before the DDSI instrument was assembled in its entirety, a simple experiment was conducted to compare with simulations of one detector pod to ensure that the simulated geometry is accurate. A $0.489 \mu \mathrm{Ci}{ }^{252} \mathrm{Cf}$ source was affixed to the center of the detector pod face. Ten detectors (shown in red in Figure 4-7) were used for data collection in fast mode, and data were collected for 10 minutes. Data were then converted to a Rossi-alpha distribution via FastTapX and the RAD was analyzed for die-away time and singles rate with Python scripts. The simulation was made to match the experimental setup as closely as possible. Very slight changes were made to the current DDSI 
detector pod simulation and these changes were carried over to the full instrument simulation to improve its accuracy.

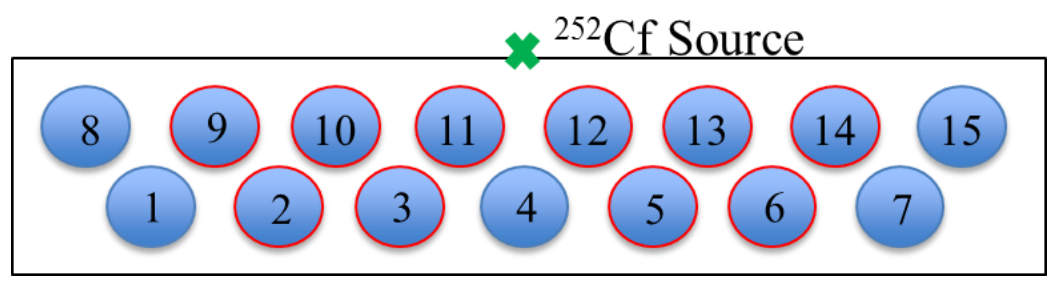

Figure 4-7. Detector configuration for benchmarking measurements. Detectors outlined in red were used in the experiment.

The RAD with accidentals subtracted from the experiment is on the top left of Figure 4-8, and the reals-only RAD from simulation is on the right. The overlain comparison is on the bottom.

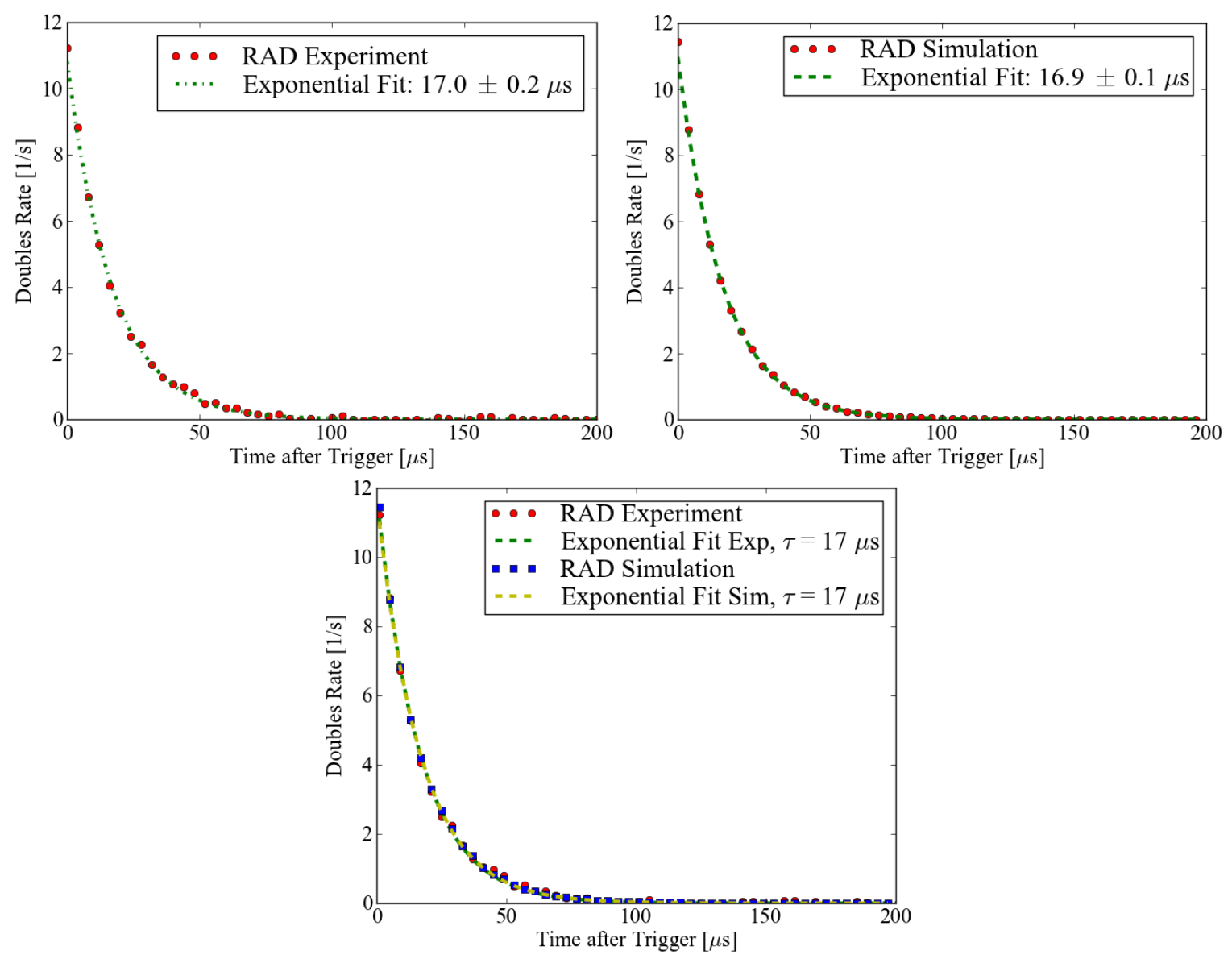

Figure 4-8. Experimental RAD with accidentals subtracted (top left), simulated reals-only RAD (top right), and comparison (bottom). Agreement is very good both in magnitude and doubles rate. 
Both the die-away times and count rates agree very well, as shown in the summary Table 4-I. Agreement in the die-away times and singles rates were both within 1\%. Experimental results are deadtime corrected.

Table 4-I. Summary of experiment and simulation RAD analysis results.

\begin{tabular}{cccc}
\hline & Die-Away Time [ $\boldsymbol{\mu s}]$ & Singles Rate [cps] & Doubles Rate [cps] \\
\hline Experiment & $17.0 \pm 0.2$ & $775.231 \pm 0.001$ & $40.33 \pm 0.01$ \\
Simulation & $16.9 \pm 0.1$ & $768.046 \pm 0.002$ & $40.31 \pm 0.02$ \\
Percent Difference & $0.60 \%$ & $-0.90 \%$ & $-0.05 \%$ \\
\hline
\end{tabular}

There are several other factors in addition to simple counting statistics that will add uncertainty to the experimentally obtained RAD. Deadtime losses, temperature and high voltage drifts, and changes in the experimental setup such as shifted assemblies could all contribute to the uncertainty in the signal and accidentals. 


\section{Chapter 5. Analysis of Simulated Spent Fuel Assembly Assay}

Safeguarding spent nuclear fuel through nondestructive assay is particularly challenging because spent fuel is one of the most complex special nuclear materials in existence. The fuel contains several hundred different isotopes that may interact with neutrons in different ways. Fissile isotopes add to the neutron population through induced fissions while neutron absorbers remove from the neutron population through captures. Because these effects cannot be observed individually in fuel measurements, simulations are a useful tool with which to improve our understanding of the influence of individual spent fuel isotopes and interactions as well as to develop analysis algorithms that can later be tested in experiments. Hundreds of assays of simulated spent nuclear fuel assemblies were used to understand the behavior of the DDSI instrument and its ability to characterize spent nuclear fuel.

\subsection{Leakage Multiplication Determination}

Neutron interactions in a material that produce additional neutrons are considered multiplying events. Quantifying the amount of multiplication in a system is essential for certain methods to determine effective fissile mass, neutron absorber effects, and elemental Pu mass, among other quantities. In DDSI, the measured signal is the result of the source neutrons multiplied by the system's net multiplication, and thus multiplication must be understood in order to draw quantitative connections between the source and the measured signal.

\subsubsection{Definitions of Multiplication}

Leakage multiplication may be considered approximately equal to net multiplication under certain conditions, however it is not true universally and it is important to consider the implicit assumptions. 
To begin, various forms of multiplication are derived and defined including total multiplication, leakage multiplication, and net multiplication.

The most fundamental definition of multiplication is simply the average number of neutrons produced from a single starting neutron. This is derived by considering first that single neutron is created in generation 0 . In generation 1 , that neutron caused $p$ fissions where $p$ is the probability of fission. Each fission produced $v$ neutrons, resulting in the total production of $p v$ neutrons. However the first neutron was absorbed in order to create fission, so the net gain in generation 1 is $\mathrm{p}(v-1)$. Each of the $p v$ neutrons produced goes on to cause $p$ fissions, each producing $v$ neutrons, resulting in the total production of $\mathrm{p} v(\mathrm{pv})=(\mathrm{p} v)^{2}$ neutrons. However, again, loss of neutrons to fission is accounted for resulting in a net gain in generation 2 of $p v(v-1)$ neutrons. The neutrons produced and net gain for the first several generations are listed in Table 5-I.

Table 5-I. Neutrons produced and net gain in neutrons for first several generations of a fission chain.

\begin{tabular}{lll}
\hline Generation & Neutrons Produced & Net Gain \\
\hline $\mathbf{0}$ & 1 & 1 \\
$\mathbf{1}$ & $\mathrm{p} v$ & $\mathrm{p}(v-1)$ \\
$\mathbf{2}$ & $\mathrm{p}(\mathrm{p} v) v=(\mathrm{p} v)^{2}$ & $\mathrm{p}(\mathrm{p} v)(v-1)$ \\
$\mathbf{3}$ & $\mathrm{p}(\mathrm{p} v)^{2} v=(\mathrm{p} v)^{3}$ & $\mathrm{p}(\mathrm{p} v)^{2}(v-1)$ \\
$\mathbf{4}$ & $\mathrm{p}(\mathrm{p} v)^{3} v=(\mathrm{p} v)^{4}$ & $\mathrm{p}(\mathrm{p} v)^{3}(v-1)$
\end{tabular}

To derive total multiplication, the total number of neutrons produced in all generations is added which is a simple geometric summation:

$$
M_{\text {total }}=1+p v+(p v)^{2}+(p v)^{3}+(p v)^{4}+\cdots=\sum_{n=0}^{\infty}(p v)^{n}=\frac{1}{1-p v}
$$

If instead the net production of neutrons, i.e., neutron profit per source neutron, is of interest, the third column of net gain in Table 5-I is summed instead: 


$$
M=1+\sum_{g=0}^{\infty} p(v-1)(p v)^{g}=1+p(v-1) \sum_{g=0}^{\infty}(p v)^{g}=1+\frac{p(v-1)}{1-p v}=\frac{1-p}{1-p v}
$$

MCNP defines net multiplication as unity plus the net gain in neutrons from multiplying reactions including fission, $(\mathrm{n}, 2 \mathrm{n})$, and all other multiplicative reactions in the system. This is the equivalent of the net production of neutrons above and therefore the same final expression as in equation (7) can be used to define net multiplication in MCNP. However, one may observe that the net neutrons produced from fission is not the same as the net neutrons available for counting; neutrons produced from fission may either leave the source region and have the possibility of being counted, or they may be absorbed in the source region and thus not be available for counting. Therefore if the multiplication as it applies to neutron counting is of interest, i.e., net neutrons produced per source neutron and available for counting, the net multiplication summation must be adjusted as follows to give the leakage multiplication factor:

$$
M_{L}=\frac{1-p}{1-p v} \times \frac{p_{L}}{p_{L}+p_{c}}
$$

where $p_{L}=$ probability of leakage and $p_{c}=$ probability of capture in source region. If the previous assumption that the only possible courses of action for a neutron are fission, leakage, or absorption is utilized, then $1-p=p_{L}+p_{c}$. This yields the familiar form of leakage multiplication:

$$
M_{L}=\frac{p_{L}}{1-p v}
$$

To summarize the definitions:

Total Multiplication $=\frac{1}{1-p v} \quad$ Leakage Multiplication $=\frac{p_{L}}{1-p v} \quad$ Net Multiplication $=\frac{1-p}{1-p v}$

The most critical result of these derivations is that the leakage multiplication can only be considered approximately equal to the net multiplication as defined by MCNP if the probability of capture is negligible compared to the probability of leakage. In the case of typical neutron counters this is not an issue because the detectors surround the assayed item resulting in a very high probability of leakage into the detector region. Therefore leakage and net multiplication are often used interchangeably in safeguards applications. 


\subsubsection{Traditional Leakage Multiplication Determination Methods}

(i) Doubles/singles ratio

There is no independent method by which multiplication can be determined experimentally. It can be computed with Monte Carlo codes as is done for spent fuel assemblies, calculated with the point model equations and triples and doubles rates, or the most common method is estimation using the observed doubles/singles ratio [67, 68]. The complete derivation is given in the Ensslin paper [67]. The method utilizes the fact that sample self-multiplication affects neutron coincidence counting more than it does total neutron counting, so the ratio of doubles/singles increases with multiplication. An expression for multiplication is derived using the fission moments derived by Boehnel [69], and can then be expressed as a function of the spontaneous and induced fission multiplicities and the $\alpha$ ratio defined as the number of $(\alpha, n)$ neutrons over the number of spontaneous fission neutrons. This method has been used for several decades and provides reliable estimates of multiplication, however in a source with a non-negligible $(\alpha, n)$ component, the $\alpha$ ratio must either be known or assumed calculable. The method also assumes the "superfission concept", i.e. that all spontaneous and induced fission chains are simultaneous within the resolving time of the coincidence counter [70]. This concept is not valid for the DDSI instrument in which measured fissioning sources typically have longer die-away times than the detector itself, resulting in fission events that cannot be assumed to be simultaneous.

(ii) Late gate / early gate ratio

Previous studies of the DDSI instrument circa 2010 theorized, based on simulations, a relationship between the ratio of the doubles in a late gate to doubles in an early gate $(\mathrm{L} / \mathrm{E})_{\mathrm{D}}$ and fissile content [10]. The smooth trends of the $(\mathrm{L} / \mathrm{E})_{\mathrm{D}}$ ratio and burnup and initial enrichment were put forth as evidence of this correlation. It was proposed that the ratio be used along with D/S to predict assembly multiplication. This concept was adopted in 2015 by the Japan Atomic Energy Agency for use with a DDSI-based instrument to measure fuel debris from the Fukushima Daiichi nuclear power plants [71]. Nagatani et al. demonstrate a relationship in simulation space between the $(\mathrm{L} / \mathrm{E})_{\mathrm{D}}$ ratio and multiplication of measured debris, as shown in Figure 5-1. 


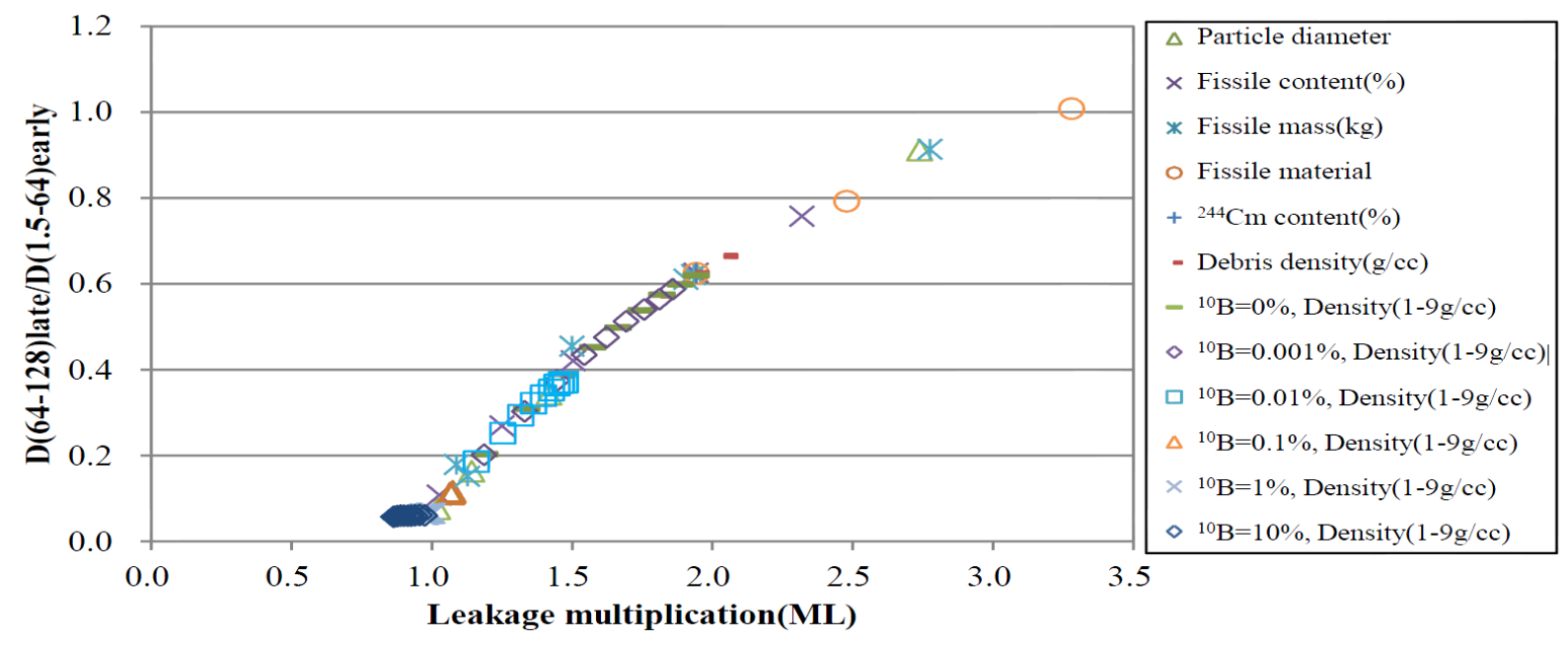

Figure 5-1. Application of $(\mathrm{L} / \mathrm{E})_{D}$ measurement to Fukushima fuel debris, from [71]

As is discussed later in the same paper, the primary drawback of this method is that the different gates have varying sensitivity to absorption and multiplication. This results in a trend that is no longer smooth when neutron absorbers and fissile content are not present in the same effective ratio. Measured data presented in the same paper documented this behavior. By utilizing the die-away time rather than gates, it is theorized that this effect could be avoided because the die-away time encapsulates the change in the signal both in the early and late time domains. The $(\mathrm{L} / \mathrm{E})_{\mathrm{D}}$ ratio, however, is affected by the timescale of the different processes, being absorption and multiplication. Therefore a new method described in the next section is proposed that improves upon this $(\mathrm{L} / \mathrm{E})_{\mathrm{D}}$ ratio approach that allows determination of multiplication regardless of the ratio of absorbing/fissile isotopes in the fuel assembly.

\subsubsection{Utilization of the RAD and Early Die-Away Time Concept}

Because of the rapid die-away of the fast component, the RAD in later time domains (>100 $\mu \mathrm{s})$ is shaped almost entirely by its slow component. RADs in the early time domains, however, have a dieaway time that is given by the sum of its fast and slow components at those times. The shape of RADs in the early time domain simulated for four different SFAs from SFL2a is shown in Figure 5-2 to demonstrate the effect of the changing fast/slow ratio on the RAD as a whole. The RADs in Figure 5-2 have $4 \mu$ s time bins and a non-linear least squares fitting algorithm was used to determine the parameters of the exponential functions representing both of their components. The RAD time window simulated is $200 \mu \mathrm{s}$. 

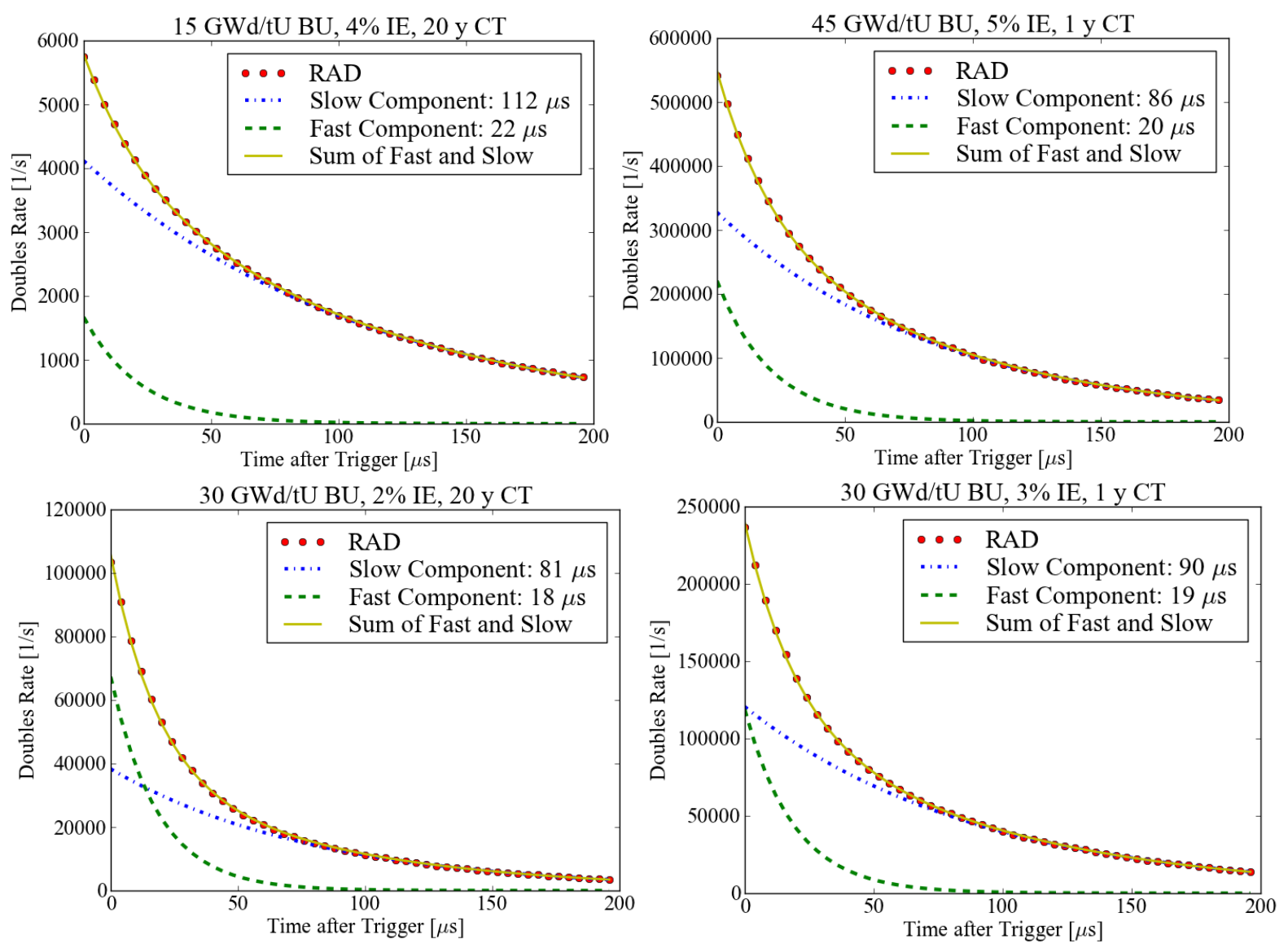

Figure 5-2. Comparison of four SFAs with various values of IE, BU, and CT. The relative magnitudes of the fast and slow components shape the die-away time of the total RAD in the early time domain. The statistical uncertainties on the RAD values are smaller than the markers used.

The relative magnitudes of the two components contain information about the fissile content of the assembly. Low initial enrichment SFAs have generally less fissile mass, and therefore they have a weaker slow component because fission chains do not occur or propagate easily. This also results in a lower net multiplication. The relationship between these system parameters allows us to determine multiplication from the RAD directly. Assemblies with relatively more neutron absorbers also generally have a weaker slow component because of the ability of the absorbers to suppress the evolution of induced fission chains; however, these assemblies also have high fissile content resulting in a larger magnitude fast component. These effects shape the die-away of the early time domain differently than low fissile mass assemblies so that they may be distinguished in analysis of the RAD. 
An exponential function can be fit to the early time domain of a simulated RAD as shown in Figure 5-3. The early die-away time is the decay constant of this function.

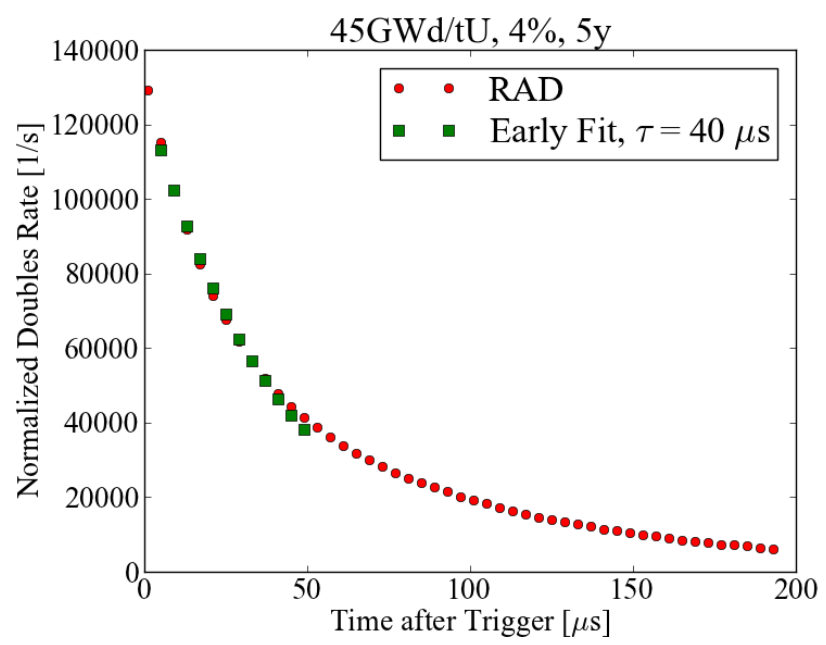

Figure 5-3. Exponential fit of the early time domain of an RAD shown with squares. The earlydie away time is determined from this function. In this example, it is $40 \mu s$. The uncertainties on the RAD values are smaller than the markers used.

The die-away time of the RAD in the early time domain as well as that of its slow component vary widely over the 44 assemblies in SFL2a, though the die-away time of the fast component remains statistically constant around $19 \pm 1 \mu$ s, as expected.

The multiplication of the entire setup with each individual assembly is also determined by MCNP. The code considers the entire system for determination of multiplication rather than just the fuel, but considering the minor contributions of multiplying reactions outside the fuel, and the similarity of these contributions across the 44 assemblies, it is assumed that the MCNP computed multiplication is an accurate approximation of the true leakage multiplication of the assayed SFA (as discussed in Section 5.1.1), and an accurate representation of the relative change in multiplication between SFAs.

\subsubsection{Role of the Alpha Ratio}

The original development and analysis of DDSI instrument performance determined multiplication through utilization of the doubles/singles ratio [72]. This is a commonly utilized method in passive neutron coincidence-based measurements of plutonium [1], that relies on the ability to correct for the effect of $(\alpha, n)$ neutrons. The method fits a second order polynomial to the ratio of the total coincident counts in a pre-defined doubles gate, typically between $\sim 24-64 \mu$ s, to the total observed 
counts (singles). This method assumes that the alpha ratio can be calculated, which is rarely if ever true for spent fuel.

In spent fuel, SF and $(\alpha, n)$ events develop fission chains in the same way (with the $(\alpha, n)$ neutronstarted chain being effectively just one fission generation shorter). As a consequence, unlike the D/S ratio, the die-away time of the RAD in the early time domain remains unchanged even if $(\alpha, n)$ neutrons are included in the simulation as documented in Table 5-II for the case of a SFA with the highest $\alpha$-coefficient among those in SFL2a (e.g. SFA with 5\% IE, $15 \mathrm{GWd} / \mathrm{tU}$ and 80 y of CT).

Table 5-II. Change in $\tau_{\text {early }}$ and D/S factors with different neutron sources.

\begin{tabular}{cccc}
\hline & SF Source Only & $(\boldsymbol{\alpha}, \mathbf{n})$ Source Only & Combined Sources \\
\hline $\boldsymbol{\tau}_{\text {early }}[\boldsymbol{\mu} \mathbf{s}]$ & 86 & 86 & 86 \\
$\mathbf{D} / \mathbf{S}$ & 0.28 & 0.18 & 0.21 \\
\hline
\end{tabular}

The early die-away time does not change within statistical and fitting uncertainty with the choice of either SF or $(\alpha, n)$ as the driving interrogation source. The D/S, however, changes significantly. Thus it is not surprising that the $\mathrm{D} / \mathrm{S}$ ratio does not produce a smooth second order polynomial without the knowledge of an additional parameter to accurately predict multiplication. The insensitivity of the early die-away time to $(\alpha, n)$ contributions makes it advantageous for ease of calibration and to reduce the necessary prior knowledge.

\subsubsection{Results for Different Spent Fuel Libraries}

(i) SFL2a

The multiplication of the SFAs in the 55 assemblies of SFL2a as a function of early die-away time is shown in Figure 5-4. $1 \sigma$ uncertainties are shown for the early die-away time values. Uncertainties are on the order of $1 \mu \mathrm{s}$. 


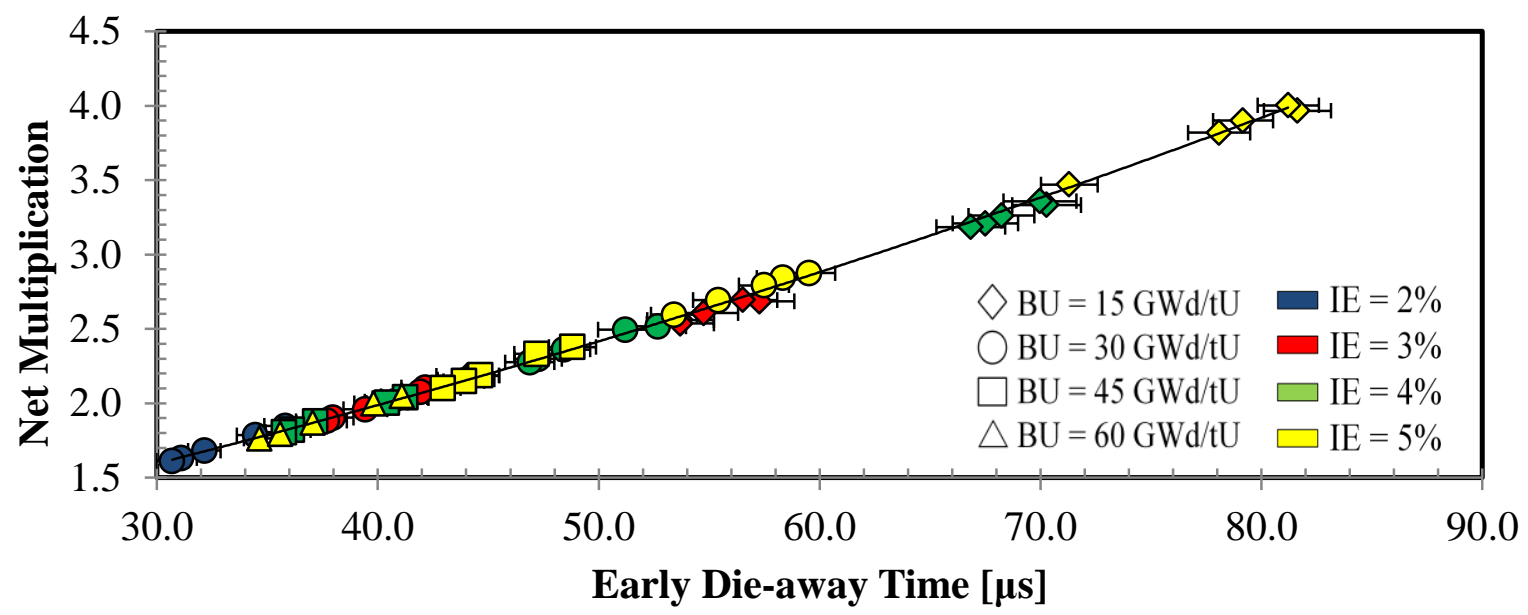

Figure 5-4. Net multiplication as a function of early die-away time calculated by fitting an exponential to the early time domain of a simulated RAD. A linear fit is applied with a slope of $0.05 \mu$ s. The $1 \sigma$ uncertainties on the early die-away time are given. Uncertainties on the net multiplication values are smaller than the markers used.

Figure 5-4 indicates a correlation between the multiplication of an assembly and the early die-away time, regardless of the combination of IE, BU, and CT. Both a linear and a $2^{\text {nd }}$ order polynomial fit can be applied to the data, though theoretically a $2^{\text {nd }}$ order polynomial should be a better fit of the data because of the relationship between multiplication and the doubles rate. The differences in prediction of net multiplication and calculated net multiplication for each assembly are shown in Figure 5-5.
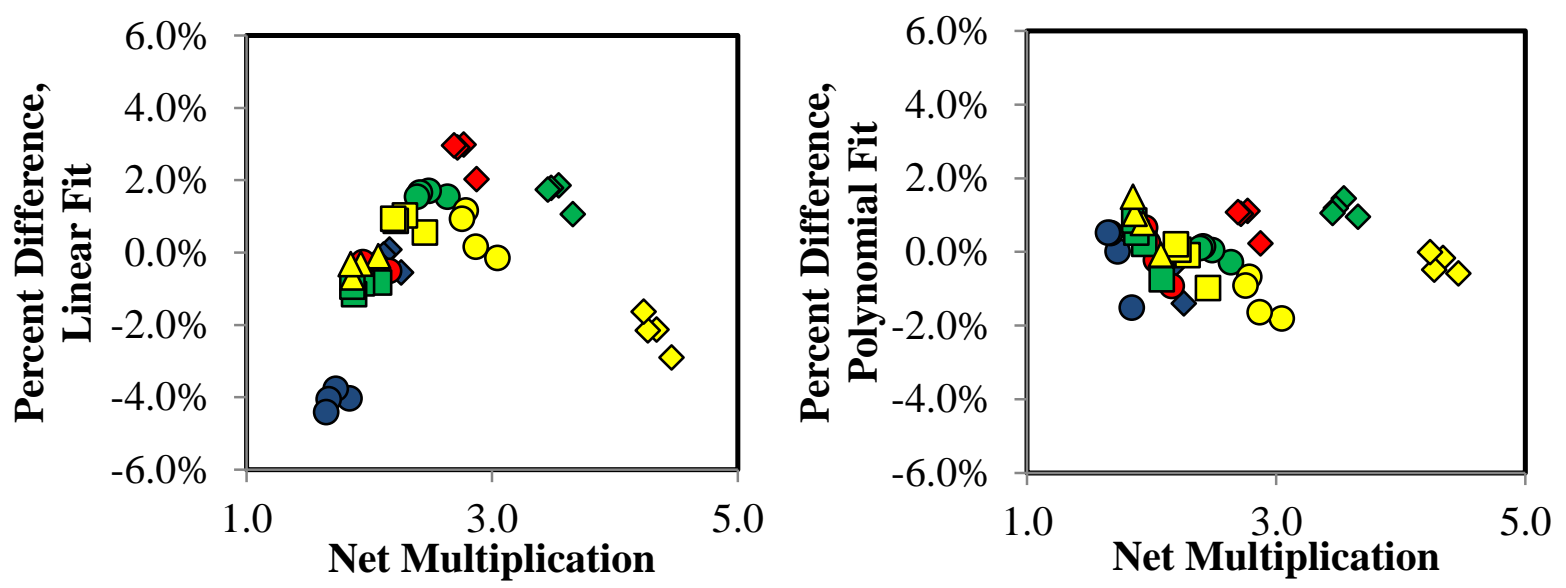

Figure 5-5. Relative difference in predicted and computed net multiplication for 44 SFAs from SFL2a. Linear fit (top) and 2nd order polynomial fit (bottom). Mean variation is 1.4\% for the linear fit and $0.7 \%$ for the $2^{\text {nd }}$ order polynomial. The legend is the same that was used for Figure 5-4. From the published work [9]. 
The distribution of the relative differences in Figure 5-5 indicates that a polynomial fit rather than linear will provide better results. The second order polynomial produced an average variance of $0.7 \%$, which is better than the linear fit. The average difference of $0.7 \%$ represents a limit of the accuracy of the method from its physics principle. The calibration curve determined with SFL2a is henceforth used to determine multiplication in assemblies that are considered "unknown" such as missing pin scenarios (discussed in section 5.3) and SFL4 mystery assemblies.

(ii) SFL3

As discussed in section 3.2, SFL3 was created at Oak Ridge National Laboratory and was a sensitivity study on operating parameters. Table 5-III describes the different parameters varied in SFL3.

Table 5-III. Description of operating parameters varied in SFL3 sensitivity study.

BAA Burnable absorber rods inserted into 24 guide tube locations

Base Base case: infinitely reflected 17x17 PWR assembly with 4 radial fuel regions, 4\% IE

Mod62 Moderator density of $0.62 \mathrm{~g} / \mathrm{cc}$

Mod 67 Moderator density of $0.67 \mathrm{~g} / \mathrm{cc}$

Mod 77 Moderator density of $0.77 \mathrm{~g} / \mathrm{cc}$

Mod 82 Moderator density of $0.82 \mathrm{~g} / \mathrm{cc}$

For each variation in operating parameter, burnup values of 30 or $45 \mathrm{GWd} / \mathrm{tU}$ were simulated as well as cooling times of 1, 5, 20, and 80 years. In analyzing this library of assemblies in DDSI, the goal was to determine whether altering these operating parameters changed the measured DDSI signal in such a way that the analysis algorithms developed no longer accurately characterized the assemblies. In the case of early die-away time to determine multiplication, it was observed that multiplication was accurately determined using the calibration curve developed on SFL2a assemblies with a standard deviation of 1.1\%. Results are shown in Figure 5-6 and Figure 5-7. MCNP reported Net Multiplication vs. early die-away time is shown first as a function of operating parameter, then as a function of burnup. $1 \sigma$ uncertainties are shown assuming 10 minute measurements. Uncertainties are on the order of $1 \mu \mathrm{s}$. 


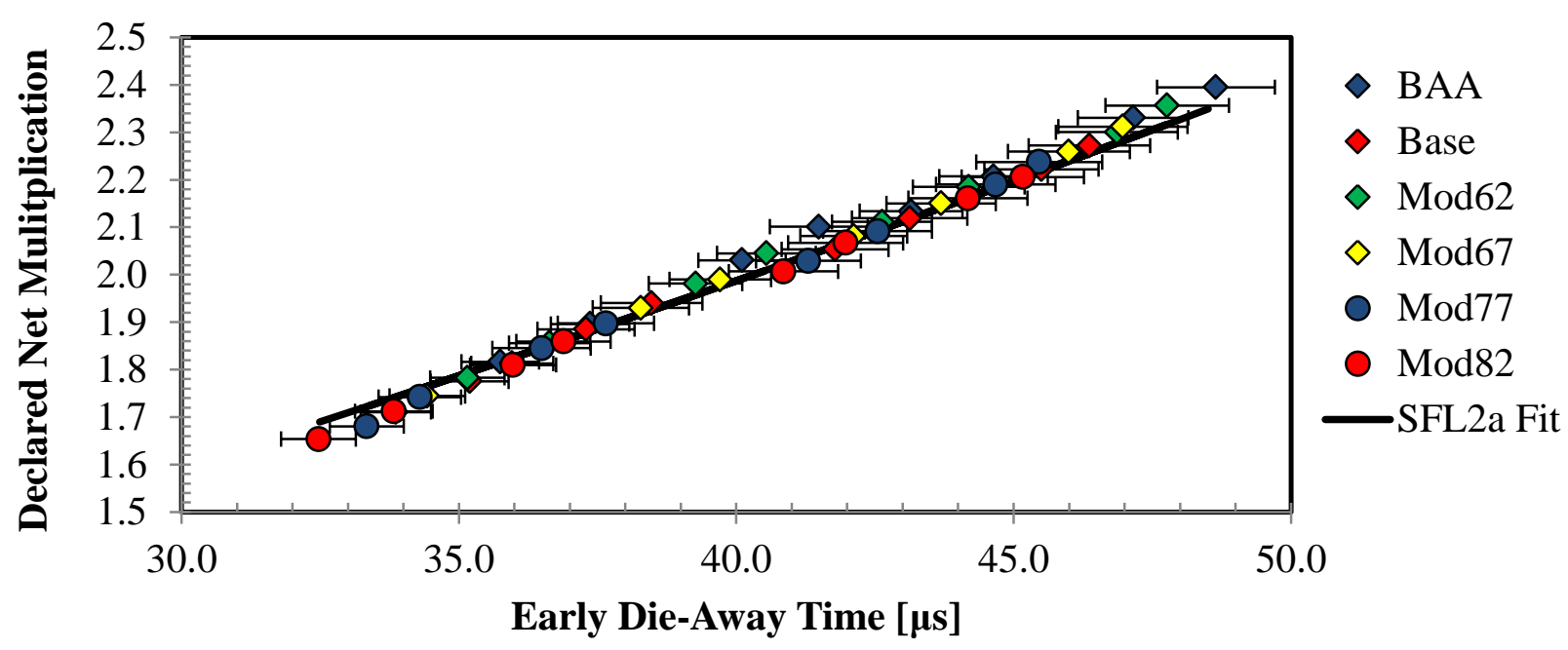

Figure 5-6. Multiplication vs. early die-away time, as a function of operating parameter. Fit from SFL2a is shown with a solid line. The $1 \sigma$ uncertainties on the early die-away time are given. Uncertainties on the net multiplication values are smaller than the markers used.

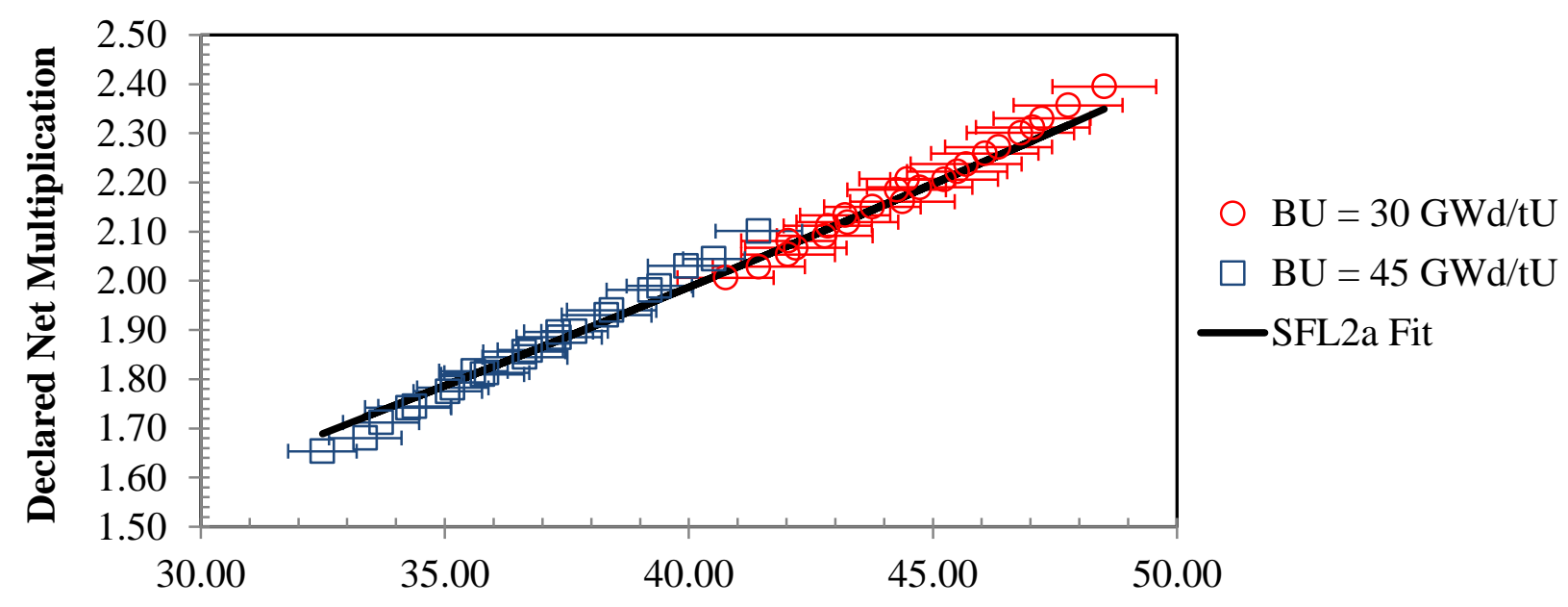

Early Die-Away Time $[\mu s]$

Figure 5-7. Multiplication vs. early die-away time as a function of burnup. Fit from SFL2a is shown with a solid line. The $1 \sigma$ uncertainties on the early die-away time are given. Uncertainties on the net multiplication values are smaller than the markers used.

Figure 5-6 demonstrates that varying operating parameters does not skew the multiplication vs. early die-away time relationship. The near-linear trend is still observed, and multiplication is determined very accurately using the SFL2a fit. There is a break in the data, however, that appears to form two separate lines or curves. Figure 5-7 clearly shows that this break is due to burnup, not operating parameters. This could be the result of a few different issues that are outside the scope of this work. 
(iii) SFL4

SFL4 contains the "mystery" assemblies and is intended to be the most challenging set of fuel parameters. Some assemblies in SFL4 are missing pins, some have pin replacements, operating parameters are changed, control rods are added and removed at different times in the burning process, and much more. The assemblies are created using outlying conditions and should therefore represent the widest range of conditions of any library. A wide range of IE, BU, and CT is also represented. Thus in analyzing this library, the goal is to see whether the algorithms are upheld in a variety of fuel conditions. Results are given in Figure 5-8. $1 \sigma$ uncertainties are shown assuming 10 minute measurements. Uncertainties are on the order of $1 \mu \mathrm{s}$.

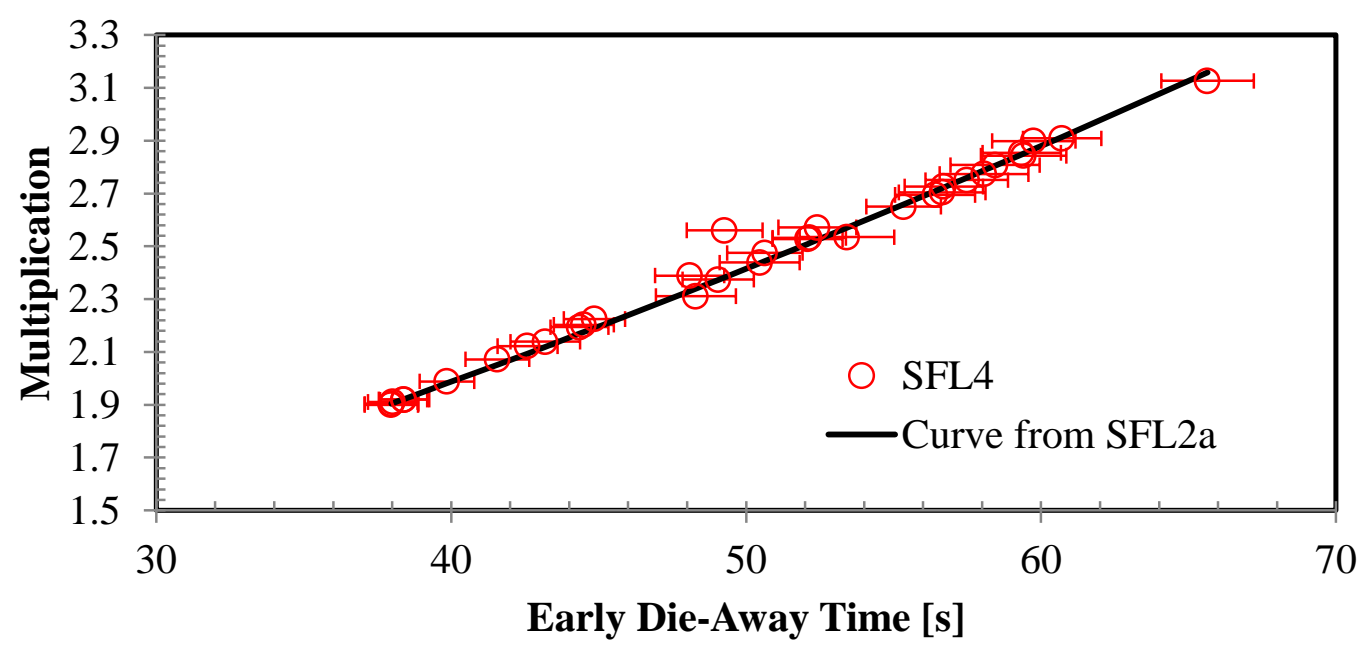

Figure 5-8. Multiplication vs. early die-away time for SFL4 assemblies. The $1 \sigma$ uncertainties on the early die-away time are given. Uncertainties on the net multiplication values are smaller than the markers used.

Applying the curve from SFL2a to the SFL4 assemblies produces a nearly perfect recreation of assembly multiplication with only a $1.5 \%$ standard deviation. The assemblies that stray most from the prediction curve are those with natural uranium fuel pins replaced, which is a very challenging scenario, as seen in Section 5.3. The fact that the SFL2a curve can accurately predict multiplication in the highly varied SFL4 assemblies indicates that the method is very robust and should be able to be applied to fuel from different reactors that have been subject to different burning conditions. 


\subsection{Spent Nuclear Fuel Assembly Plutonium Mass Calculation}

Determining the total plutonium mass in a spent fuel assembly is one of the primary goals of the NGSI spent fuel project. Motivations for quantifying Pu include strengthening the capabilities of the IAEA to safeguard facilities, quantifying shipper/receiver differences, and determining input accountability and burnup credit at reprocessing facilities [73]. Because the content may vary significantly both axially and radially, destructive assay techniques in which a small sample of a fuel rod is analyzed will not be accurate. Nondestructive assay provides an opportunity to utilize the total neutron signal, which is produced directly from $\mathrm{Pu}$ isotopes through the burning process, and correct it for multiplication to determine Pu mass in the complete assembly.

\subsubsection{DDSI Methodology}

Applying the concepts developed through the DDA work [33], it can be shown that $m_{P u}$ can be determined in simulation space using the DDSI instrument with a similarly high precision. The method has been tested against 44 simulated SFAs from Spent Fuel Library 2a (SFL2a), 37 various assemblies in SFL4, and 44 assemblies in SFL6 (in Section 5.3). The results were qualitatively as well as quantitatively nearly identical to those obtained previously for the Differential Die-Away instrument thus demonstrating the robustness of the method.

As discussed, the total neutron emission rate corrected for multiplication can be used to determine Pu mass. The multiplication reflects the competition between the fissile content and the amount and composition of the neutron absorbers (fission products and minor actinides), and is implicitly defined by the SFA characteristic parameters such as IE, BU, and CT. The total neutron emission, however, reflects mass content of major spontaneous fission isotopes in the assembly including ${ }^{242} \mathrm{Cm},{ }^{244} \mathrm{Cm}$, and ${ }^{240} \mathrm{Pu}$ as well as the main contributors to neutron production via $(\alpha, n)$

reaction (e.g. $\left.{ }^{241} \mathrm{Am}\right)$. Since the majority of these isotopes are produced by processes that include one or more neutron captures on individual Pu isotopes, their quantity, and thus the neutron emission rate, is closely correlated to the total content of elemental plutonium (i.e., $m_{p u}$ ) [74]. A visual representation of various production modes of transuranics in the process of nuclear burning can be found in chapter 4, Figure 3-2.

In this work, eq.(16) from [33] is utilized relating the neutron emission rate (NE), multiplication $(M)$, and total plutonium mass $\left(m_{p u}\right)$ 


$$
m_{p u}=a(C T)\left(\frac{M+2 c}{M+c}\right)\left[\left(\frac{M}{M+2 c}\right) N E\right]^{b(C T)}
$$

with $a, b$, and $c$ being instrument dependent calibration parameters, which in the case of $a$ and $b$ may also be functions of CT. However, as the authors of [33] modified the equation above to include quantities measurable by the DDA instrument (eq.(20) in [33]), we modified the equation to include only quantities directly measurable by the DDSI instrument. Previously [9] it was reported that the die-away time of the early time domain (4-52 $\mu \mathrm{s})$ of the RAD of the DDSI instruments response is quadratically related with the SFA multiplication:

$$
M=a_{1} \tau_{e}^{2}+a_{2} \tau_{e}+a_{3}
$$

where $a_{1}, a_{2}$, and $a_{3}$ are the fitting (i.e., calibration) parameters. In the case of the DDSI instrument the singles rate $(S)$ is identical to the passive neutron count rate used in [33], and through a simple relation with $M$ it is then directly representative of the NE term in eq.(11):

$$
N E=S /(M \times \varepsilon)
$$

where $\varepsilon$ is the system efficiency. Assuming that efficiency is absorbed into the calibration parameters, the eq.(11), i.e., the expression for determination of $m_{p u}$, can be rewritten using only quantities directly measurable by the DDSI instrument as follows [34]:

$$
m_{p u}=d(C T)\left(\frac{\tau_{e}+g}{\tau_{e}+h}\right)\left(\frac{S}{\tau_{e}+g}\right)^{f(C T)}
$$

where $d, f, g$ and $h$ are the calibration parameters which depend on the instrument design and in case of $d$ and $f$ also on CT. To solve for $\mathrm{d}$ and $\mathrm{f}$ and find the optimal values of $\mathrm{g}$ and $\mathrm{h}$, equation (13) is rearranged in the form of a power function with $x=\frac{s}{\tau_{e}+g}$ and $y=\frac{m_{p u}\left(\tau_{e}+h\right)}{\tau_{e}+g}$. The calibration constants $\mathrm{g}$ and $\mathrm{h}$ are initially the same values determined in the DDA work [33], then are lowered and raised iteratively to make a smooth power function for data from a single cooling time, as shown in Figure 5-9. 

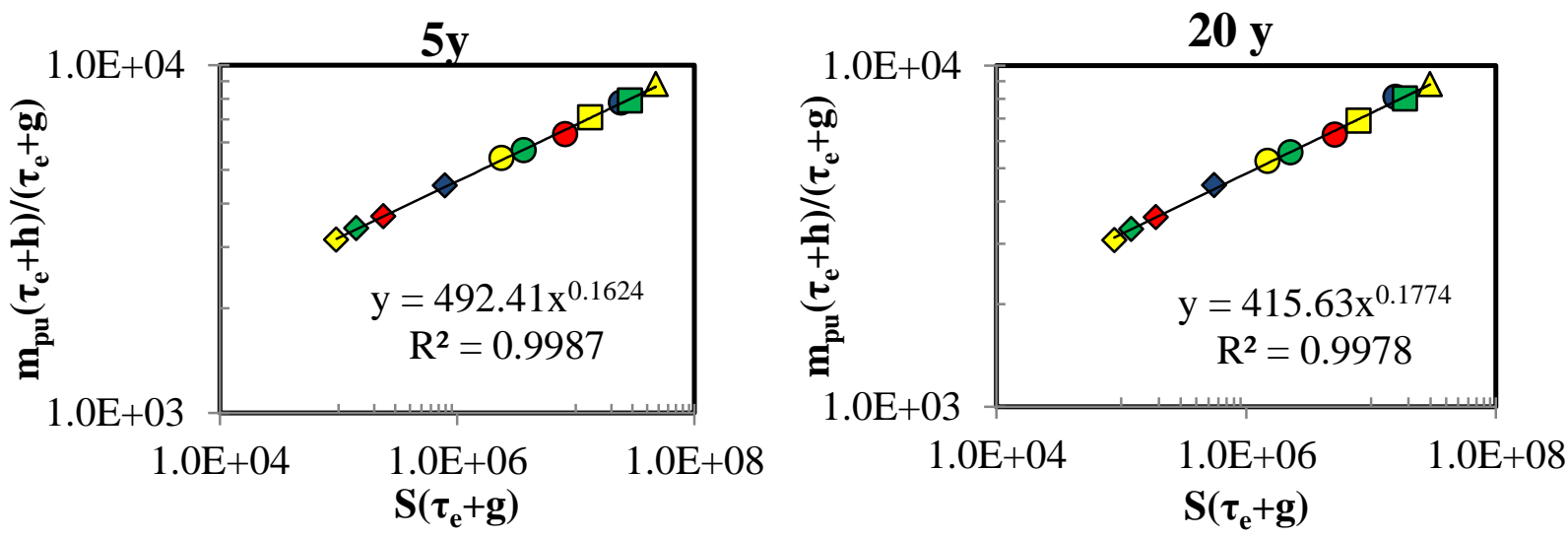

$$
\begin{gathered}
\square \mathrm{IE}=2 \% \square \mathrm{IE}=3 \% \square \mathrm{IE}=4 \% \square \mathrm{IE}=5 \% \\
\diamond \mathrm{BU}=15 \mathrm{GWd} / \mathrm{tU} \bigcirc \mathrm{BU}=30 \mathrm{GWd} / \mathrm{tU} \square \mathrm{BU}=45 \mathrm{GWd} / \mathrm{tU} \triangle \mathrm{BU}=60 \mathrm{GWd} / \mathrm{tU}
\end{gathered}
$$
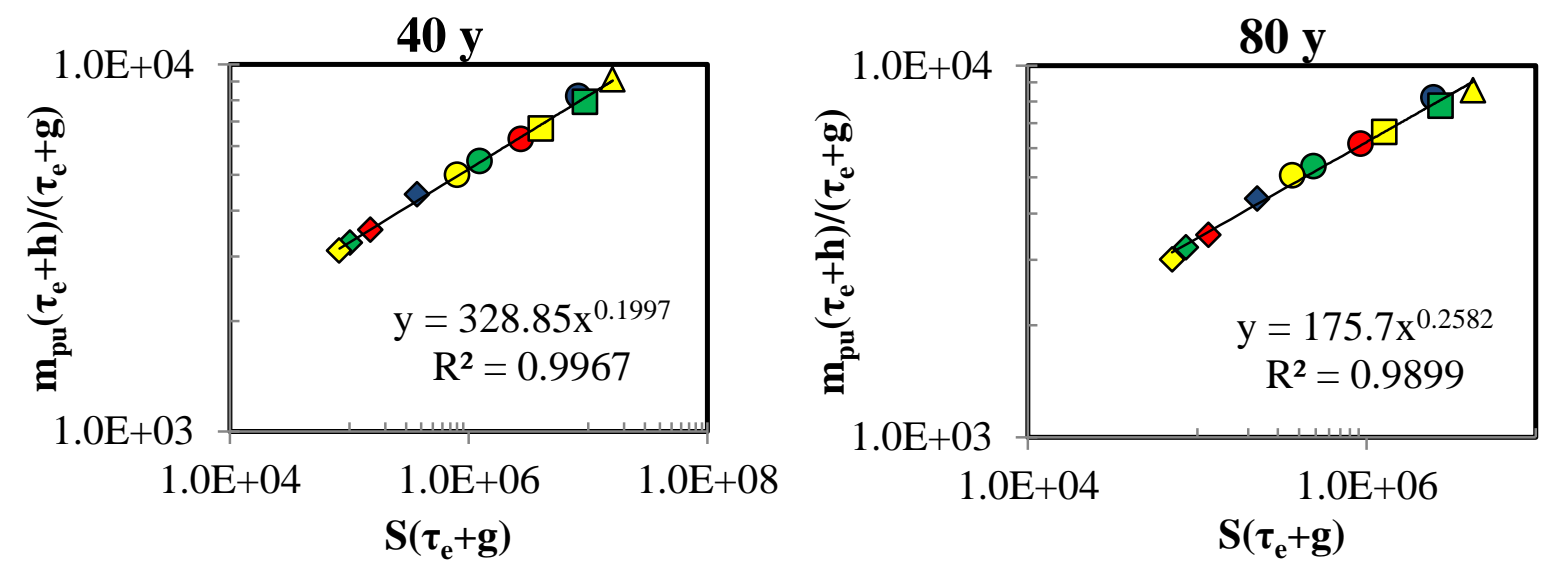

Figure 5-9. Plots for each cooling time to determine calibration parameters.

The constant $d(C T)$ and $f(C T)$ values are determined from the power function fits with $y=d(C T) x^{-f(C T)}$. If more assembly cooling times were simulated than used here, a calibration curve could be created to determine $d(C T)$ and $f(C T)$ for assemblies with cooling times that are not equal to those in the simulated assemblies.

\subsubsection{Results from Various Spent Fuel Libraries}

\section{(i) SFL2a}

All simulations were conducted with MCNP [58]. Coincidence counting data are simulated in the form of RADs which are discussed in detail in chapter 2. In total, the assay of 44 pressurized water reactor assemblies was simulated from SFL2a with IE varying from 2 to 5\%, BU varying from 15 to $60 \mathrm{GWd} / \mathrm{tU}$, and CT varying from 5 to 80 years. The SFAs are $17 \times 17$ Westinghouse type with one 
radial region tracked through a 1/8 core using Monteburns to track isotopic changes through the burning process [56]. While the SFLs are designed to describe the complex isotopic composition of SFAs as realistically as possible, various approximations are still being made during their development by assuming, for example, homogenous axial BU profiles and disregarding fuel expansion and rim effects, among others.

The two panels of Figure 5-10 display results of the simulations in terms of the detected singles rate ( $S$; left panel) and the measured die-away time $\left(\tau_{e}\right)$ in the early time domain (4-52 $\mu \mathrm{s}$; right panel). Both panels thus demonstrate the complex interplay of IE, BU and CT on the measurable signals with respect to the true $\mathrm{Pu}$ content, yet the correction introduced in eq. (6) removes most of these dependences without an explicit knowledge of IE, BU and possibly even CT, as can be seen in the left panel of Figure 5-10. The right panel of Figure 5-11 then displays the relative differences between the $m_{p u}$ determined from eq. (6) and the true $m_{p u}$ as known from the material definition of individual SFAs. The numerical summary of data displayed in Figure 5-11 is listed in Table 5-IV. The RMS error of the entire data set is $2.1 \%$, although the RMS error of the subset of data with CT $\leq$ $40 \mathrm{y}$ is only $1.5 \%$, while the RMS error of the complementary set of data with CT $=80 \mathrm{y}$ is $3.4 \%$. 

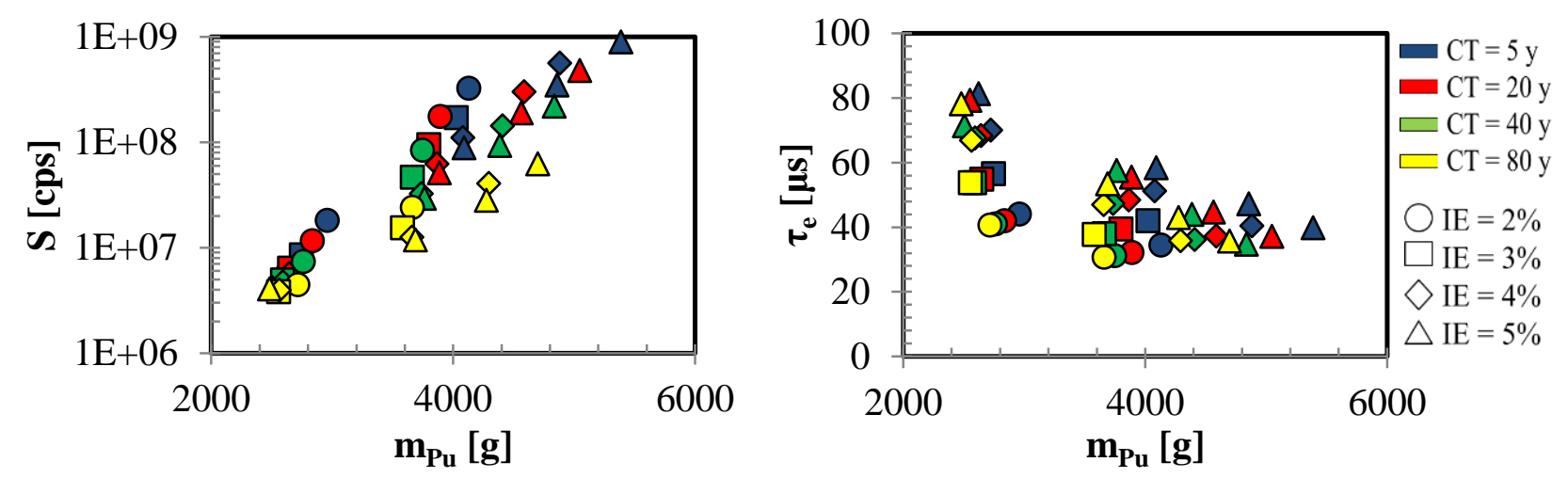

Figure 5-10. Results of the simulations of the detected singles rate $S$ (left) and $\tau_{\mathrm{e}}$ (right) for 44 SFAs from SFL2a as measured by the DDSI instrument.
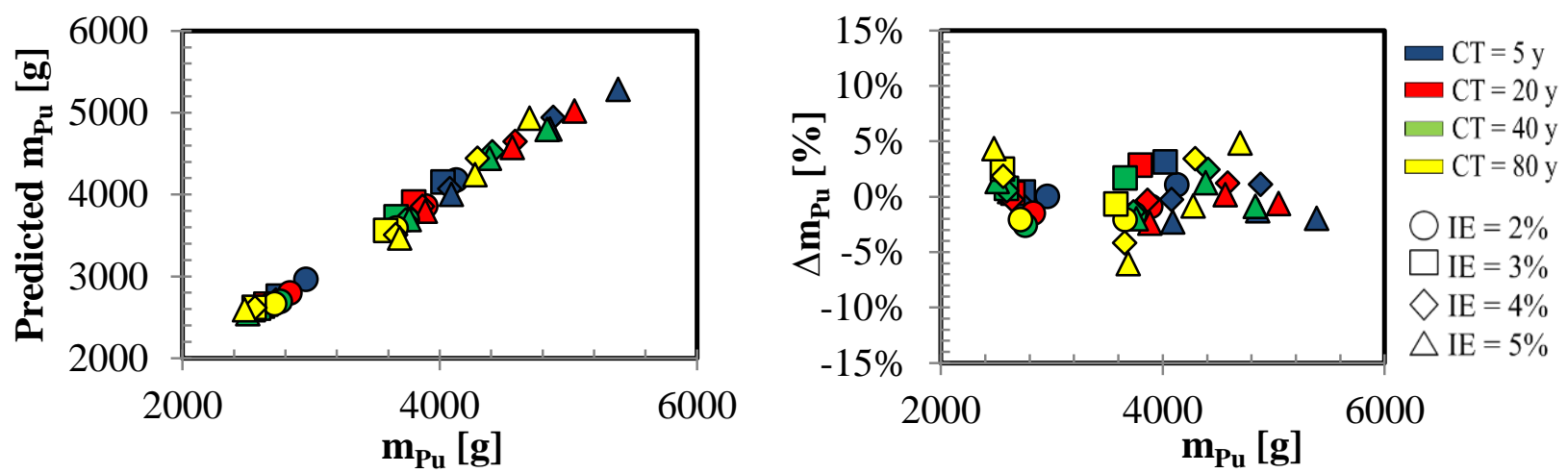

Figure 5-11. Comparison of the absolute values of the $m_{P u}$ determined from eq. (6) and the true $m_{P u}$ in the SFA (left) and the relative differences between the determined $m_{P u}$ and the true values (right). 
Table 5-IV. Summary of true and determined $\mathrm{m}_{\mathrm{Pu}}$ for 44 SFAs from SFL2a divided into subsets by different CT values.

\begin{tabular}{|c|c|c|c|c|c|c|c|c|c|}
\hline $\begin{array}{c}\text { BU } \\
{[\mathbf{G W d}} \\
/ \mathbf{t U}]\end{array}$ & $\begin{array}{c}\mathrm{IE} \\
{[\%]}\end{array}$ & $\begin{array}{c}\text { true } \\
m_{P u} \\
{[\mathrm{~g}]}\end{array}$ & $\begin{array}{c}\text { det. } \\
m_{P u} \\
{[\mathrm{~g}]}\end{array}$ & $\begin{array}{c}\Delta m_{P u} \\
{[\%]}\end{array}$ & $\begin{array}{c}\text { BU } \\
{[\mathbf{G W d}} \\
/ \mathbf{t U}]\end{array}$ & $\begin{array}{c}\text { IE } \\
{[\%]}\end{array}$ & $\begin{array}{c}\text { true } \\
m_{P u} \\
{[\mathrm{~g}]}\end{array}$ & $\begin{array}{c}\text { det. } \\
m_{P u} \\
{[\mathrm{~g}]}\end{array}$ & $\begin{array}{c}\Delta \boldsymbol{m}_{P u} \\
{[\%]}\end{array}$ \\
\hline \multicolumn{5}{|c|}{$\mathrm{CT}=5 \mathrm{y}$} & \multicolumn{5}{|c|}{$C T=20 y$} \\
\hline 15 & 2 & 2961.5 & 2961.6 & $0.00 \%$ & 15 & 2 & 2836.6 & 2793.5 & $-1.52 \%$ \\
\hline 15 & 3 & 2746.5 & 2757.9 & $0.42 \%$ & 15 & 3 & 2649.8 & 2657.8 & $0.30 \%$ \\
\hline 15 & 4 & 2725.4 & 2709.2 & $-0.59 \%$ & 15 & 4 & 2644.0 & 2636.2 & $-0.29 \%$ \\
\hline 15 & 5 & 2622.6 & 2634.5 & $0.45 \%$ & 15 & 5 & 2551.4 & 2590.5 & $1.53 \%$ \\
\hline 30 & 2 & 4130.1 & 4174.2 & $1.07 \%$ & 30 & 2 & 3892.1 & 3857.9 & $-0.88 \%$ \\
\hline 30 & 3 & 4025.3 & 4150.7 & $3.11 \%$ & 30 & 3 & 3799.4 & 3906.7 & $2.83 \%$ \\
\hline 30 & 4 & 4080.6 & 4068.6 & $-0.29 \%$ & 30 & 4 & 3866.3 & 3852.1 & $-0.37 \%$ \\
\hline 30 & 5 & 4092.9 & 3998.9 & $-2.30 \%$ & 30 & 5 & 3888.2 & 3794.2 & $-2.42 \%$ \\
\hline 45 & 4 & 4883.8 & 4936.7 & $1.08 \%$ & 45 & 4 & 4588.9 & 4643.4 & $1.19 \%$ \\
\hline 45 & 5 & 4858.1 & 4796.1 & $-1.28 \%$ & 45 & 5 & 4565.4 & 4573.2 & $0.17 \%$ \\
\hline 60 & 5 & 5388.0 & 5280.7 & $-1.99 \%$ & 60 & 5 & 5047.6 & 5015.4 & $-0.64 \%$ \\
\hline \multicolumn{4}{|c|}{ Standard Deviation: } & $1.5 \%$ & \multicolumn{4}{|c|}{ Standard Deviation: } & $1.4 \%$ \\
\hline \multicolumn{5}{|c|}{$C T=40 y$} & \multicolumn{5}{|c|}{$\mathrm{CT}=80 \mathrm{y}$} \\
\hline 15 & 2 & 2761.9 & 2690.1 & $-2.60 \%$ & 15 & 2 & 2717.7 & 2660.6 & $-2.10 \%$ \\
\hline 15 & 3 & 2591.9 & 2610.9 & $0.73 \%$ & 15 & 3 & 2557.3 & 2620.4 & $2.47 \%$ \\
\hline 15 & 4 & 2595.0 & 2608.6 & $0.53 \%$ & 15 & 4 & 2565.4 & 2611.8 & $1.81 \%$ \\
\hline 15 & 5 & 2508.6 & 2542.3 & $1.34 \%$ & 15 & 5 & 2482.4 & 2589.8 & $4.33 \%$ \\
\hline 30 & 2 & 3748.9 & 3688.2 & $-1.62 \%$ & 30 & 2 & 3662.0 & 3586.1 & $-2.07 \%$ \\
\hline 30 & 3 & 3663.3 & 3724.5 & $1.67 \%$ & 30 & 3 & 3580.8 & 3556.4 & $-0.68 \%$ \\
\hline 30 & 4 & 3737.2 & 3685.1 & $-1.40 \%$ & 30 & 4 & 3659.3 & 3506.7 & $-4.17 \%$ \\
\hline 30 & 5 & 3764.7 & 3688.9 & $-2.01 \%$ & 30 & 5 & 3689.8 & 3465.8 & $-6.07 \%$ \\
\hline 45 & 4 & 4408.9 & 4516.0 & $2.43 \%$ & 45 & 4 & 4294.2 & 4440.1 & $3.40 \%$ \\
\hline 45 & 5 & 4387.3 & 4441.5 & $1.24 \%$ & 45 & 5 & 4275.9 & 4238.5 & $-0.87 \%$ \\
\hline 60 & 5 & 4838.0 & 4794.2 & $-0.91 \%$ & 60 & 5 & 4700.3 & 4927.0 & $4.82 \%$ \\
\hline \multicolumn{4}{|c|}{ Standard Deviation: } & $1.6 \%$ & \multicolumn{4}{|c|}{ Standard Deviation: } & $3.4 \%$ \\
\hline
\end{tabular}

As in [4], since only 4 different cooling times were investigated, the dependence of $d(\mathrm{CT})$ and $f(\mathrm{CT})$ indicated in Figure 5-12 should be considered with limitations, however the trend seems to be rather smooth raising the possibility of a straight forward calibration with more data. Comparing these 
trends to results in [33] it is worth noting that the results in this work trend consistently in a linear fashion while the previous results do not, as shown in Figure 5-12. However, the CT of $1 \mathrm{y}$ that breaks the linear trend in the DDA results was not investigated in this work, therefore based on the range of CT investigated in both papers, it can still be concluded that the CT dependence of the fitting parameters of eq. (6) is qualitatively very similar.
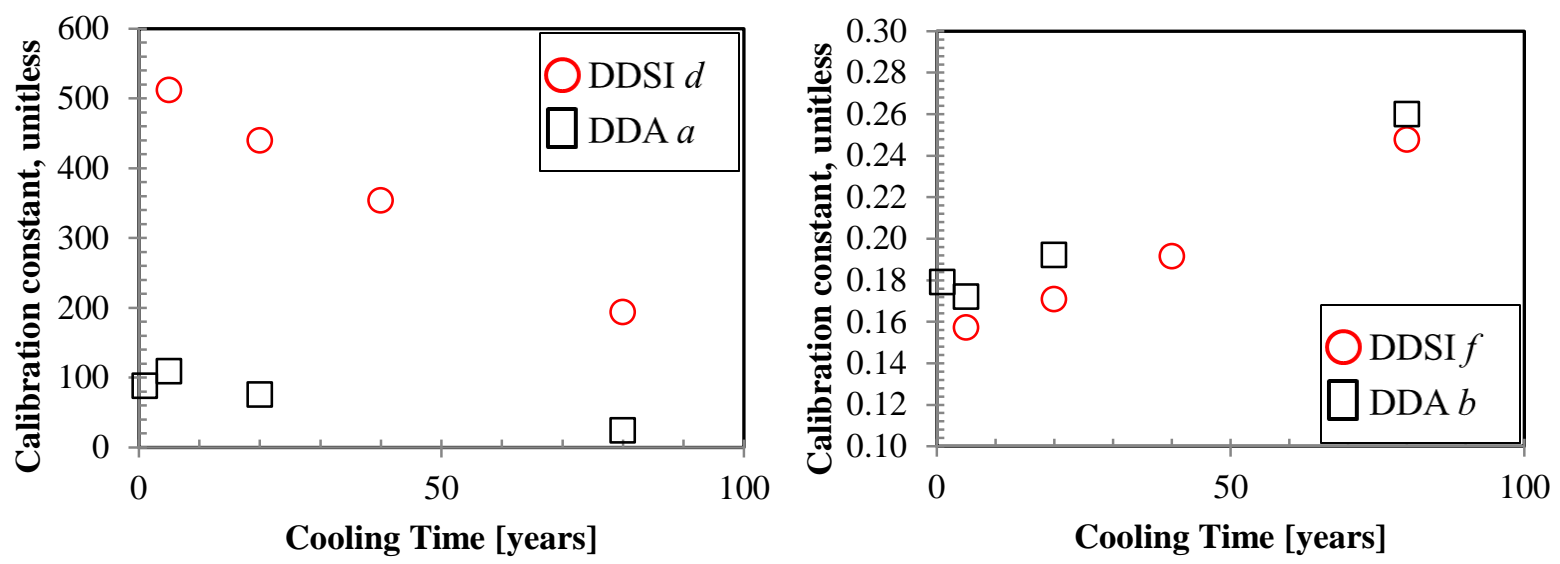

Figure 5-12. Values of the fitting parameters $d$ and $f$ from eq. (6) and a and $b$ from eq.(16) from [33], as a function of CT.

(ii) Results from SFL3

SFL3 is described in detail in Sections 3.2 and 5.1.5. In applying the multiplication determination algorithm to SFL3 data, it was determined that though the trend was independent of the assembly parameters varied in SFL3, a break in the data existed between the two sets with different burnup levels. The most likely reason for this break is that burnups were simulated differently in the ORNL burnup code, rather than taking an assembly, burning it to $30 \mathrm{GWd} / \mathrm{tU}$ and recording the results, then continuing to burn it to $45 \mathrm{GWd} / \mathrm{tU}$. This issue showed itself again when the total Pu algorithm was applied to SFL3 data. The changing operating parameters do not affect the results, however there is a break between the different burnups. The 5 y results for $30 \mathrm{GWd} / \mathrm{tU}$ and $45 \mathrm{GWd} / \mathrm{tU}$ are given in Figure 5-14. 


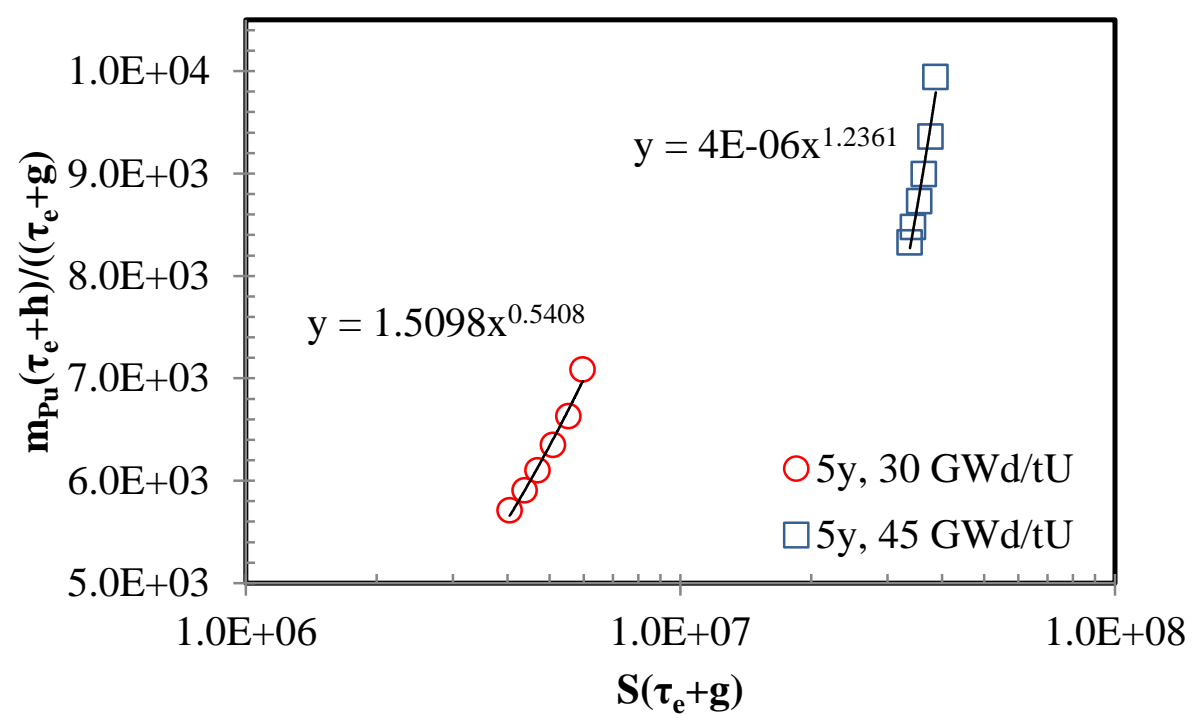

Figure 5-13. Pu determination algorithm applied to SFL3 data to determine calibration constants. Different constants are needed for different burnups, as demonstrated here.

(iii) Results from SFL4

Recall that SFL4 contains "mystery" assemblies with a wide variety of operating conditions and fuel parameters. The SFL4 assemblies were divided up by cooling time and the calibration curve from SFL2a was applied to the data. The result is given in Figure 5-14. The plot shows the percent difference between determined Pu mass and actual Pu mass in each assembly as a function of actual Pu mass for the different cooling times in SFL4.

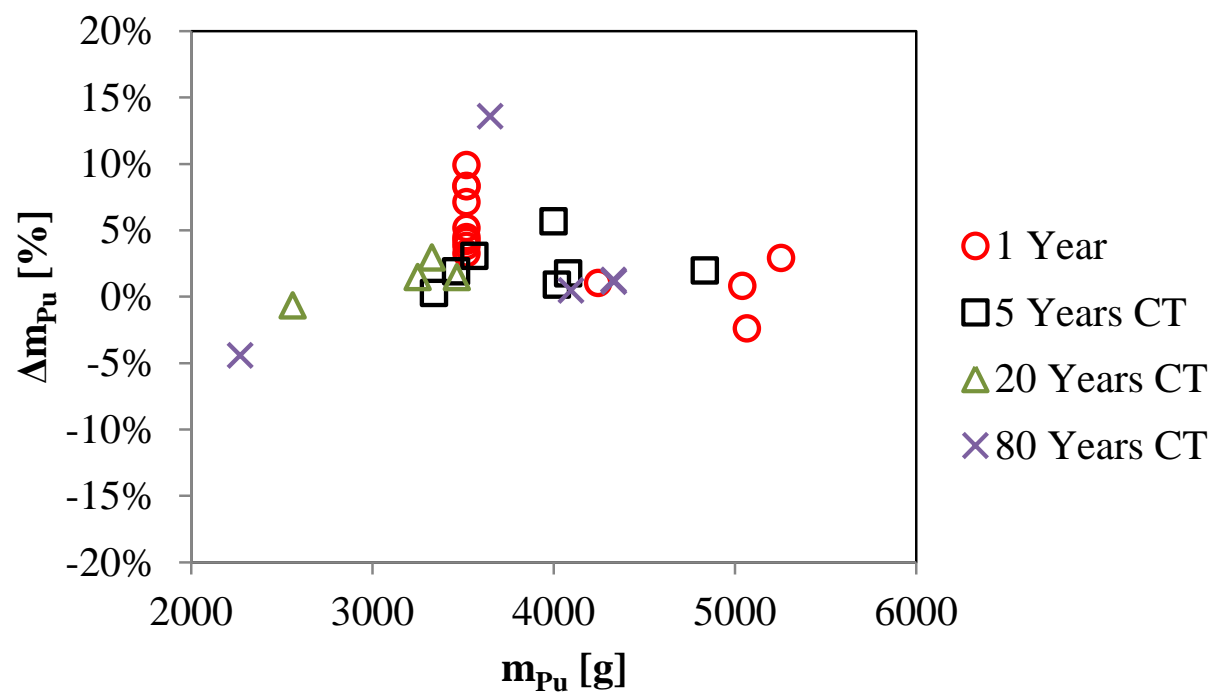

Figure 5-14. Difference in predicted and actual Pu mass as a function of Pu mass in SFL4 assemblies. 
Note that the majority of points are near $0 \%$, indicating good agreement between predicted and actual plutonium mass. A series of 1 year cooled assemblies have the same Pu mass because they are all identical assemblies but with different pins removed and replaced with different dummy materials, therefore they have the same Pu mass but slightly varying multiplication values because of the varying replacement pins, resulting in different $\mathrm{Pu}$ mass predictions. The method does, on average, a very good job of predicting Pu mass in SFL4 assemblies despite the calibration curve coming from a different burnup code than most assemblies and having very different operating parameters. The standard deviation is $4.79 \%$. Considering the uncertainty in Pu mass in simulated assemblies, this method does well in predicting Pu mass.

\subsection{Missing Pin Detection}

One of the primary goals of the National Nuclear Security Administration's NGSI-SF project is to utilize new nondestructive assay techniques for detecting the illicit diversion or replacement of pins in spent fuel assemblies (SFAs) [13]. For example, a 2010 IAEA publication on safeguards for encapsulation plants explicitly states that the SFAs should be "verified by a partial defect test," if possible [75]. Neutron coincidence counting (NCC) has been used for several decades to detect the presence of materials that exhibit spontaneous and induced fission [1]. With the development of the DDSI [3], NCC has also been investigated within NGSI-SF as a technique for characterizing spent nuclear fuel [72].

Throughout its history the NGSI-SF project has investigated the use of several NDA instruments for missing pin detection $[76,77,78]$. The primary approach has been utilization of a reference assembly or set of assemblies for comparison with the one assayed. This approach simply uses the signal from an NDA instrument to measure assemblies with suspected missing pins and compares the result with that of a complete assembly. This approach is explored in section 8.3. Although potentially very powerful, this approach presents a significant challenge in real measurement scenarios where a reference signal for already-existing spent fuel is nonexistent and/or may be very difficult to reliably obtain for future SFAs. Therefore, a self-consistent method of pin diversion detection is also investigated here in section 8.4. The first self-consistent method based on neutron detection that was investigated within NGSI-SF utilized the Differential Die-Away (DDA) instrument [78] and seemed 
to be able to identify certain scenarios of removal or substitution of pins in the center of the SFA in a single assay measurement by considering ratios of signal die-away times observed by different individual detectors. This and other alternate methods of pin diversion detection are discussed further in section 8.2. Another self-consistent, and perhaps complementary, neutron detection-based approach is proposed which is adoptable by any instrument that can measure multiplication and passive neutron emission in cases in which there is certain knowledge of the SFA cooling time. This will be discussed in the following sections.

\subsubsection{Simulated Diversion Scenarios}

Through the NGSI-SF effort, a series of spent fuel libraries (SFLs) have been produced [53], representing a wide variety of combinations of assembly parameters, including burnup (BU), initial enrichment (IE), cooling time (CT), moderator density, shuffling patterns, and many others. For this work, SFL2a and SFL6 libraries are considered. SFL2a contains "base" assemblies with varying IE, BU, CT, and standard shuffling patterns, and it is used in two different ways: (1) It contains the set of assemblies on which the calibration is performed and is applied to the diverted assemblies. (2) It provides the "operator declaration" that would be the reference for comparison with the results from a real measurement. SFL6 contains the same assemblies as SFL2a, but it is limited to assemblies cooled for 5 years, and 40 fuel pins in each assembly have been diverted and replaced with either stainless steel or natural uranium. Table 5-V shows the BU and IE combinations considered. While a wide range of SFA parameters is covered, only combinations considered "standard" and realistic are included. For example, assemblies with IE lower than $5 \%$ are typically not burned to $60 \mathrm{GWd} / \mathrm{tU}$, and thus are not included.

Table 5-V. BU/IE Combinations in SFL6.

\begin{tabular}{|c|c|c|c|c|}
\hline & \multicolumn{4}{|c|}{$\mathrm{BU}[\mathrm{GWd} / \mathrm{tU}]$} \\
\hline & 15 & 30 & 45 & 60 \\
\hline IE [\%] & $2,3,4,5$ & $2,3,4,5$ & 4,5 & 5 \\
\hline
\end{tabular}

The diversion scenarios considered in this work represent two different and rather extreme pin diversion arrangements (shown in Figure 5-15) and two very plausible material replacements: NU and SS. It should be noted that the dispersed diversion scenario that was studied could be identified 
by appropriate gamma-ray measurement methods (such as gamma emission tomography) because several of the pins were removed from the second outer row, which is directly visible to a collimated gamma detector. However, gamma detection is not considered in this work, and the dispersed diversion scenario is chosen to probe the maximum reasonably possible variation in pin diversion to test the limit of the method based on total Pu determination by neutron detection methods.

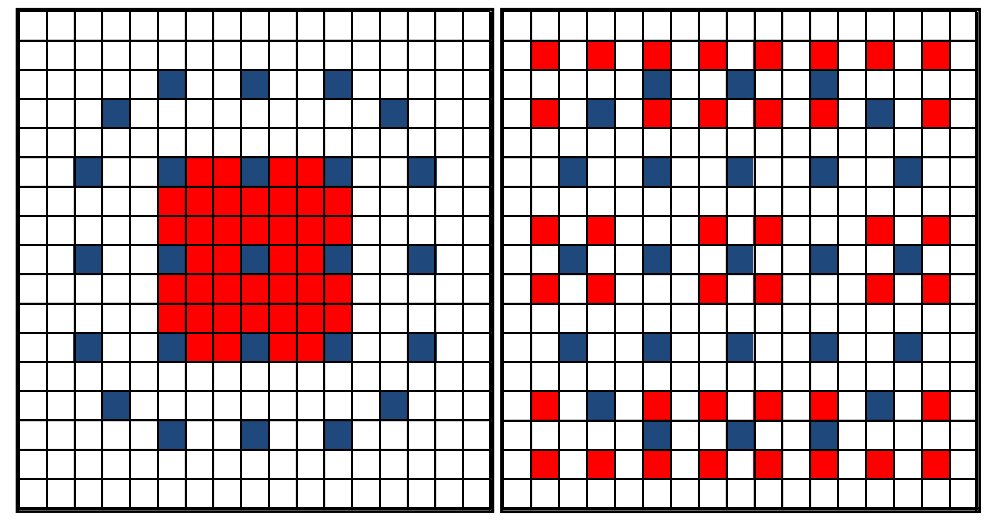

Figure 5-15. Diversion scenarios: center (left) and dispersed (right). Both scenarios have 40 pins (red) that have been replaced by pins with either SS or NU.

In order to detect a diversion, observable features of the assemblies must be identified that are sufficiently different from their expected values in order for those features to be flagged as suspicious. The aforementioned and difficult to apply reference assembly approach uses a variety of features to identify abnormal assemblies. The self-consistent approach focuses on the total $\mathrm{Pu}$ content, as explicitly or implicitly declared by the operator.

The Pu quantity diverted in each assembly is slightly different for each diversion scenario and BU/IE combination due to pin-to-pin differences in isotopic compositions. Mass fractions multiplied by pin mass from MCNP assembly definitions were used to determine the Pu mass removed in each case. Detailed information on plutonium removal can be found in [79]. The reference assembly approach is described in the following section.

\subsubsection{Reference Assembly Approach}

The Rossi-alpha distributions with pins diverted and replaced were then compared to those with complete assemblies [80]. Figure 5-16 is a standard $45 \mathrm{GWd} / \mathrm{tU}, 4 \%, 5$ y cooled assembly with no diversions. 


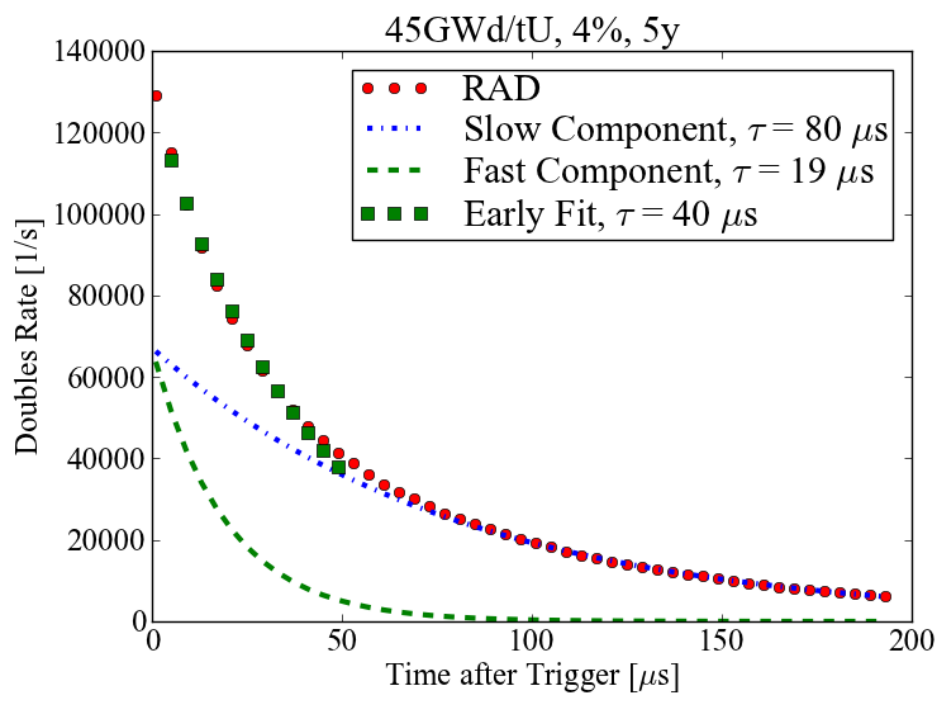

Figure 5-16. Standard $45 \mathrm{GWd} / \mathrm{tU}, 4 \%$, 5 year cooled assembly with all pins intact. Early dieaway time $=40 \mu \mathrm{s}$, fast die-away time $=19.0 \mu \mathrm{s}$, and the slow die-away time $=80 \mu \mathrm{s}$.

The first two diversions which are from different parts of the assembly but both have natural uranium replacement pins, are shown in Figure 5-17.
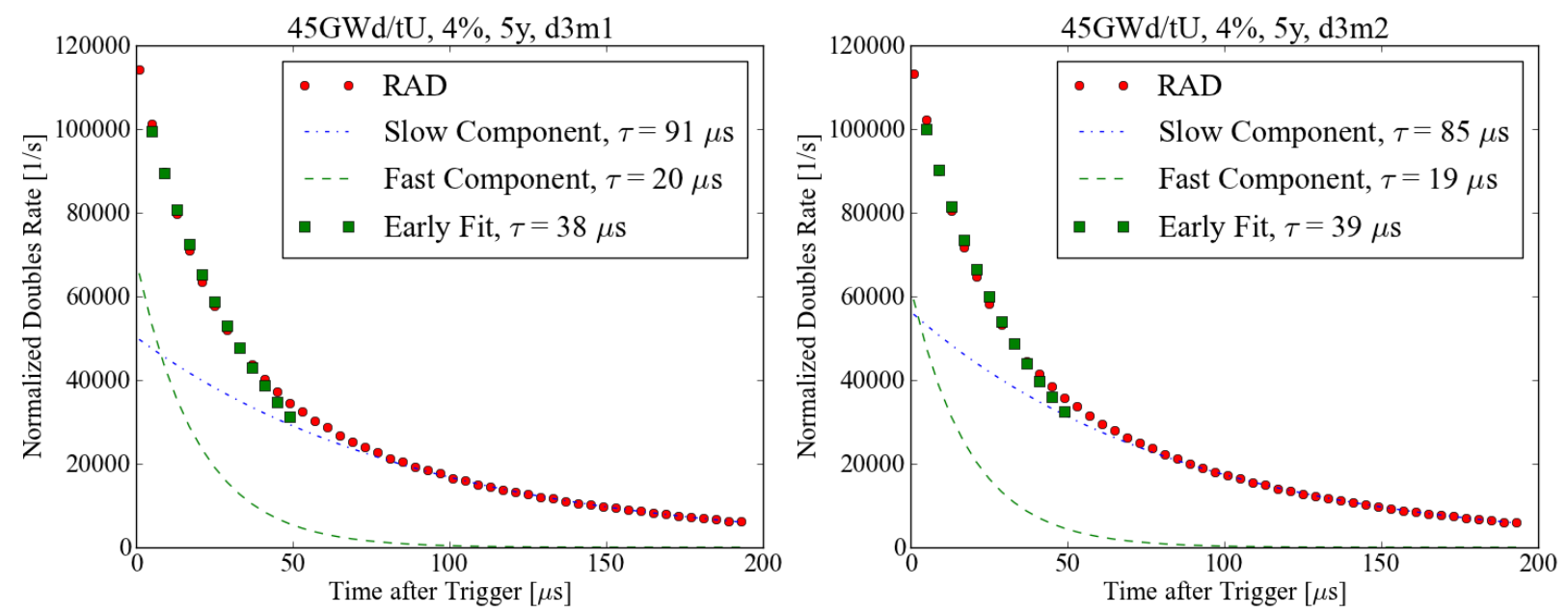

Figure 5-17. 45, 4, 5 assembly with 40 pins replaced with $\mathrm{NU}$. Center diversion $(l e f t)$ and dispersed diversion (right).

The magnitude of the slow component is noticeably suppressed in the center diversion scenario, however the slow die-away time is longer in both NU replacements. Early die-away times drop slightly reflecting the decrease in multiplication. The fast die-away times remain roughly constant as expected because the detector geometry does not change. 
The SS replacements are shown in Figure 5-18.
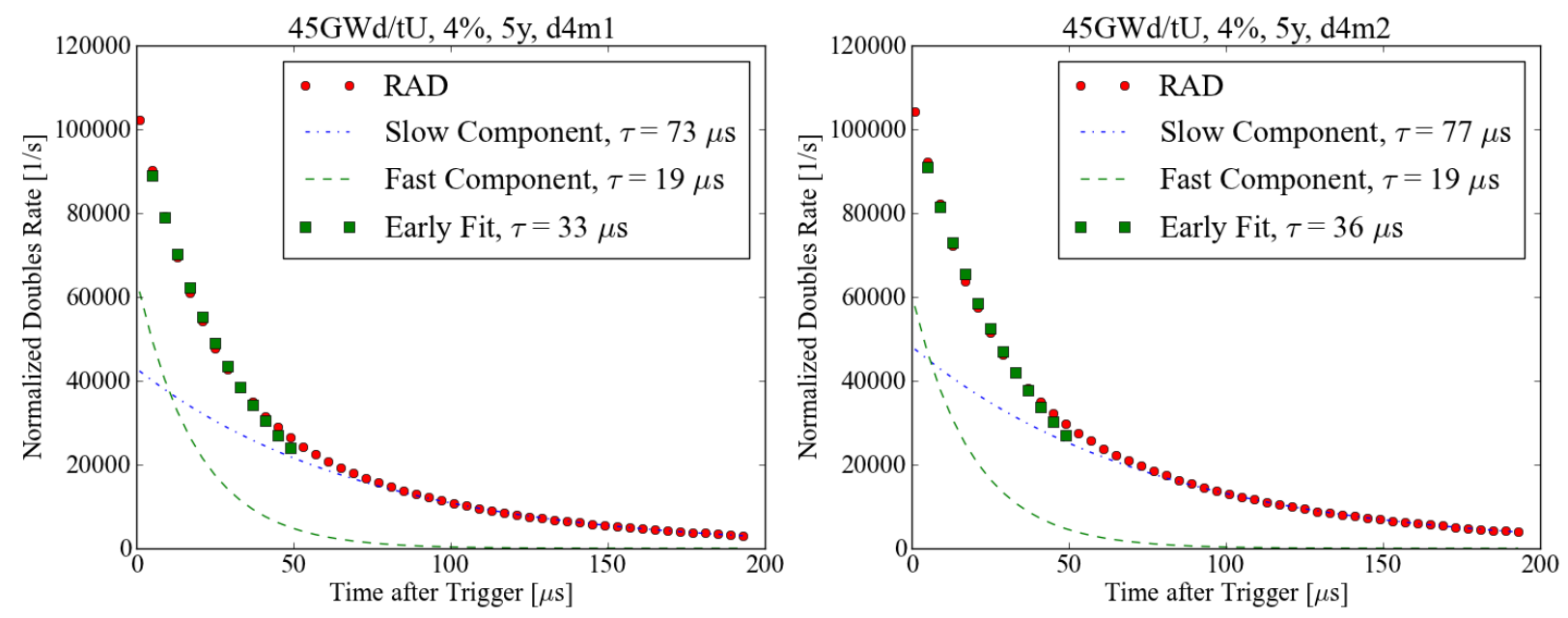

\section{Figure 5-18. 45, 4, 5 assembly with 40 pins replaced with stainless steel. Center diversion (left) and dispersed diversion (right).}

The magnitude of the slow component was considerably smaller in both cases, and the die-away time was faster in both cases as well. The early die-away times both dropped much more because there is no opportunity for added multiplication from SS pins. The fast die-away times again remained constant. Some figures from the RADs are compared in Table 5-VI. $1 \sigma$ uncertainty values are quoted assuming a 10 minute measurement. 
Table 5-VI. Comparison of statistics from 45GWd/tU, 4\%, 5y RADs

\begin{tabular}{ccccccc}
\hline Assembly & Early $\tau$ & Fast $\tau$ & Slow $\tau$ & $\begin{array}{c}\text { Fast/Slow } \\
\text { magnitude }\end{array}$ & Singles & Multiplication \\
\hline Normal & 40.4 & 19.4 & 81.2 & 1.00 & $22,313,421$ & 2.00 \\
& $( \pm 0.9)$ & $( \pm 0.1)$ & $( \pm 0.5)$ & $( \pm 0.01)$ & $( \pm 193)$ & \\
NU, Cen. & 40.0 & 19.4 & 91.7 & 1.40 & $5,263,198$ & 1.95 \\
& $( \pm 1.0)$ & $( \pm 0.1)$ & $( \pm 0.7)$ & $( \pm 0.02)$ & $( \pm 94)$ & \\
NU, Dis. & 41.0 & 18.5 & 85.3 & 1.13 & $2,252,874$ & 1.97 \\
& $( \pm 1.1)$ & $( \pm 0.2)$ & $( \pm 0.4)$ & $( \pm 0.01)$ & $( \pm 94)$ & \\
SS, Cen. & 35.0 & 19.0 & 73.3 & 1.56 & $4,927,316$ & 1.69 \\
& $( \pm 0.8)$ & $( \pm 0.2)$ & $( \pm 0.5)$ & $( \pm 0.02)$ & $( \pm 91)$ & \\
SS, Dis. & 37.9 & 19.0 & 77.1 & 1.29 & $5,004,940$ & 1.82 \\
& $( \pm 0.9)$ & $( \pm 0.2)$ & $( \pm 0.4)$ & $( \pm 0.02)$ & $( \pm 91)$ & \\
\hline
\end{tabular}

Table 5-VI demonstrates quantitatively the changes in RAD signals with pin diversions and replacements. Multiplication decreased when a SS replacement was used, and early die-away time reflected the multiplication decrease. The multiplication (and early die-away time) do not change with statistical significance when natural uranium is the replacement material, thus it could not be used as a standalone indication of diversion. Slow die-away time changed to become higher or lower than the reference assembly based upon pin replacement material, however it changed more than $3 \sigma$ in all cases, indicating that an abnormality would be detected regardless of whether the signal was unexpectedly high or low. The fast die-away time remained the same for all cases indicating that the fitting procedure was correct; fast die-away time reflects the instrument die-away time and thus should not change with a changing source. Fast/slow magnitude was a particularly interesting quantity that changed as much as 50\% in the case of the center replacement with SS. Even in the closest case of NU, dispersed, the value changed by over $10 \sigma$. A plot of percent difference between diverted assembly fast/slow magnitude and complete assembly fast/slow magnitude is given in Figure 5-19. 3 $\sigma$ error bars are given assuming a 10 minute count time. Additional uncertainty will of course be introduced in experiment and therefore these should not be taken as absolute uncertainties, but from the large change in certain indicators it can be deduced that the reference assembly 
approach using RAD indicators could detect pin diversions. A strategic combination of these indicators would be the best way to identify diversions. Spent fuel experiments conducted with diversion scenarios will be the best way to determine an optimal combination of indicators.

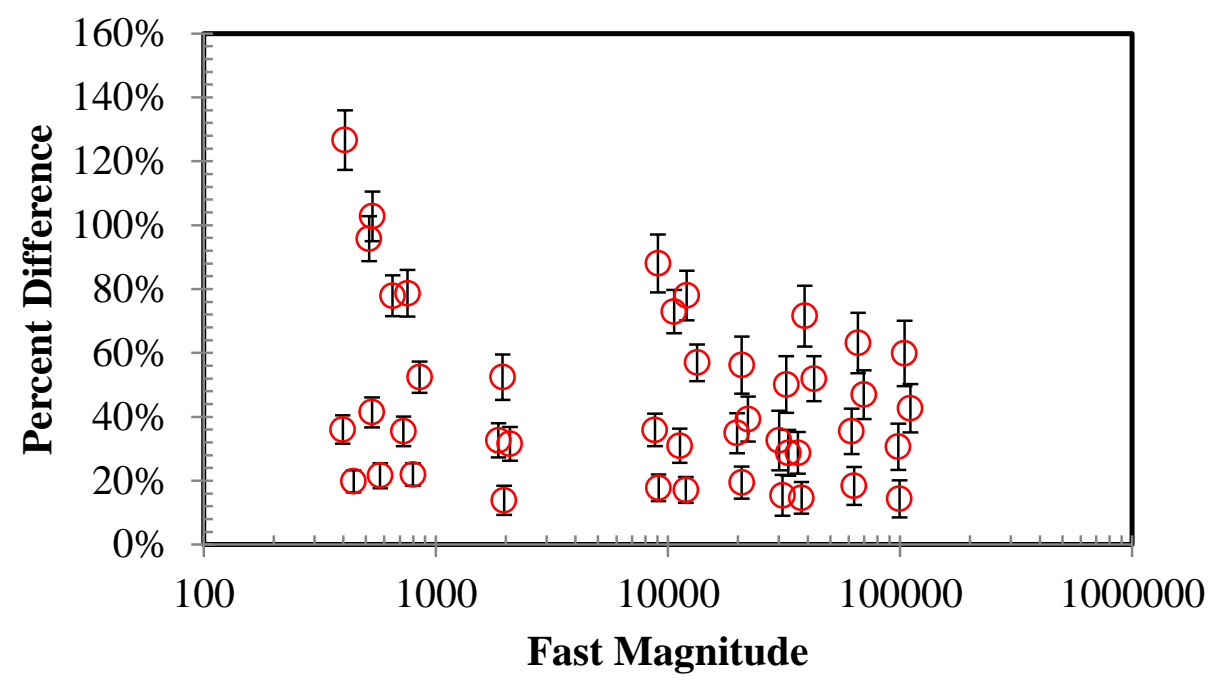

Figure 5-19. Percent difference between fast/slow magnitude for complete and diverted

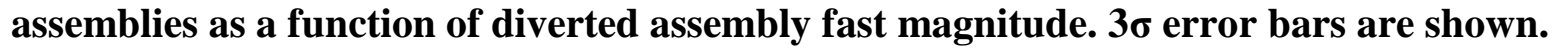

Multiplication alone is a useful indicator when changing from fuel to stainless steel because it is still predicted quite accurately regardless of whether or not there is a diversion. The plot of early dieaway time vs. multiplication was only slightly more scattered than that of the complete cases, with a standard deviation that was approximately $1 \%$ worse. The multiplication change with NU replacement is so small, however, that multiplication cannot be used to identify NU replacements. Within single assembly types large changes in net multiplication from the complete assemblies were observed depending on the replacement material and diversion scheme. This change was reflected in the early die-away time, however, allowing for continued accurate prediction of multiplication. The plot of multiplication vs. early die-away is shown in Figure 5-20 for all diversion scenarios. $1 \sigma$ error bars are shown on the early die-away times. The equation of the quadratic fit is very close to the fit from complete assemblies which is why those calibration constants could be used and still obtain good approximations of multiplication. 


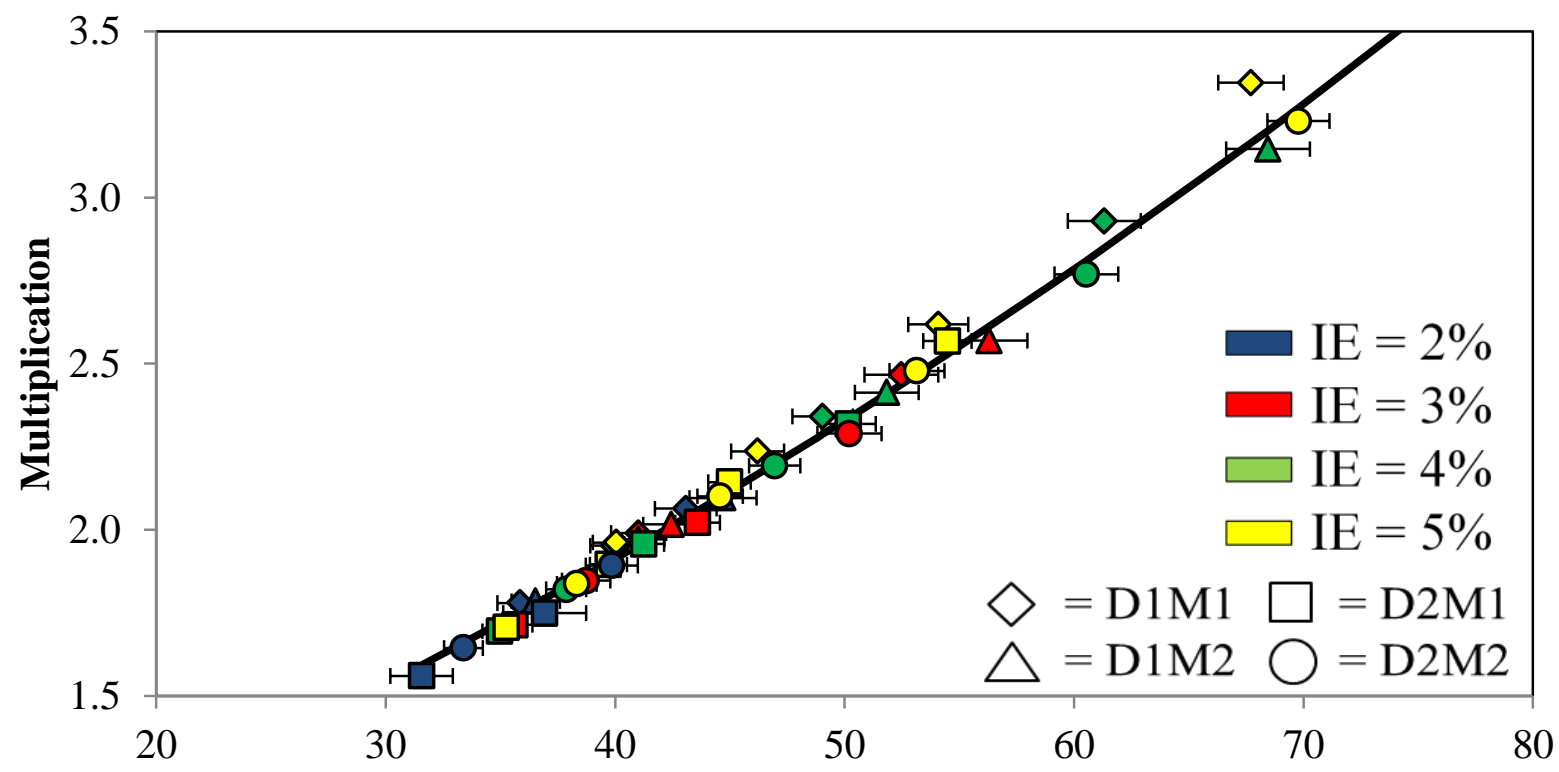

Early Die-Away Time [ $\mu \mathrm{s}]$

Figure 5-20. Multiplication vs. early die-away time for assemblies with diversion schemes. Solid curve indicates quadratic fit obtained from complete SFL2a assemblies.

Though multiplication was higher for complete assemblies (as expected) the values still fell on the same curve as the diverted cases, which is what would be required to identify assemblies with diversions. If the diverted assemblies instead required a different calibration curve then the method could not be applied. The multiplication decreased for assemblies with stainless steel pins replacing diverted center pins, and it stayed mostly the same for assemblies with natural uranium replacing diverted dispersed pins. This makes sense-more pins removed from a single location will have a more noticeable change in multiplication because neutrons entering that region will have a difficult time continuing through fission chains. Therefore assuming there was a declared multiplication value from the operator, or that a declared value could be obtained from simulation of the assembly with declared parameters, a diversion and replacement with SS could be identified by a large drop in multiplication in experiment relative to the declared value. However the fast/slow magnitude appears to be the best indicator in the reference assembly approach.

\subsubsection{Changes in Total Plutonium Mass Approach}

It has been shown, by means of MCNP simulations, that total Pu content can be determined by DDA- and DDSI-based instruments [33,34] because both methods can measure assembly multiplication and passive neutron emission rates (see Section 8.3). In this work, the DDSI 
instrument was used to leverage this capability and cross check the total Pu content based on simulated measurements and an operator's implicit or explicit declaration to identify SFAs from which fuel pins have been illicitly removed.

Due to the sensitive nature of this work, results are not presented here but are given in [79]. MCNPbased simulations were performed of assays of 44 PWR SFAs of the Westinghouse type (17x 17 pins) that have various irradiation parameters. Forty fuel pins were removed from these SFAs and substituted with pins of natural uranium (NU) or stainless steel (SS). It is important to note that this method of pin diversion detection does not aim to calculate absolute Pu content in assemblies, but rather relative $\mathrm{Pu}$ content in a set of assemblies with varied operating parameters. It can be safely assumed that the effectiveness of the method depends on the uncertainty of the actual measurements, the uncertainty inherent to the method itself, and the accuracy of the operator's declaration. The first can be mitigated by appropriate engineering and adequate statistics and should not account for more than a couple percent. The error inherent to the method is approximately 2-5\%. The latter issue of operator declaration accuracy is generally more uncertain. Currently, it is widely accepted [81] that based on the known irradiation history of a given SFA, the total Pu content can be estimated through various burnup codes with an accuracy of 5\%-10\%. Such a large uncertainty may seem to be rather limiting; however, it should be noted that the NDA instrument used for Pu determination will likely use, for its own calibration, a calculated Pu content from a subset of the SFAs to be assayed. Therefore, it can be reasonably expected that the Pu content determined from the measurement will be burdened by the same or a very similar systematic error as the value of Pu content used for the calibration and the value of $\mathrm{Pu}$ from the operator's declaration, assuming the same burnup codes are used. Considering such a scenario, $72 \%$ of assemblies with SS replacement pins investigated could be identified as "abnormal" with the difference between the measurement-based determination of total Pu content and the one based on operator declaration exceeding $3 \sigma$ confidence levels. Because of the lack of change in multiplication from complete assemblies to those with NU replacements, the relative Pu content method cannot be used to confidently detect diversions where NU is the replacement material. Detailed explanation of this work including results and analysis can be found in [79]. 


\subsection{Initial Enrichment and Burnup Determination}

Determining the basic fuel assembly parameters including initial enrichment (IE) and burnup (BU) remains one of the primary goals when measuring spent nuclear fuel [13]. Knowledge of BU is essential for criticality safety and burnup credit determination and is thus of interest both to the IAEA and national regulating bodies as well as private sector plants in the US and elsewhere [82]. NDA technologies such as the Fork Detector and Cherenkov Viewing Device, as well as the experimental NGSI-SF techniques, have been developed with the goal of verifying operator declarations including IE and BU [83].

One of the goals of the NGSI-SF project has been to integrate different NDA techniques to accomplish these measurements, because single neutron or gamma measurement method typically does not collect enough information to independently determine IE and BU without a-priori knowledge [84]. As such, the DDSI instrument with neutron coincidence counting alone will likely not be able to characterize an assembly of unknown origin with respect to IE and BU. However, there are several interesting physical relationships between these parameters that could be exploited by the DDSI method in combination with another NDA technology, and they are explored here with the intent of identifying potential measured signals that could be used in combination with trusted declarations to measure the basic fuel parameters.

\subsubsection{Total Neutron and Gamma Rates}

DDSI detects neutrons with $56{ }^{3} \mathrm{He}$ detectors and can also measure the total gamma rate with the use of two ion chambers. The neutron singles rate is known to trend with BU raised to a power, expressed in equation (14)

$$
S \propto B U^{\beta}
$$

Where $S=$ Singles and $\beta=$ a calibration constant depending on the assembly's geometry and irradiation history [14]. The total gamma rate trends with cooling time and burnup with the expression in equation (15)

$$
\frac{T G}{B U}=a(C T)^{b}
$$


Where $T G=$ total gamma, and $a$ and $b$ are fitting constants [14]. This relation is demonstrated for cooling times in Figure 5-21. The total neutron rate vs the total gamma rate may also provide an indication of BU that is IE and CT dependent [85].

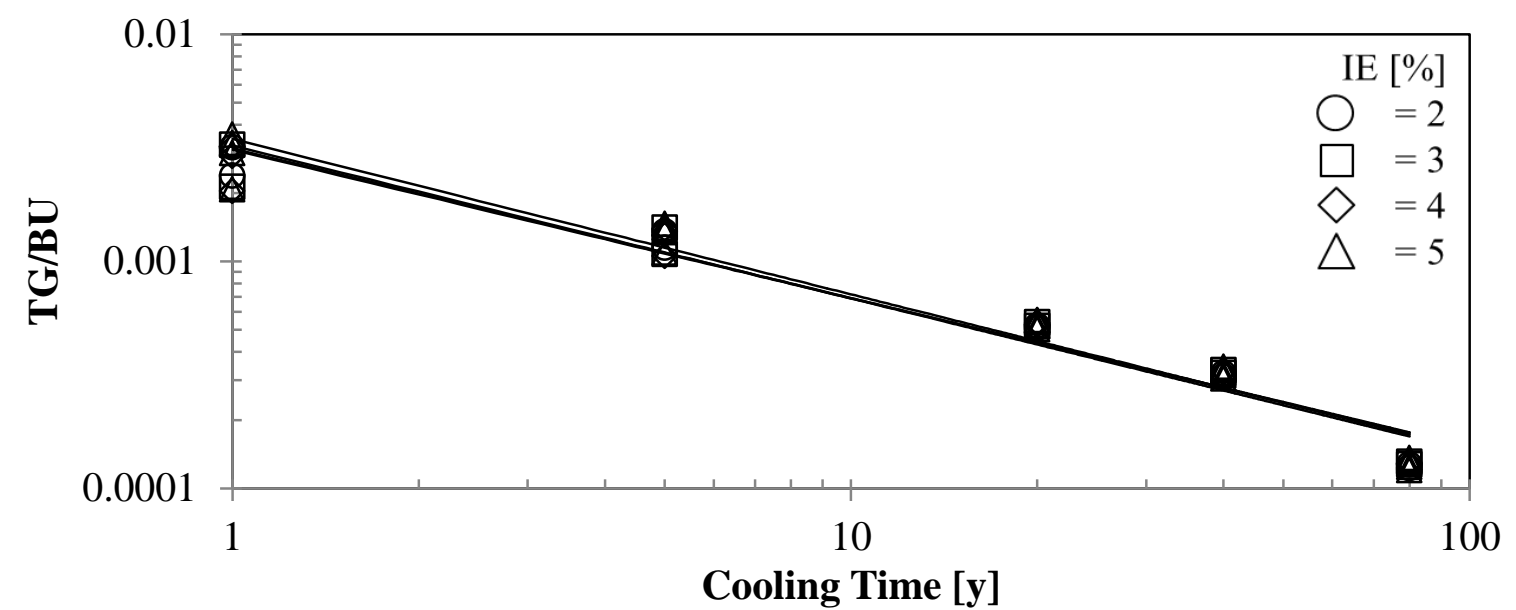

Figure 5-21. Relationship between total gamma, burnup, and cooling time. Power fits for each enrichment overlap, indicating that a single fit is appropriate.

It was found that $\mathrm{BU}^{4} / \mathrm{IE}^{2}$ also trended with the singles rate as measured with DDSI as a cooling time-dependent second order polynomial as shown in Figure 5-22. Thus if cooling time were known and the operator declared value for IE could be trusted or verified through other means, BU could be determined with an RMS error of $12 \%$. This method differs from the previous figure because the total neutron signal is used instead of total gamma.

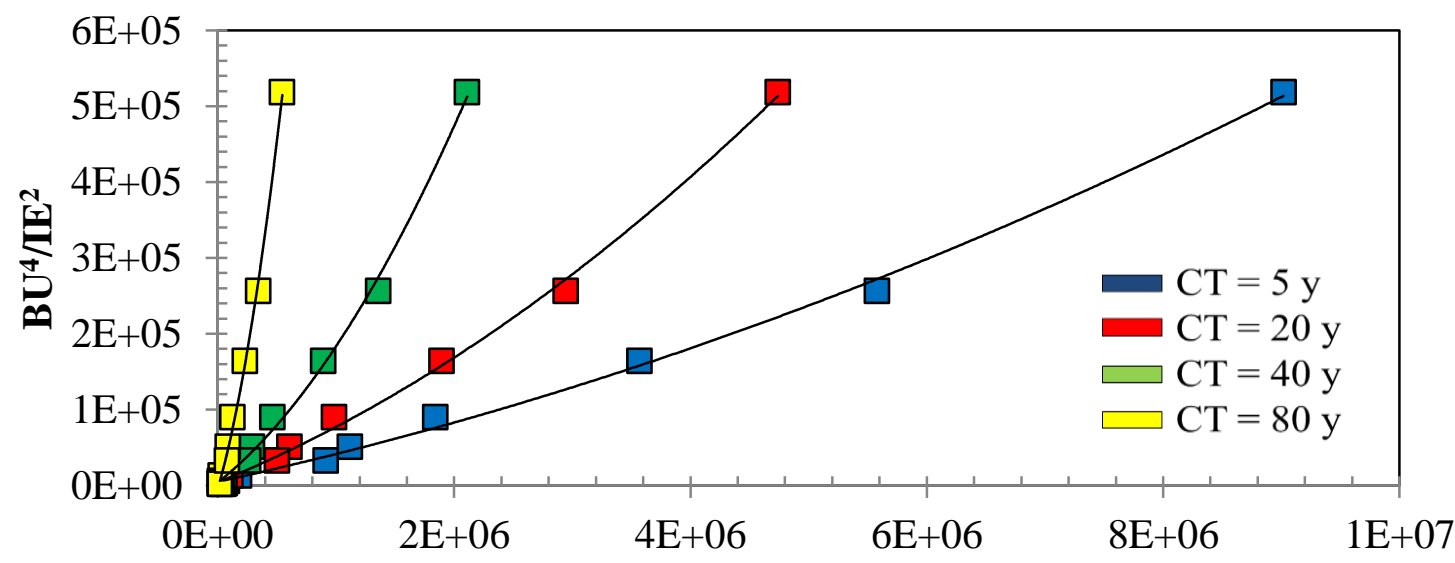

Singles Rate [cps]

Figure 5-22. Relationship between burnup, initial enrichment, and neutron singles rate for each cooling time for spent nuclear fuel assemblies in SFL2a. 
While the total gamma relation is simpler because it does not depend on initial enrichment, the total gamma signal comes only from the exterior rows of pins in the fuel assembly and thus could face issues such as center pin diversions and large burnup gradients. In addition, the total gamma vs CT relationship becomes essentially flat after approximately 20 years of cooling.

It has been shown that there is a correlation between multiplication and early die-away time of the simulated Rossi-alpha distribution from DDSI [9]. It has also been shown that cooling time can be well approximated by total gamma measurements [1]. Assuming that these two factors have been measured, burnup and cooling time values can be predicted using two additional measured parameters: total neutron coincidences from 1-200 $\mu$ s following a trigger event (called total doubles), and total neutron coincidences from 1-41 $\mu$ s following a trigger event (called fast doubles).

The amount of fissile content in an assembly is a result largely of the degree it is burned and the initial uranium enrichment. However, these two factors cannot be determined independently because a single initial enrichment burned to different degrees will not yield the same multiplication or fissile material mass. Therefore BU and IE must be treated as dependent variables in analysis of related, measurable quantities. The minimum number of equations necessary to predict these variables becomes two instead of one when they are treated as dependent.

As a result, relationships must be identified between BU, IE, and two different measurable parameters. Results of the simulations of assay of SFL2a assemblies are used for analysis. The first relationship identified is that $I E^{1.4} / B U$ is approximately linearly proportional to multiplication as shown in Figure 5-23. 


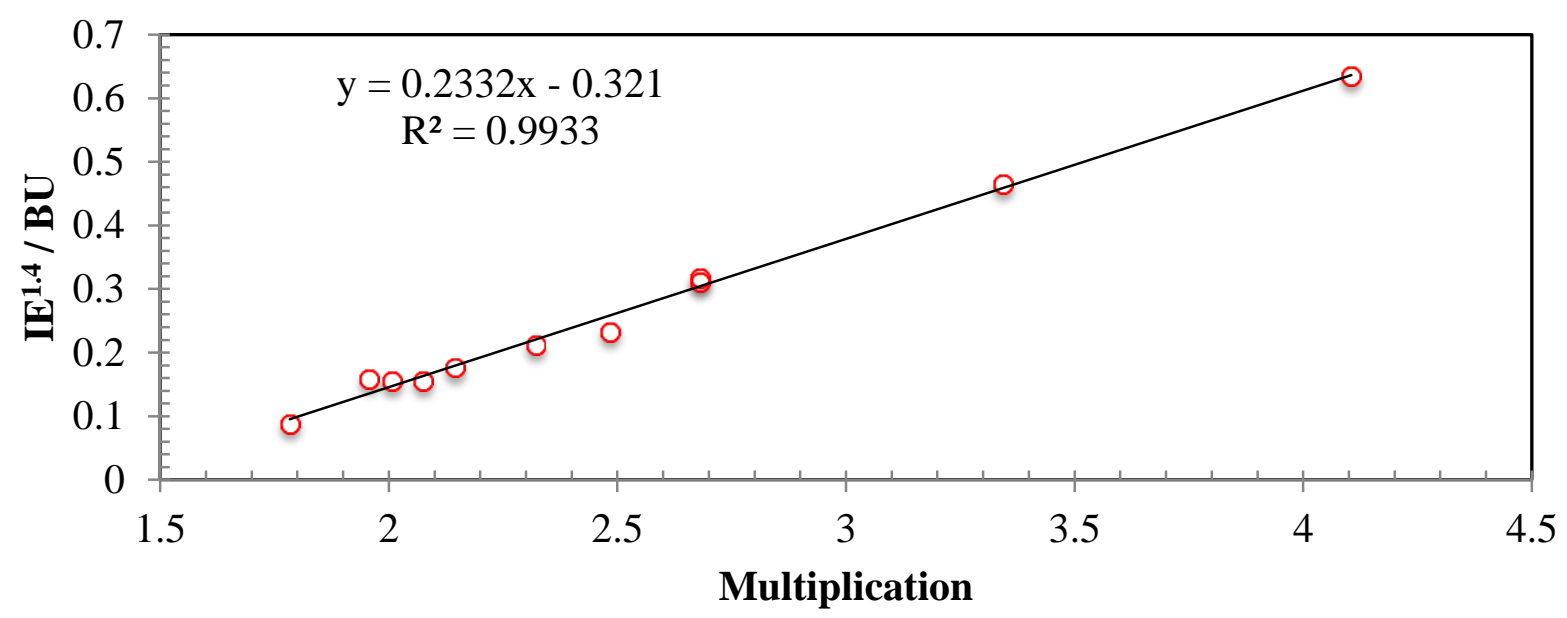

Figure 5-23. Linear relationship between $\mathrm{IE}^{1.4} / \mathrm{BU}$ and multiplication.

The second measurable identified to correlate with $\mathrm{BU}$ and IE is the ratio of fast doubles to total doubles, where fast doubles is the integral of the early time domain of the RAD $(4-60 \mu \mathrm{s})$ and total doubles is the integral of the entire RAD. Here a second order polynomial correlation is observed between BU/IE and fast/total, as shown in Figure 5-24.

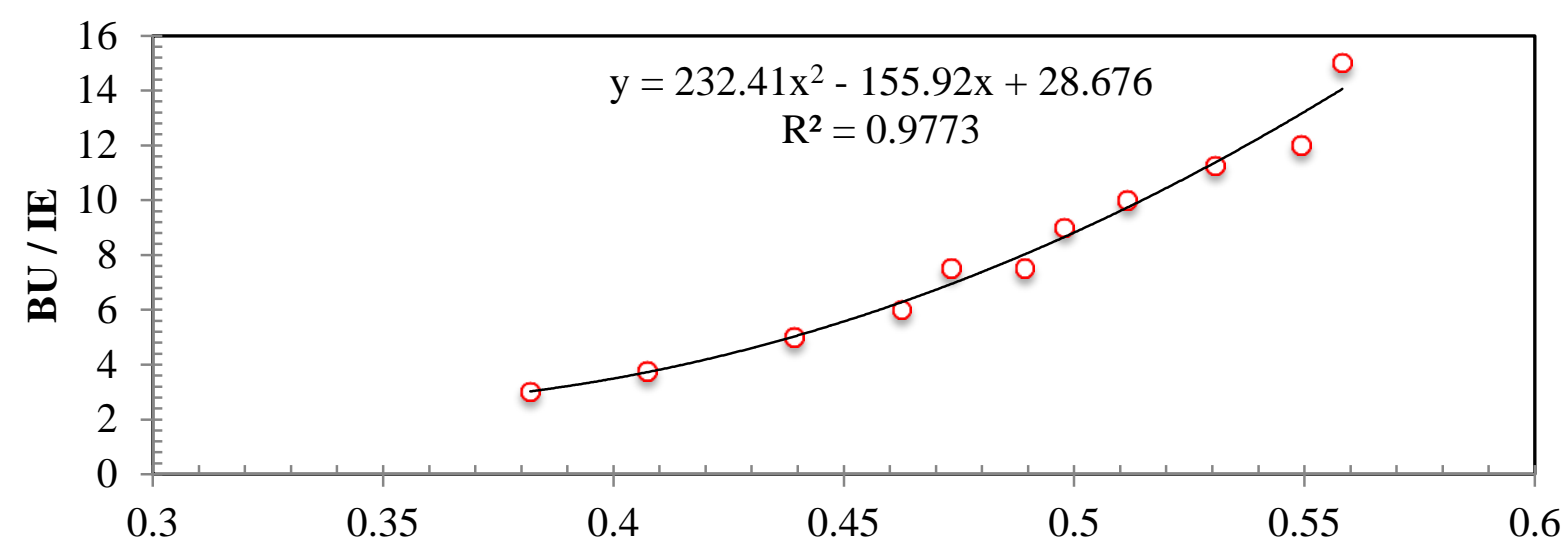

Fast Doubles / Total Doubles

Figure 5-24. 2nd order polynomial relationship between BU/IE and Fast/Total doubles.

Now that there are two equations and two unknowns, burnup and initial enrichment can be solved for. The results of these predictions are given in Table 5-VII. 
Table 5-VII. Predicted values of BU and IE with constant/known cooling time of 5y

\begin{tabular}{ll|ll|ll}
\hline $\begin{array}{l}\text { IE } \\
{[\%]}\end{array}$ & $\begin{array}{l}\text { BU } \\
{[\mathbf{G W d} / \mathbf{t U}]}\end{array}$ & $\begin{array}{l}\text { Predicted } \\
\text { IE [\%] }\end{array}$ & $\begin{array}{l}\text { Predicted } \\
\text { BU } \\
{[\mathbf{G W d} / \mathbf{t U}]}\end{array}$ & $\begin{array}{l}\text { Relative } \\
\text { Diff: } \\
{[\%]}\end{array}$ & $\begin{array}{l}\text { Relative } \\
\text { [iff: BU } \\
{[\%]}\end{array}$ \\
\hline $\mathbf{2}$ & $\mathbf{1 5}$ & 2.06 & 15.51 & $3 \%$ & $3 \%$ \\
$\mathbf{3}$ & $\mathbf{1 5}$ & 2.98 & 15.12 & $-1 \%$ & $1 \%$ \\
$\mathbf{4}$ & $\mathbf{1 5}$ & 4.03 & 15.16 & $1 \%$ & $1 \%$ \\
$\mathbf{5}$ & $\mathbf{1 5}$ & 4.97 & 15.25 & $-1 \%$ & $2 \%$ \\
\hline $\mathbf{2}$ & $\mathbf{3 0}$ & 1.55 & 21.16 & $-23 \%$ & $-29 \%$ \\
$\mathbf{3}$ & $\mathbf{3 0}$ & 2.80 & 26.98 & $-7 \%$ & $-10 \%$ \\
$\mathbf{4}$ & $\mathbf{3 0}$ & 4.51 & 32.06 & $13 \%$ & $7 \%$ \\
$\mathbf{5}$ & $\mathbf{3 0}$ & 6.05 & 36.47 & $21 \%$ & $22 \%$ \\
\hline $\mathbf{4}$ & $\mathbf{4 5}$ & 3.42 & 40.15 & $-15 \%$ & $-11 \%$ \\
$\mathbf{5}$ & $\mathbf{4 5}$ & 5.61 & 51.21 & $12 \%$ & $14 \%$ \\
\hline $\mathbf{5}$ & $\mathbf{6 0}$ & 4.53 & 59.86 & $-9 \%$ & $0 \%$ \\
\hline Average (of absolute values of differences): & $\mathbf{9 \%}$ & $\mathbf{9 \%}$ \\
\hline
\end{tabular}

\subsection{Conclusions}

High-fidelity simulations of the DDSI instrument were performed to develop and test various spent fuel analysis algorithms. Simulations and subsequent analysis of calculated RADs has demonstrated the potential to determine multiplication of a spent fuel assembly with high accuracy. The die-away time of the RAD in the early time domain (4-52 $\mu \mathrm{s})$, that can be fitted with a single exponential function, exhibits a nearly linear correlation to the multiplication of the assayed spent fuel assembly. This die-away time is a function of the interplay between the fast and the slow components of the RAD that are produced by detected coincident fission neutrons and detected coincident fission chain neutrons, respectively. In simulations of the base library, SFL2a, the multiplication is determined from the early die-away time with a variance of $0.7 \%$ across the 44 assemblies. This is an improvement upon past methods of multiplication determination with the DDSI method applied to spent nuclear fuel which, unlike the present method, required knowledge of the $(\alpha, n)$ source term to reduce scatter from changing assembly parameters. 
Results further showed that the recently developed approach of total plutonium determination based on measurement of SFA multiplication and passive neutron count rate is also applicable for use with the DDSI instrument. In the context of nondestructive assay of spent nuclear fuel by the DDSI instrument, the multiplication is measured in terms of the early die-away time of the Rossi-alpha distribution, and the passive neutron count rate is measured in the form of the detected singles rate. Overall, considering the CT known or constrained and discounting any systematic errors of real-life measurements, simulations show that the total plutonium content of a spent fuel assembly from the base library SFL2a is determined with the RMS error of $2.1 \%$ without explicit knowledge of the assembly initial enrichment or burnup. This error will likely be larger when uncertainty in Pu content in the models and experimental uncertainty are introduced.

The ability to reliably identify assemblies that have had less than $50 \%$ of their pins illicitly removed or replaced has been considered a high priority in the international safeguards community. Two methods were proposed to accomplish this-1) compare the signal of a measured assembly to a reference assembly and 2) compare the total $\mathrm{Pu}$ content in spent fuel determined from assembly assay with the total $\mathrm{Pu}$ content stated in or based on the operator's declaration. In the reference assembly approach, the fast/slow magnitude parameter was able to identify, with better than $3 \sigma$ confidence, $100 \%$ of assemblies from which 40 pins (i.e., 14\%-16\% of total Pu content) have been removed and pins containing only stainless steel or natural uranium were used as substitutes. It was also demonstrated - by means of MCNP-based simulations - that the DDSI instrument can use the self-consistent approach to identify, with $3 \sigma$ confidence, $73 \%$ of fuel assemblies with diversions investigated within this thesis. This is done using a relative plutonium mass determination as described in [79].

Burnup, initial enrichment, and cooling time remain the most difficult, if not impossible, parameters to determine with neutron coincidence counting alone. Multiple methods were proposed and would hypothetically be able to determine these parameters, however the already large uncertainties in simulation space would likely be increased in experiments and could make determination impossible. When photon energy measurements are possible in conjunction with DDSI measurements, new possibilities for different analysis techniques arise, and these may be investigated in future work. 
In all of the preceding methods based on high fidelity simulations, additional uncertainty will likely be introduced in real world measurements. The close correlations observed, however, lend merit to the proposed methods and make them attractive options for further experimental validation. 


\section{Chapter 6. INSTRUMENT CHARACTERIZATION EXPERIMENTS}

The DDSI instrument was built in the end of 2014 and was then extensively tested with different sources and fresh fuel configurations at LANL. The instrument was characterized and benchmarked through a series of simple source measurements in air and water. A high count rate experiment was also performed to test the limits of the electronics and data collection capabilities. The characterization experiments serve three primary purposes: 1) Characterize the instrument and determine basic operating parameters such as deadtime, die-away time and efficiency, 2) benchmark the simulations of DDSI, and 3) evaluate the readiness of the instrument for deployment and assay of spent nuclear fuel.

The DDSI method requires a neutron source accompanied by fissile material to initiate fission chains and observe the die-away of the neutron population in time. When assaying induced spent fuel, the neutron source is self-contained in the abundant spontaneous fission isotopes such as ${ }^{244} \mathrm{Cm}$ and ${ }^{240} \mathrm{Pu}$. The characteristic die-away time observed from different assemblies with varying initial enrichment, burnup, and cooling time allows us to determine SFA properties such as multiplication and total Pu content $[9,34]$. When assaying fresh fuel, a spontaneous fission driver source must be added to provide the interrogating neutrons since ${ }^{235} \mathrm{U}$ and ${ }^{238} \mathrm{U}$ spontaneous fission rates are too low for any practical use with the DDSI method.

The DDSI instrument contains $56{ }^{3} \mathrm{He}$ detectors in four stainless steel enclosures surrounding a central cavity. Figure 6-1 shows one of the open detector enclosures. Each tube is connected to an individual preamplifier with its own signal lead that is fed through conduit out to the data acquisition system. Four additional detector slots, one in each detector pod, are used for alternate detectors including two ion chambers and two ${ }^{3} \mathrm{He}$ tubes with experimental preamplifiers. Additional $\mathrm{Cd}$ lining is added to the alternate detector slots in order to maintain the same flux in the neighboring tubes as if a standard ${ }^{3} \mathrm{He}$ counter was in the slot. 


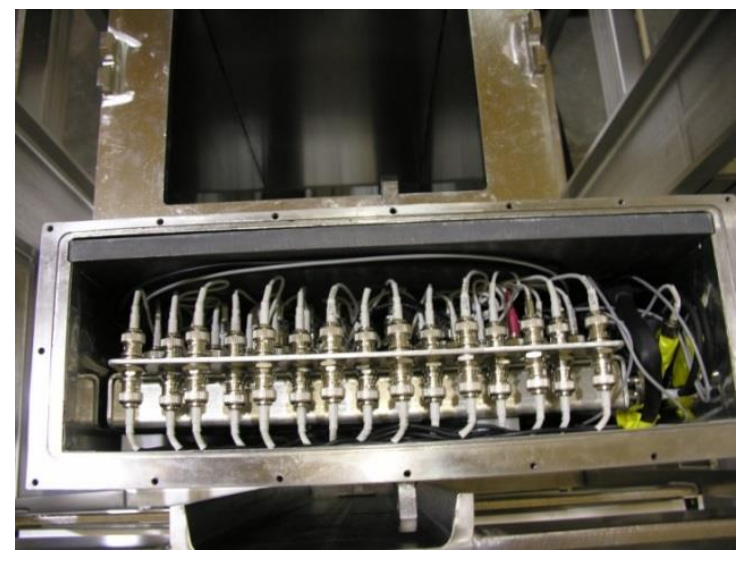

Figure 6-1. Picture of DDSI instrument with detector pods open. Detector pods are sealed with lead and stainless steel covers before measurements take place.

For measurements, lids were added to each detector pod enclosure and sealed so that the instrument could be placed in water. The 25 meters of cabling for each detector pod is routed through a watertight conduit, the fitting of which was tightened to torque specifications and the detector pods were pressure tested to 30 psi prior to instrument assembly. The long conduits enable the instrument to be placed in the bottom of a spent fuel pool. The sealed instrument in the measurement tank is shown in Figure 6-2. Instrument specifications including detector pitch, enclosure thickness, and others can be found in 0 .

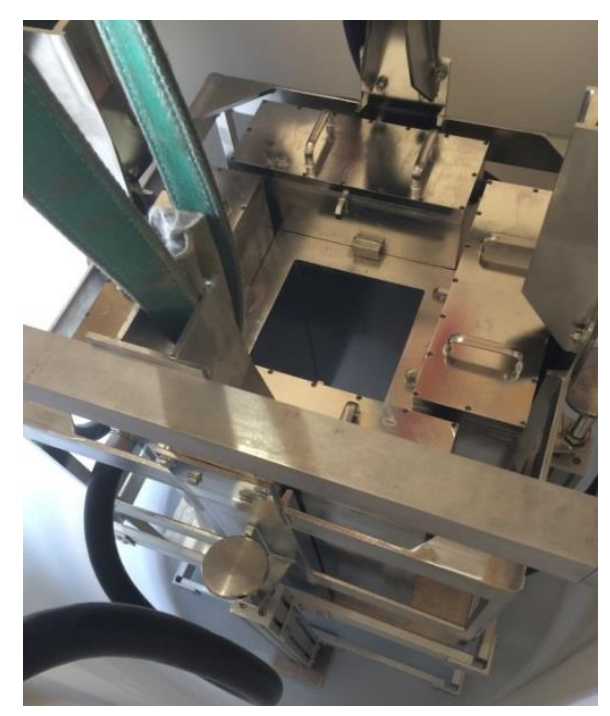

Figure 6-2. Sealed DDSI instrument in measurement tank. 


\subsection{Experimental Setup}

In July of 2014, all 56 sets of ${ }^{3} \mathrm{He}$ detectors and corresponding preamplifiers in use and the six spare sets were gain matched. The preamplifiers were all numbered with corresponding tubes, and they were all set to match Set 1 . For the match to be considered adequate, the ratio of counts at $1650 \mathrm{~V}$ over counts at $1500 \mathrm{~V}$ had to be within $1.5 \%$ of the ratio from Set 1 . High voltage plateaus were measured for sets 1, 10, 20, 30, and 40. These plateaus are shown in Figure 6-3.

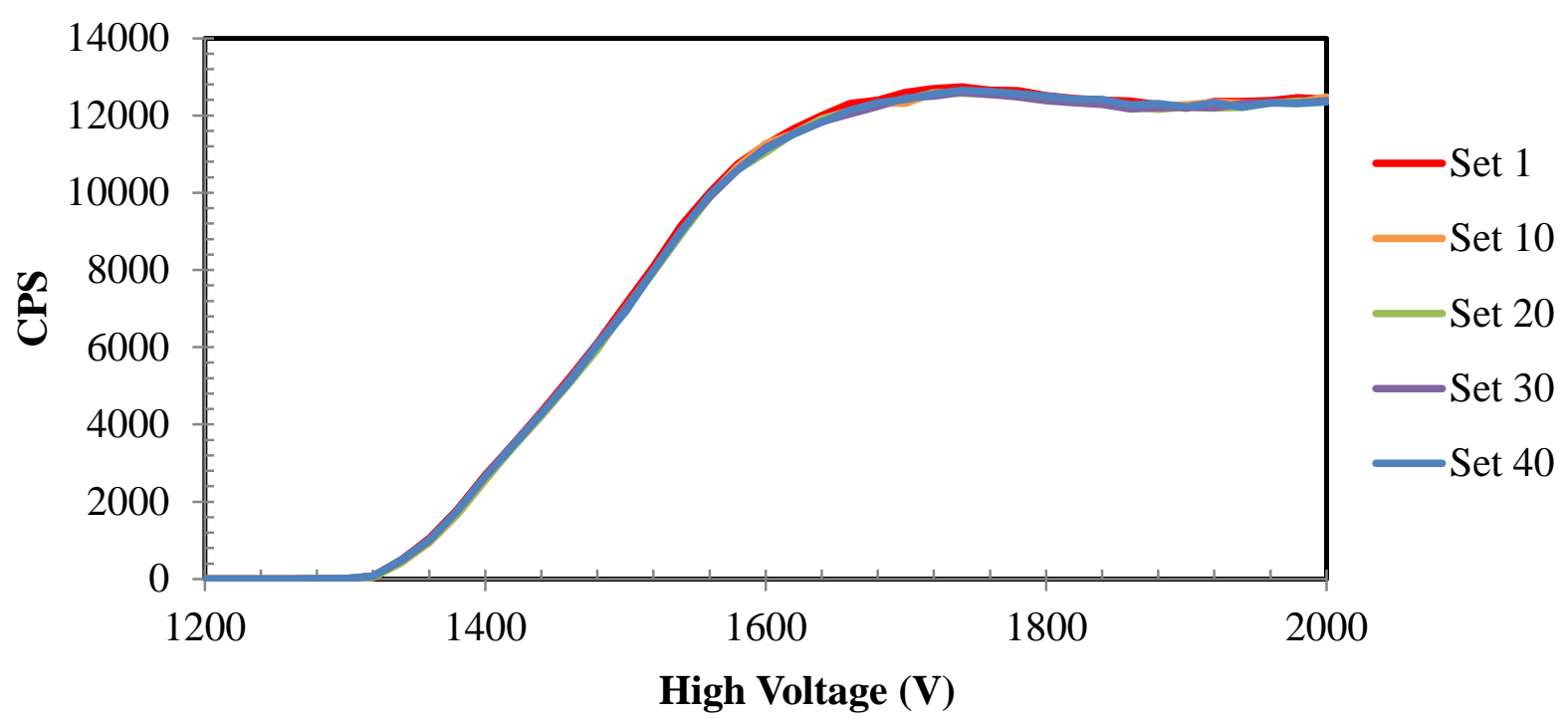

Figure 6-3. High Voltage plateaus for detector/preamp sets 1, 10, 20, 30, and 40. Agreement is within $1.5 \%$ between the different sets.

A series of ${ }^{226} \mathrm{Ra}$ measurements were performed to determine the optimal operating high voltage assuming high gamma flux (as will be the case with spent fuel) and $1620 \mathrm{~V}$ was selected. The ${ }^{3} \mathrm{He}$ tubes and preamplifiers were also tested for temperature stability under varying heat conditions and $1620 \mathrm{~V}$ was confirmed to be a high voltage at which the possible temperature drifts were sufficiently low. These measurements are described in Chapter 2.

The DDSI instrument was constructed with the ${ }^{3} \mathrm{He}$ tubes and preamplifiers and ion chambers in the polyethylene banks. Detector cables were connected to the attached BNC connector ports inside the detector pod enclosures and the $25 \mathrm{~m}$ connector cables were fed out of each bank through conduit to the DAQ. The conduit also houses the HV and LV cables to power the detectors in each bank, and a spare HV, LV, and signal cable was added to each cable bundle. The instrument itself was lifted by 
crane and lowered into a 500 gallon tank where it was supported by four stainless steel legs before any water was added. For initial measurements, the single $51.9 \mu \mathrm{Ci}^{252} \mathrm{Cf}$ source was placed in the center of the instrument. It is important that the source be centered in the instrument for benchmarking purposes so it was secured in place for measurements in air. The data were collected using list mode data acquisition system with a single input from each detector in the instrument and a signal splitter was also used to feed the data into a shift register module for analysis with INCC software on a separate laptop. List-mode data were analyzed with FastTapX software which is capable of producing RADs as described in detail in Section 3.4, utilizing user defined time window widths, and calculating doubles and singles rates based on the user-defined PD and GW. Window width is the length of the RAD histogram after trigger, and time bins are the bin widths used in the histogram. The singles and doubles rates from the list mode data were also compared with the INCC shift-register results.

\subsection{Comparison of Shift Register and List Mode Results}

The Canberra JSR-15 shift register has been verified and validated many times in the past and could thus be used as a reference. The results from list mode data collection and analysis were compared to those from the shift register to confirm that the list mode data were being collected correctly. Measurements were taken with various sources including strong and weak ${ }^{252} \mathrm{Cf}$ sources and an AmLi source in the center of the instrument in air. A predelay of $1.5 \mu$ s was used along with a gate width of $24 \mu$ s which was chosen to be commensurate with the instrument die-away time, as has been shown to be optimal [86]. The simulated data must use a predelay of $1.6 \mu$ s instead of $1.5 \mu \mathrm{s}$ because RAD bin widths must be a multiple of the sampling frequency, which in this case is $5 \mathrm{MHz}$. Therefore the RAD bin widths must be a multiple of $0.2 \mu \mathrm{s}$. Results are given in Table 6-I and are published in [87]. 
Table 6-I Comparison of the JSR-15 results with list mode and RAD analyzed or created by FastTapX.

\begin{tabular}{|c|c|c|c|}
\hline & & Singles (cps) & Doubles (cps) \\
\hline \multirow{3}{*}{$\begin{array}{l}\text { AmLi and }{ }^{252} \text { Cf together, } \\
1.5 \mu \mathrm{s} \text { PD, } 24 \mu \mathrm{s} \text { GW }\end{array}$} & JSR-15 (1.5 $\mu \mathrm{s}$ PD, $24 \mu \mathrm{s}$ GW) & $40,304 \pm 10$ & $3,398 \pm 14$ \\
\hline & List Mode (1.5 $\mu \mathrm{s}$ PD, $24 \mu \mathrm{s}$ GW) & $40,337 \pm 14$ & $3,458 \pm 35$ \\
\hline & RAD (1.6 $\mu \mathrm{s}$ PD, $24 \mu \mathrm{s}$ GW) & $40,337 \pm 12$ & $3,440 \pm 16$ \\
\hline \multirow{3}{*}{$\begin{array}{l}\text { Two strong }{ }^{252} \mathrm{Cf} \\
\text { sources, center, } 1.5 \mu \mathrm{s} \\
\text { PD, } 24 \mu \mathrm{s} \text { GW }\end{array}$} & JSR-15 (1.5 $\mu \mathrm{s}$ PD, $24 \mu \mathrm{s}$ GW) & $685,585 \pm 30$ & $74,960 \pm 167$ \\
\hline & List Mode $(1.5 \mu \mathrm{s}$ PD, $24 \mu \mathrm{s}$ GW) & $686,003 \pm 51$ & $74,973 \pm 222$ \\
\hline & RAD (1.6 $\mu \mathrm{s}$ PD, $24 \mu \mathrm{s}$ GW) & $686,004 \pm 36$ & $74,887 \pm 183$ \\
\hline \multirow{3}{*}{$\begin{array}{l}\text { One }{ }^{252} \text { Cf source (3097), } \\
\text { center, } 1.5 \mu \mathrm{s} \text { PD, } 24 \mu \mathrm{s} \\
\text { GW }\end{array}$} & JSR-15 (1.5 $\mu \mathrm{s}$ PD, $24 \mu \mathrm{s}$ GW) & $348,359 \pm 22$ & $39,213 \pm 86$ \\
\hline & List Mode $(1.5 \mu \mathrm{s}$ PD, $24 \mu \mathrm{s}$ GW) & $348,357 \pm 47$ & $39,201 \pm 209$ \\
\hline & RAD (1.6 $\mu \mathrm{s}$ PD, $24 \mu \mathrm{s}$ GW) & $348,353 \pm 26$ & $39,146 \pm 94$ \\
\hline \multirow{3}{*}{$\begin{array}{l}\text { One }{ }^{252} \text { Cf source }(3098) \text {, } \\
\text { center, } 1.5 \mu \mathrm{SD}, 24 \mu \mathrm{s} \\
\text { GW }\end{array}$} & JSR-15 (1.5 $\mu \mathrm{s}$ PD, $24 \mu \mathrm{s}$ GW) & $341,320 \pm 21$ & $38,190 \pm 22$ \\
\hline & List Mode (1.5 $\mu \mathrm{s}$ PD, $24 \mu \mathrm{s}$ GW) & $341,296 \pm 45$ & $38,290 \pm 187$ \\
\hline & RAD (1.6 $\mu \mathrm{s}$ PD, $24 \mu \mathrm{s}$ GW) & $341,322 \pm 25$ & $38,226 \pm 91$ \\
\hline
\end{tabular}

Agreement was within statistical deviation and thus list mode data collection was determined to be accurate for future measurements and implementation of fuel analysis algorithms.

\subsection{Deadtime Determination}

Deadtime was determined with the two source method. In this method, two strong sources are measured together initially in the center of the instrument in air. One source is then removed and replaced with a dummy source, and the measurement is repeated. The same is then done for the other source. The decrease in count rate when two sources are measured together as opposed to two sources measured separately with the results added together can be used to determine deadtime. The Two ${ }^{252} \mathrm{Cf}$ sources used each had an activity of $677.1 \mu \mathrm{Ci}$. To correct for deadtime exactly, the following equation can be used

$$
S_{o}=S_{m} e^{d S_{o}}
$$


where $S_{o}=$ the corrected count rate, $S_{m}=$ measured count rate, and $d=$ singles deadtime [1]. This expression requires a priori knowledge of the corrected count rate, which cannot be explicitly known. Thus an approximation is used where the exponential factor $d S_{0}$ is replaced with $\delta_{t} S_{m}$, where:

$$
\delta_{t}=\frac{A+B S_{m}}{4}
$$

where $A$ and $B$ are determined empirically to minimize the difference between the expected and actual deadtime corrected count rates. The factor of 4 is an empirical correction factor that is widely used in the field, and there is no mathematical derivation for its use. The resultant equation for deadtime correction on the Singles rate is:

$$
S_{o}=S_{m} e^{\frac{\left(A+B S_{m}\right) S_{m}}{4}}
$$

The Doubles deadtime correction is similar, and is given by:

$$
D_{o}=D_{m} e^{\left(A+B S_{m}\right) S_{m}}
$$

Using these expressions, the singles deadtime was determined to be approximately 23 ns for 56 detector channels. The doubles deadtime was equal to $92 \mathrm{~ns}$, which is considerably lower than that of typical ${ }^{3} \mathrm{He}$-based neutron coincidence counters, which is on the order of $200 \mathrm{~ns}$ [88].

\subsection{Die-Away Time and Efficiency Determination}

A single ${ }^{252} \mathrm{Cf}$ source in air was used to characterize the instrument's die-away time and efficiency. A Rossi-alpha distribution was produced with FastTapX software and analyzed with Python ${ }^{1}$ [89]. The source used was A7-868 which had a yield of $211,380 \mathrm{cps}$ on $4 / 14 / 2015$, the date of the measurement. An experimental RAD produced with FastTapX and analyzed with Python is shown in Figure 6-4. $1 \sigma$ error bars are shown on the doubles rates.

\footnotetext{
${ }^{1}$ Python analysis scripts can be found in Appendix C.
} 


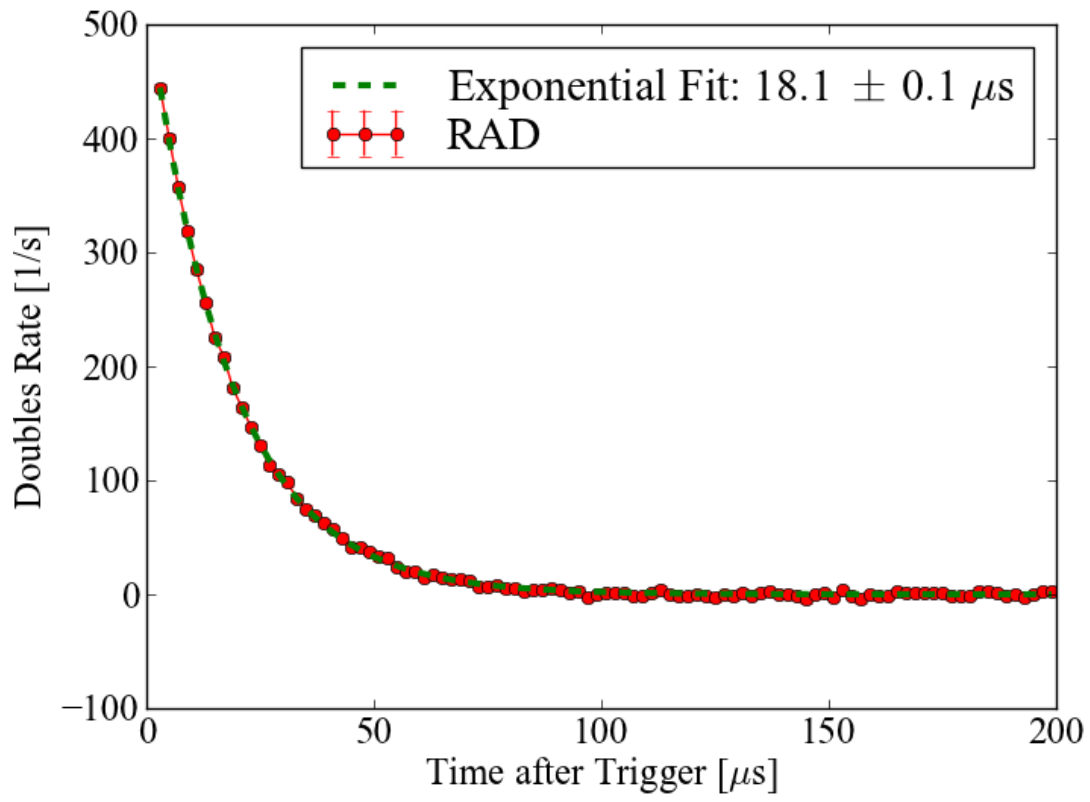

Figure 6-4. RAD from experimental list mode data with $2 \mu$ s bins. Die-away time from fit to RAD is $18.1 \mu s$.

The die-away time as determined from a single exponential fit using non-linear least squares to the RAD with $2 \mu$ s bins was $18.1 \pm 0.1 \mu \mathrm{s}$. The bin width of $2 \mu$ s was used for comparison with simulation and for simple exclusion of the predelay period. The predelay allows for exclusion of deadtime and recovery time effects. Detection efficiency was also determined from this measurement with deadtime corrected singles rate. The correction that was used for the singles count rate based on the deadtime results from Section 6.3:

$$
S_{o}=S_{m} e^{\frac{\left(0.92 \times 10^{7}+0.21 \times 10^{-14} \times S_{m}\right) S_{m}}{4}}
$$

Using the singles rate with deadtime correction implemented, the singles rate was $25,335 \pm 160 \mathrm{cps}$ and the detector efficiency for a point source in the center of the instrument in air was $12.0 \% \pm$ $0.4 \%$, assuming a $3 \%$ uncertainty in the accuracy of the source strength.

\subsection{Benchmark of Characterization Measurements}

Simulations of the characterization measurements were performed with MCNP [55]. A single ${ }^{252} \mathrm{Cf}$ source was simulated in the center of the DDSI instrument cavity at the center of the active length of 
the ${ }^{3} \mathrm{He}$ tubes in the same configuration as the experimental setup. The instrument and lifting mechanism were simulated in detail as shown in Figure 6-5. A void was left for the preamplifiers and cabling in the detector enclosures, however all other critical detector components were simulated. The lead insertions into the detector enclosures were individually modeled and the cadmium lining was modeled as well.
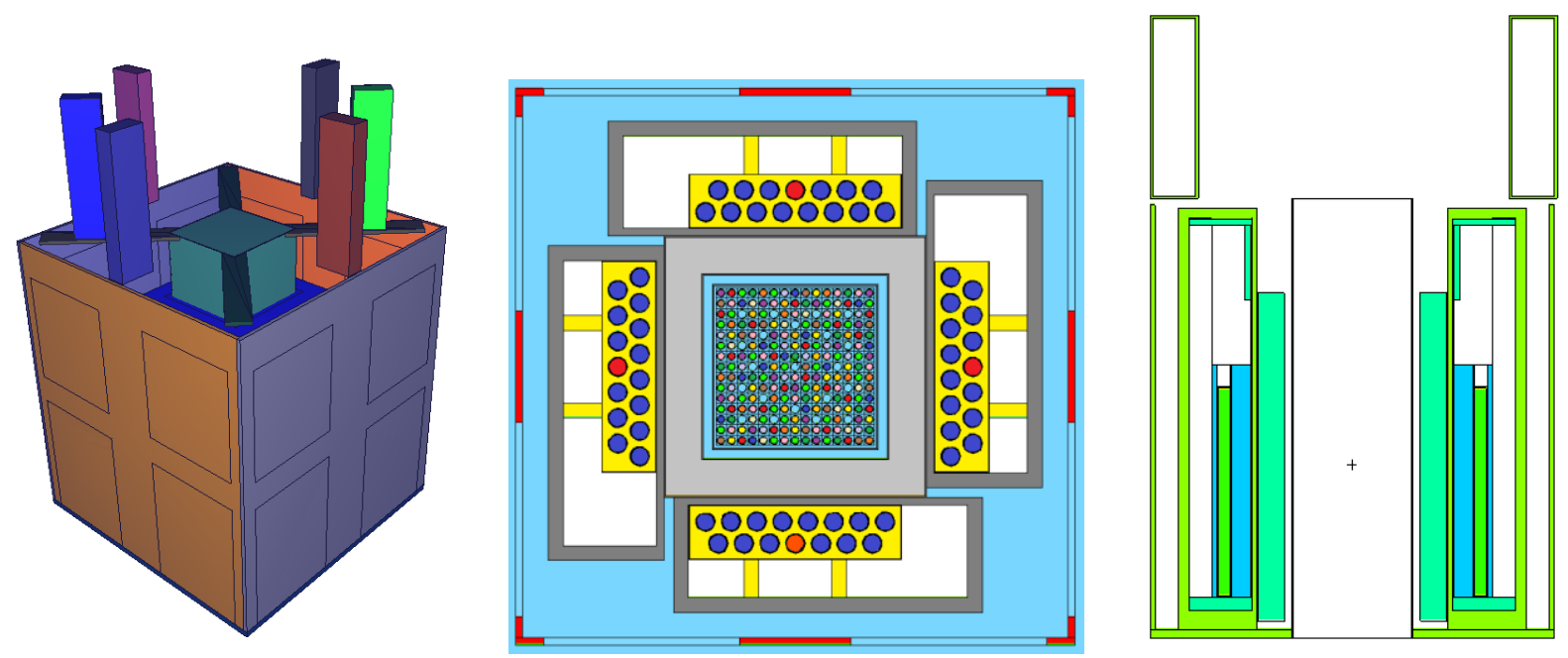

Figure 6-5.3D VisEd DDSI simulation (left), xy plane cross sectional view of DDSI simulation (center), and yz cross sectional view of DDSI simulation (right).

The simulated efficiency with this setup was $11.8 \%$, which is within statistical fluctuation of the experimental deadtime corrected efficiency of $12.0 \% \pm 0.4 \%$. The Rossi-alpha distributions from simulation and experiment also compared well both in shape and magnitude as shown in Figure 6-6, with $1 \sigma$ error bars given on the experimental doubles rates, however they are typically smaller than symbol size. 


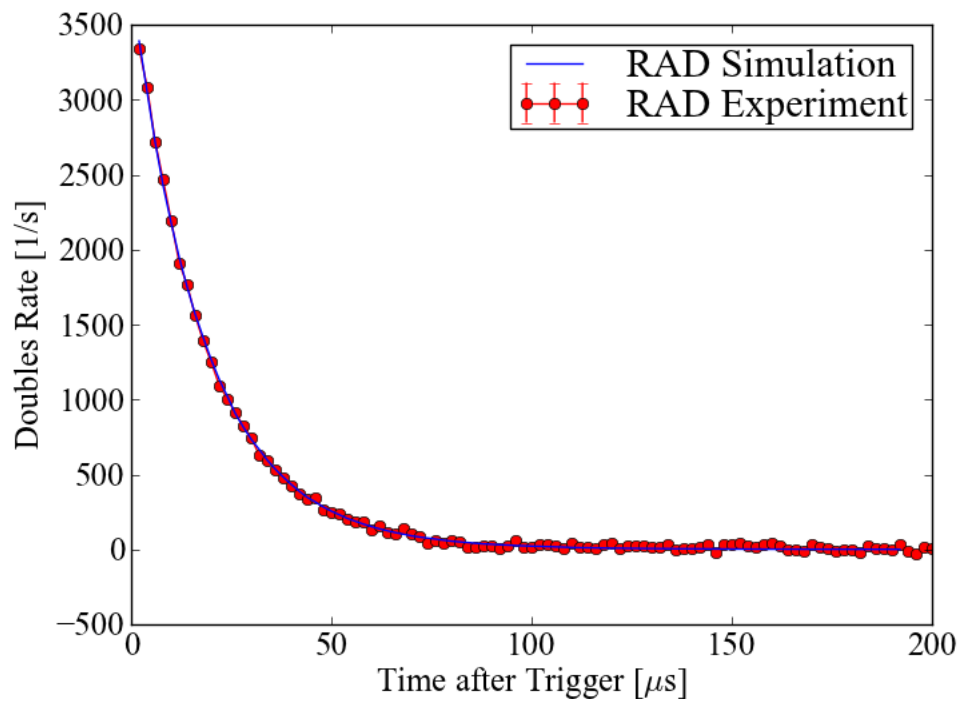

Figure 6-6. Simulated and experimental RADs from a single ${ }^{252} \mathrm{Cf}$ source in the DDSI instrument.

It is useful for evaluation of the accuracy of the model to consider the differences between simulation and experiment on a channel-by-channel basis. Because each channel is considered individually, singles rates are compared rather than doubles rates. The single rates from experiment and simulation for each channel are compared in Figure 6-7.

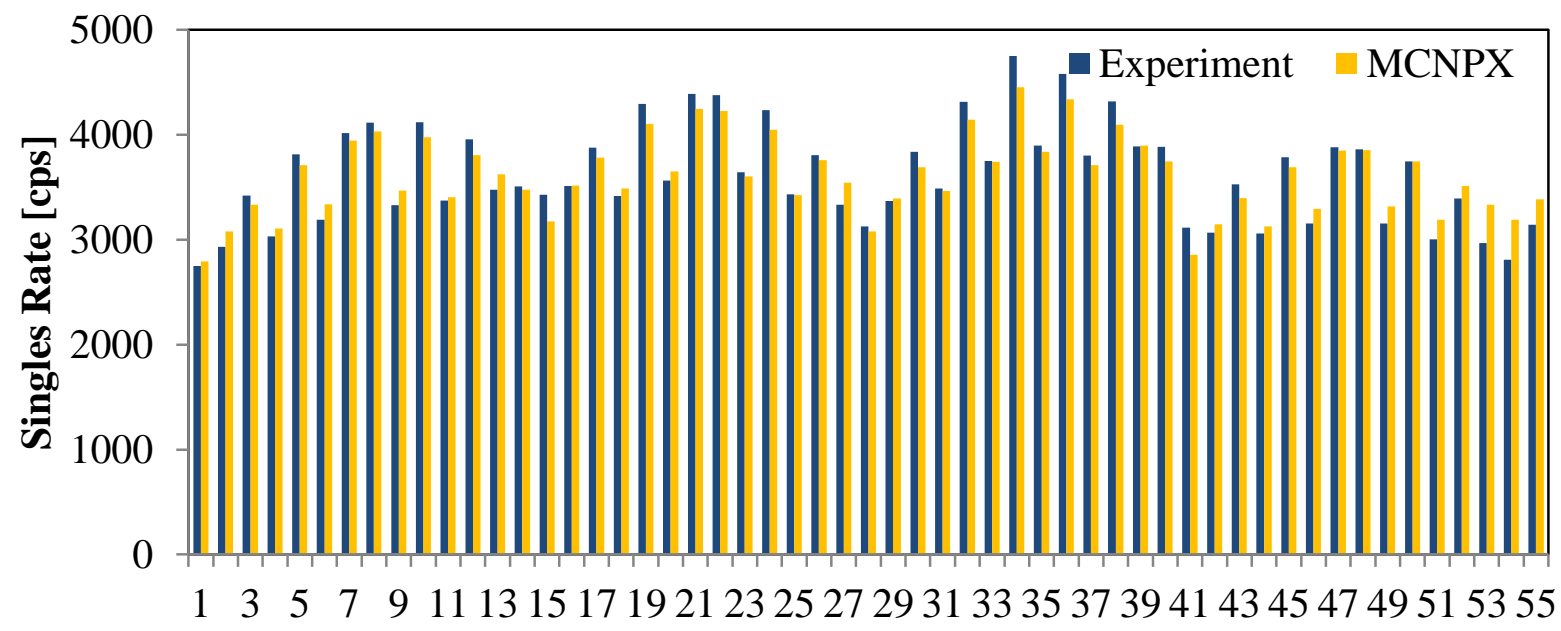

Channel Number

Figure 6-7. Comparison of channel-by-channel singles for experiment and simulation.

Agreement between simulation and experiment on a channel-by-channel basis was very good, indicating an accurate model. Additional measurements were performed in June of 2015 to benchmark a single source in water in the center with and without an empty fuel rack present. 
Though the source was centered as closely as possible in the experiment, it was not exactly in the center. When the source is simulated in the exact center, the channel count rates do not match up as shown in Figure 6-8.

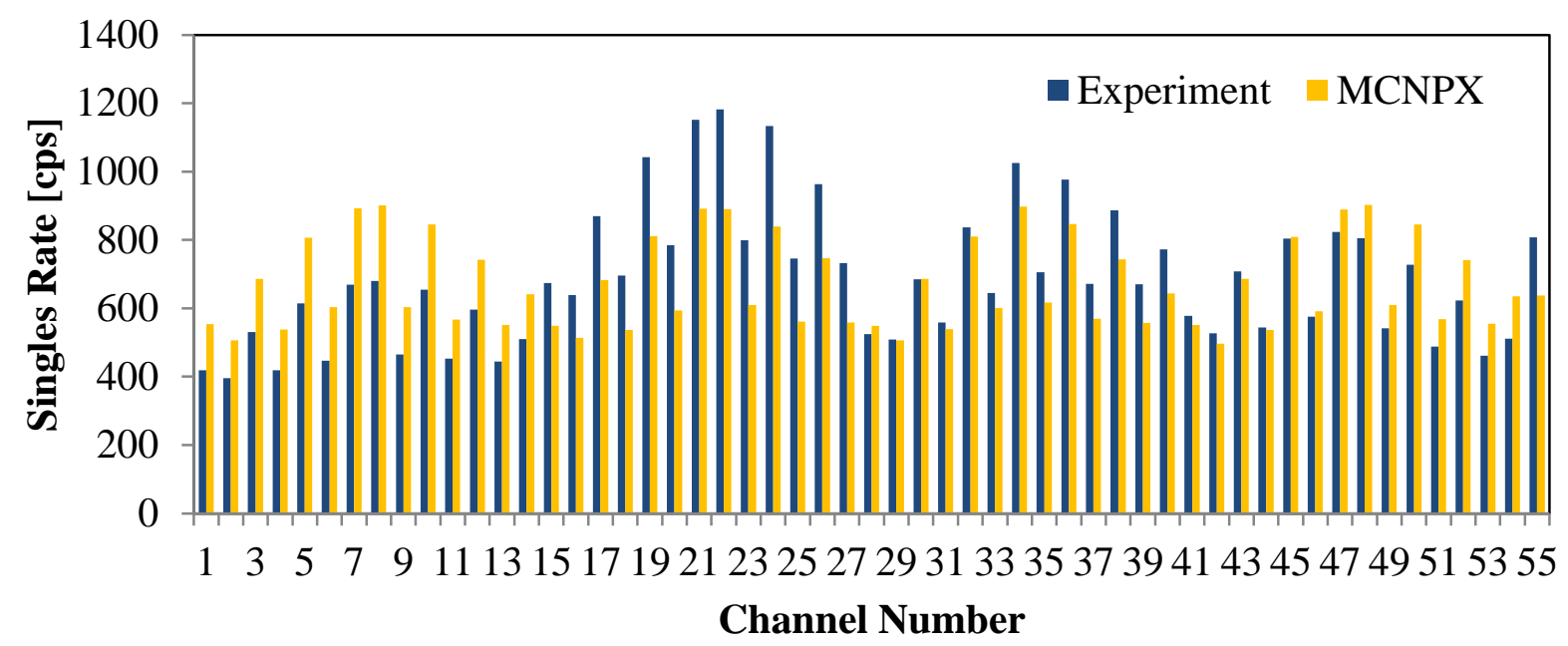

Figure 6-8. Channel-by-channel comparison between experiment and simulation when source is centered in simulation, but likely off-center in experiment.

This figure shows a symmetric distribution in simulation, which is expected because the source is simulated in the exact center of the instrument. In experiment, however, it is clear that there are more counts in channels $15-43$, pods 2 and 3, meaning that the source was shifted slightly to the lower right. Through a series of simulations it was determined that the source was shifted approximately 5 $\mathrm{mm}$ to the right and $13 \mathrm{~mm}$ down. Simulating this shift produces much better agreement as shown in Figure 6-9. The RADs from the same setup are compared in Figure 6-10, with $1 \sigma$ uncertainties given on the experimental data. 


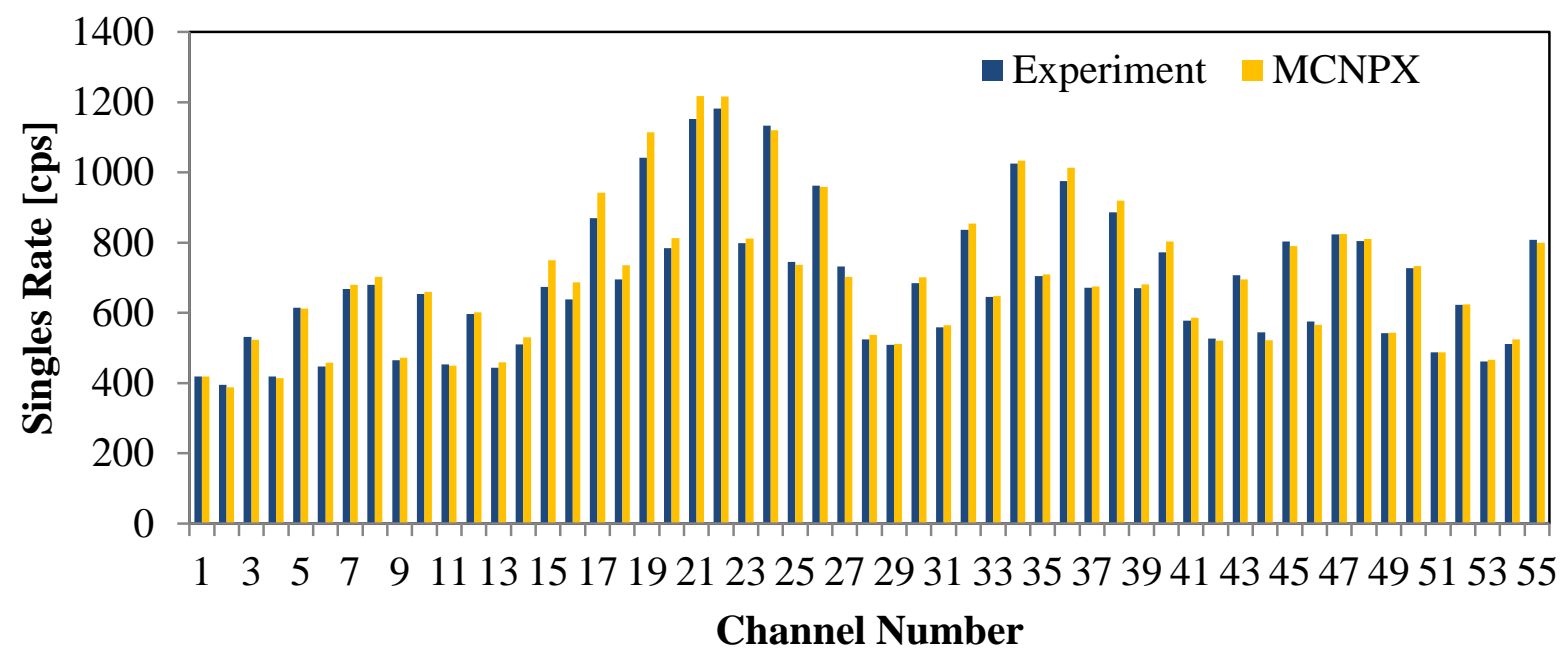

Figure 6-9. Channel-by-channel singles comparison for ${ }^{252} \mathrm{Cf}$ source slightly off-center in water with no fuel rack.

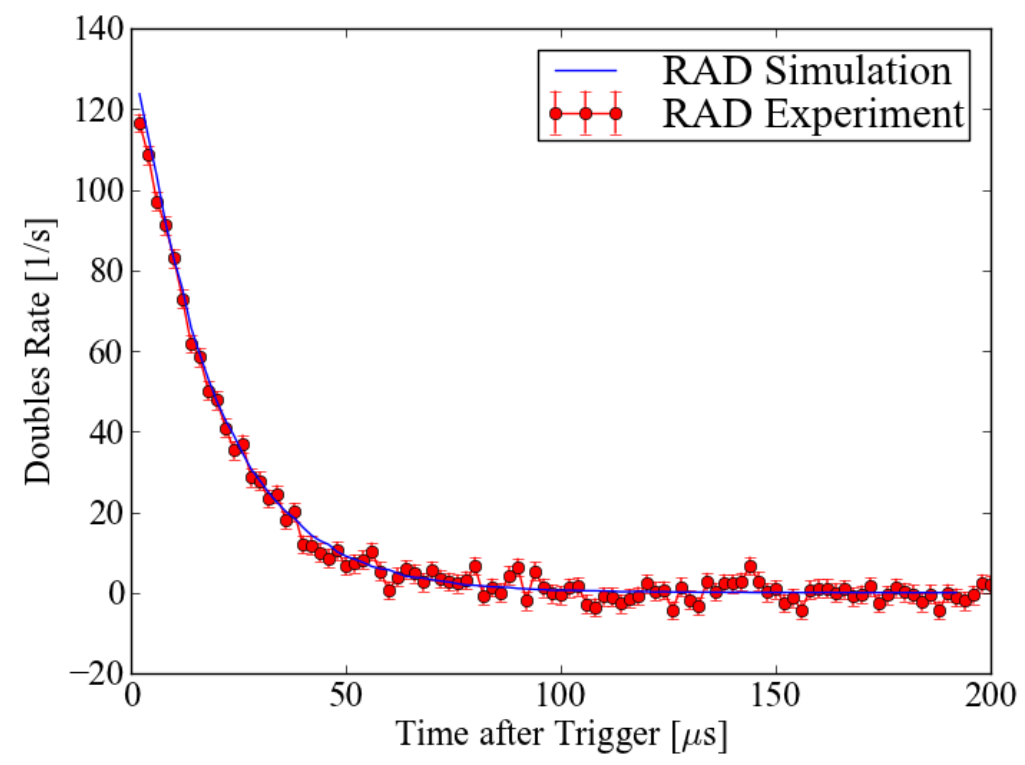

Figure 6-10. Overlain RADs for ${ }^{252} \mathrm{Cf}$ source centered in water with no fuel rack.

The signal is much more sensitive to positioning in water than air because of the large change in the probability of a neutron reaching a detector pod as a function of moderating material in the pathway. By moving the source slightly closer to pods 2 and 3 in the water case the shape of the signal observed in experiment was recreated. This emphasizes the utility of the MCNP simulations in understanding the sometimes unmeasured or unmeasurable details of the experiment. This result also shows that the neutron transport in the submerged DDSI instrument in water is reliable and accurate, and the detector system and measurement setup including the water are being modeled correctly. 
It is also logical to conclude that the model of the source in the center guide tube of the fuel rack would more closely match reality than the model of the source suspended in the water alone. This is because the source location is more constrained when it is confined to the guide tube, and therefore it is easier to replicate in the model. Figure 6-11 and Figure 6-12 show the channel-by-channel comparison and RAD comparison when the source was placed into the center guide tube of the fuel rack.

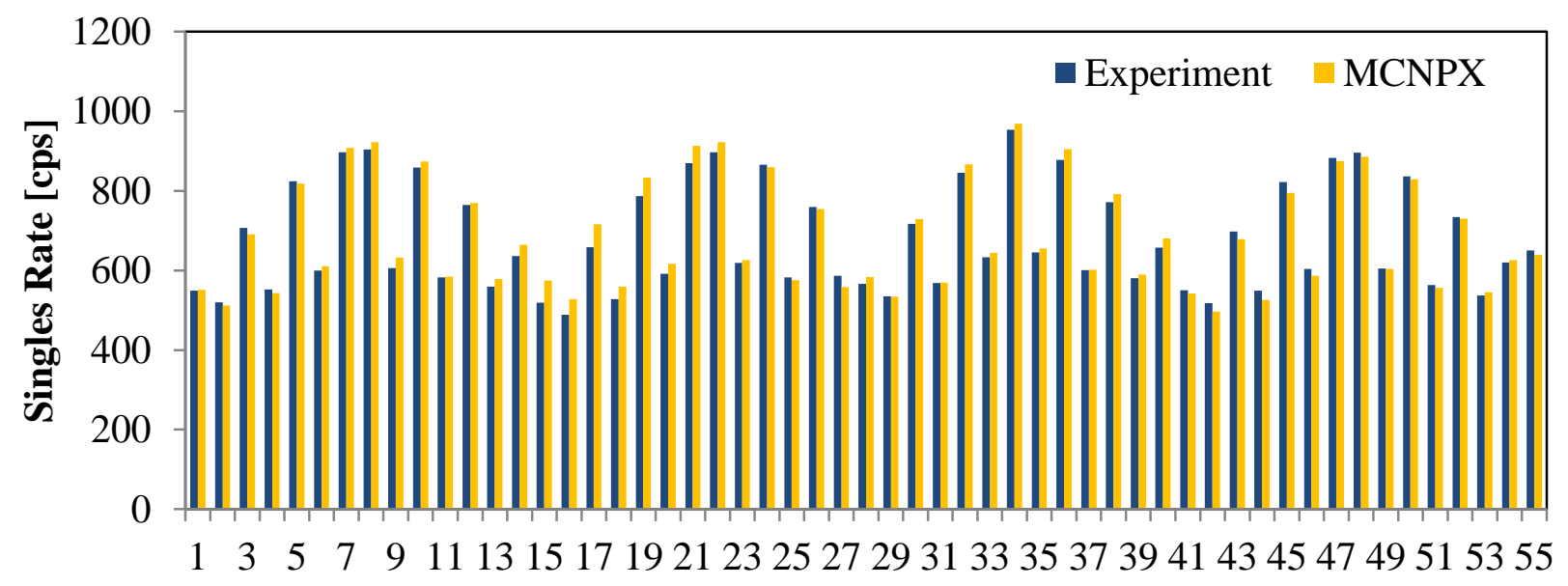

Channel Number

Figure 6-11. Channel-by-channel singles comparison from the measurement of a ${ }^{252} \mathrm{Cf}$ source in the center guide tube of a fuel rack, with the entire instrument submerged in water.

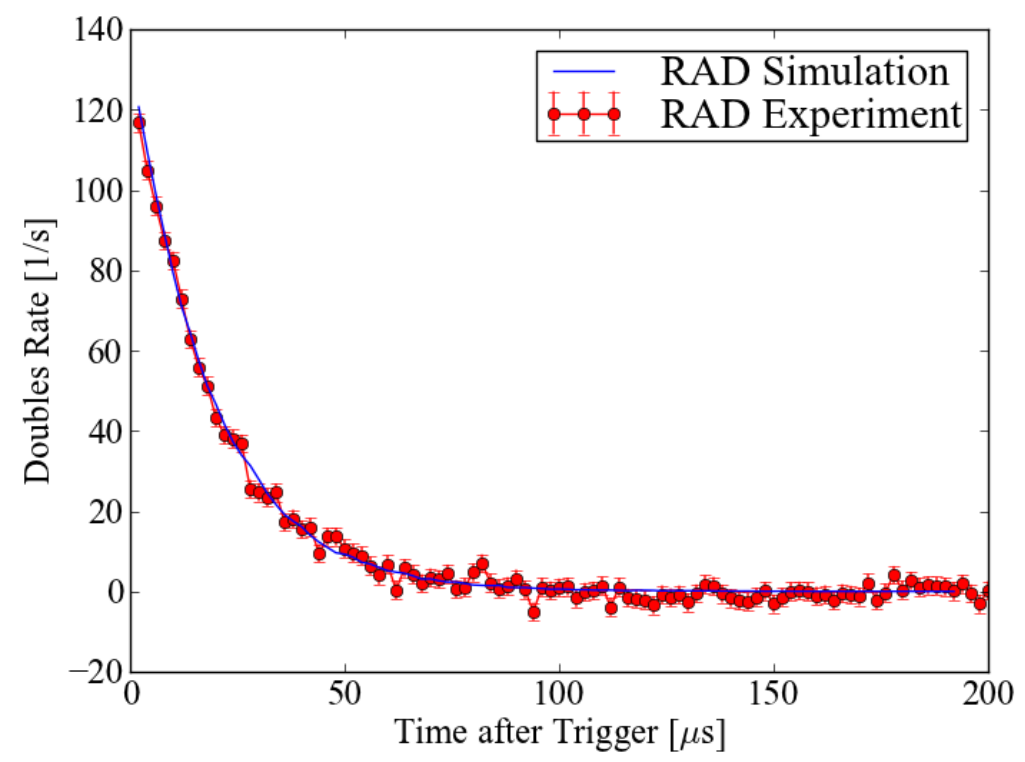

Figure 6-12. Comparison of experimental and simulated RADs for ${ }^{252} \mathrm{Cf}$ source center guide tube in water with fuel rack. 
The channel-by-channel distribution is much more symmetric when the source is centered using the guide tube of the fuel rack, as expected.

\subsection{Neutron Generator and ${ }^{226}$ Ra Measurements}

The four detector enclosures were removed from the DDSI instrument and brought into a shielded cell for high neutron count rate measurements in the presence of a high dose rate gamma ray source. The purpose of these measurements was to create a count rate environment similar to that expected from spent fuel. Though certain physical aspects of spent fuel can be recreated in fresh fuel measurements, the high count rate and high dose rate test must be performed to ensure that the instrument is capable of obtaining accurate neutron count rates in the presence of actual spent fuel.

A D-T neutron generator producing $14 \mathrm{MeV}$ neutrons was used along with a high intensity radium source providing a gamma dose of approximately $20 \mathrm{Rad} / \mathrm{hr}$ to the detectors. The neutron generator was the sole radiation source in the first measurement, and then used in conjunction with the radium in the second measurement. The generator was used at $100 \%$ duty cycle, $80 \mathrm{kV}$, and $70 \mu \mathrm{A}$, and all measurements were 10 minutes long. The generator was placed in the center of the interior space between the pods initially to determine the count rate and ensure that it was not too high to then move the generator closer to the detector pods, which needed to be done in order to later fit the radium source in the center. The measured count rate in this configuration was $6,301,199 \pm 141 \mathrm{cps}$. The generator was then moved to the corner of the setup to leave room for the radium source to be lowered remotely into the center. The measured count rate with the neutron generator alone in the corner was 6,811,439 \pm 145 cps. Figure 6-13 shows the generator in the center of the space between the pods. 


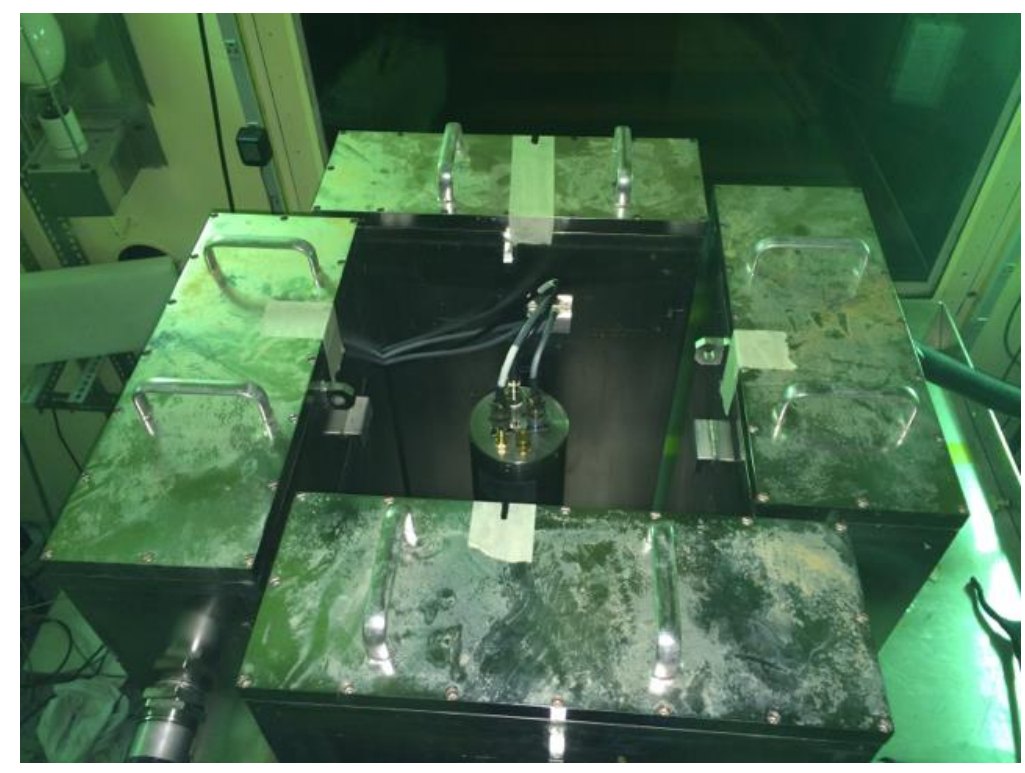

Figure 6-13. Neutron generator centered among DDSI detector pods.

The radium source alone in the center of the setup is equivalent to approximately a $20 \mathrm{R} / \mathrm{hr}$ dose to the ${ }^{3} \mathrm{He}$ tubes. This setup produced a count rate of approximately $1300 \mathrm{cps}$ above background due to gamma pileup. This is negligible compared to the $\sim 7$ million cps count rate of the neutrons at less than $0.02 \%$, and would thus not interfere with the measurement results. To ensure that the gamma dose being applied simultaneously with the neutrons does not interfere with the measured signal, the two sources were measured together. The measured count rate with the radium source in the center and the neutron generator in the corner was $6,720,190 \pm 160 \mathrm{cps}$. Uncertainties are given by INCC and neutron generator uncertainty data $[90,91]$. This equated to a $1.3 \%$ reduction in counts. The drop in count rate can be attributed to the variability in the neutron generator output from shot to shot which has been determined to be approximately 1.4\% [91]. The generator was turned off between the neutron generator alone measurement and the neutron generator and radium measurement. Given that $20 \mathrm{Rad} / \mathrm{hr}$ is the maximum dose ever expected from spent fuel in the DDSI instrument and 7.7 million cps is higher than any neutron count rate ever expected, this represents an extreme case and performance is very good. 


\subsection{Conclusions}

A series of characterization measurements were performed for the DDSI instrument. Single ${ }^{252} \mathrm{Cf}$ sources were measured both in water and air, and instrument parameters including deadtime, dieaway time, and efficiency were determined. These measurements were also used to benchmark the MCNP simulations and agreement between measured and simulated data is within statistical uncertainty. Simulation results were shown to be useful in analyzing details of the experiments and agreement on a channel-by-channel basis proved that the model of the detector instrument and measurement setup were accurate. Finally, the instrument was exposed to extreme neutron and gamma count rates to test for spent fuel scenario behavior and performance was found not to be significantly affected. 


\section{Chapter 7. FreSH NuClEAR FUEL EXPERIMENTS}

As discussed in Section 3.4, fresh fuel can be used as a significantly easier to handle approximation of spent fuel because they share many physical similarities relevant for the DDSI method. The high count rate tests described in the previous chapter demonstrated the capability of the DDSI instrument to measure a spent fuel assembly from an engineering standpoint. The next step is then to test analysis algorithms developed in simulation space and evaluate the capability of the DDSI instrument to characterize spent nuclear fuel, using fresh fuel experiments. For these experiments, 13 different fresh fuel enrichments were created using a combination of depleted uranium (DU), and low-enriched uranium (LEU) fuel rods, as well as LEU rods containing burnable poisons in the form of Gadolinium (Gd). Rossi-alpha distributions were produced from list mode data collected from the different fuel assembly measurements and analyzed using the methods developed for spent fuel and discussed in Chapter 5.

\subsection{Experimental Setup}

The DDSI instrument as described in Section 1.1 was placed in the center of a 500 gallon tank at Los Alamos National Laboratory. Low voltage was applied to power the PDT A-111 preamplifiers

attached to each ${ }^{3} \mathrm{He}$ tube. The data were collected using list mode data acquisition system with a single input from each preamplifier in the instrument. This configuration results in a reduced deadtime of $23 \mathrm{~ns}$ [87]. The data were collected and stored in the form of two files: .dat contains the channel number of each neutron detection and tks contains the corresponding time of detection. The summed signal from all 56 tubes was fed into a JSR-15 shift register as well. List-mode data were converted to RADs with FastTapX RAD production software. For the purpose of this work, we are interested primarily in the early time domain of the RAD from approximately 6-52 $\mu$ s as well as the decomposition of the curve into additive fast and slow components approximated by single 
exponential functions. An empty fuel rack was placed in the center of the instrument as shown in Figure 7-2. Five ${ }^{252} \mathrm{Cf}$ sources totaling approximately $1.4 \mathrm{mCi}$ were suspended in control rod slots distributed both axially and radially to create a more even neutron flux than would be created by placing all sources in the center of the instrument, as seen in Figure 7-2. Two of the sources are weaker than the other three and are thus not as visible in the neutron flux image, however they contribute to spreading the neutron flux more evenly throughout the assembly. Regular tap water was used to fill the tank to a level slightly above the level of the fuel rack. The water served as a moderator of spontaneous fission neutrons from the ${ }^{252} \mathrm{Cf}$ source and induced fission neutrons from ${ }^{235} \mathrm{U}$ in the fuel.

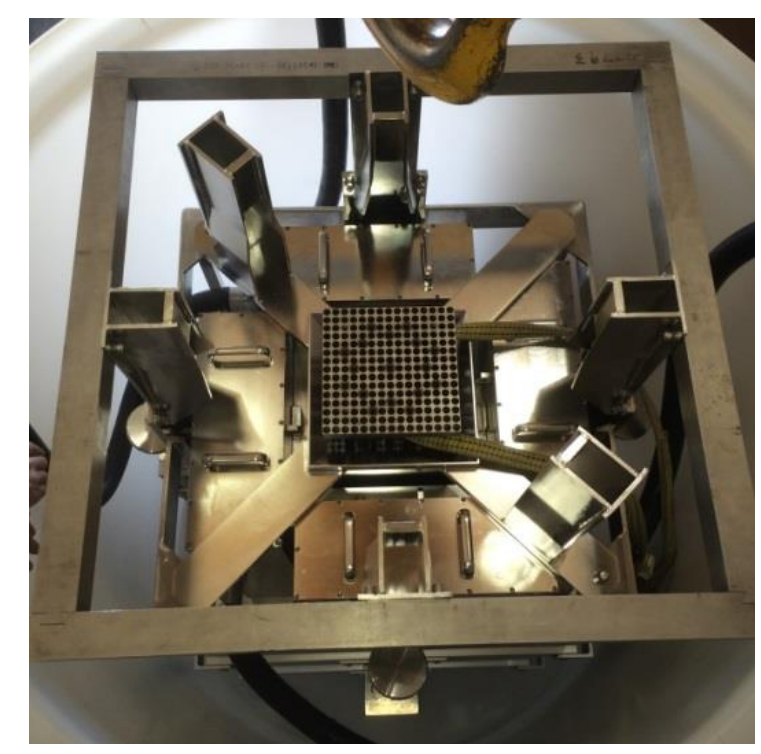

Figure 7-1. Fuel rack centered in DDSI instrument. 

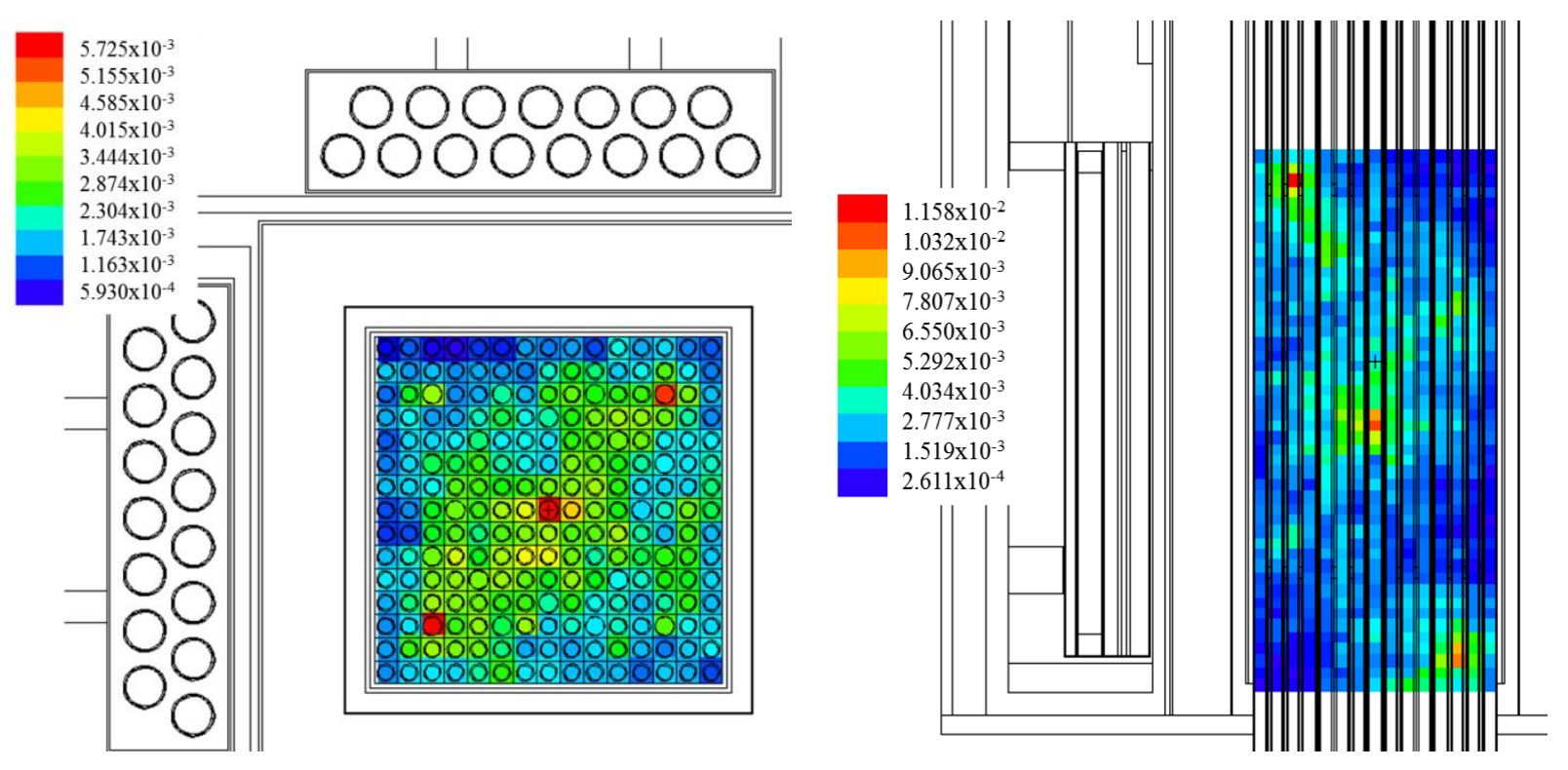

Figure 7-2. Simulated neutron flux from axially and radially distributed ${ }^{252} \mathrm{Cf}$ sources in the fresh fuel assembly.

DU, LEU, and LEU + Gd rods were used to create 13 different symmetric assembly configurations with varying effective enrichments in the fuel rack, as well as 4 different asymmetric configurations. While generally undesirable, asymmetric burnup is common in spent fuel assemblies and therefore considering asymmetric fresh fuel loadings is beneficial for extension to the spent fuel analysis realm. The $\mathrm{LEU}+\mathrm{Gd}$ rods were used in some assemblies to provide varying neutron absorber content as would be the case in spent fuel. The symmetric fuel rod configurations are shown in Figure 7-3, and the asymmetric configurations are shown in Figure 7-4. 


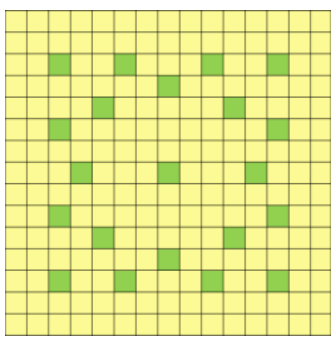

$0.21 \%$

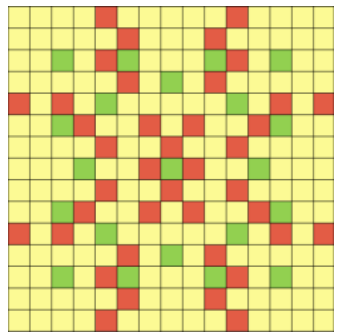

$0.81 \%$

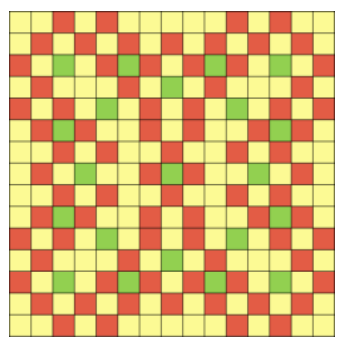

$1.38 \%$

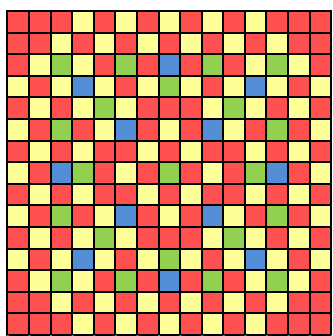

$1.97 \%$

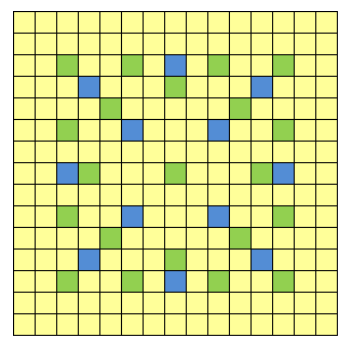

$0.39 \%$

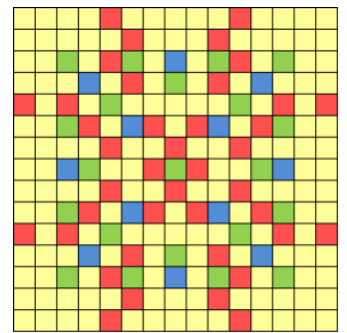

$0.97 \%$

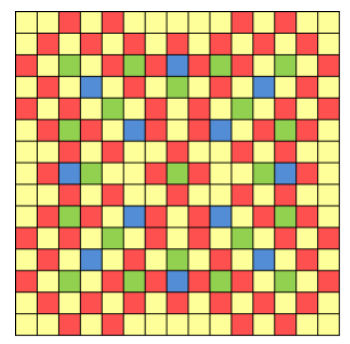

$1.56 \%$

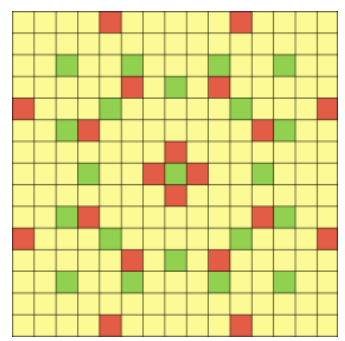

$0.50 \%$

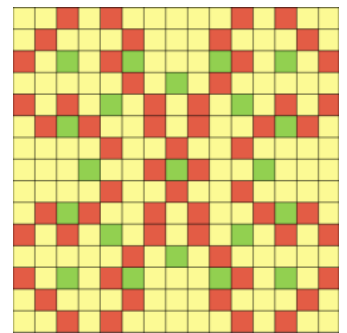

$1.09 \%$

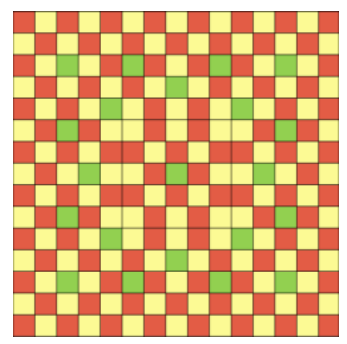

$1.67 \%$

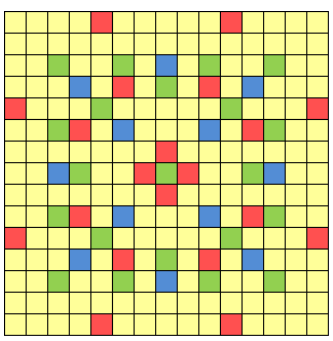

$0.68 \%$

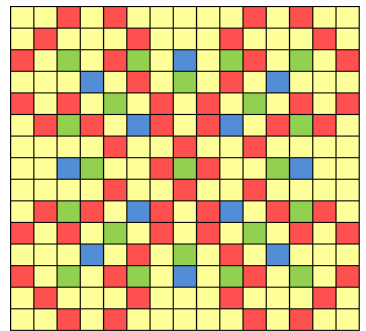

$1.27 \%$

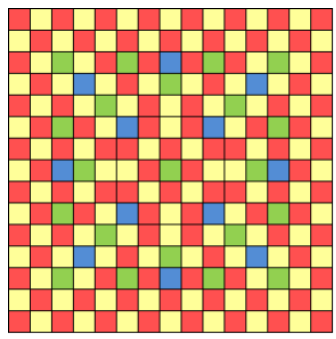

$1.79 \%$

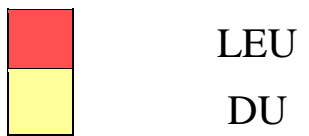

Empty Guide Tubes

Gadolinium

Figure 7-3. Thirteen fuel rod configurations with varying effective enrichments created with LEU, DU, and LEU + gadolinium rods. 


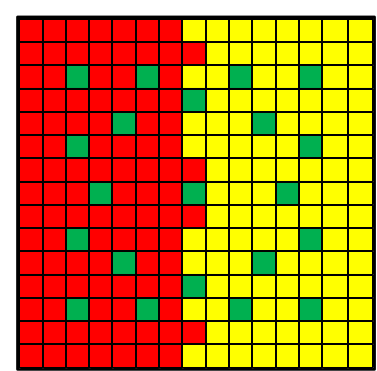

(a)

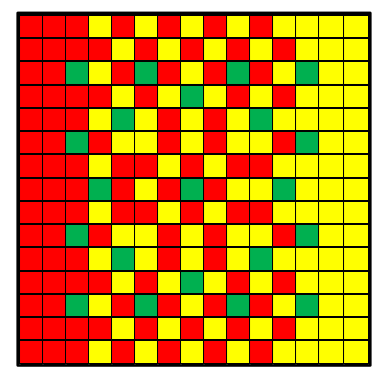

(b)

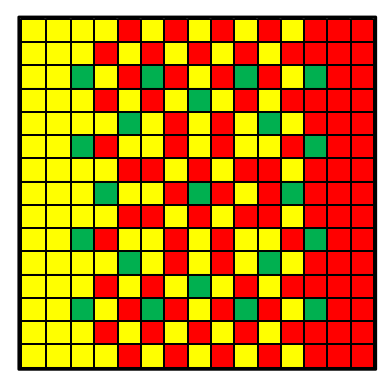

(c)

Figure 7-4. Three different fuel rod configurations with the same effective enrichment but varying degrees of asymmetric distribution of LEU and DU rods. (a) $1.67 \%$ fully asymmetric loading, (b) $1.67 \%$ partially asymmetric loading (b), and (c) reversed $1.67 \%$ partially asymmetric loading.

\subsection{Sensitivity Study Results}

One of the benefits of list-mode data collection for neutron coincidence counting is the ability to consider data on a channel-by-channel basis. Comparing the channel-by-channel data from experiment to that from simulation verifies that the placement of the sources in the model was the same as in the actual experiment as demonstrated in Section 6.5. The DDSI instrument geometry was created in simulation space using mechanical engineer specifications of the instrument in addition to physical dimension measurements taken on-site. The agreement of the characterization measurement results with simulation [87] indicates that the detector model is sufficiently accurate. To compare the experimental and simulated data for each channel, the 10 minute measurement of five sources in the otherwise empty fuel rack in water was compared with simulation of the same setup. Singles in each channel from experiment and simulation are shown in Figure 7-5. Four fewer channels were used in this set of experiments than in the characterization measurements of Chapter 6 because of performance problems. Two preamplifiers were displaying gain drift and were thus omitted, and two detectors lost signal due to cabling issues. 


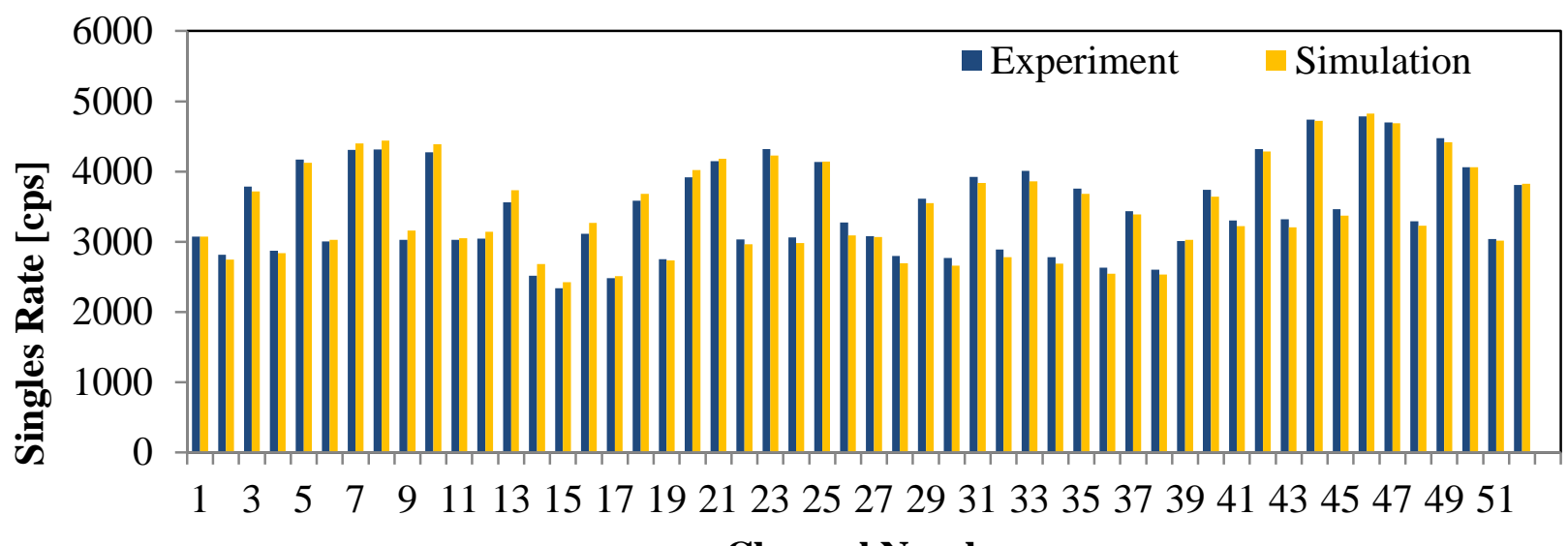

Channel Number

Figure 7-5. Channel-by-channel comparison of experimental and simulated data for base case of five ${ }^{252} \mathrm{Cf}$ sources in the otherwise empty fuel rack in water.

The trends of the experimental channel-by-channel data are closely matched by the simulated data, indicating that the placement of the fuel rack and sources in the instrument in simulation space can be considered identical. This also indicates that the DDSI instrument is being modeled correctly.

The simulated singles count rates were obtained with an infinite gate F8 capture tally in MCNP [58]. The doubles count rates were obtained both in experiment and simulation from a $24 \mu$ s gate after a 4 $\mu$ s pre-delay in the RAD. The simulated RAD was produced with the F8 capture tally method as described in [8] and Section 4.1.2 of this thesis. The experimental RAD was produced with the FastTapX software package. Because good agreement was obtained between the experimental and simulated base case of five ${ }^{252} \mathrm{Cf}$ sources in water and single sources in air (Section 6.5), it was concluded that the model of the detector system was correct. However when fresh fuel was introduced, the agreement began to diverge, as will be shown in the end of this section. Extensive sensitivity analysis was done on different aspects of the model to improve its agreement with experiment in the fuel measurement cases without affecting the base case significantly.

\subsubsection{Water Gap}

Though mechanical engineer drawings were used to exactly simulate the instrument, it was found after assembly that the detector enclosures are slightly tilted with respect to the lead shield, resulting in a small gap of approximately $5 \mathrm{~mm}$ between the shield and the pod. The exact size of this gap during measurements was unknown due to the placement of the instrument in the water tank. The expected gap size of $5 \mathrm{~mm}$ was used in the base case. This gap size also provided good agreement in 
the characterization measurements benchmark [87]. The base case gap size is compared with results from plus or minus $1 \mathrm{~mm}$ of water in the gap in Table 7-I.

Table 7-I. Comparison of experiment with simulations in which the water gap varies from 4 to $6 \mathrm{~mm}$.

\begin{tabular}{lllll}
\hline & $\mathbf{0 . 2 1 \%}$ Singles & $\mathbf{0 . 2 1 \%}$ Doubles & $\mathbf{1 . 9 7 \%}$ Singles & $\mathbf{1 . 9 7 \%}$ Doubles \\
\hline Experiment & $340799 \pm 341$ & $15602 \pm 101$ & $598396 \pm 60$ & $48772 \pm 176$ \\
Base & 338642 & 16163 & 627210 & 55735 \\
$\mathbf{+ 1} \mathbf{~ m m ~ H} \mathbf{O}$ & 329757 & 15364 & 610917 & 52862 \\
$\mathbf{- 1} \mathbf{~ m m ~ H} \mathbf{O}$ & 348164 & 17096 & 643747 & 58711 \\
\hline
\end{tabular}

Though the doubles and singles rates are reduced with a larger water gap bringing the results closer to what was observed in experiment, a significant gap would be needed to make the results match, and that would cause a large disagreement in the base case.

\subsubsection{Neutron Absorbers}

The simulated singles and doubles results scaled in deviation from experimental results in proportion to assembly enrichment, i.e. higher enrichment assemblies had the worse agreement between simulation and experiment and lower enrichment assemblies had the best agreement. This indicates a problem with multiplication in the model because as enrichment increases, neutrons are multiplying more in the simulation than in the experiment. The neutron absorber content in the system affects multiplication by removing neutrons from the system that would otherwise go on to cause fission or be detected. This can be tested by adding neutron absorbers to the simulated system. Neutron absorber content was altered by adding boron to the water and cadmium to the lead in small amounts to analyze the effect on singles and doubles agreement in the models. The base case contains no added absorbers. The results are compared in Table 7-II. 
Table 7-II. Comparison of experiment with base case and added neutron absorber models for highest and lowest enrichment cases. Each absorber is added independently.

\begin{tabular}{lllll}
\hline & $\mathbf{0 . 2 1 \%}$ Singles & $\mathbf{0 . 2 1 \%}$ Doubles & $\mathbf{1 . 9 7 \%}$ Singles & $\mathbf{1 . 9 7 \%}$ Doubles \\
\hline Experiment & $340799 \pm 341$ & $15602 \pm 101$ & $598396 \pm 60$ & $48772 \pm 176$ \\
Base & 338642 & 16163 & 627210 & 55735 \\
$\mathbf{+ 1 0 0}$ ppm B & 331091 & 15831 & 591578 & 51304 \\
$\mathbf{+ 1 5 0}$ ppm B & 327805 & 15653 & 576610 & 49453 \\
$\mathbf{+} \mathbf{2 0 0}$ ppm B & 324906 & 15492 & 562784 & 47808 \\
\hline $\mathbf{1 0 0}$ ppm Cd & 338180 & 16106 & 622030 & 55180 \\
\hline
\end{tabular}

The addition of neutron absorbers results in the simulation behaving as expected by showing a large reduction in singles and doubles in the high enrichment case and a small reduction in the low enrichment case. $200 \mathrm{ppm} \mathrm{B}$ brings the count rates down to be nearly equivalent with the experimental results, however later testing of the experiment tank water with mass spectrometry showed that there was no sizable amount of boron in the water. The cadmium did not make a large difference in the simulated results. There could alternatively be boron or larger amounts of $\mathrm{Cd}$ in the lead, but that cannot be confirmed because the lead can no longer be tested now that it has been encased in stainless steel.

\subsubsection{Data Libraries}

Different cross section libraries were used in MCNP to determine whether changes in cross sections used affected the singles and doubles results. Fission models were also varied to see whether the default sampled nubar values for fissile and spontaneous fission isotopes or the LLNL fission model for neutron-induced and spontaneous fission nubar values produced results that more closely matched experiment. The default fission treatment and the .70c libraries are used in the base case. The .80c libraries and LLNL fission model are compared to the base case in Table 7-III. 
Table 7-III. Comparison of data library options to defaults in base case for highest and lowest enrichments.

\begin{tabular}{lllll}
\hline & $\mathbf{0 . 2 1 \%}$ Singles & $\mathbf{0 . 2 1 \%}$ Doubles & $\mathbf{1 . 9 7 \%}$ Singles & $\mathbf{1 . 9 7 \%}$ Doubles \\
\hline Experiment & $340799 \pm 341$ & $15602 \pm 101$ & $598396 \pm 60$ & $48772 \pm 176$ \\
Base & 338642 & 16163 & 627210 & 55735 \\
LLNL Fission & 339138 & 16143 & 626981 & 54805 \\
.80c data & 340134 & 16229 & 628428 & 55795 \\
\hline
\end{tabular}

The LLNL fission model produced results that more closely matched the experimental results, however it did not change the results enough to be considered a solution to the simulation and experiment disagreement. The .80c libraries made very little difference.

\subsubsection{Water Temperature}

The fresh fuel measurements were conducted in two different campaigns, one during a cold spell in May 2015 and one when the weather was considerably warmer in the following month. The effect of temperature (and therefore density) of the water on the count rates was evaluated and results are given in Table 7-IV. The temperature used in the base case was $68^{\circ} \mathrm{F}$.

Table 7-IV. Effect of water temperature on highest and lowest enrichment cases.

\begin{tabular}{lllll}
\hline & $\mathbf{0 . 2 1 \%}$ Singles & $\mathbf{0 . 2 1 \%}$ Doubles & $\mathbf{1 . 9 7 \%}$ Singles & $\mathbf{1 . 9 7 \%}$ Doubles \\
\hline Experiment & $340799 \pm 341$ & $15602 \pm 101$ & $598396 \pm 60$ & $48772 \pm 176$ \\
Base $\left(\mathbf{6 8}^{\mathbf{0}} \mathbf{F}\right)$ & 338642 & 16163 & 627210 & 55735 \\
$\mathbf{7 8}^{\mathbf{T}} \mathbf{F}$ & 339245 & 16213 & 627652 & 55794 \\
\hline
\end{tabular}

The temperature of the water was not found to make any significant difference in the results.

\subsubsection{Conclusions of the Sensitivity Study}

The only parameters found to make a considerable difference in the simulated results were the width of the water gap and large amounts of boron in the water. Enlarging the gap to create agreement between simulation and experiment for the highest enriched cases would create disagreement in the base case of 5 sources in an empty fuel rack, and therefore would not be accurate. The boron impurity was not found in the water after sampling, however, boron or large amounts of cadmium 
could possibly be in the lead. This is a very unlikely scenario given the professional construction of the lead shield, however.

\subsection{Fresh Fuel Measurement Results}

Because none of the parameters in the sensitivity study brought the simulated fuel loading experiments into agreement with the experimental data collected in the May measurement campaign, the symmetric and asymmetric fuel loading experiments were repeated in October of 2015. Between the two experimental campaigns, 2 channels had to be excluded due to preamplifier failure, and the ${ }^{252} \mathrm{Cf}$ sources naturally decayed, and thus the count rates were expected to be lower in the second set of measurements. To check for consistency between the experiments in May and October we can take a ratio of singles and doubles for each fuel assembly and look for a consistent ratio between the different loadings. These results are presented in Figure 7-6.
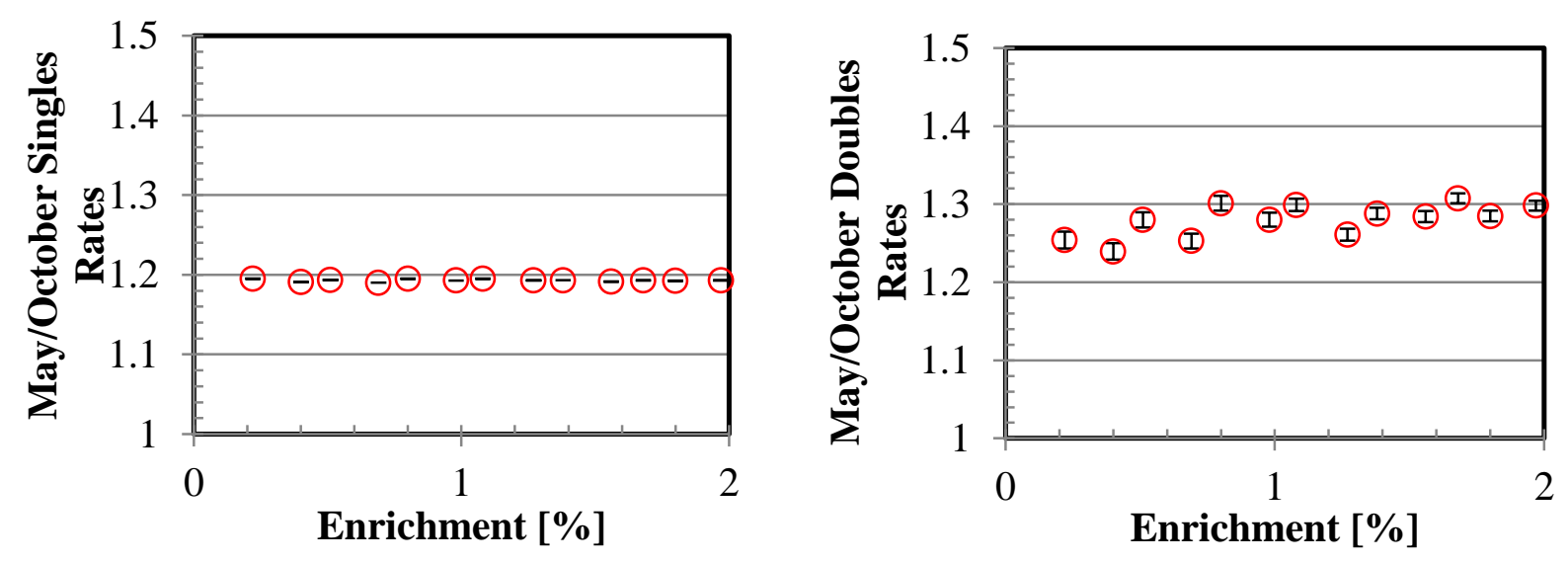

Figure 7-6. Ratio of May/October singles and doubles rates.

The ratio is consistent for singles and doubles, indicating that the measurements were repeatable. There was more fluctuation in the doubles ratios because the statistics are not as good on doubles as singles.

Experimental and simulated singles and doubles data from each symmetric and asymmetric fuel rod configuration are compared in Table $7-\mathrm{V}$ in addition to the five ${ }^{252} \mathrm{Cf}$ sources base case $\left[{ }^{92}\right]$. Agreement is good in the low count rate cases, however it strays in the higher count rate cases and singles disagreement is as high as $7 \%$ while doubles disagreement is as high as $19 \%$. 
The fuel pins were modeled according to a series of $\mathrm{x}$-ray transmission measurements performed in 2013 [93], however the uncertainty on the exact specifications of the fuel rods in the measurements is high, on the order of 50\% in many cases. Therefore the original manufacturer specifications are used for the enrichments in the model. The DU enrichment is $0.21 \%$, the LEU enrichment is $3.19 \%$, and the average Gd rod enrichment is $3.27 \%$. Density values for the rods are not precisely known and were obtained from measurement data from [93]. In reality the fuel pins may vary in density because the pellets are pushed together with springs at the end of the fuel rods, however these springs may wear over time and result in more air between pellets. The average density is modeled for each pin in MCNP, which could result in artificially high multiplication in simulations if the simulated density is too high. For example, a $0.2 \mathrm{~g} / \mathrm{cm}^{2}$ change in density results in a $\sim 8 \%$ change in the doubles rate. Previous benchmarks of active interrogation of the LANL fresh fuel rods have had disagreement between simulation and experiment as well [94], and the cause for the disagreement was ultimately undetermined. There has also been extensive work done identifying nuclear data issues leading to disagreement, particularly with nubar values [95,96]; however this area of study requires much more data and experimental work to confirm the correct values. The trend with multiplication could also imply a deadtime correction problem with the experimental data, however the deadtime for the system is low as discussed in Section 6.3. A combination of these issues could lead to disagreement of this magnitude in the high multiplication assembly simulations, and therefore it is determined that the model is as accurate as it can be with the information available for the construction of this model. 
Table 7-V. Comparison of the singles and doubles rates from October experiments and simulation for 13 symmetric enrichments, and 3 asymmetric loadings of $1.59 \%$ enrichment. Experimental data have been dead-time corrected.

\begin{tabular}{|c|c|c|c|c|c|c|}
\hline & \multicolumn{3}{|c|}{ Singles } & \multicolumn{3}{|c|}{ Doubles (4 $\mu \mathrm{s}$ PD, $24 \mu \mathrm{s}$ GW) } \\
\hline Enrichment & Experiment & MCNP & $\begin{array}{c}\% \\
\text { Diff. }\end{array}$ & Experiment & MCNP & $\begin{array}{c}\% \\
\text { Diff. }\end{array}$ \\
\hline 5 Sources Alone & $\begin{array}{c}180,601 \pm \\
0.01 \%\end{array}$ & $\begin{array}{c}179,581 \pm \\
0.01 \%\end{array}$ & $-1 \%$ & $\begin{array}{c}5,575 \pm \\
0.7 \%\end{array}$ & $\begin{array}{c}5,738 \pm \\
0.7 \%\end{array}$ & $3 \%$ \\
\hline $0.21 \%$ & $\begin{array}{c}285,215 \pm \\
0.01 \%\end{array}$ & $\begin{array}{c}285,398 \pm \\
0.01 \%\end{array}$ & $0 \%$ & $\begin{array}{c}12,442 \pm \\
0.6 \%\end{array}$ & $\begin{array}{c}12,865 \pm \\
0.5 \%\end{array}$ & $3 \%$ \\
\hline $0.39 \%$ & $\begin{array}{c}273,790 \pm \\
0.01 \%\end{array}$ & $\begin{array}{c}272,037 \pm \\
0.01 \%\end{array}$ & $-1 \%$ & $\begin{array}{c}12,256 \pm \\
0.5 \%\end{array}$ & $\begin{array}{c}12,379 \pm \\
0.5 \%\end{array}$ & $1 \%$ \\
\hline $0.50 \%$ & $\begin{array}{c}334,596 \pm \\
0.01 \%\end{array}$ & $\begin{array}{c}337,454 \pm \\
0.01 \%\end{array}$ & $1 \%$ & $\begin{array}{l}15,872 \pm \\
0.5 \%\end{array}$ & $\begin{array}{c}16,829 \pm \\
0.5 \%\end{array}$ & $6 \%$ \\
\hline $0.68 \%$ & $\begin{array}{c}310,614 \pm \\
0.01 \%\end{array}$ & $\begin{array}{c}310,110 \pm \\
0.01 \%\end{array}$ & $0 \%$ & $\begin{array}{c}15,067 \pm \\
0.5 \%\end{array}$ & $\begin{array}{c}15,531 \pm \\
0.5 \%\end{array}$ & $3 \%$ \\
\hline $0.81 \%$ & $\begin{array}{c}385,936 \pm \\
0.01 \%\end{array}$ & $\begin{array}{c}393,049 \pm \\
0.01 \%\end{array}$ & $3 \%$ & $\begin{array}{c}19,928 \pm \\
0.5 \%\end{array}$ & $\begin{array}{c}22,022 \pm \\
0.4 \%\end{array}$ & $11 \%$ \\
\hline $0.97 \%$ & $\begin{array}{c}345,743 \pm \\
0.01 \%\end{array}$ & $\begin{array}{c}347,431 \pm \\
0.01 \%\end{array}$ & $1 \%$ & $\begin{array}{c}17,945 \pm \\
0.5 \%\end{array}$ & $\begin{array}{c}19,228 \pm \\
0.4 \%\end{array}$ & $7 \%$ \\
\hline $1.09 \%$ & $\begin{array}{c}445,748 \pm \\
0.01 \%\end{array}$ & $\begin{array}{c}457,731 \pm \\
0.01 \%\end{array}$ & $4 \%$ & $\begin{array}{c}26,194 \pm \\
0.4 \%\end{array}$ & $\begin{array}{c}29,532 \pm \\
0.4 \%\end{array}$ & $13 \%$ \\
\hline $1.27 \%$ & $\begin{array}{c}390,310 \pm \\
0.01 \%\end{array}$ & $\begin{array}{c}395,020 \pm \\
0.01 \%\end{array}$ & $2 \%$ & $\begin{array}{c}23,047 \pm \\
0.4 \%\end{array}$ & $\begin{array}{c}24,652 \pm \\
0.4 \%\end{array}$ & $7 \%$ \\
\hline $1.38 \%$ & $\begin{array}{c}511,061 \pm \\
0.01 \%\end{array}$ & $\begin{array}{c}528,952 \pm \\
0.005 \%\end{array}$ & $5 \%$ & $\begin{array}{c}34,139 \pm \\
0.4 \%\end{array}$ & $\begin{array}{c}39,337 \pm \\
0.3 \%\end{array}$ & $15 \%$ \\
\hline $1.56 \%$ & $\begin{array}{c}433,541 \pm \\
0.01 \%\end{array}$ & $\begin{array}{c}440,068 \pm \\
0.005 \%\end{array}$ & $3 \%$ & $\begin{array}{c}28,052 \pm \\
0.4 \%\end{array}$ & $\begin{array}{c}30,663 \pm \\
0.3 \%\end{array}$ & $9 \%$ \\
\hline $1.67 \%$ & $\begin{array}{c}586,784 \pm \\
0.01 \%\end{array}$ & $\begin{array}{c}611,214 \pm \\
0.004 \%\end{array}$ & $6 \%$ & $\begin{array}{c}44,380 \pm \\
0.3 \%\end{array}$ & $\begin{array}{c}52,799 \pm \\
0.3 \%\end{array}$ & $19 \%$ \\
\hline $1.79 \%$ & $\begin{array}{c}475,468 \pm \\
0.01 \%\end{array}$ & $\begin{array}{c}483,923 \pm \\
0.005 \%\end{array}$ & $3 \%$ & $\begin{array}{c}34,497 \pm \\
0.3 \%\end{array}$ & $\begin{array}{c}37,297 \pm \\
0.3 \%\end{array}$ & $11 \%$ \\
\hline $1.97 \%$ & $\begin{array}{c}501,613 \pm \\
0.01 \%\end{array}$ & $\begin{array}{c}511,060 \pm \\
0.005 \%\end{array}$ & $4 \%$ & $\begin{array}{c}37,569 \pm \\
0.3 \%\end{array}$ & $\begin{array}{c}42,085 \pm \\
0.3 \%\end{array}$ & $12 \%$ \\
\hline $\begin{array}{l}1.67 \%, \text { full } \\
\text { asymmetry }\end{array}$ & $\begin{array}{c}520,480 \pm \\
0.01 \%\end{array}$ & $\begin{array}{c}540,965 \pm \\
0.005 \%\end{array}$ & $5 \%$ & $\begin{array}{c}41,974 \pm \\
0.3 \%\end{array}$ & $\begin{array}{c}49,267 \pm \\
0.3 \%\end{array}$ & $17 \%$ \\
\hline $\begin{array}{l}1.67 \%, \text { partial } \\
\text { asymmetry }\end{array}$ & $\begin{array}{c}561,063 \pm \\
0.01 \%\end{array}$ & $\begin{array}{c}583,613 \pm \\
0.005 \%\end{array}$ & $6 \%$ & $\begin{array}{c}43,105 \pm \\
0.3 \%\end{array}$ & $\begin{array}{c}51,455 \pm \\
0.3 \%\end{array}$ & $19 \%$ \\
\hline $\begin{array}{c}1.67 \%, \text { partial } \\
\text { asymmetry, reversed }\end{array}$ & $\begin{array}{c}555,648 \pm \\
0.01 \%\end{array}$ & $\begin{array}{c}581,977 \pm \\
0.005 \%\end{array}$ & $7 \%$ & $\begin{array}{c}42,158 \pm \\
0.3 \%\end{array}$ & $\begin{array}{c}49,994 \pm \\
0.3 \%\end{array}$ & $19 \%$ \\
\hline
\end{tabular}

In addition to comparing the singles and doubles rates, it is informative to compare the die-away times from the RAD fitting as well. These values were obtained with a series of curve-fitting algorithms implemented in Python [89] and described in detail in Section 2.4. Identical RAD curve 
analysis was performed on experimentally obtained list mode RAD data and simulated RAD data. The fitting procedure first obtained a single exponential fit using a non-linear least squares algorithm for the 80-150 $\mu$ s range of the RAD. This curve, called the slow component, was extended back to 0 $\mu$ s and the values were subtracted from the RAD data. The remaining data were again fit by a single exponential and this curve was called the fast component. Finally, the data in the 6-52 $\mu$ s range of the RAD were fit with a single exponential and the die-away time from that time domain was called the early die-away time. Figure 7-7 thru Figure 7-14 show comparisons between experimental and simulated RADs and exponential fits for four different enrichment cases representing the lowest, highest and two middle enrichment assemblies.
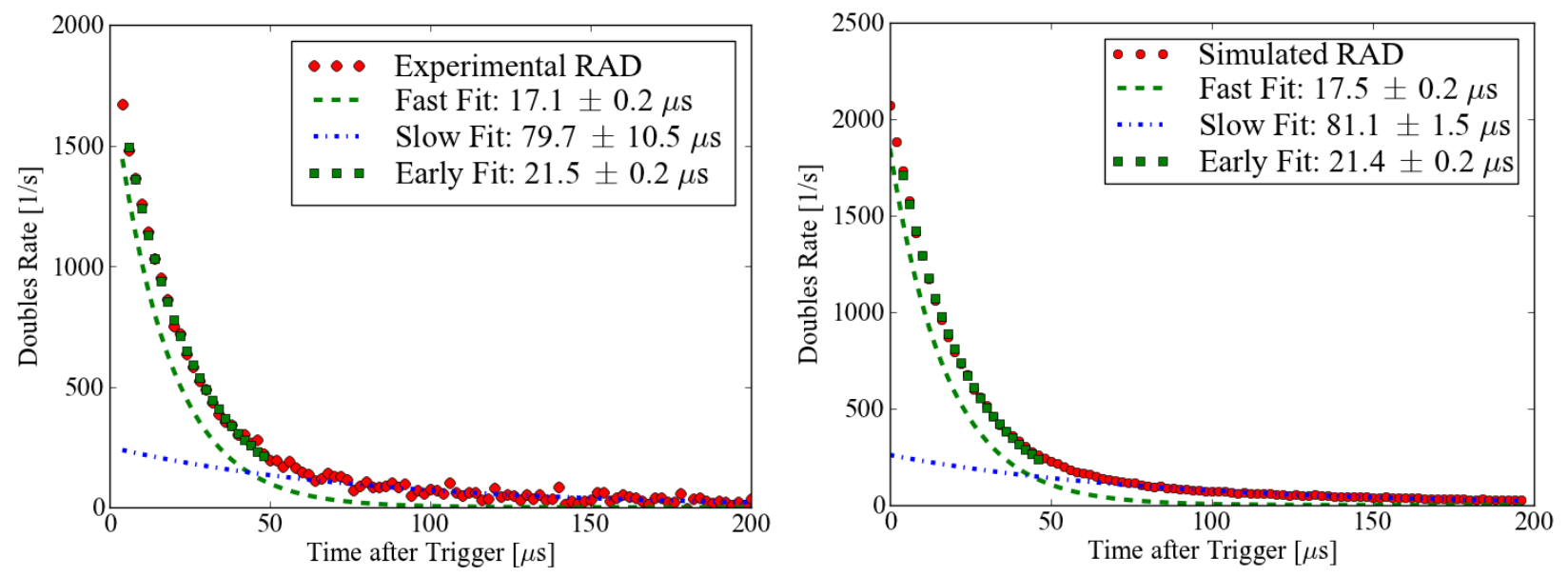

Figure 7-7. Comparison of die-away times of experimental and simulated RADs from $0.21 \%$ enrichment case.

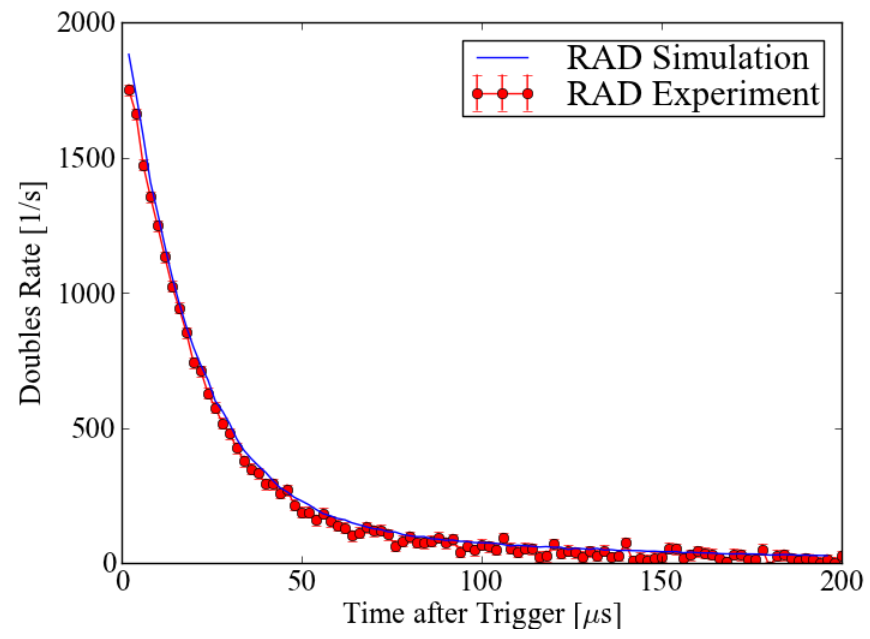

Figure 7-8. Overlain RADs from $0.21 \%$ enrichment case. 

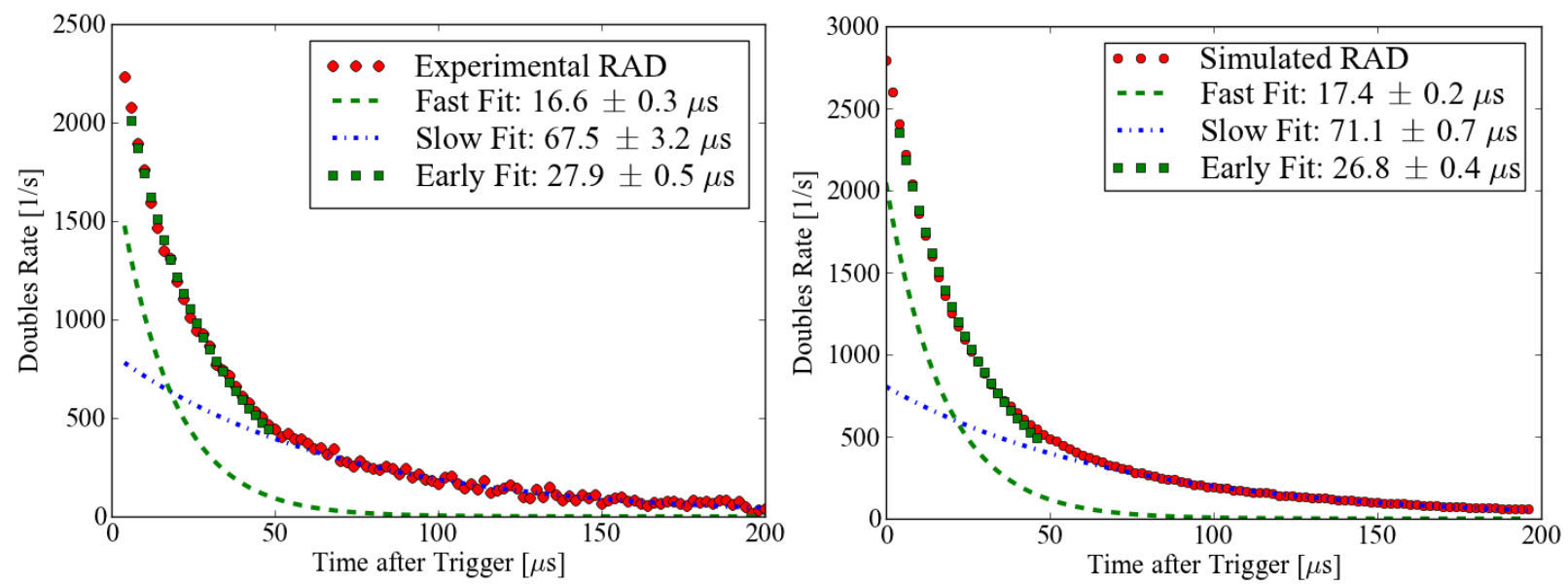

Figure 7-9. Comparison of die-away times of experimental and simulated RADs from $0.98 \%$ enrichment case.

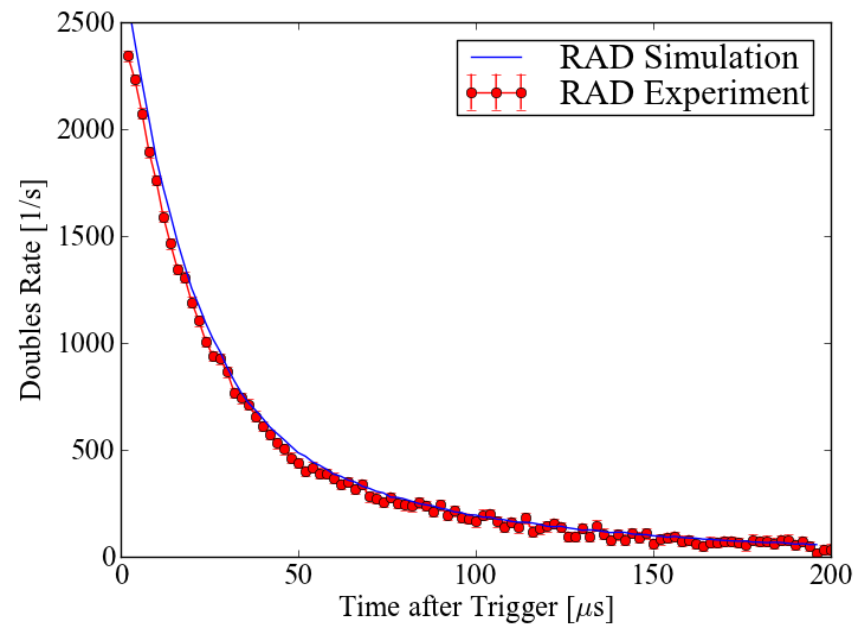

Figure 7-10. Overlain RADs from $0.98 \%$ enrichment case. 

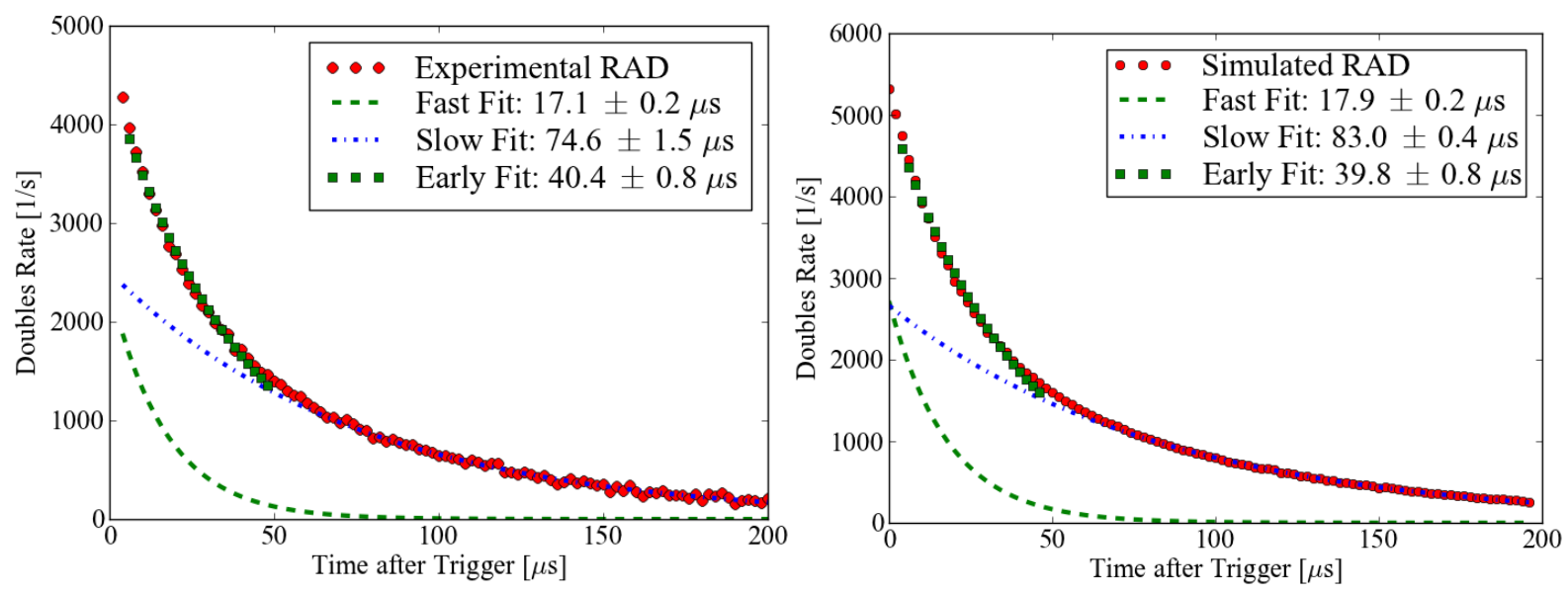

Figure 7-11. Comparison of die-away times of experimental and simulated RADs from 1.97\% enrichment case.

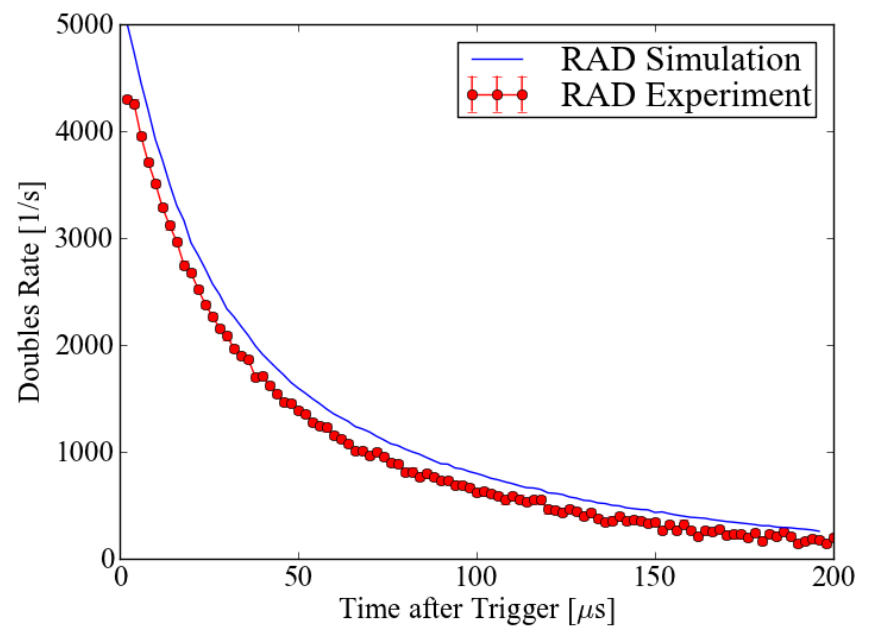

Figure 7-12. Overlain RADs from $1.97 \%$ enrichment case. 

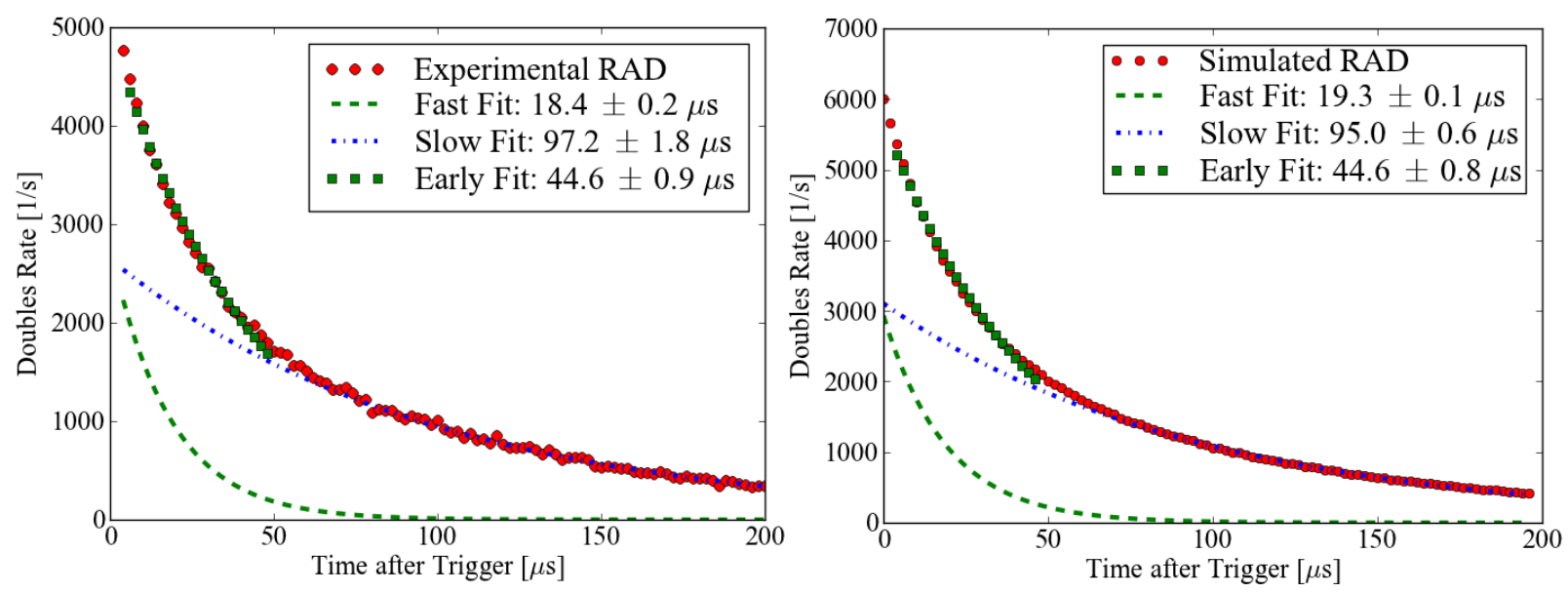

Figure 7-13. Comparison of die-away times of experimental and simulated RADs from 1.67\%, fully asymmetric case.

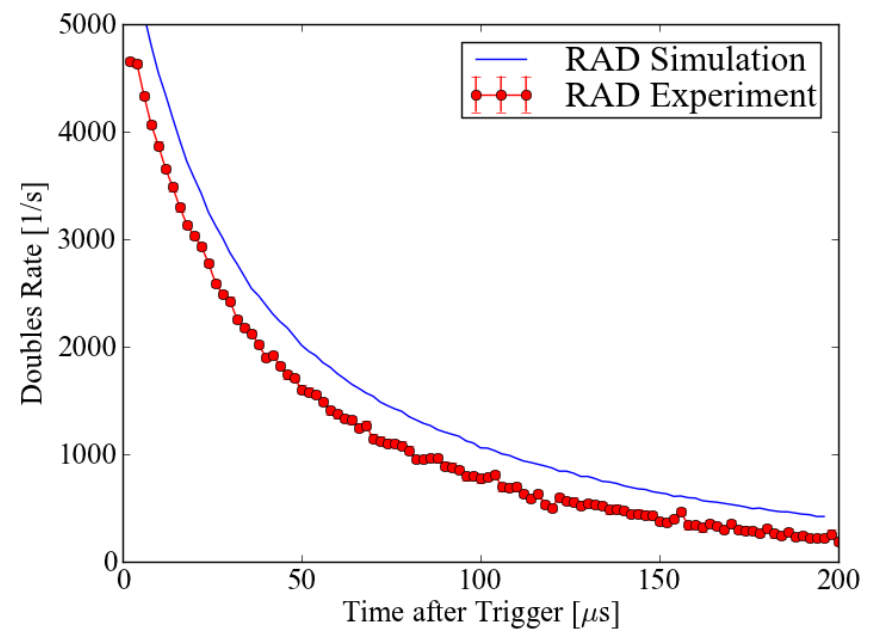

Figure 7-14. Overlain RADs from 1.67\%, fully asymmetric case.

Just as with the singles and doubles rates, the shape and magnitude of the RAD curve matches better in low rate cases. This is reflective of the multiplication disagreement.

Though the magnitude of the fresh fuel measured signal is not comparable to that from spent fuel, the physics properties are quite similar. The presence of the gadolinium rods in certain enrichments and absence in others allows us to compare assemblies with different neutron absorber content as is the case with spent fuel. The varying ${ }^{235} \mathrm{U}$ content allows us to consider different enrichments. Measuring assemblies that are asymmetric parallels the often observed asymmetric burnup of spent 
fuel assemblies. The primary difference (aside from count rate) is the constant spontaneous fission signal. In spent fuel, the spontaneous fission term varies considerably with burnup and cooling time [51] whereas our measurements of fresh fuel have a constant source term from the five stationary ${ }^{252} \mathrm{Cf}$ sources. The result is a singles rate in fresh fuel that changes proportionally to net multiplication as determined from MCNP outputs. This effect is not observed in spent fuel, as shown in Figure 7-15.

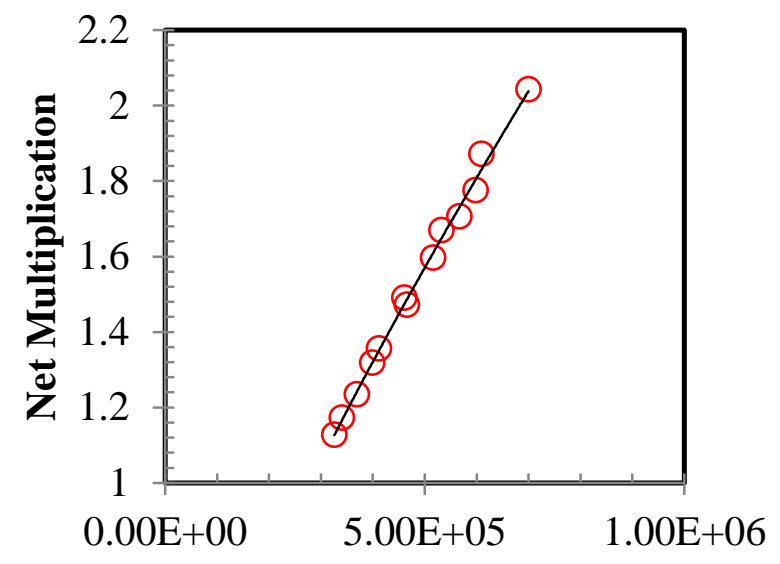

Measured Singles Rate [cps]

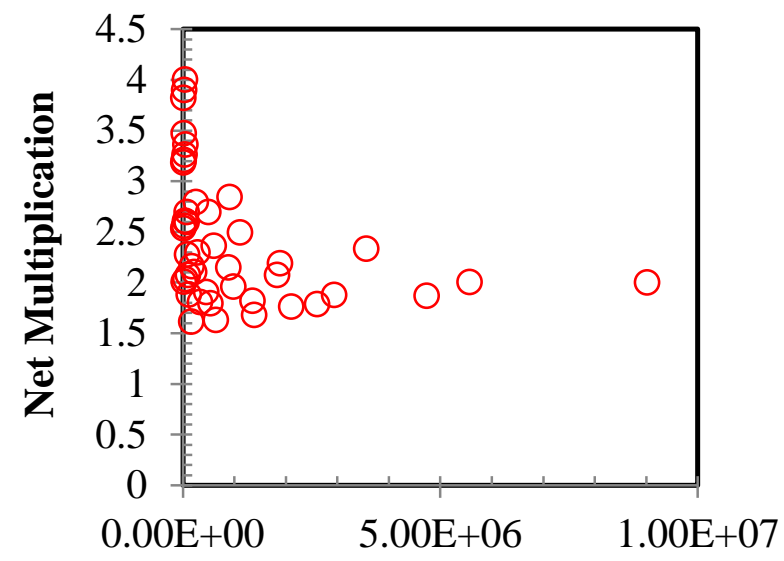

Simulated Singles Rate [cps]

Figure 7-15. Net multiplication as function of singles rate for measured fresh fuel data (left), and simulated spent fuel data (right).

We are interested in applying the early die-away method from [9] and Section 5.1 of this thesis to fresh fuel measurements to ascertain whether or not this relationship exists in experimental results. In fresh fuel, we can recreate aspects of these parameters by observing whether or not the relationship holds for varying neutron absorber content and enrichment. The leakage multiplication can be approximated first by plotting the measured singles rate vs the ${ }^{235} \mathrm{U}$ mass $/ \mathrm{cm}$ of the fuel rod as shown in Figure 7-16 and extending the polynomial fit back to 0 to find the zero-fissile content, nonmultiplying assembly, theoretical singles rate of 267,434 cps. Error bars in Figure 7-16 are smaller than the markers used. Each assembly's measured singles rate can then be divided by the zero-fissile content rate to determine leakage multiplication. The leakage multiplication as a function of experimentally-determined early die-away time is plotted for 16 assemblies with 13 different fuel enrichments, both asymmetric and symmetric loadings, and varying neutron absorber content in Figure 7-17. $1 \sigma$ error bars are shown on the early die-away time. Error bars on the singles rate are smaller than the markers used. 


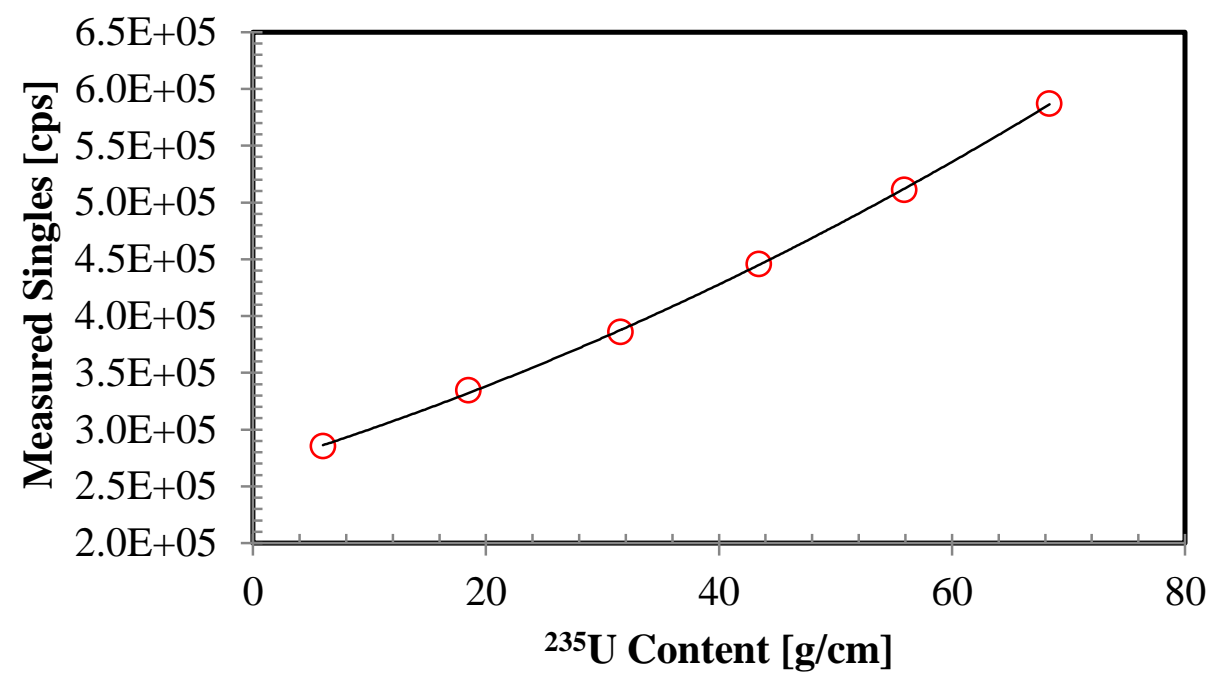

Figure 7-16. Measured singles rate as a function of total ${ }^{235} \mathrm{U}$ content in all fuel rods. Polynomial is extended back to 0 to determine zero-fissile mass singles rate.

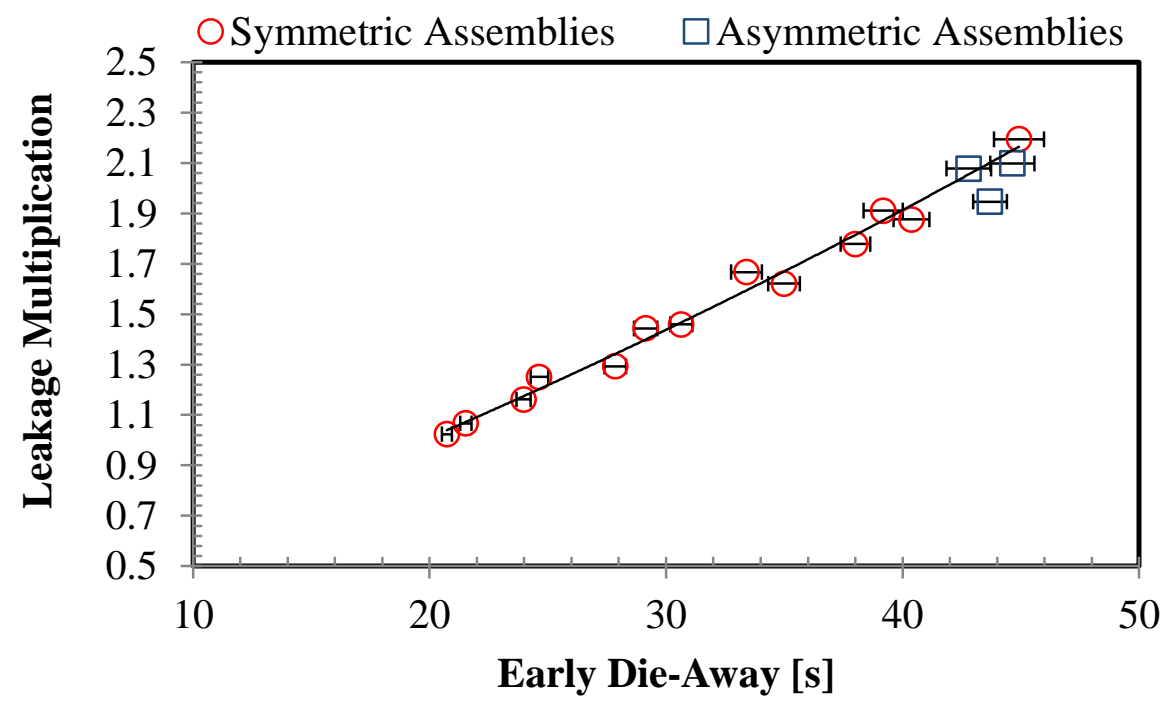

Figure 7-17. Leakage multiplication, which is measured single rate over intercept singles rate, as a function of early die-away time for measured fresh fuel data. Asymmetric assemblies are denoted with square markers.

The same trend that was observed in simulations of spent fuel [9] is apparent in measurements of fresh fuel. Despite varying enrichment, neutron absorber content, and symmetry, early die-away time remains a robust multiplication measurement parameter, predicting multiplication with a RMS error of $2.9 \%$. The fact that the early die-away method is successful with experimental results from varied assemblies in addition to the results from simulated assemblies on which it was developed is a 
promising finding and warrants further investigation and testing of the method in spent fuel measurements.

\subsection{Conclusions}

16 different fresh fuel assemblies were created with a combination of DU, LEU, and Gd rods. 13 of the assemblies were symmetric with different effective enrichments, and 3 assemblies were the same effective enrichment but in different, asymmetric configurations. The assemblies were assayed by the DDSI instrument in two separate measurement campaigns in a water tank at Los Alamos in 2015. Simulations closely reflected the experimental results in cases of low count rate and sources alone with a standard deviation of $0.9 \%$ in the singles and $5.0 \%$ in the doubles. There is a discrepancy in the measured and simulated count rates and die-away times that increased with increasing multiplication/count rate resulting in a standard deviation of $3.8 \%$ in the singles and $14.0 \%$ in the doubles. The merit of the early die-away method developed in simulation space for spent fuel analysis has been validated in measured fresh fuel analysis, lending promise to the method for future use in fuel multiplication determination. Measurements of asymmetric fresh fuel assemblies showed the same trends as symmetric assemblies, indicating that asymmetrically burned spent fuel assembly measurements will not result in inaccuracies in the analysis methods. 


\section{Chapter 8. RoSSI-AlPHa DiSTRIBUTIONS WITH ORgANIC LIQUID SCINTILLATORS}

The DDSI technique as described in this thesis utilizes the thermal neutron self-interrogation of fissile isotopes to determine sample multiplication. In fuel measurements, neutrons may thermalize in water between fuel rods enabling interrogation evenly across the assembly. Alternatively, when measuring a thick sample with only exterior moderating material, measured thermal fission multiplication comes only from the outer edge of the item $(\sim 2-3 \mathrm{~mm})$, because thermal neutrons from the moderating material cannot travel deep into the item before causing fission. Fast fission, however, is induced throughout the volume of the item because of the greater penetrability of fast neutrons. Fast multiplication, or the multiplication as a result of fast fissions, occurs on too short of a time scale that is significantly shorter than the characteristic die-away time of any ${ }^{3} \mathrm{He}$ detectorbased instrument. The die-away times of these instruments are considerably longer than the time of between subsequent fast fissions, making the dynamic evolution of the neutron population due to fast fission impossible to observe. Organic liquid scintillators, however, have a time constant that is substantially shorter than that of ${ }^{3} \mathrm{He}$-based systems and therefore are proposed to be used to study the dynamic evolution of fast multiplication in a thick, multiplying item with an exterior reflector. Such approach could be useful in arms control and Nuclear Nonproliferation Treaty verification applications where fast multiplication could assist in characterizing the item of interest.

\subsection{Description of Experiment}

To examine the ability of organic liquid scintillators to be used for fast multiplication observation, data from a measurement campaign conducted by Scott Kiff in $2012\left[{ }^{97}\right]$ were reanalyzed using DDSI algorithms developed in this thesis, with RADs as the center point. In these experiments, the 
beryllium reflected plutonium (BeRP) ball was measured with various reflectors. The BeRP ball is a sphere of $99.58 \%$ plutonium with the rest of the mass being primarily iron, chromium, nickel and manganese. The mean radius is $3.7938 \mathrm{~cm}$ and the mass is $4483.884 \mathrm{~g}$. Four $3 \times 3$ " EJ-309 organic liquid scintillators were arranged in an array $15 \mathrm{~cm}$ away from the edge of the BeRP ball as shown in Figure 8-1 for the experiments. Five measurements were performed: bare, 0.5 " W reflector, 1 " W reflector, 1" HDPE reflector, and 3" HDPE reflector. The collection times and neutron detection thresholds in the liquid scintillators varied from case to case, although the exact information has unfortunately not been preserved. The information available based on laboratory notebooks is listed in Table 8-I.

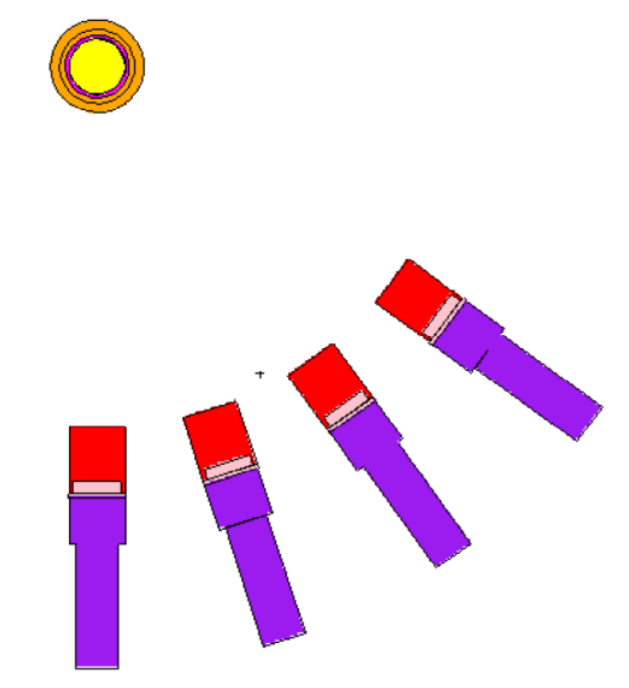

Figure 8-1. BeRP ball measurement setup. Image from [98].

Table 8-I. Experimental parameters for different BeRP setups.

\begin{tabular}{ccc}
\hline Case & Time (min) & Threshold \\
\hline Bare & 92 & $0.158 \mathrm{~V}$ \\
0.5” W & 60 & $0.069 \mathrm{~V} *$ \\
1" W & 150 & $0.069 \mathrm{~V}$ \\
1" HDPE & 60 & $0.122 \mathrm{~V} *$ \\
3" HDPE & 42 & $0.122 \mathrm{~V}$ \\
\hline
\end{tabular}


Table 8-I values with an asterisk (*) are assumed values based on the lab notebook. The explicit values are not given for those setups, rather, the 0.5 " W and 1 " HDPE threshold values are logged as "lowered" and "raised", respectively. It is assumed that they are the same for both thicknesses of the same reflector material. The exact magnitude of these values is not essential to this analysis and therefore the assumption is not detrimental to the integrity of the results.

\subsection{Results}

The base case, or reference case, is the measurement of the BeRP ball with no reflector material around. This setup should have the lowest multiplication of the various setups as it has no material to reflect neutrons back into the item. The RAD from the base measurement with accidentals subtracted is given in Figure 8-2. Because of the lack of reflector, the RAD is fit with a single exponential as would be done with a ${ }^{3} \mathrm{He}$-based instrument RAD, however the timescale is two orders of magnitude lower. Bin widths of $20 \mathrm{~ns}$ are used in order to produce adequate statistics while still maintaining the ability to observe die-away behavior of the curve. $1 \sigma$ statistical uncertainty is given on the exponential fit, which is performed excluding the time domain of 0-40 ns in which the signal build up occurs.

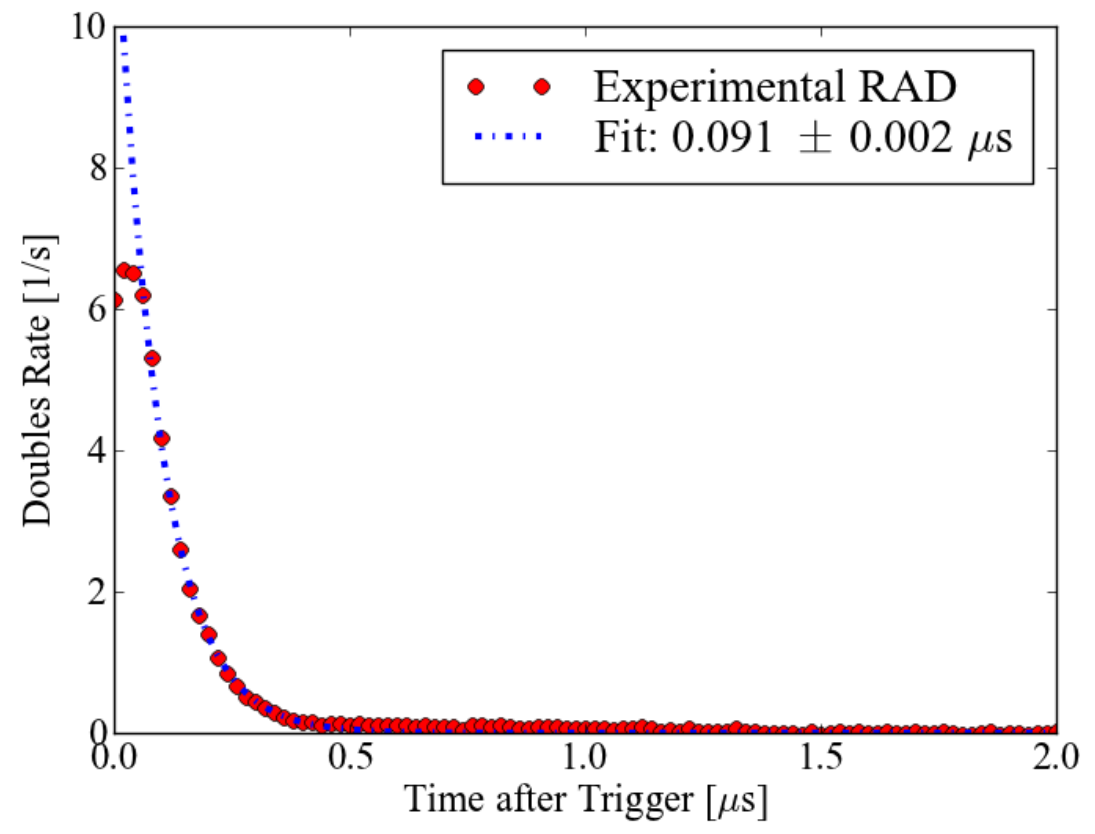

Figure 8-2. RAD from bare BeRP ball setup with exponential fit shown. 
The observed die-away time of $91 \mathrm{~ns}$ is 2-3 orders of magnitude faster than that observed with ${ }^{3} \mathrm{He}$ detectors. A neutron signal rise-time is observed from the signal build-up within the BeRP ball.

The RADs from the different reflector setups can be normalized for easier comparison. The RADs from the measurements of the BeRP ball with reflectors are normalized to the bare BeRP case at 20 ns to compare the RAD shapes in Figure-8-3.

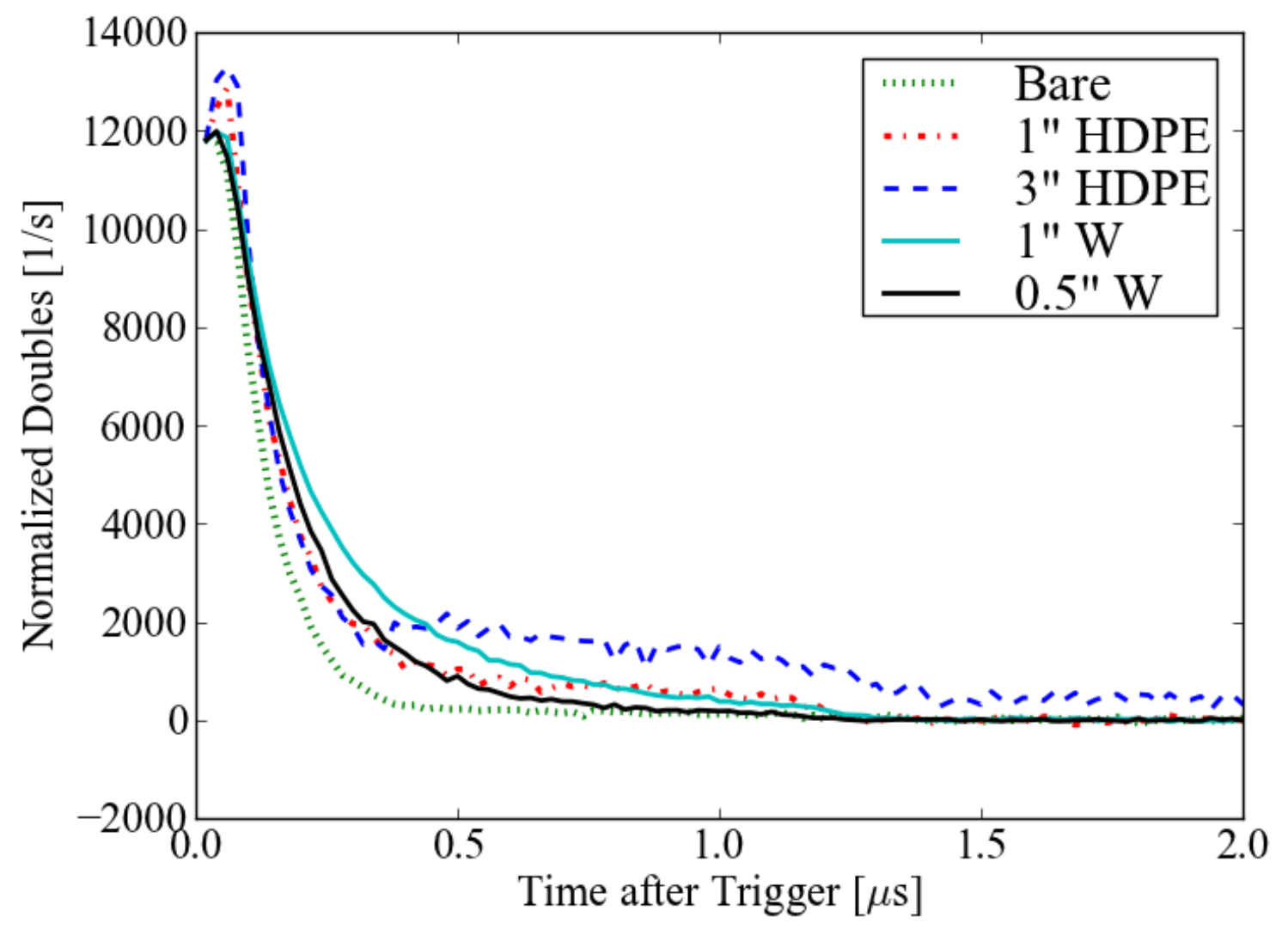

Figure-8-3. Normalized RADs for BeRP measurement setups.

Comparison of the normalized RADs reveals interesting characteristics of the individual BeRP ball measurements. The bare setup has nearly no signal build-up and dies away the fastest, which is commensurate with that setup having the lowest multiplication of any kind because of the lack of reflector material. Also, the tungsten reflected BeRP ball displays very little signal build-up in the early time domain because tungsten does not reflect neutrons back into the item as readily as a lower $\mathrm{Z}$ material would. The tungsten cases do have slower die-away in the early time domain of 0-500 ns, however. This is likely the result of $(n, 2 n)$ reactions taking place in 
the tungsten. The HDPE reflectors behave differently because of the low $\mathrm{Z}$ of HDPE. There is, however, a prominent signal build-up region from fast multiplication within the BeRP ball in the time domain of 0-100 ns. The HDPE RADs drop very quickly in the early time domain likely because there are no $(n, 2 n)$ reactions in these setups, however they display a second, longer die-away time around 400 ns where additional neutrons, likely from fast fission, are populating the RADs. Neutrons that thermalize in the HDPE will only penetrate a shallow distance into the BeRP ball and induce thermal fissions. Moreover, due to the time associated with thermal diffusion, the timescale of this process is considerably longer than what is being considered in Figure-8-3. The fast multiplication, however, will occur when neutrons are reflected directly back into the item without losing much energy.

The RADs can be broken down into fast and slow components as in typical DDSI type of analysis (although much shorter time scale) for die-away time analysis. The fitted RADs for each setup are given in Figure 8-4 through Figure 8-7. $1 \sigma$ statistical uncertainties are given on the die-away times. 


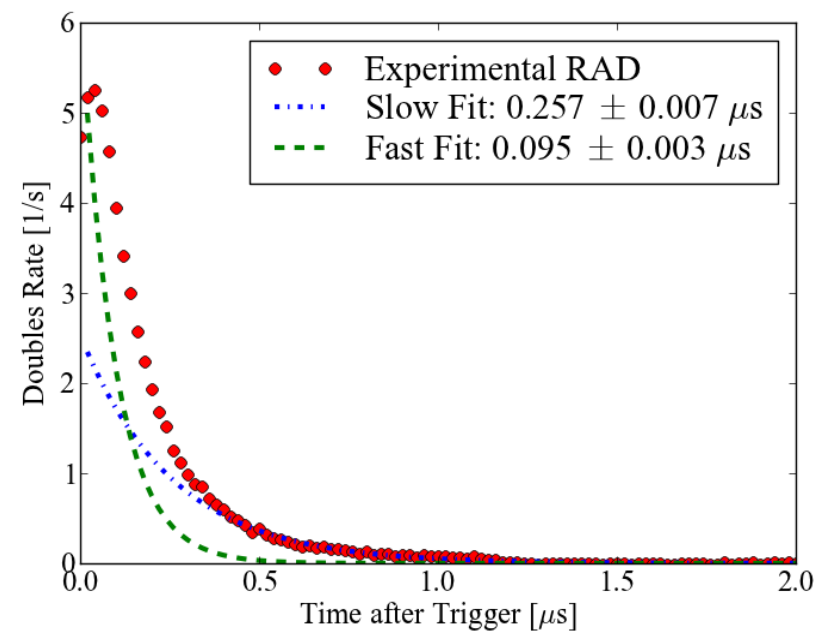

Figure 8-4. RAD from measurement with 0.5 " W reflector, with fast and slow curves shown.

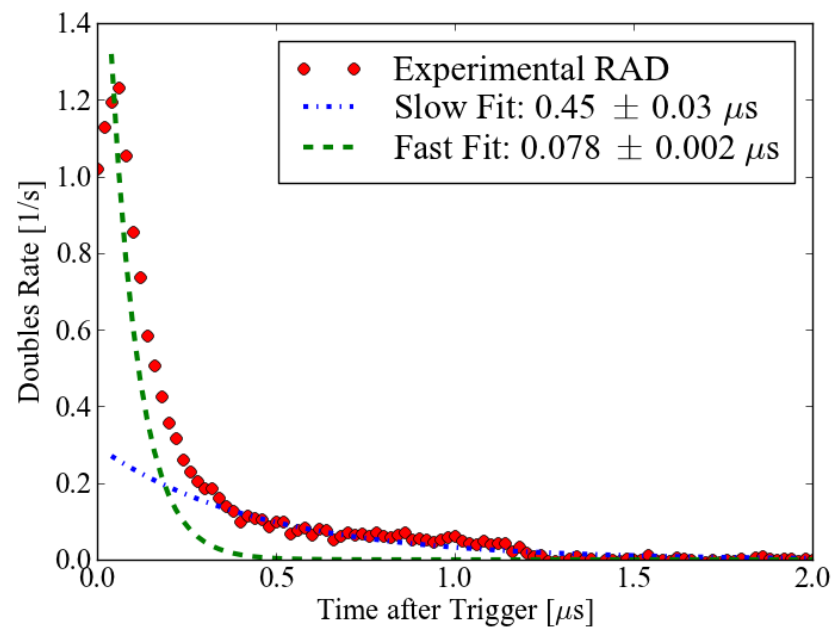

Figure 8-6. RAD from measurement with 1" HDPE reflector, with fast, slow, and early curves shown.

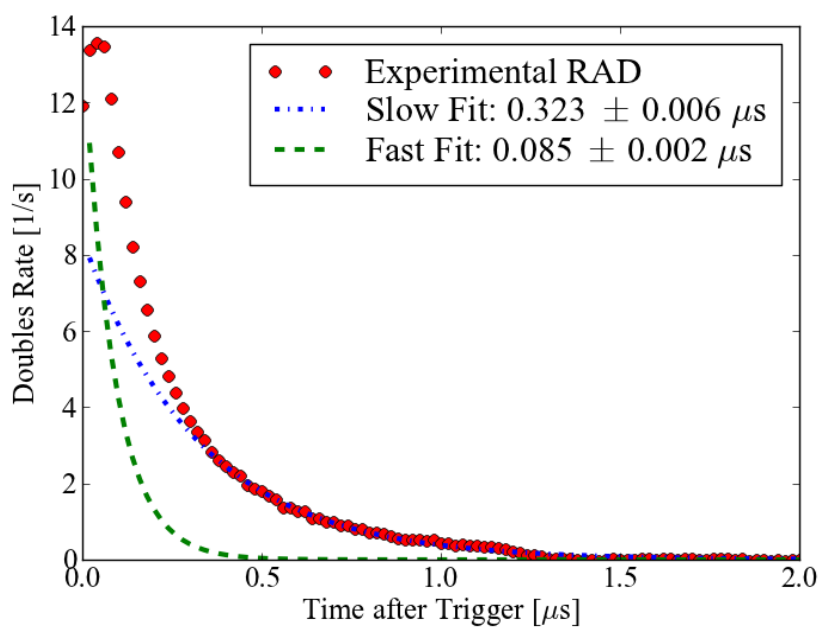

Figure 8-5. RAD from measurement with 1" W reflector, with fast and slow curves shown.

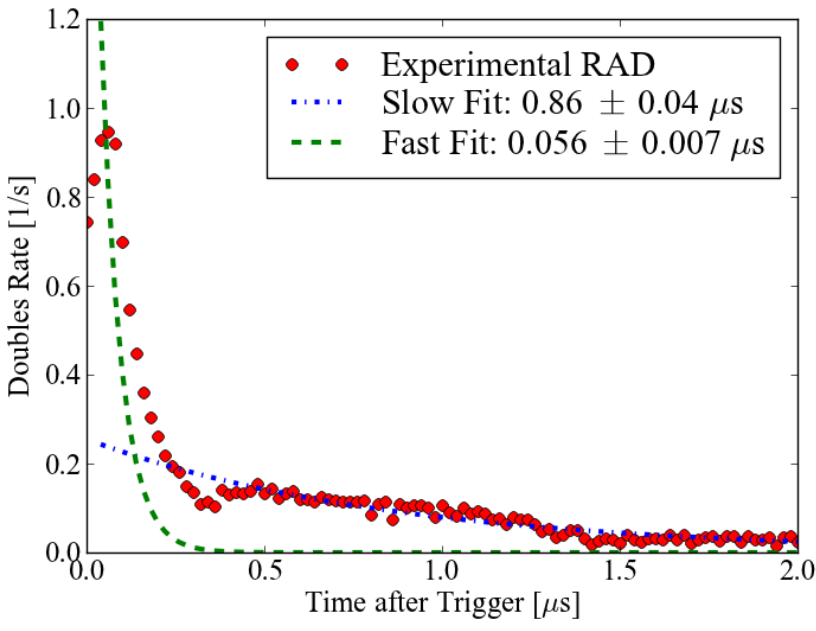

Figure 8-7. RAD from measurement with 3" HDPE reflector, with fast, slow, and early curves shown.

The uniquely fast die-away of liquid scintillator RADs allows for observation of the result of different effects on the RAD about which we may at this point only speculate. The fast component of the tungsten-reflected cases is slightly longer with respect to the bare case likely because of the $(\mathrm{n}, 2 \mathrm{n})$ neutrons. The slow component of the HDPE-reflected cases is longer likely because of the increased fast fission multiplication resulting from the back scatter of neutrons interactions in the 
reflector, and subsequent thermal induced fission which creates neutrons that can cause additional fast fissions The RADs also have unique features in their shapes that cannot be seen in data from ${ }^{3} \mathrm{He}$ based instruments such as the sudden drop that occurs slightly after $1 \mu$ s in each case, and the slight drop in counts that occurs at approximately $400 \mathrm{~ns}$ in the 3 in HDPE case. Additional research could be done utilizing the uniquely fast timing of liquid scintillator RADs to learn more about fast multiplication in items and how it is affected by moderators, as well as possibly incorporating neutron energy to determine fast multiplication.

\subsection{Conclusions}

Differential die-away self-interrogation in the traditional sense cannot be used to determine multiplication in thick items such as the BeRP ball because of the shallow penetration of thermal interrogating neutrons. However, fast neutrons can travel throughout such an item and induce fission, and through use of fast detectors such as liquid scintillators, the properties of fast multiplication can likely be observed. The fast RADs were presented along with preliminary analysis of their die-away times. Future work could be done with this type of analysis to learn more about fast multiplication, and incorporating neutron energy could further enable the multiplication determination of thick items with exterior moderator for arms control or Nuclear Nonproliferation Treaty verification applications. 


\section{Chapter 9. Conclusions}

\subsection{Contributions of this Thesis}

The DDSI instrument has been investigated as a new nondestructive assay technique for characterizing spent nuclear fuel. Its capabilities have been analyzed quantitatively and qualitatively. It was determined that Rossi-alpha distributions created with list mode data from the DDSI instrument can be used to quantify elemental Pu mass of a spent fuel assembly with a RMS error of $2.1 \%$ in simulation space, as demonstrated in Section 5.2. The technique developed could also theoretically be applied to list-mode data collected with another instrument. Using a reference assembly approach, RADs produced with the DDSI instrument can also be used to identify all assemblies investigated in this work, each of which had $18 \%$ of pins diverted and replaced with dummy materials. This is a $32 \%$ improvement upon the going standard in the Safeguards community of $50 \%$ diversion detection. Through this work the early die-away method was developed as well. This method can be used to determine leakage multiplication with a RMS error of $0.8 \%$ in simulation space, as demonstrated in Section 5.1, and a RMS error of $2.9 \%$ in experiments, as demonstrated in Section 7.3. In certain applications it will be a considerable improvement upon other multiplication determination methods because it is independent of the $(\alpha, n)$ source term and neutron absorber content. The instrument was used to conduct the first fresh fuel measurements with the DDSI method and results

\subsection{Proposed Future Work}

Additional work needs to be done to fully demonstrate the viability of the DDSI instrument for use in characterization of spent nuclear fuel. The instrument has been tested with fresh fuel and high count rate scenarios; however actual placement in a spent fuel pool and measurement of a varied set of spent fuel assemblies will allow for validation of the early die-away method and other methods 
presented in this thesis. The assemblies measured need to vary widely in initial enrichment, burnup, cooling time, and other fuel parameters if possible in order to verify that the techniques can be applied to heterogeneous fuel sets as would be encountered if the instrument were used for future verification purposes at a facility. It is recommended that partial defect, or pin removal, tests be performed with spent fuel to test the reference assembly approach presented here as well as the plutonium diversion approach.

Additional work could be done with initial enrichment, burnup, and cooling time determination by incorporating gamma-ray measurement data. The instrument has the capability to measure total gamma and while work has been done on passive gamma NDA in other contexts, combining that signal with the neutron RAD could be informative and could expand the IE, BU, CT determination capabilities.

It is also proposed that the PWR analysis methods be expanded to BWR assemblies in simulation space. Once methods are adapted for the different assemblies, fresh fuel BWR measurements could be performed as well as spent fuel measurements, in time. This would be a useful investigation for the purposes of the IAEA and future inspection scenarios to determine whether DDSI is a viable BWR measurement technique.

Spectral analysis could be used to delve deeper into the information stored in Rossi-alpha distributions. This technique uses Fourier transforms to deconstruct decaying curves. It could provide precise decay times and could possibly be used to obtain more information than the double exponential fit from RADs.

Finally, application of the Rossi-alpha distribution analysis to data taken with organic liquid scintillators should be expanded and further tested. Though preliminary results in this thesis demonstrate that fast multiplication may be reflected in BeRP ball measurements with the organic liquid scintillators, it would be useful to expand the analysis to consider neutron energy and different reflectors, as well as to conduct thorough simulations of the setups. Rossi-alpha distributions may be useful tools in arms control applications and with further testing, the analysis could be applied to more varied measurement scenarios and help in expanding understanding of fast multiplication. 


\subsection{Summary and Conclusions}

Nondestructive assay of spent fuel will always be a key component of international safeguards due to the need for on-site, rapid measurements. Fuel to be lowered into a repository or reprocessed needs to be well-characterized, and destructive assay is not always an option. The Differential Die-Away Self-Interrogation instrument was developed with the intent of creating a new, nondestructive method capable of accurately characterizing spent nuclear fuel. This instrument was the first neutron coincidence counting-based technique capable of measuring spent fuel. The design concept was optimized through a series of high fidelity simulations over several years. During that time, individual smaller components were built and tested. Detector pods were benchmarked and tested for temperature stability and gamma sensitivity. The data acquisition system was tested extensively and its performance was improved through a series of modifications. Analysis scripts were also written during this time to be applied to both simulated and experimental data.

Spent fuel was simulated and analyzed in detail to develop an understanding of ingrowth of transuranics including fissile isotopes, neutron absorbers, and spontaneous fission isotopes. The interaction of these isotopes in spent fuel was investigated and the physics of Rossi-alpha distributions was explored in order to develop and test novel methods of fuel characterization.

The early die-away method was developed, which is an improved, robust way of measuring assembly multiplication independent of the $(\alpha, n)$ source term, operating parameters, or fissile/neutron absorber content. This method was determined to be capable of measuring multiplication of assemblies in real-life measurements of fresh fuel. It was also extended beyond fuel assemblies and used in the arms control context for measurements of weapons-grade plutonium. The next step for this method is to measure spent fuel assemblies and test the performance.

In addition to the early die-away method, a technique for measuring total $\mathrm{Pu}$ with DDSI was presented and tested on a variety of simulated spent fuel assemblies. Two alternative approaches to missing pin detection were also presented, as well as IE and BU determination algorithms. These can all again be tested with spent fuel measurements.

Measurements conducted with the DDSI instrument were used to characterize the instrument's efficiency, die-away time, and deadtime. The efficiency of a ${ }^{252} \mathrm{Cf}$ point source in the center of the instrument was $12.0 \% \pm 0.1 \%$. The die-away time was $18.1 \pm 0.1 \mu$ s and the deadtime was $23 \mathrm{~ns}$. 
They were also used to expose the electronics to extremely high count rates in order to evaluate the limits of the instrument's detection capabilities under those challenging conditions. The instrument and data acquisition system performed well under the high count rate conditions and it can therefore safely be assumed that the high neutron and gamma fluxes from spent fuel will not inhibit the instrument's ability to perform characterization measurements.

Fresh fuel assemblies in 16 different configurations were measured at Los Alamos National Laboratory with the DDSI instrument. The early die-away method was applied to the results of the fresh fuel measurements and it was found that the early die-away time determined experimentally trended with the net multiplication. The assemblies used had different enrichments and neutron absorber content, and were both symmetric and asymmetric. The robustness of the early die-away method was demonstrated by showing its successful application to this widely varied data set.

Rossi-alpha distributions produced from data collected with organic liquid scintillators were considered for their unique ability to reflect fast multiplication in an assayed item. The distributions demonstrate trends congruent with what would be expected from fast detector measurements, and further work could enable fast multiplication determination from such experiments for arms control and treaty verification applications.

The Differential Die-Away Self-Interrogation instrument is a promising new nondestructive assay technique that was shown to be capable of analyzing spent fuel. The instrument fills gaps in NDA capabilities such as sensitive pin diversion detection and robust multiplication determination without a priori knowledge of the $(\alpha, n)$ source term or constant geometry. The development of the new, improved characterization and verification methods with the DDSI instrument makes it a viable technique for implementation in a facility to meet material control and safeguards needs. 


\section{APPENDICES}

\section{Appendix A. Spent Fuel Measurement with Liquid Scintillator Feasibility Study}

Spent fuel assay has been a focus of research internationally for several years and continues to be of significant importance to international safeguards [99]. The timing characteristics of neutrons emitted from spent fuel contain valuable information about the assembly being measured and therefore this information is frequently utilized in new Nondestructive assay (NDA) methods [100]. The traditionally employed He-3 detectors have relatively slow timing characteristics and thus are not ideal for coincidence measurements [41]. In contrast, the fast timing of EJ-309 organic liquid scintillators make them attractive alternatives for spent fuel measurements.

The challenge posed to these detectors is that they detect both gamma-rays and neutrons, and therefore may not be able to detect the small neutron fluence in the presence of the dominating gamma-ray fluence emitted from spent fuel. To be utilized in this context, it must be shown that the particle misclassification rate of the measurement system is low enough not to significantly distort the neutron signal measured [101]. Work has been performed in the past to evaluate EJ-309 neutron detection in a high gamma-ray background with a threshold of approximately 200 keVee [102] and $500 \mathrm{keVee}$ [103]. The research presented in this paper alternatively applies a low threshold of 50 keVee to evaluate pulse-shape discrimination (PSD) in this low-pulse-height region. We apply a systematic post-measurement PSD algorithm to separate the particles and the effectiveness of the algorithm is quantified by comparing neutron pulse-height distributions.

\section{Experiment}

\subsection{Setup}

The experiment was conducted with a 3x3" EJ-309 organic liquid scintillator coupled with an ETL 9821 photo-multiplier tube (PMT). Pulses were processed with a CAEN V1720 eight-channel, 12- 
bit, 250-MHz digitizer. Calibrations were performed before each measurement with a $1 \mu \mathrm{Ci}{ }^{137} \mathrm{Cs}$ source affixed directly to the detector face. A negative high voltage ranging from -1760V to -1770V was applied to the detector and was varied with each calibration to keep location of the ${ }^{137} \mathrm{Cs}$ Compton edge constant with each measurement. Waveforms were collected with 120 samples per pulse.

Two sources were used in this set of measurements: a $7 \mu \mathrm{Ci}{ }^{252} \mathrm{Cf}$ source and a $95 \mu \mathrm{Ci}{ }^{137} \mathrm{Cs}$ source. The ${ }^{252} \mathrm{Cf}$ source emits approximately 30,000 neutrons and 120,000 gamma-rays per second, and the ${ }^{137} \mathrm{Cs}$ source emits approximately three million gamma-rays per second. In order to vary the gammato-neutron $(\gamma / \mathrm{n})$ ratio with the two sources, their distances relative to the detector were varied. The case with the lowest $\gamma / \mathrm{n}$ ratio is the ${ }^{252} \mathrm{Cf}$ source placed $10 \mathrm{~cm}$ from the detector, which will henceforth be referred to as the ${ }^{252} \mathrm{Cf}$ alone case. The other distances are shown in Fig. A-1.

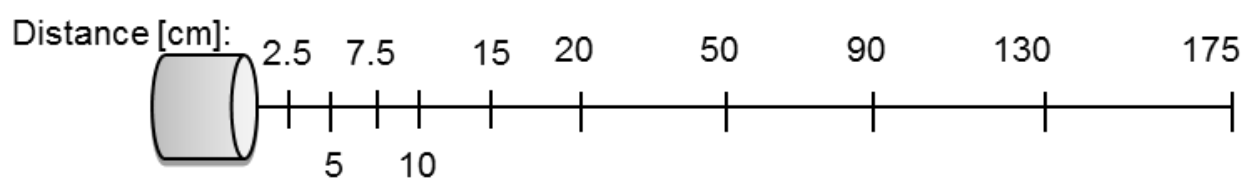

Figure A-1. Visual representation of the source distances from the detector face. ${ }^{252} \mathrm{Cf}$ is stationary at a distance of $10 \mathrm{~cm}$ and ${ }^{137} \mathrm{Cs}$ is placed at varying distances from the detector, indicated by the tick marks.

For all measurements, the ${ }^{252} \mathrm{Cf}$ was positioned $10 \mathrm{~cm}$ from the detector face, resulting in a total count rate of approximately 900 counts per second. The ${ }^{137} \mathrm{Cs}$ was moved from the minimum distance of $2.5 \mathrm{~cm}$ to the maximum of $175 \mathrm{~cm}$ to allow for observation of a wide range of theoretical $\gamma / \mathrm{n}$ ratios from approximately $770 \pm 25$ to $5.6 \pm 0.2$. The ${ }^{252} \mathrm{Cf}$ source was placed on a porous foam support raised to $1.5 \mathrm{~cm}$ below the center plane of the detector to a height of $12 \mathrm{~cm}$ from the table. The ${ }^{137} \mathrm{Cs}$ source was placed directly above the ${ }^{252} \mathrm{Cf}$ source, so with a thickness of $0.5 \mathrm{~cm}$, the top of the ${ }^{137} \mathrm{Cs}$ was $12.5 \mathrm{~cm}$ above the table and $1 \mathrm{~cm}$ below the center plane of the detector. A photograph of the experimental setup for the ${ }^{137} \mathrm{Cs}$ source placed at $5 \mathrm{~cm}$ from the detector face and the ${ }^{252} \mathrm{Cf}$ source placed at $10 \mathrm{~cm}$ from the detector face is shown in Fig. A-2. 

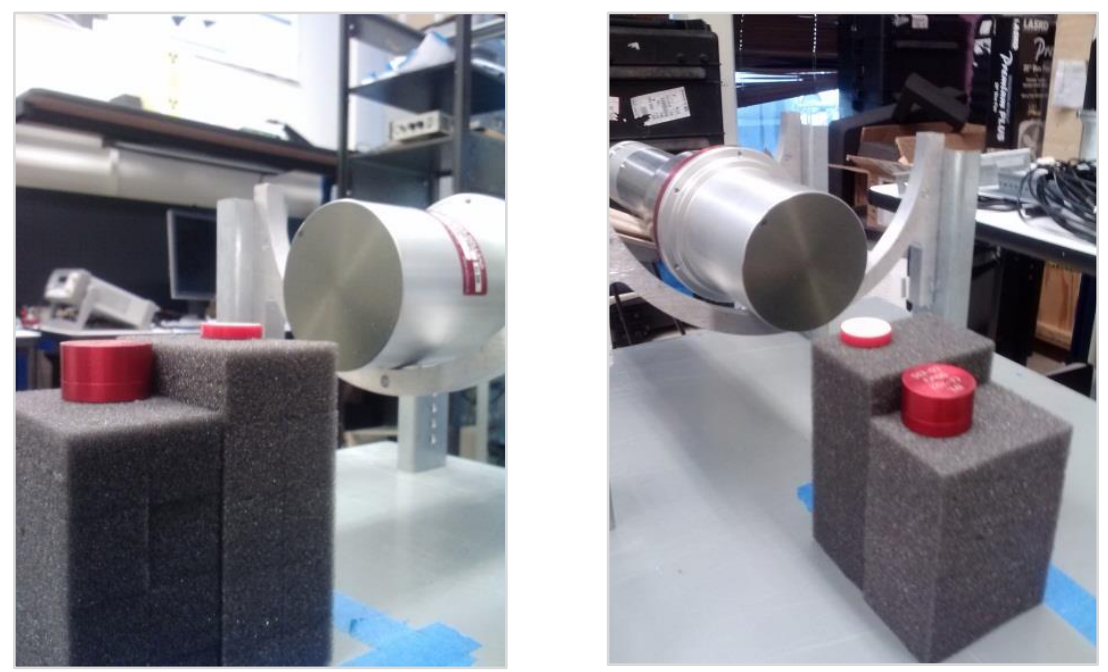

Figure A-2. Experimental setup for the ${ }^{137} \mathrm{Cs}$ source placed at $5 \mathrm{~cm}$ from the detector face and ${ }^{252} \mathrm{Cf}$ source at $10 \mathrm{~cm}$ from the detector face.

The measurement threshold was set to $20 \mathrm{keVee}$, corresponding to approximately $27 \mathrm{keV}$ gammaray energy and $300 \mathrm{keV}$ neutron energy deposited. This low threshold was chosen to allow for variation in threshold in data post-processing.

\subsection{Count Rates}

Combined count rates were recorded for all cases and are plotted in Fig. A-3. The ${ }^{137}$ Cs count rate was recorded alone at each distance first, then the ${ }^{252} \mathrm{Cf}$ source was added and the new count rate was recorded. The ${ }^{137} \mathrm{Cs}$ source is considerably stronger than the ${ }^{252} \mathrm{Cf}$ source and it dominates until the ${ }^{137} \mathrm{Cs}$ is at a distance of $50 \mathrm{~cm}$. 


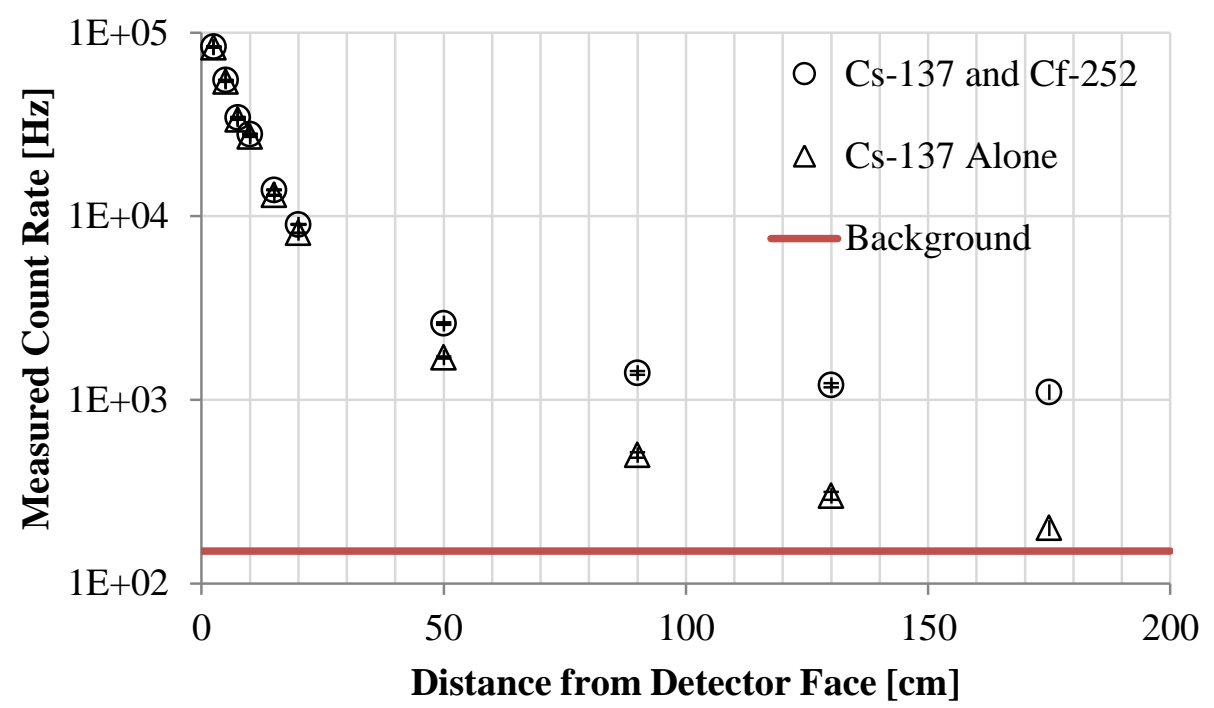

Figure A-3. Count rates as a function of ${ }^{137} \mathrm{Cs}$ distance from detector face, without background subtraction. The count rate of the ${ }^{137} \mathrm{Cs}$ source at the distance of $175 \mathrm{~cm}$ is just above the background gamma count rate.

The triangles in Fig. 3 are the count rates with no ${ }^{252} \mathrm{Cf}$ source present to demonstrate that at the furthest distance of $175 \mathrm{~cm}$, the count rate of the ${ }^{137} \mathrm{Cs}$ alone is just above background. The highest count rate was recorded when the ${ }^{137} \mathrm{Cs}$ was placed at a distance of $2.5 \mathrm{~cm}$ from the front face of the detector, and was close to 100,000 counts per second.

\subsection{Pileup Rejection}

As the ${ }^{137} \mathrm{Cs}$ source is positioned closer to the detector, the number of double pulses will increase. A pileup rejection algorithm was applied to eliminate these pulses which, if included with the data, would produce incorrect results for the neutron pulse-height distributions. Clipped pulses were identified as those that had clipped peaks. The pulse clipping occurs as the result of the finite dynamic range of the digitizer $(\sim 2 \mathrm{~V})$. All clipped pulses were removed from the measured data. Double pulses were identified as those having a second pulse with a leading edge that increased by at least 5\% of the height of the first pulse in one digitizer step (two adjacent digitizer samples are $4 \mathrm{~ns}$ apart). The second pulse must arrive within approximately $400 \mathrm{~ns}$ of the primary pulse to be eliminated as a double, however it must arrive later than $16 \mathrm{~ns}$ which is the deadtime of these detectors. Examples of rejected pulses are shown in Fig. A-4. In this experiment, the conservative $5 \%$ threshold is used in order to prevent "false neutrons" that are created when a second pulse arrives within a small time window of the primary gamma, resulting in a misleadingly large tail integral. 


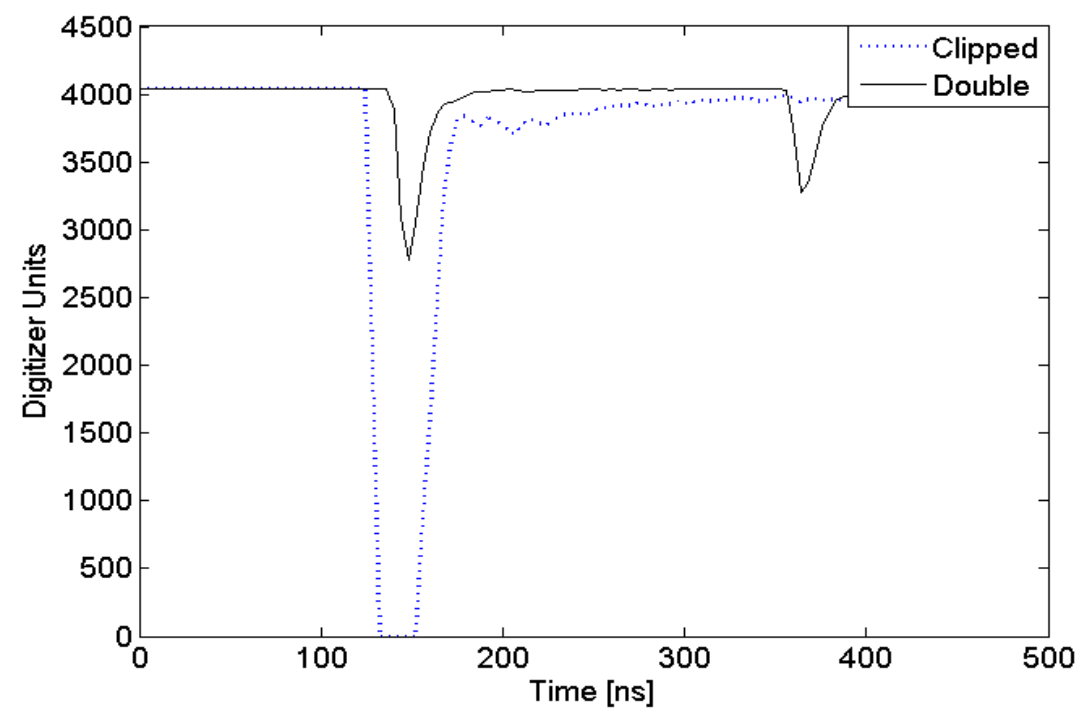

Figure A-4. Examples of clipped and double pulses.

\section{Pulse-Shape Discrimination Method}

The PSD method employed was digital charge-integration PSD [104]. In this method, each collected waveform is integrated in two ways: the tail, or region of the pulse from a set time after the peak to the end, and the total pulse. The time after the peak to begin tail integration was chosen to be $20 \mathrm{~ns}$ based on previous studies [105] but the optimal choice for this value is not fixed and is in fact varied by several experimental parameters such as cable length, detector size, and sample rate $\left[{ }^{106}\right]$. Because the fraction of light that appears in the slow component of the pulse is larger for neutrons than for gamma-rays, the tails of the neutron waveforms will be larger. This property allows for particle discrimination. A systematic algorithm utilized the pulse shape discrimination results to find an optimal separation line. The case of ${ }^{137} \mathrm{Cs}$ at $50 \mathrm{~cm}$ is used to determine the separation line because at this distance, there is sufficient separation between neutron and gamma-ray distributions to determine a clear line of distinction; however, the count rate is still high enough to provide good statistics. A line determined using any distance measurement should be able to be applied to all other distances because the distributions should not shift or change if measurement parameters are kept constant. The PSD plot from the $50 \mathrm{~cm}$ case containing tail integrals vs. total integrals was sliced with a constant slope perpendicular to the gamma-ray region to isolate at least 2000 pulses per slice. Each time a slice is created, the points that fall within that slice are isolated and split into subsections perpendicular to the slice, as shown in Fig. A-5. 

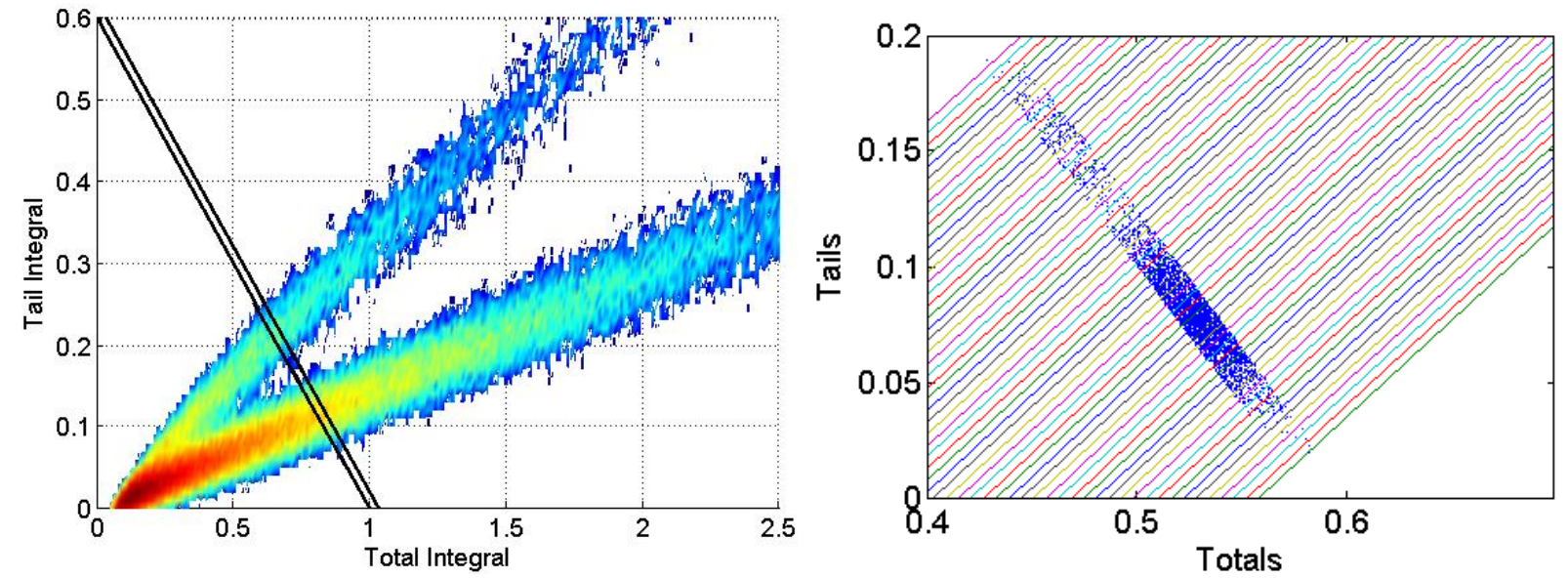

Figure A-5. Example of taking a light output "slice" from the 2-D plot, and slicing again perpendicular.

Now that there are uniform subsections of the already sliced data, we can find the minima in the bins where there is optimal gamma-neutron separation. This is done simply by counting the number of pulses that fall within each perpendicular slice and finding the minimum. If multiple minima occur in a slice, the center minimum index is used. When this process has been repeated for several slices, the result is optimal separation points chosen systematically from slices that logically increase in size as points get less dense. This approach is shown in Fig. A-6.
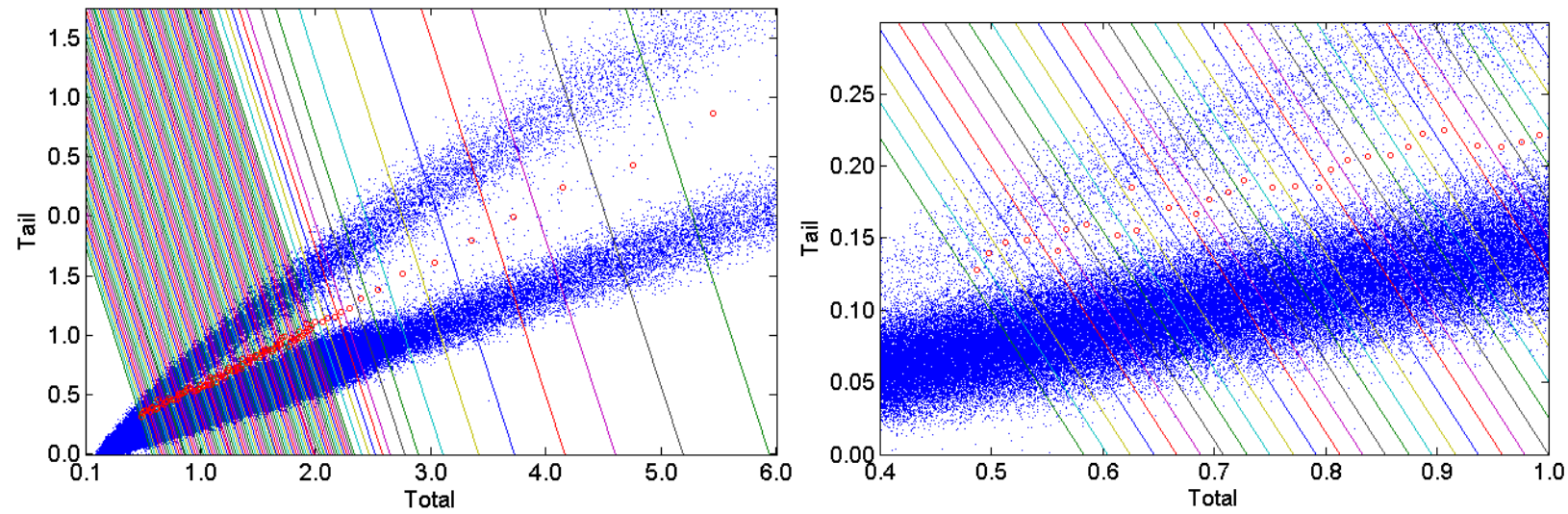

Figure A-6. Entire PSD plot sliced with optimal separation points found. Zoomed version on the right.

A check is employed to ensure that within a single measurement, there is no significant shift within the data. Two portions of the same measurement taken 2 hours apart are compared to validate this assertion, and it was clear that no significant shift took place. This same test was performed for 
random segments of other measurements. No discernible difference is found between any of the measurements, so the assumption that the distributions are not shifting is valid.

The optimized line from the $50 \mathrm{~cm}$ case will be applied to all measurement distances. The discrimination curve, as it will be referred to from this point forward, is shown applied to the ${ }^{252} \mathrm{Cf}$ alone case in Fig. A-7.

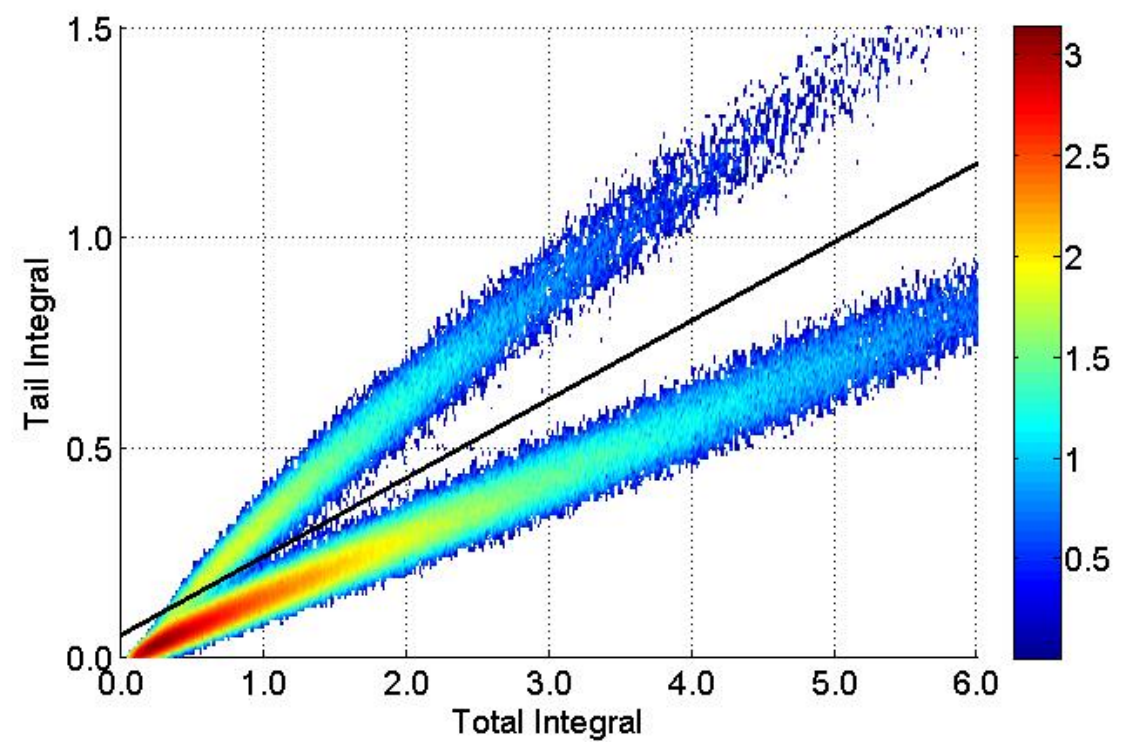

Figure A-7. Discrimination line applied to the ${ }^{252} \mathrm{Cf}$ alone case which is the lowest gamma-ray fluence scenario. The color scale measures the density of pulses in each region of the plot.

\section{Results}

There are several ways to evaluate the effectiveness of the PSD technique depending on the application of the measurement. In evaluating the discrimination method itself, comparing the neutron pulse-height distributions for various $\gamma / \mathrm{n}$ ratios allows for quantification and visualization of the misclassification of gamma-rays.

\subsection{Neutron Pulse-Height Distributions}

Comparing the neutron pulse-height distributions (PHDs) allows one to observe where gamma-ray misclassification is most frequently occurring in light-output space. Figures A-8 and A-9 show the measured neutron PHDs for the ${ }^{252} \mathrm{Cf}$ source alone, and the ${ }^{252} \mathrm{Cf}$ measured in combination with the 
${ }^{137} \mathrm{Cs}$ source placed at 2.5 to $175 \mathrm{~cm}$. By looking at deviation from the ${ }^{252} \mathrm{Cf}$ alone case as a function of keVee bin, it is possible to see which light-output ranges are subject to the largest errors. The results are produced by analyzing the data with the optimal PSD curve found in section 3 and a threshold of $50 \mathrm{keVee}$.

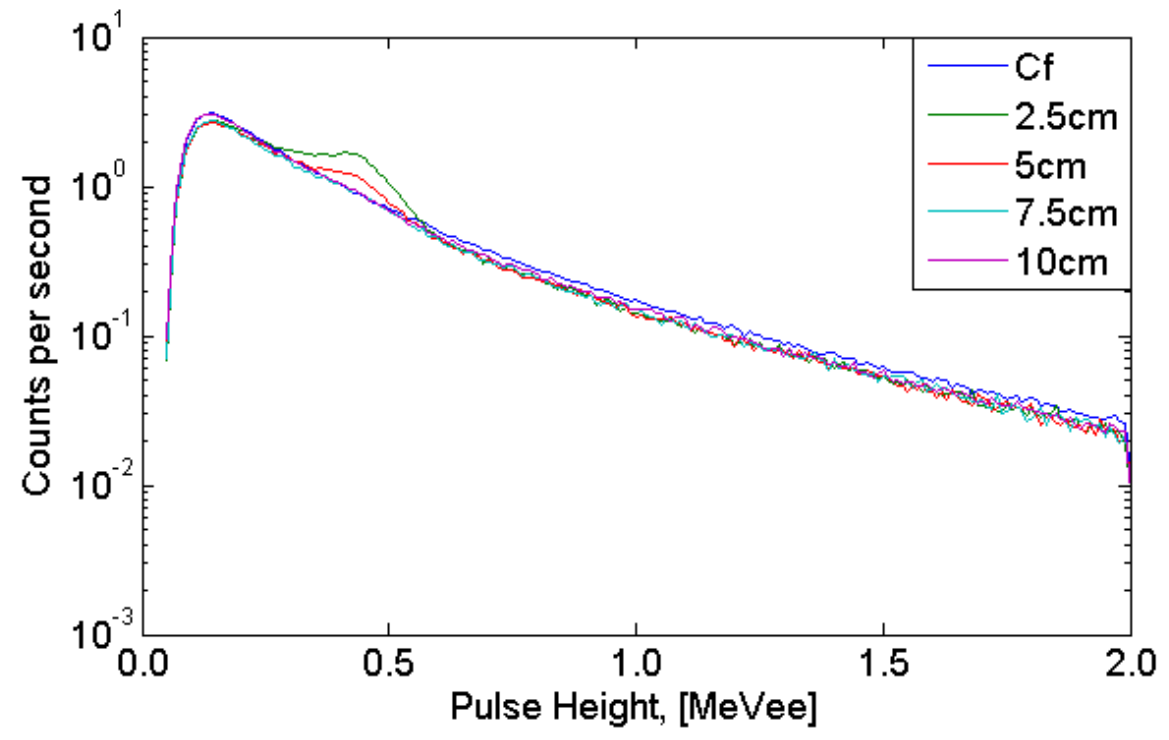

Figure A-8. Neutron PHDs for $2.5 \mathrm{~cm}$ through $10 \mathrm{~cm}{ }^{137} \mathrm{Cs}$ distances from the detector. The closest-range distances of $2.5 \mathrm{~cm}$ and $5 \mathrm{~cm}$ show a Compton edge from misclassified gammas, likely from missed double pulses.

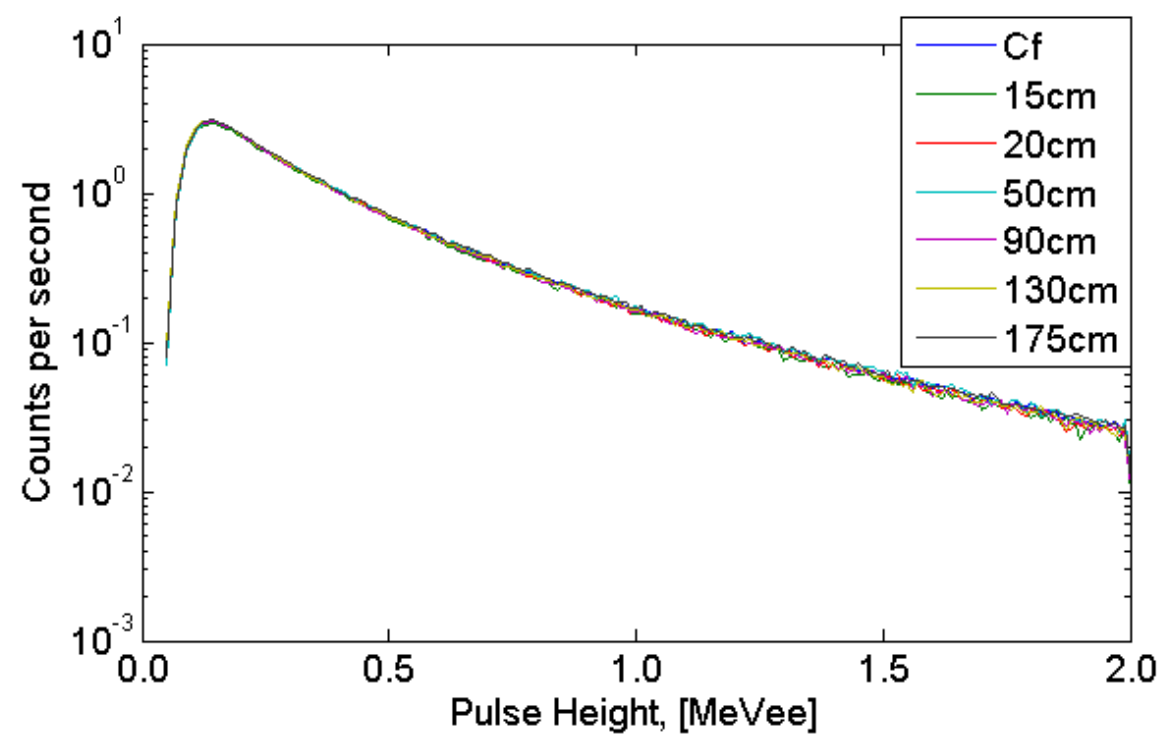

Figure A-9. Neutron PHDs for $15 \mathrm{~cm}$ through $175 \mathrm{~cm}$ distances from the detector. These distances show close agreement with the ${ }^{252} \mathrm{Cf}$ alone case. 
The ${ }^{137} \mathrm{Cs}$ Compton edge is apparent when the ${ }^{137} \mathrm{Cs}$ is within $5 \mathrm{~cm}$ of the detector. This effect occurs because misclassified gamma-rays are contributing to the distribution. The misclassification comes from the PSD and double pulses that are not eliminated in the analysis. It should also be noted that the PHDs for the various distances fall below the ${ }^{252} \mathrm{Cf}$ alone case by approximately $10 \%$ because there is a loss of neutrons from the double pulse effect and misclassification. As the ${ }^{137} \mathrm{Cs}$ count rate increases, more neutrons will be detected closely in time with gamma-rays causing them to be eliminated as double pulses. This effect is not a result of PSD itself and cannot be avoided for the current PSD approach with a high count rate while maintaining a low threshold.

To evaluate the deviation from the ${ }^{252} \mathrm{Cf}$ alone case for each pulse-height bin, the percent error is calculated as a function of pulse height. The cases with the more noticeable Compton edges are shown in Fig. A-10. The $15 \mathrm{~cm}-175 \mathrm{~cm}$ cases have the best agreement and the two extremes are shown in Fig. A-11.

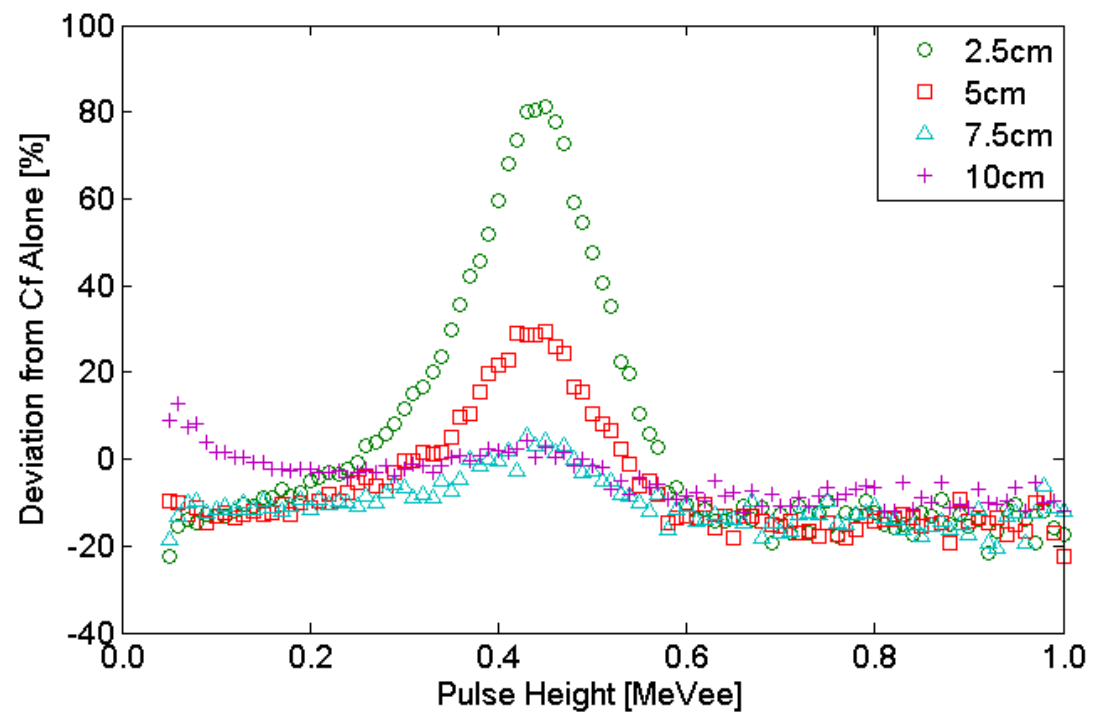

Figure A-10. Percent deviation of the various ${ }^{252} \mathrm{Cf}$ and ${ }^{137} \mathrm{Cs}$ experiments compared to the ${ }^{252} \mathrm{Cf}$ alone case, for the $2.5 \mathrm{~cm}$ through $10 \mathrm{~cm}$ cases. 


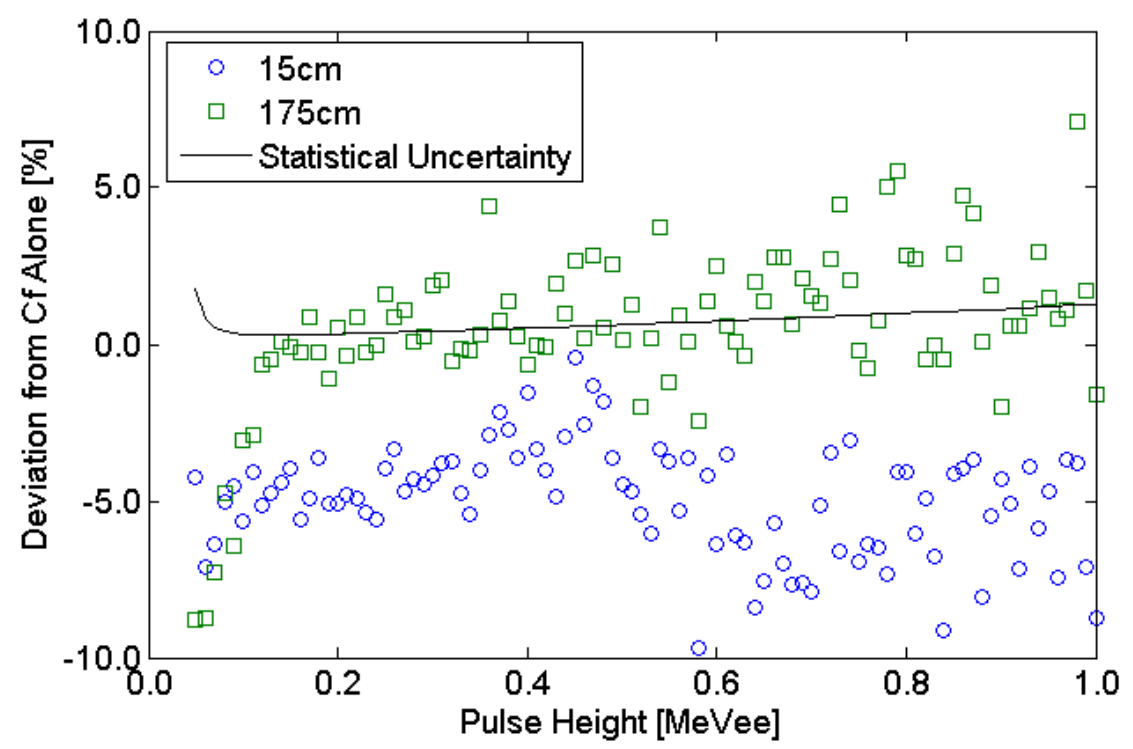

Figure A-11. Percent deviation of the various ${ }^{252} \mathrm{Cf}$ and ${ }^{137} \mathrm{Cs}$ experiments compared to the ${ }^{252} \mathrm{Cf}$ alone case, for the $15 \mathrm{~cm}$ and $175 \mathrm{~cm}$ cases.

The error values in the $2.5 \mathrm{~cm}-10 \mathrm{~cm}$ cases make evident the competing factors of the Compton edge and the neutron loss from double pulses. The neutron count rate is falsely inflated by gammarays arriving within a small window of one another and creating double pulses that are misclassified as neutrons. The count rate is also falsely deflated by the loss of neutrons which are detected shortly after a gamma-ray and are then rejected as double pulses. The agreement in the further (15- 175 $\mathrm{cm}$ ) cases is very good with values within $10 \%$ for the $0.05 \mathrm{MeVee}$ to $1 \mathrm{MeVee}$ range.

\subsection{Counting Statistics}

As the distance between the ${ }^{137} \mathrm{Cs}$ source and the detector is decreased, the neutron count rate will undergo a slight decrease because of an increased probability of pileup and deadtime losses. The neutron count rate is determined for all cases by summing over the neutron PHDs shown in section 4.1. By adding the counts per second observed in each pulse-height bin, we obtain the total measured neutron counts per second which is directly dependent on the PSD.

Some misclassification occurs despite implementation of PSD, so there is a varying amount of deviation from the expected value as seen in Fig. A-12. 


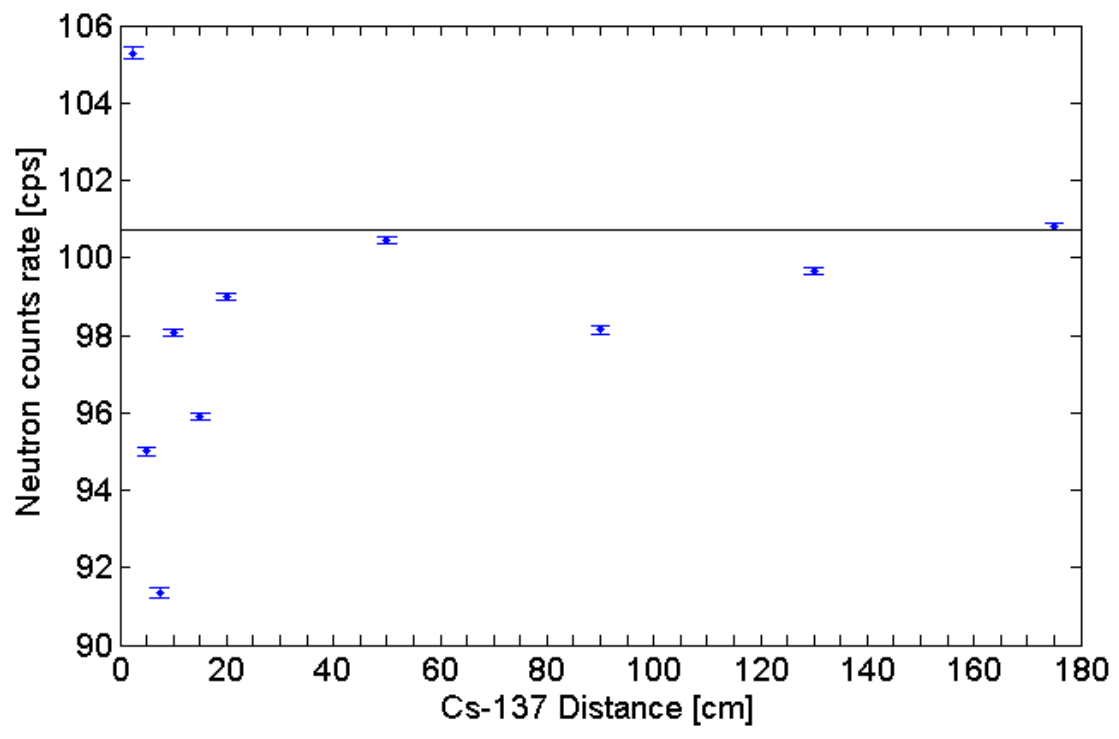

Figure A-12. Neutron count rate deviation from the ${ }^{252} \mathrm{Cf}$ alone case, with statistical uncertainties shown as standard error. The horizontal line shows where the values should be to match the ${ }^{252} \mathrm{Cf}$ alone case.

It would be expected that there would be the highest deviation where the $\gamma / \mathrm{n}$ ratio is highest, i.e. when ${ }^{137} \mathrm{Cs}$ is closest to the detector. Because of the competing factors discussed in section 3.1, we instead see the largest deviation at the $7.5 \mathrm{~cm}$ distance. The general trend is under-prediction of neutron count rate. This is expected because of the loss of neutrons through conservative doublepulse cleaning. The amount of deviation from the ${ }^{252} \mathrm{Cf}$ alone case count rate varies and causes the count rates to be scattered because of the competing factors. The exception to the under-prediction is the first data point at $2.5 \mathrm{~cm}$. At this distance, the gamma-ray emission from the ${ }^{137} \mathrm{Cs}$ source is sufficiently strong that the small probability of gamma-rays being misclassified as neutrons from PSD and double pulses will outweigh the neutrons eliminated as double pulses.

Table A-I gives percent error values for all distances along with the theoretical and measured $\gamma / \mathrm{n}$ ratios. The theoretical values are calculated using emission statistics only, and not accounting for energy-dependent detector efficiency. The percent errors are calculated as $\left(\mathrm{R}_{n}{ }^{\mathrm{Cf}+\mathrm{Cs}}-\mathrm{R}_{\mathrm{n}}{ }^{\mathrm{Cf}}\right) / \mathrm{R}_{\mathrm{n}}{ }^{\mathrm{Cf}}$ where $\mathrm{R}_{n}{ }^{\mathrm{Cf}+\mathrm{Cs}}$ is the sum of the time-normalized neutron PHDs of each independent distance measurement, and $\mathrm{R}_{\mathrm{n}}{ }^{\mathrm{Cf}}$ is the sum for the ${ }^{252} \mathrm{Cf}$ alone case. 
Table A-I. Percent error as a function of distance.

\begin{tabular}{cccc}
\hline Distance [cm] & $\begin{array}{c}\text { Theoretical } \\
\gamma / \text { n Ratio }\end{array}$ & $\begin{array}{c}\text { Measured } \\
\gamma / \text { n Ratio }\end{array}$ & Error [\%] \\
\hline 2.5 & 770.0 & 645.4 & 4.5 \\
5 & 352.1 & 480.3 & -5.7 \\
7.5 & 189.2 & 303.5 & -9.3 \\
10 & 116.5 & 208.8 & -2.6 \\
15 & 57.7 & 118.4 & -4.8 \\
20 & 35.5 & 73.3 & -1.7 \\
50 & 10.5 & 19.0 & -0.3 \\
90 & 7.1 & 9.3 & -2.6 \\
130 & 6.3 & 7.7 & -1.1 \\
175 & 6.0 & 6.5 & 0.1 \\
252 Cf Alone & 5.6 & 5.0 & -- \\
\hline
\end{tabular}

The percent errors are all a few percent of the ${ }^{252} \mathrm{Cf}$ alone case and all provide good approximations of the neutron count rate, off by a maximum of 10 neutrons per second out of 100 .

\section{Conclusions}

The PSD method employed in this work effectively discriminates gamma-rays at the majority of pulse heights and for all lower $\gamma / \mathrm{n}$ ratios. The discrimination is done using a threshold of $50 \mathrm{keVee}$, considerably lower than thresholds used in previous works. When the $\gamma / \mathrm{n}$ ratio is increased over 150 by placing the gamma-ray source very close to the detector face, the discrimination is less effective at eliminating gamma rays. To evaluate this effect we measured neutron PHDs for a wide range of $\gamma / \mathrm{n}$ ratios. The deviation of the $\mathrm{PHDs}$ to the control case of the ${ }^{252} \mathrm{Cf}$ source alone was systematically evaluated. Sources of error include the effect of gamma-rays being misclassified as neutrons because of double pulses and incorrect pulse identification through the PSD method. The neutron PHDs do follow the expected distribution for most $\gamma / \mathrm{n}$ ratios, and the neutron count rates are all within $10 \%$ of the count rate from ${ }^{252} \mathrm{Cf}$ alone, most within a few percent. These results show that liquid scintillators are a viable option for spent fuel measurement if the particular applications can sacrifice some neutron detection efficiency for the benefit of the fast-timing offered by these detectors. 


\section{Appendix B. Initial Operating Parameters for SFL4}

Spent Fuel Library 4 was the "mystery" library, with a variety of assemblies whose parameters were unknown to those using the library at the time of use. DDSI simulations were performed with the SFL4 library assemblies and results were analyzed without any prior knowledge of what types of assemblies were present. The actual parameters used to create each assembly in the library are given here. 


\begin{tabular}{|c|c|c|c|c|c|}
\hline $\begin{array}{c}\text { SFL4 } \\
\text { case }\end{array}$ & IE & CT & BU & $\begin{array}{c}\text { Downtime } \\
\text { Between } \\
\text { Cycles (days) }\end{array}$ & Notes \\
\hline 1 & 4 & 20 & 21.5 & 1125.72 & \\
\hline 2 & 4 & 1 & 40 & 1125.72 & \\
\hline 3 & 2.6 & 5 & 25.3 & 30 & \\
\hline 4 & 2.6 & 80 & 10.9 & 30 & \\
\hline 5 & 2.8 & 50 & 16.8 & 30 & \\
\hline 6 & 2.8 & 1 & 27.4 & 30 & \\
\hline 7 & 3.4 & 20 & 22.3 & 30 & \\
\hline 8 & 3.4 & 50 & 34.2 & 30 & \\
\hline 9 & 3.6 & 5 & 19.6 & 30 & \\
\hline 10 & 3.6 & 80 & 36.1 & 30 & \\
\hline 11 & 3.8 & 20 & 14.1 & 30 & \\
\hline 12 & 3.8 & 5 & 37.9 & 30 & \\
\hline 13 & 4 & 80 & 21.3 & 30 & Control rods present in cycle 1 \\
\hline 14 & 4 & 50 & 37.8 & 30 & Control rods present in cycle 1 \\
\hline 15 & 4 & 1 & 21.3 & 30 & \\
\hline 16 & 4 & 80 & 39.9 & 30 & \\
\hline 17 & 4.2 & 5 & 22.3 & 30 & \\
\hline 18 & 4.2 & 1 & 42.8 & 30 & \\
\hline 19 & 3.2 & 20 & 19.7 & 30 & \\
\hline 20 & 3.2 & 50 & 31.7 & 30 & \\
\hline 21 & 4 & 5 & 21.4 & 562.86 & \\
\hline 22 & 4 & 80 & 39.9 & 562.86 & \\
\hline 23 & 4 & 50 & 21.5 & 2190 & 1434.5 days irr., 2190 CT, 1 yr irr. \\
\hline 24 & 4 & 1 & 40.3 & 2190 & 1434.5 days irr., $2190 \mathrm{CT}, 1$ yr irr. \\
\hline 25 & 4 & 5 & 18.4 & 0 & $1 / 8$ core simulation of all rods \\
\hline 26 & 4 & 20 & 35.6 & 0 & $1 / 8$ core simulation of all rods \\
\hline 27 & 4 & 1 & 21.3 & 30 & 40 void rods in center \\
\hline 28 & 4 & 1 & 21.3 & 30 & $40 \mathrm{DU}$ rods in center \\
\hline 29 & 4 & 1 & 21.3 & 30 & $40 \mathrm{NU}$ rods in center \\
\hline 30 & 4 & 1 & 21.3 & 30 & $20 \mathrm{NU}$ rods equally distributed \\
\hline 31 & 4 & 1 & 21.3 & 30 & 20 DU rods equally distributed \\
\hline 32 & 4 & 1 & 21.3 & 30 & $40 \mathrm{NU}$ rods equally distributed \\
\hline 33 & 4 & 1 & 21.3 & 30 & 40 DU rods equally distributed \\
\hline 34 & 4 & 1 & 21.3 & 30 & 40 void rods equally distributed \\
\hline 35 & 4 & 5 & 28 & 30 & \\
\hline \multirow[t]{2}{*}{36} & 4 & 5 & 28 & 0 & $1 / 8$ core simulation of all rods \\
\hline & & & & & Partial Defect Cases in bold \\
\hline
\end{tabular}




\section{Appendix C. Example MCNP Input File}

\section{LANL 15x15 Fresh Fuel Assembly with DDSI Equipment}

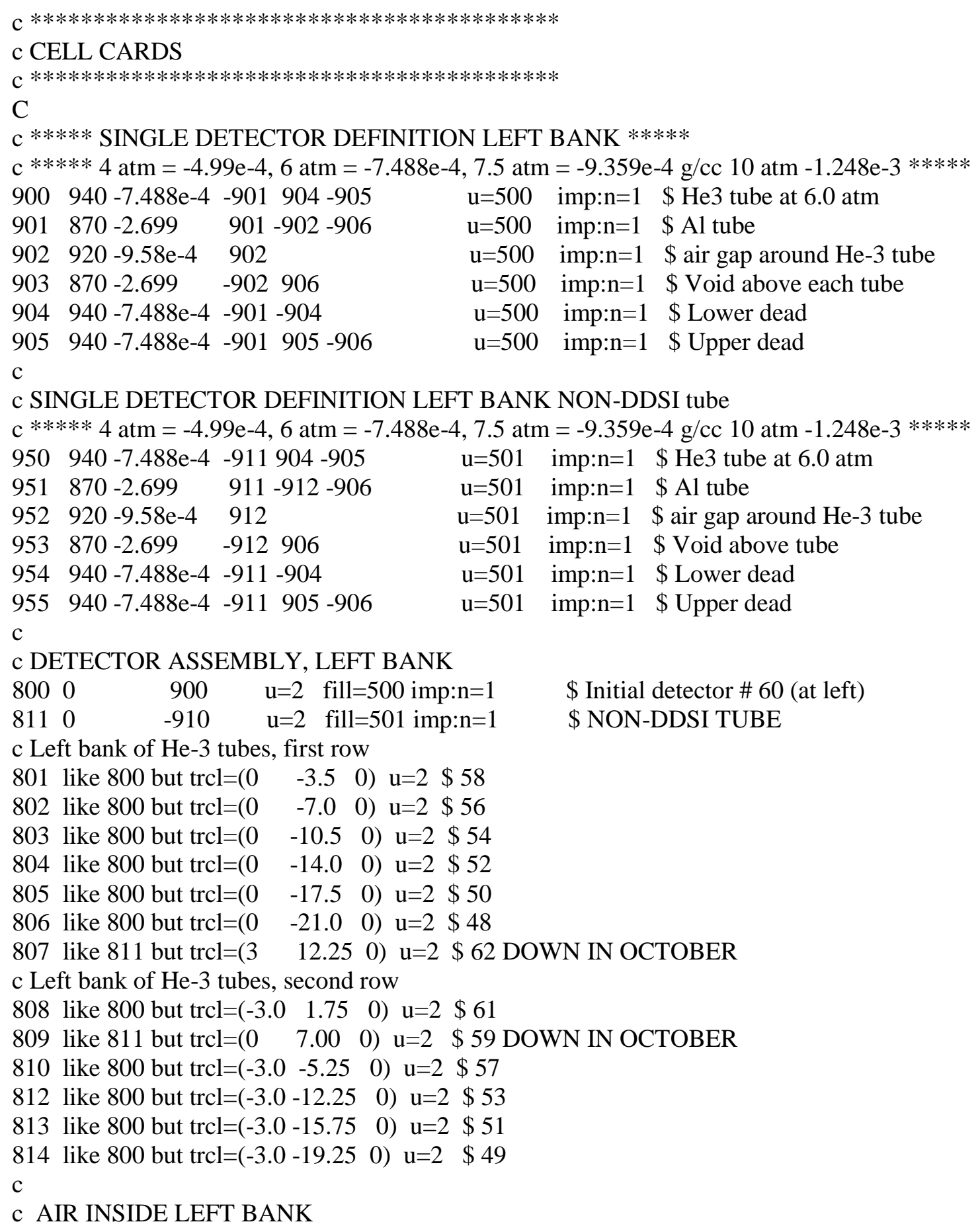




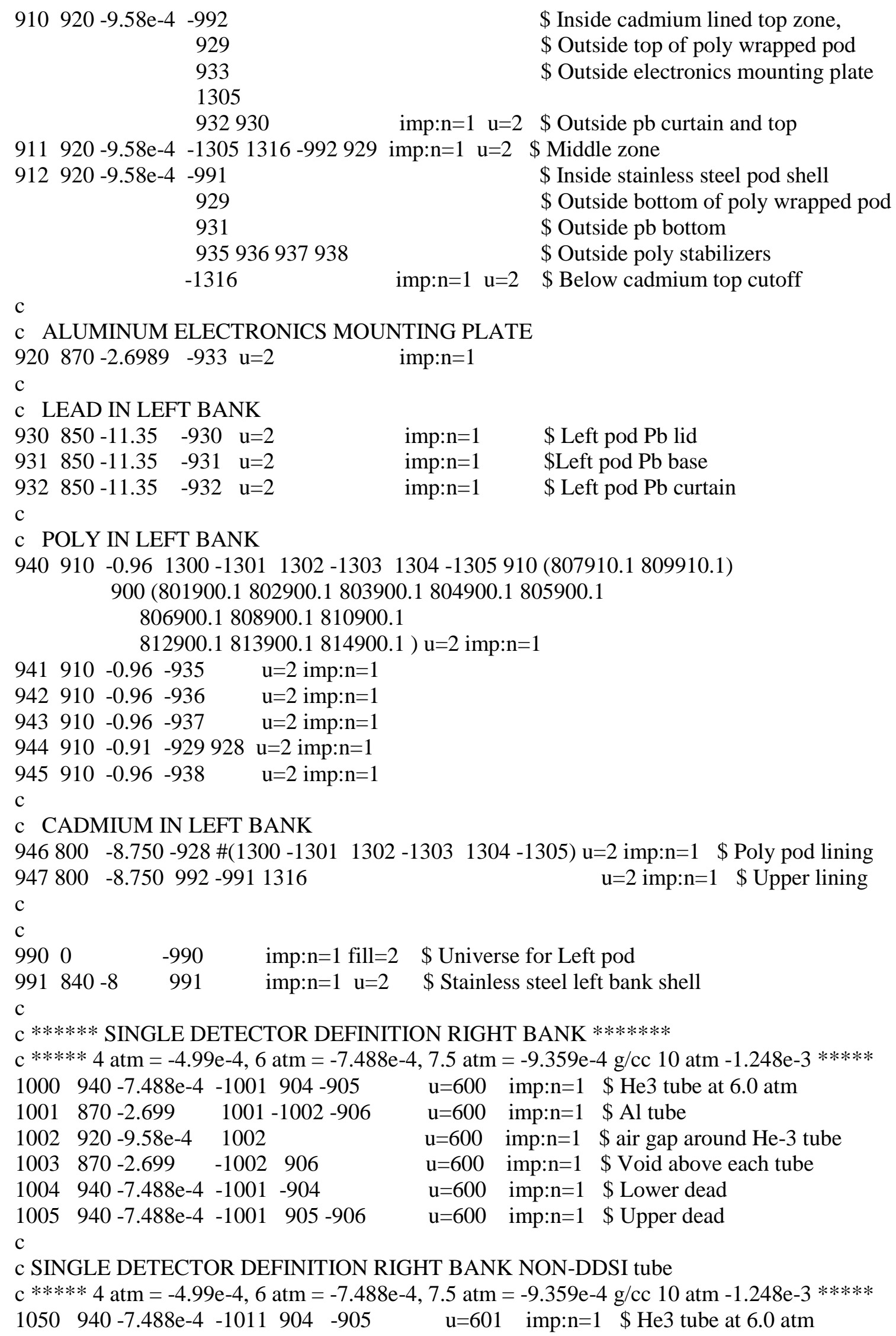




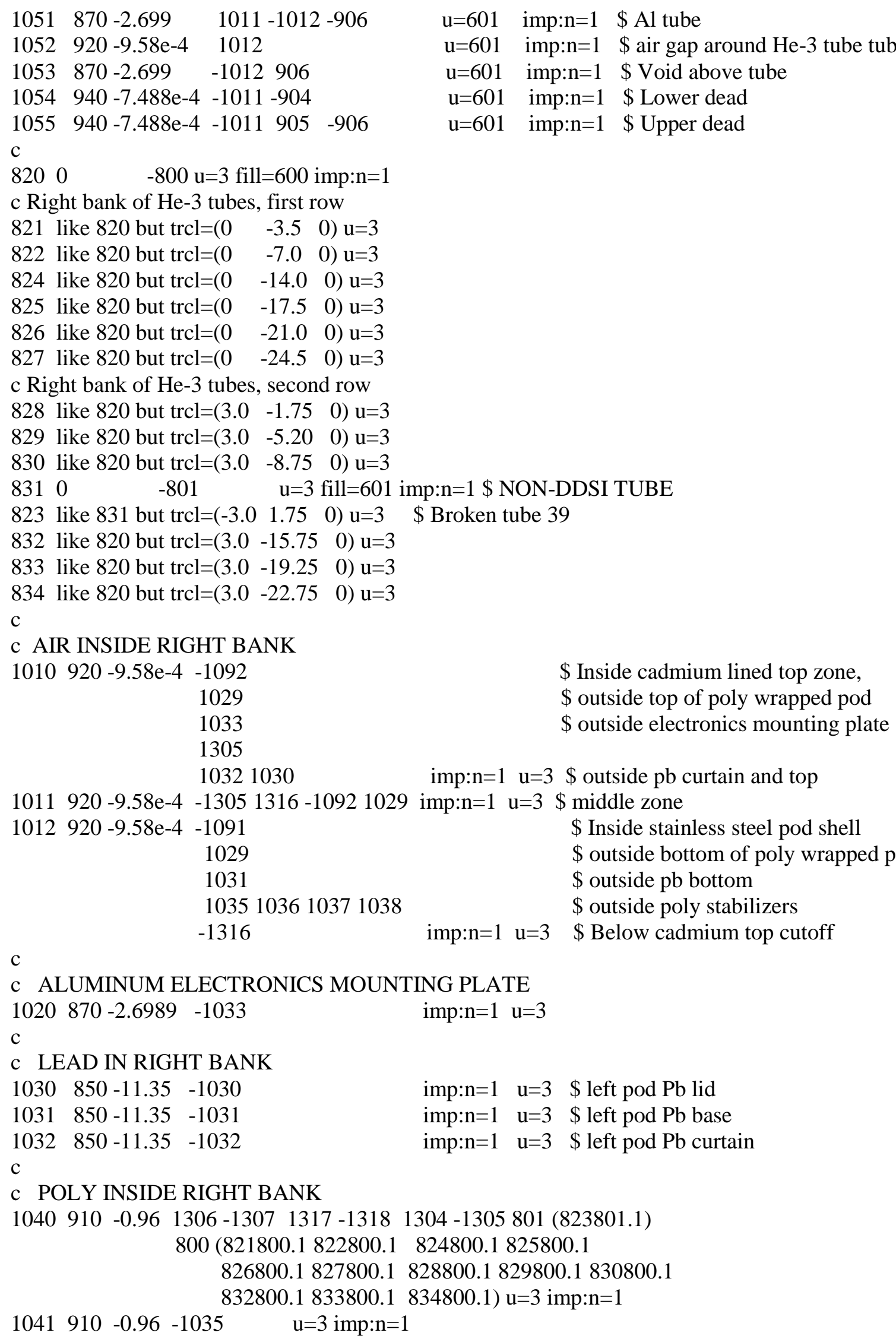




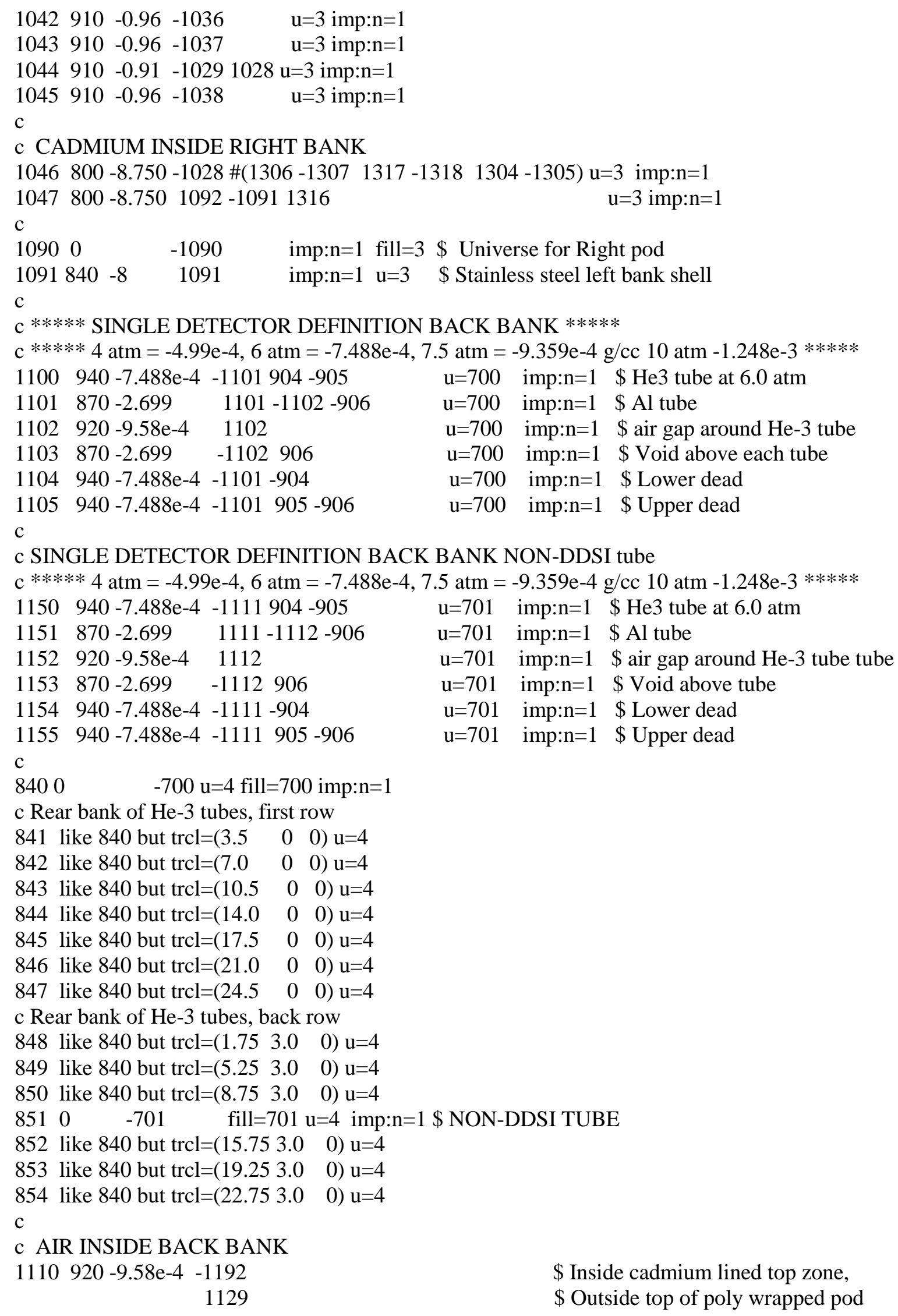

\$ Inside cadmium lined top zone, $\$$ Outside top of poly wrapped pod 
$11321130 \quad$ imp:n=1 u=4 \$ Outside pb curtain and top

$$
1111920-9.58 \mathrm{e}-4 \quad-13051316-11921129 \text { imp:n=1 u=4 }
$$

$1112920-9.58 \mathrm{e}-4-1191$

1129

1131

1135113611371138

$-1316 \quad$ imp:n=1 u=4
\$ Inside stainless steel pod shell \$ Outside bottom of poly wrapped pod $\$$ Outside pb bottom $\$$ Outside poly stabilizers imp:n=1 u=4 \$ Below cadmium top cutoff

$\mathrm{c}$

c ALUMINUM ELECTRONICS MOUNTING PLATE $1120870-2.6989-1133 \quad \mathrm{u}=4 \quad \mathrm{imp}: \mathrm{n}=1$

c

c LEAD IN BACK BANK

$1130850-11.35 \quad-1130 \quad \mathrm{u}=4 \quad$ imp:n=1 \$ Left pod $\mathrm{Pb}$ lid

$1131850-11.35-1131 \quad \mathrm{u}=4 \quad$ imp:n=1 \$ Left pod $\mathrm{Pb}$ base

$1132850-11.35-1132 \quad \mathrm{u}=4 \quad$ imp:n=1 \$ Left pod $\mathrm{Pb}$ curtain

c

c POLY INSIDE BACK BANK

$\begin{array}{lllllll}1140 & 910 & -0.96 & 1308 & -1309 & 1310-1311 & 1304-1305701\end{array}$

700 (841700.1 842700.1843700.1844700.1845700.1

846700.1847700 .1848700 .1849700 .1850700 .1

$852700.1853700 .1854700 .1) \mathrm{u}=4 \mathrm{imp}: n=1$

$1141910-0.96-1135 \quad \mathrm{u}=4 \mathrm{imp}: \mathrm{n}=1$

$1142910-0.96-1136 \quad \mathrm{u}=4 \mathrm{imp}: \mathrm{n}=1$

$1143910-0.96-1137 \quad \mathrm{u}=4 \mathrm{imp}: \mathrm{n}=1$

$1144910-0.91-11291128-1305 \mathrm{u}=4 \mathrm{imp}: \mathrm{n}=1$

$1145910-0.96-1138 \quad \mathrm{u}=4$ imp:n=1

c

c CADMIUM INSIDE BACK BANK

$1146800-8.650-1128$ \#(1308 -1309 $1310-1311 \quad 1304-1305)$ u=4 imp:n=1

$1147800-8.7501192-11911316 \quad u=4$ imp:n=1

c

$11900 \quad-1190 \quad$ imp:n=1 fill=4 \$ Universe for Back pod

$1191840-8 \quad 1191 \quad$ imp:n=1 u=4 \$ Stainless steel left bank shell

c

c $* * * * *$ SINGLE DETECTOR DEFINITION FRONT BANK $* * * * *$

$\mathrm{c} * * * * * 4 \mathrm{~atm}=-4.99 \mathrm{e}-4,6 \mathrm{~atm}=-7.488 \mathrm{e}-4,7.5 \mathrm{~atm}=-9.359 \mathrm{e}-4 \mathrm{~g} / \mathrm{cc} 10 \mathrm{~atm}-1.248 \mathrm{e}-3 * * * * *$

$1200940-7.488 \mathrm{e}-4 \quad-1201904 \quad-905 \quad \mathrm{u}=800 \quad \mathrm{imp}: \mathrm{n}=1 \quad \$ \mathrm{He} 3$ tube at 6.0 atm

$1201870-2.699 \quad 1201-1202-906 \quad \mathrm{u}=800 \quad$ imp:n=1 \$ Al tube

$1202 \quad 920-9.58 \mathrm{e}-4 \quad 1202$

$1203 \quad 870-2.699 \quad-1202906$

$1204940-7.488 \mathrm{e}-4-1201-904$

$\mathrm{u}=800$ imp:n=1 \$ Air gap around He-3 tube

$1205940-7.488 \mathrm{e}-4 \quad-1201905-906$

$\mathrm{u}=800$ imp:n=1 \$Void above each tube

$\mathrm{u}=800$ imp:n=1 \$Lower dead

$\mathrm{c}$

c SINGLE DETECTOR DEFINITION FRONT BANK - NON DDSI tube

$\mathrm{c} * * * * * 4 \mathrm{~atm}=-4.99 \mathrm{e}-4,6 \mathrm{~atm}=-7.488 \mathrm{e}-4,7.5 \mathrm{~atm}=-9.359 \mathrm{e}-4 \mathrm{~g} / \mathrm{cc} 10 \mathrm{~atm}-1.248 \mathrm{e}-3 * * * * *$

$1250940-7.488 \mathrm{e}-4-1211904-905 \quad \mathrm{u}=801 \quad \mathrm{imp}: \mathrm{n}=1 \quad \$ \mathrm{He} 3$ tube at $6.0 \mathrm{~atm}$

$1251 \quad 870-2.699 \quad 1211-1212-906$

$1252 \quad 920-9.58 \mathrm{e}-4 \quad 1212$

$\begin{array}{llll}1253 & 870-2.699 & -1212906\end{array}$

$\mathrm{u}=801$ imp:n=1 \$Al tube

$\mathrm{u}=801$ imp:n=1 \$ Air gap around He-3 tube

$\mathrm{u}=801$ imp:n=1 \$ Void above tube 


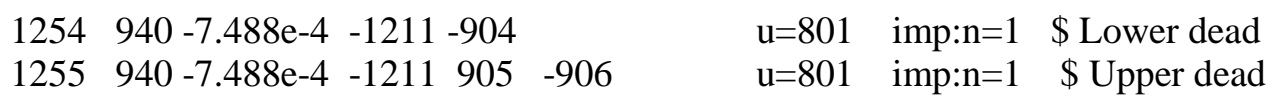

c

$860 \quad 0 \quad-600 \mathrm{u}=5$ fill $=800 \mathrm{imp}: \mathrm{n}=1$

c FRONT BANK OF HE-3 TUBES, FIRST ROW

861 like 860 but $\operatorname{trcl}=\left(\begin{array}{lll}3.5 & 0 & 0\end{array}\right) \mathrm{u}=5$

862 like 860 but $\operatorname{trcl}=\left(\begin{array}{lll}7.0 & 0 & 0\end{array}\right) \mathrm{u}=5$

863 like 860 but $\operatorname{trcl}=\left(\begin{array}{lll}10.5 & 0 & 0\end{array}\right) \mathrm{u}=5$

864 like 860 but $\operatorname{trcl}=(14.0 \quad 0 \quad 0) \mathrm{u}=5$

865 like 860 but $\operatorname{trcl}=\left(\begin{array}{lll}17.5 & 0 & 0\end{array}\right) \mathrm{u}=5$

866 like 860 but $\operatorname{trcl}=(21.0 \quad 0 \quad 0) \mathrm{u}=5$

867 like 860 but $\operatorname{trcl}=(24.5 \quad 0 \quad 0) \mathrm{u}=5$

c FRONT BANK OF HE-3 TUBES, BACK ROW

868 like 860 but $\operatorname{trcl}=\left(\begin{array}{lll}1.75 & -3.0 & 0\end{array}\right) \mathrm{u}=5$

869 like 860 but $\operatorname{trcl}=\left(\begin{array}{lll}5.25 & -3.0 & 0\end{array}\right) \mathrm{u}=5$

870 like 860 but $\operatorname{trcl}=\left(\begin{array}{lll}8.75 & -3.0 & 0\end{array}\right) \mathrm{u}=5$

$8710 \quad-1210$ fill=801 $\mathrm{u}=5 \mathrm{imp}: \mathrm{n}=1$ \$ REMOVE MIDDLE TUBE

872 like 860 but $\operatorname{trcl}=\left(\begin{array}{lll}15.75 & -3.0 & 0\end{array}\right) \mathrm{u}=5$

873 like 860 but $\operatorname{trcl}=(19.25-3.0 \quad 0) \mathrm{u}=5$

874 like 860 but $\operatorname{trcl}=(22.75-3.0 \quad 0) \mathrm{u}=5$

c

c AIR INSIDE FRONT BANK

$1210920-9.58 \mathrm{e}-4 \quad-1292$

1229

1233

1305

12321230

\$ Inside cadmium lined top zone,

$\$$ Outside top of poly wrapped pod

\$ Outside electronics mounting plate

1211920 -9.58e-4 -1305 $1316-12921229$ imp:n=1 u=5 \$ Middle zone

$1212920-9.58 \mathrm{e}-4-1291$

1229

1231

1235123612371238

$-1316 \quad$ imp:n=1 u=5

\$ Inside stainless steel pod shell

\$ Outside bottom of poly wrapped pod

$\$$ Outside pb bottom

$\$$ Outside poly stabilizers

c

c ALUMINUM ELECTRONICS MOUNTING PLATE

$1220870-2.6989-1233 \quad \mathrm{u}=5 \quad$ imp: $n=1$

$\mathrm{c}$

c LEAD IN FRONT BANK

$1230 \quad 850-11.35 \quad-1230 \quad \mathrm{u}=5 \quad$ imp:n=1 \$ Left pod $\mathrm{Pb}$ lid

$1231850-11.35-1231 \quad \mathrm{u}=5 \quad$ imp:n=1 \$ Left pod $\mathrm{Pb}$ base

$1232850-11.35-1232 \quad \mathrm{u}=5 \quad$ imp: $\mathrm{n}=1 \quad$ \& Left pod $\mathrm{Pb}$ curtain

$\mathrm{C}$

c POLY INSIDE FRONT BANK

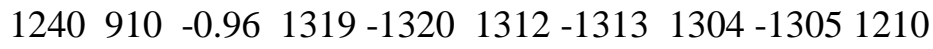

$600(861600.1862600 .1863600 .1864600 .1865600 .1$

866600.1867600 .1868600 .1869600 .1870600 .1

$872600.1873600 .1874600 .1) \mathrm{u}=5 \mathrm{imp}: \mathrm{n}=1$

$1241910-0.96-1235$

$1242910-0.96-1236$

$\mathrm{u}=5$ imp: $\mathrm{n}=1$

$\begin{array}{llll}1243 & 910 & -0.96 & -1237\end{array}$

$\mathrm{u}=5 \mathrm{imp}: \mathrm{n}=1$

$1244910-0.91-12291228$

$\mathrm{u}=5$ imp: $\mathrm{n}=1$

$\mathrm{u}=5 \mathrm{imp}: \mathrm{n}=1$ 


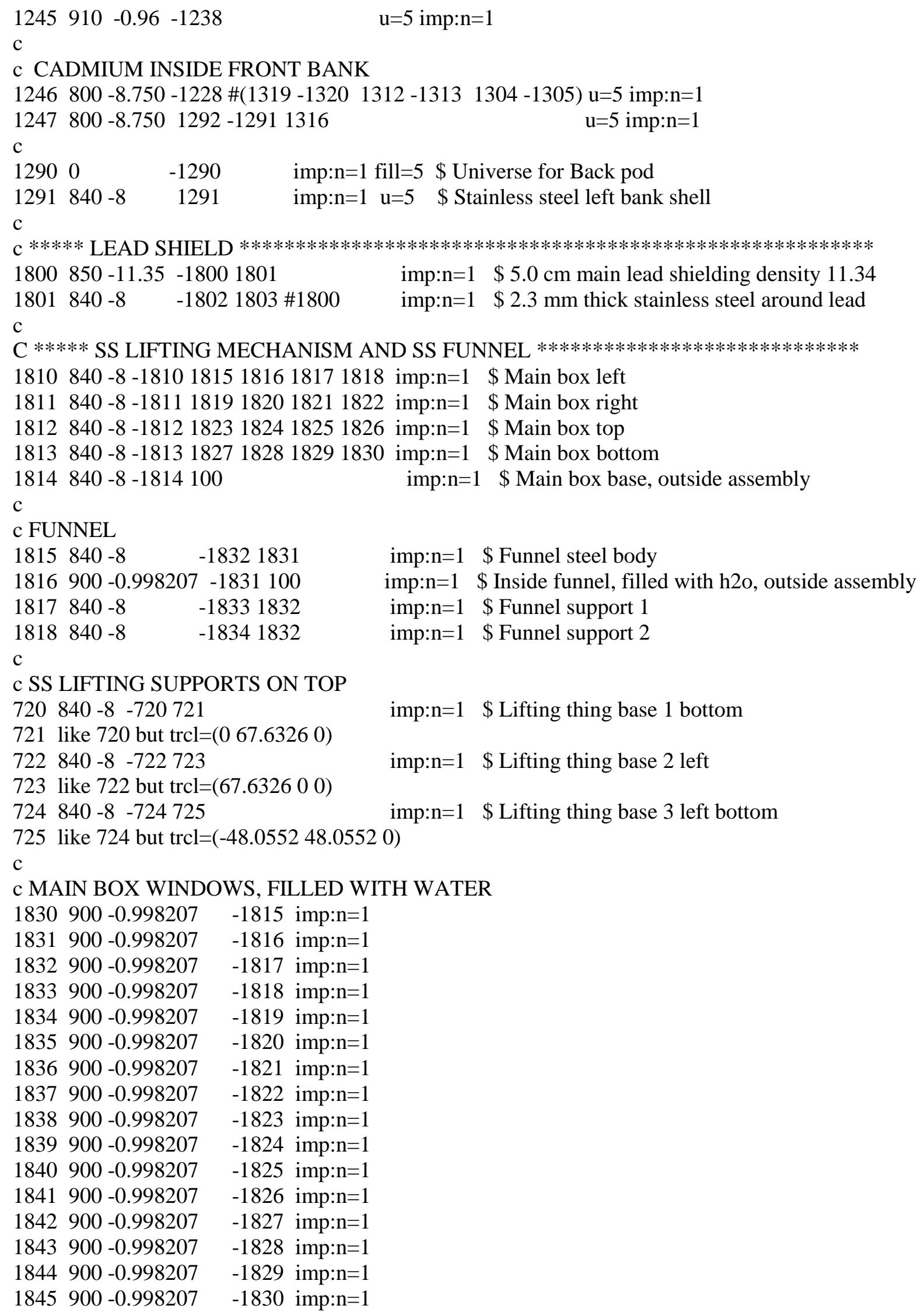

$724840-8 \quad-724725 \quad$ imp:n=1 \$ Lifting thing base 3 left bottom

imp:n=1 \$ Lifting thing base 1 bottom

imp:n=1 \$ Lifting thing base 2 left 


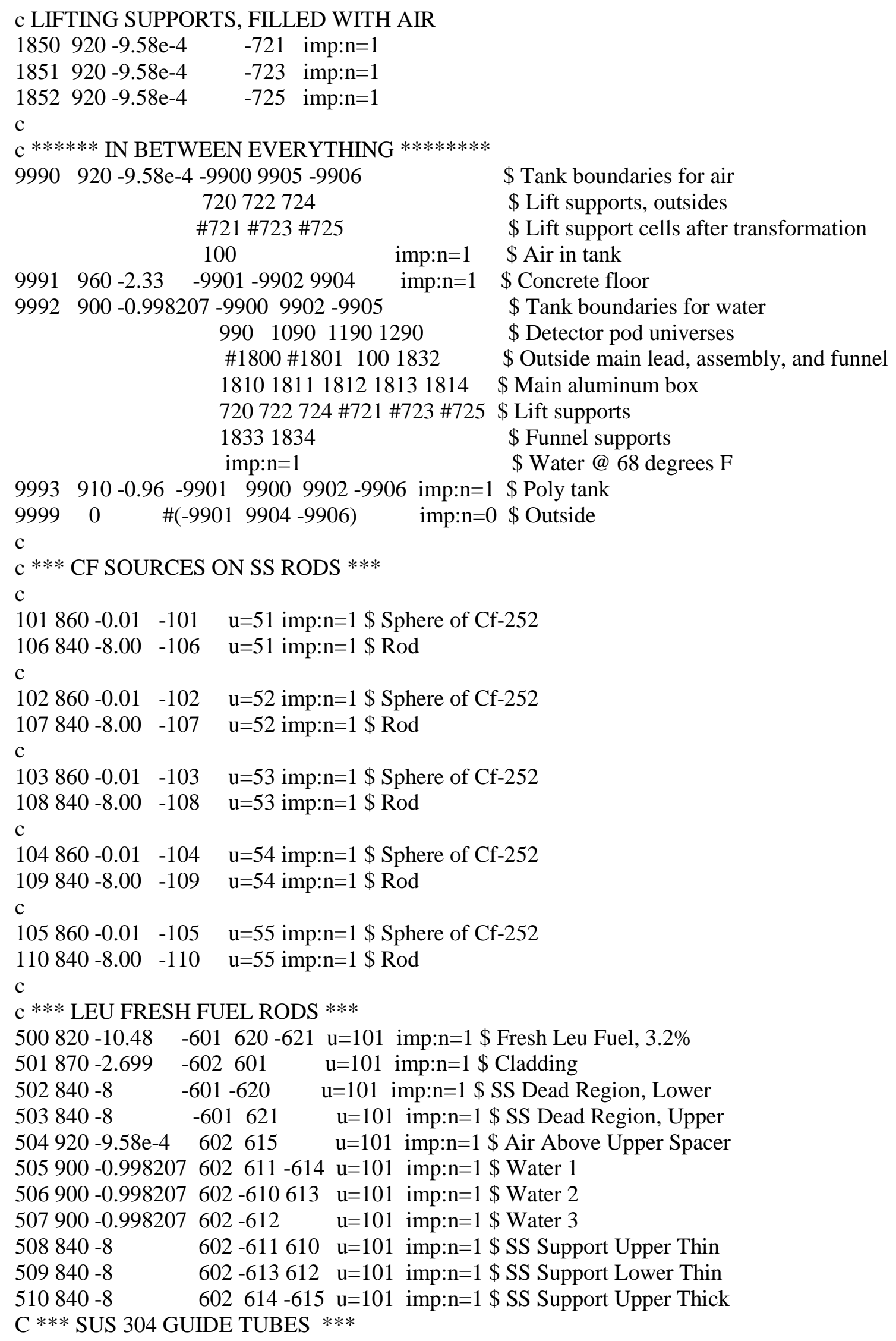




\begin{tabular}{|c|c|}
\hline & \\
\hline & \\
\hline & \\
\hline $40-8$ & $\mathrm{u}=50$ imp:n=1 \$ Guide Tube \\
\hline $4920-9.58 \mathrm{e}-4$ & $605615 \quad \mathrm{u}=50$ imp:n=1 \$ Air Above Upper Spacer \\
\hline $515900-0.998207$ & imp:n=1 \$ Water 1 \\
\hline $516900-0.998207$ & $605-610 \quad 613 u=50$ \\
\hline $517900-0.998207$ & imp:n=1 \$ Water 3 \\
\hline $518840-8$ & imp:n=1 \$ SS Support Upper Thin \\
\hline $519840-8$ & $605-613612 u=50$ imp:n=1 \$ SS Support Lower Thin \\
\hline $520840-8$ & imp:n=1 \$ SS Support Upper Thick \\
\hline$* * *$ SUS $304 \mathrm{GU}$ & JIDE TUBES , UNIVERSE $51 * * *$ \\
\hline $21900-0$ & $-604101106-624 u=51$ imp:n=1 \$ Inside Of Guide Tube, Water \\
\hline $522920-9.58 \mathrm{e}-4$ & $-604624 \quad \mathrm{u}=51$ imp:n=1 \$ Inside Of Guide Tube, Air \\
\hline $523840-8$ & $\mathrm{u}=51$ imp:n=1 \$ Guide Tube \\
\hline $524920-9.58 \mathrm{e}-4$ & $\mathrm{u}=51$ imp: $\mathrm{n}=1$ \$ Air Above Upper Spacer \\
\hline $525900-0.998207$ & $\mathrm{u}=51$ imp:n=1 \$ Water 1 \\
\hline $526900-0.998207$ & $\mathrm{u}=51$ imp:n=1 \$ Water 2 \\
\hline $527900-0.998207$ & $\mathrm{u}=51$ imp: $\mathrm{n}=1$ \$ Water 3 \\
\hline $528840-8$ & $\mathrm{u}=51$ imp:n=1 \$ SS Support Upper Thin \\
\hline $529840-8$ & $\mathrm{u}=51$ imp:n=1 \$ SS Support Lower Thin \\
\hline $530840-8$ & $\mathrm{u}=51$ imp:n=1 \$ SS Support Upper Thick \\
\hline C *** SUS $304 \mathrm{GU}$ & JIDE TUBES , UNIVERSE $52 * * *$ \\
\hline 8207 & $-604102107-624 u=52$ imp:n=1 \$ Inside O \\
\hline $8 \mathrm{e}-4$ & $-604624 \quad \mathrm{u}=52$ imp:n=1 \$ Inside Of Gu \\
\hline $533840-8$ & $\mathrm{u}=52$ imp:n=1 \$ Guide Tube \\
\hline $534920-9.58 \mathrm{e}-4$ & $\mathrm{u}=52$ imp: $\mathrm{n}=1$ \$ Air Above Upper Spacer \\
\hline $535900-0.998207$ & $\mathrm{u}=52$ imp: $\mathrm{n}=1$ \$ Water 1 \\
\hline $536900-0.998207$ & $\mathrm{u}=52$ imp:n=1 \$ Water 2 \\
\hline $537900-0.998207$ & $\mathrm{u}=52$ imp: $\mathrm{n}=1$ \$ Water 3 \\
\hline $538840-8$ & $\mathrm{u}=52$ imp: $\mathrm{n}=1$ \\
\hline $539840-8$ & $\mathrm{u}=52$ imp:n=1 \$ SS Support Lower Thin \\
\hline $540840-8$ & $\mathrm{u}=52$ imp:n=1 \$ SS Support Upper Thick \\
\hline C *** SUS $304 \mathrm{GU}$ & JIDE TUBES , UNIVERSE $53 * * *$ \\
\hline $541900-0.998207$ & $-604103108-624$ u=53 imp:n=1 \$ Inside Of Guide Tube, Water \\
\hline $542920-9.58 \mathrm{e}-4$ & $-604624 \quad \mathrm{u}=53$ imp:n=1 \$ Inside Of Guide Tube, Air \\
\hline $543840-8$ & $\mathrm{u}=53$ imp:n=1 $\$$ Guide Tube \\
\hline $544920-9.58 \mathrm{e}-4$ & $\mathrm{u}=53$ imp: $\mathrm{n}=1$ \$ Air Above Upper Spacer \\
\hline $545900-0.998207$ & $\mathrm{u}=53$ imp: $\mathrm{n}=1$ \$ Water 1 \\
\hline $546900-0.998207$ & $\mathrm{u}=53$ imp: $\mathrm{n}=1$ \$ Water 2 \\
\hline $547900-0.998207$ & $\mathrm{u}=53$ imp:n=1 \$ Water 3 \\
\hline $548840-8$ & $\mathrm{u}=53$ imp:n=1 \$ SS Support Upper Thin \\
\hline $0-8$ & $\mathrm{u}=53$ imp:n=1 \$ SS Support Lower Thin \\
\hline $550840-8$ & $605614-615 \quad \mathrm{u}=53$ imp:n=1 \$ SS Support Upper Thick \\
\hline $\mathrm{C} * * *$ SUS $304 \mathrm{GU}$ & JIDE TUBES UNIVERSE $54 * * *$ \\
\hline $551900-0.998207$ & $-604104109-624$ u=54 imp:n=1 \$ Inside Of Guide Tube, Water \\
\hline $552920-9.58 \mathrm{e}-4$ & $\mathrm{u}=54$ imp: $\mathrm{n}=1$ \$ Inside Of Guide Tube, Air \\
\hline $553840-8$ & $\mathrm{u}=54$ imp: $\mathrm{n}=1$ \$ Guide Tube \\
\hline $554920-9.58 \mathrm{e}-4$ & $\mathrm{u}=54$ imp: $\mathrm{n}=1$ \$ Air Above Upper Spacer \\
\hline $555900-0.998207$ & $\mathrm{u}=54$ imp: $\mathrm{n}=1 \$$ Water 1 \\
\hline $556900-0.998207$ & $\mathrm{u}=54$ imp: $\mathrm{n}=1$ \$ Water 2 \\
\hline $557900-0.998207$ & $\mathrm{u}=54$ imp:n=1 \$ Water 3 \\
\hline
\end{tabular}




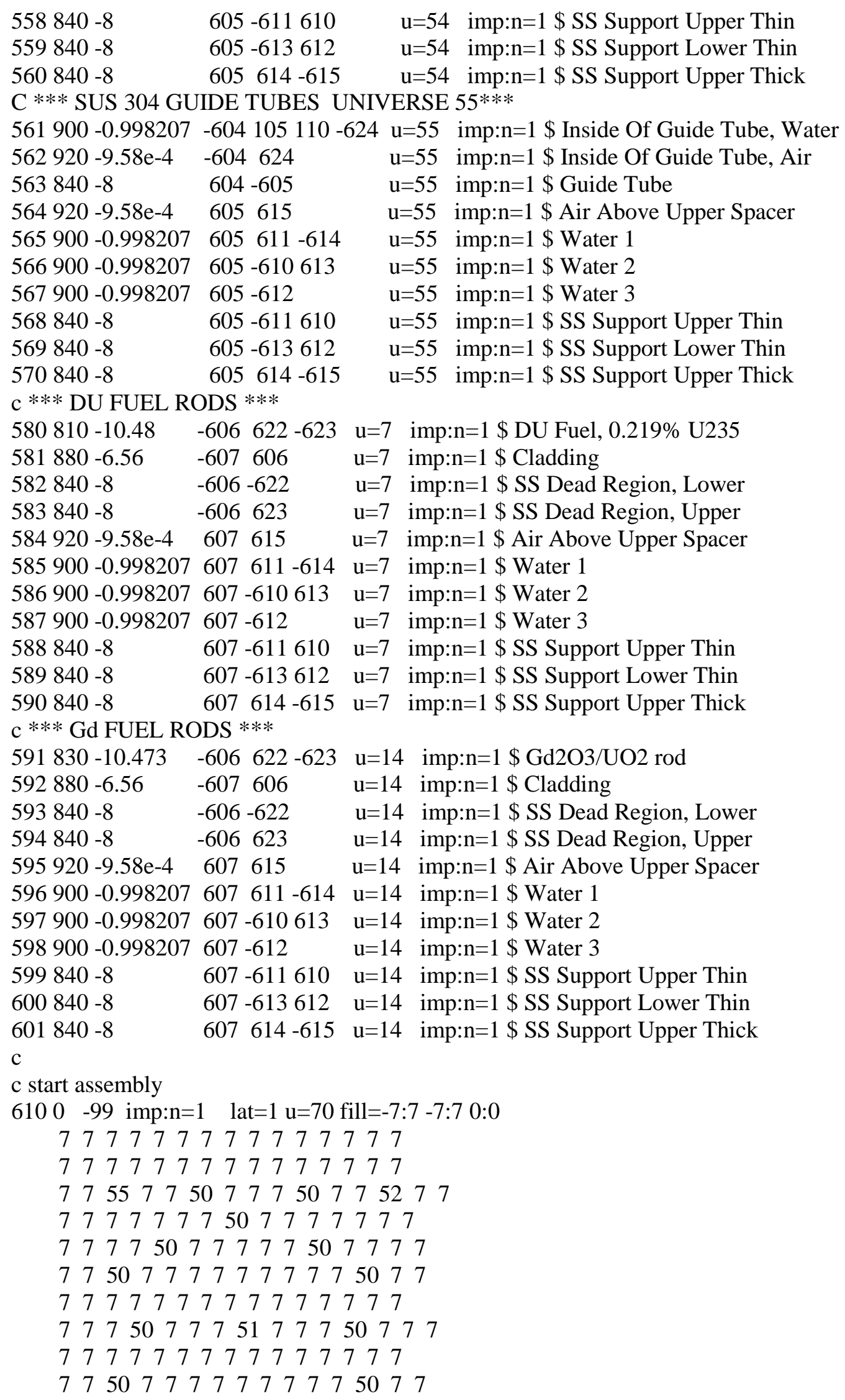




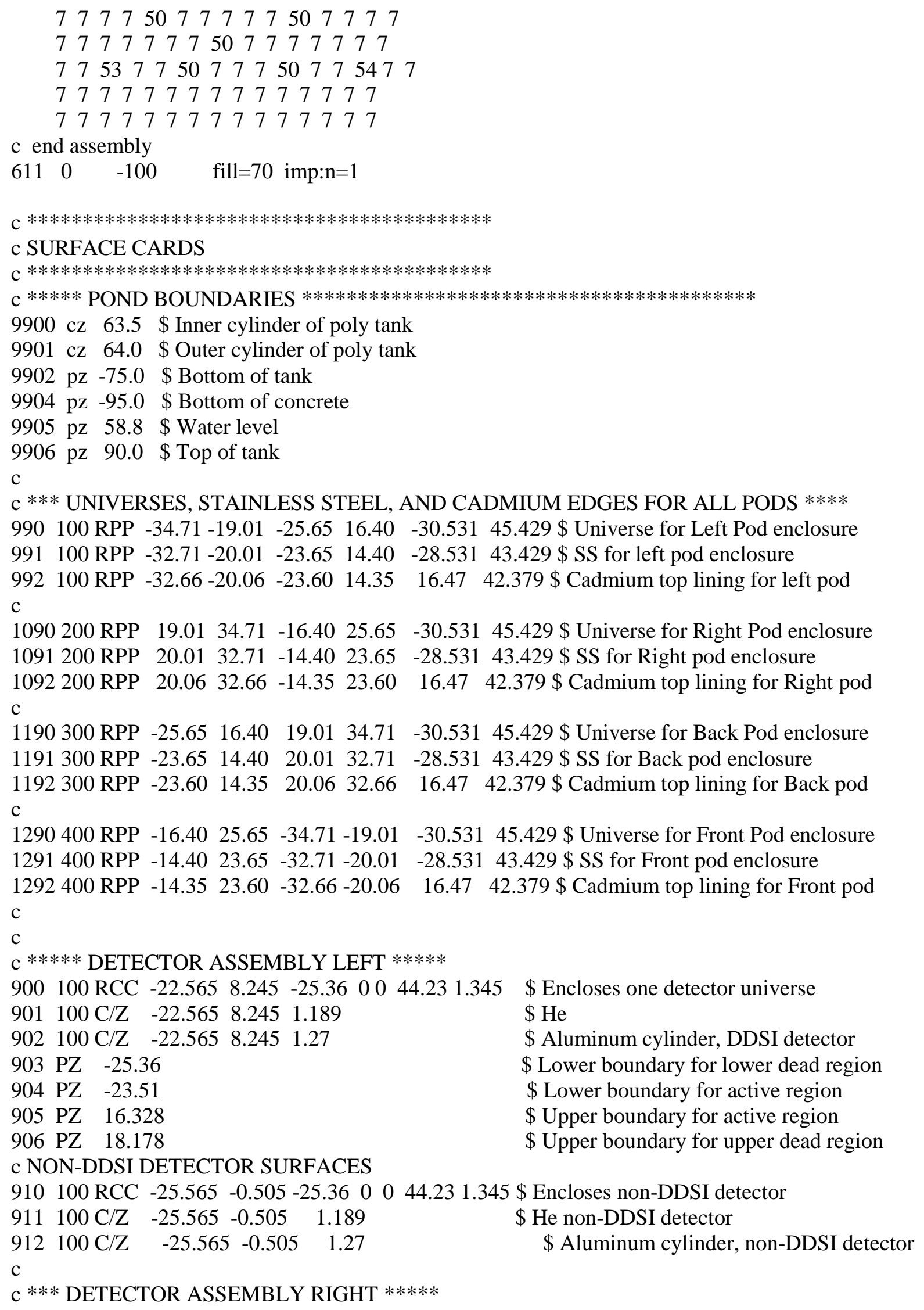




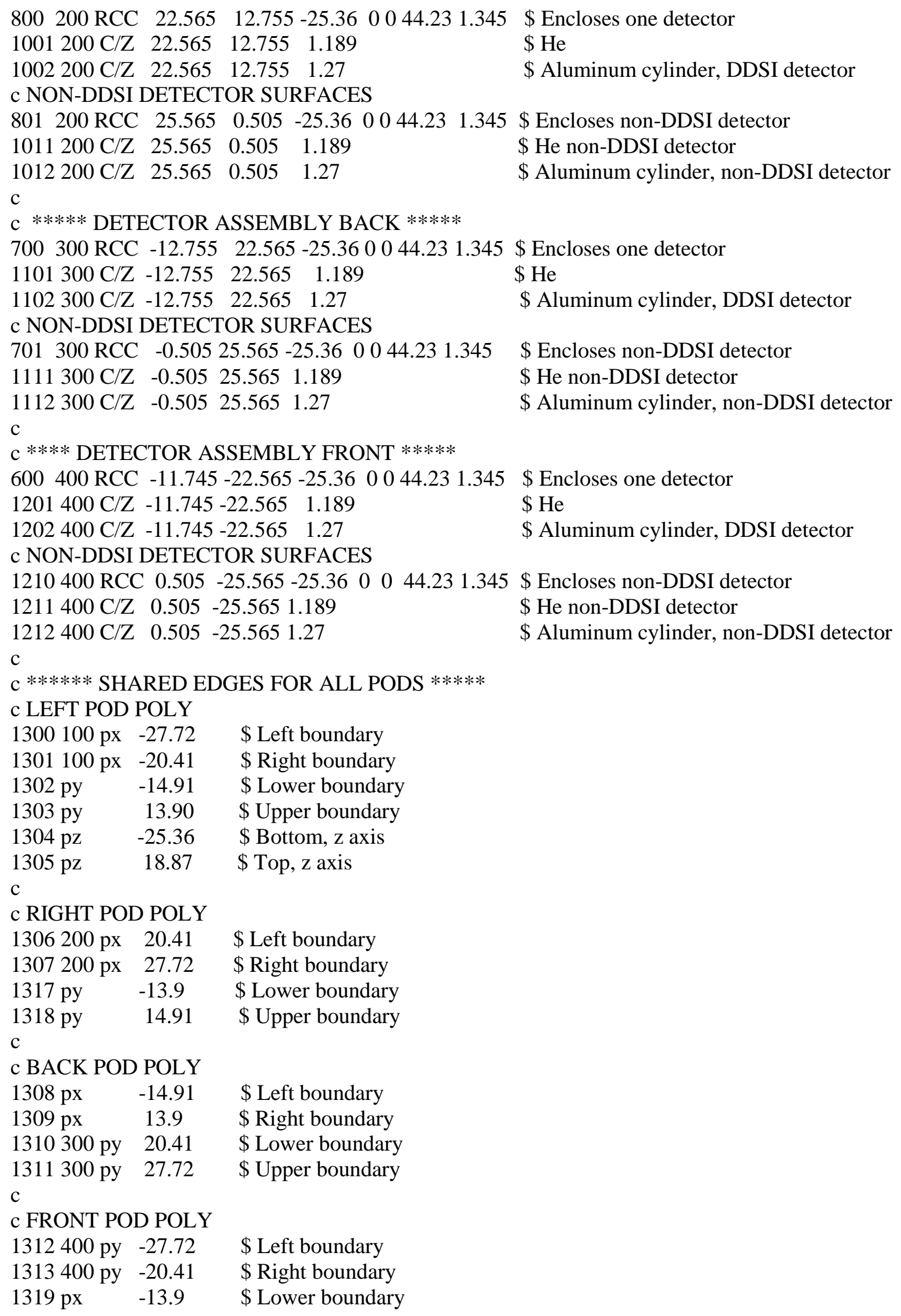




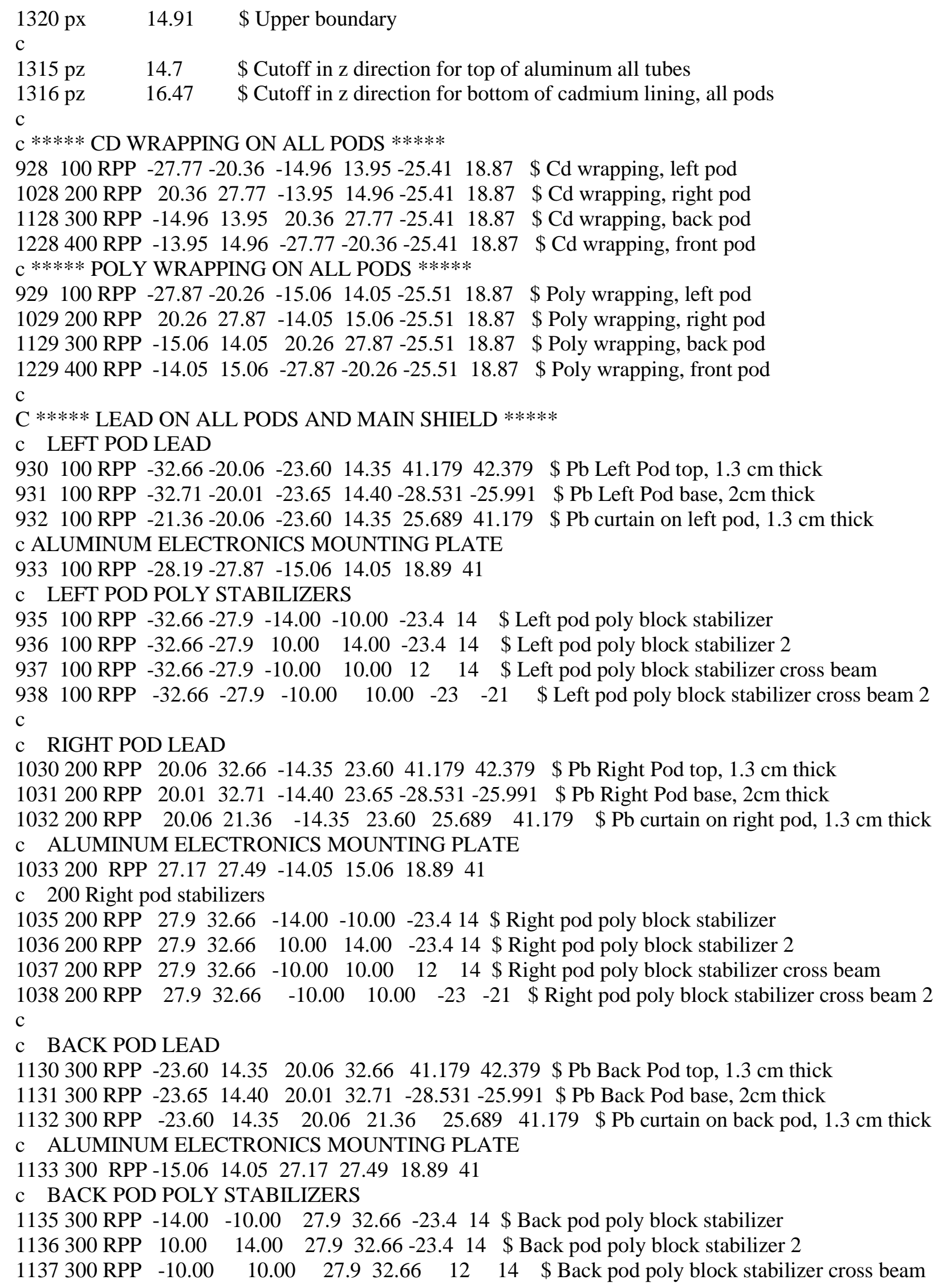




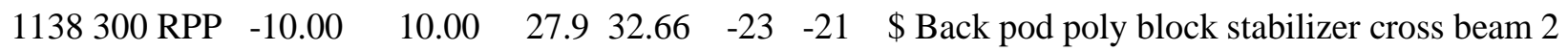
c

c FRONT POD LEAD

1230400 RPP -14.35 23.60 -32.66 -20.06 41.179 42.379 \$ Pb Front Pod top, $1.3 \mathrm{~cm}$ thick

1231400 RPP -14.40 23.65 -32.71 -20.01 -28.531 -25.991 \$ Pb Front Pod base, $2 \mathrm{~cm}$ thick

1232400 RPP $-14.35 \quad 23.60-21.36-20.06 \quad 25.689 \quad 41.179 \quad \$$ Pb curtain on front pod, $1.3 \mathrm{~cm}$ thick c ALUMINUM ELECTRONICS MOUNTING PLATE

1233400 RPP -14.05 $15.06 \quad-27.49$-27.17 18.8941

c FRONT POD POLY STABILIZERS

1235400 RPP $-14.00 \quad-10.00-32.66-27.9-23.414$ \$ Front pod poly block stabilizer

1236400 RPP $\quad 10.00 \quad 14.00 \quad-32.66-27.9 \quad-23.4 \quad 14$ \$ Front pod poly block stabilizer 2

1237400 RPP $\quad-10.00 \quad 10.00 \quad-32.66-27.9 \quad 12 \quad 14$ \$ Front pod poly block stabilizer cross beam

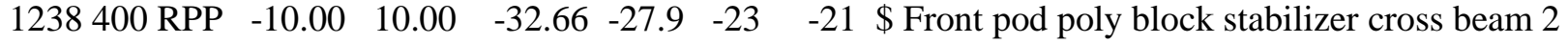

c

c MAIN LEAD SHIELD

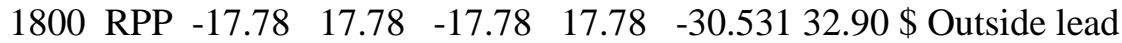

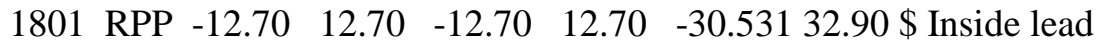

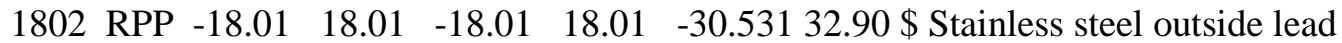

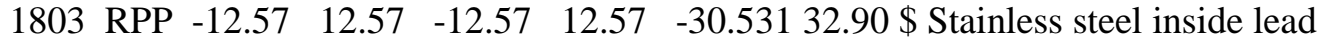

$\mathrm{c}$

c *** LIFTING MECHANISM AND FUNNEL $* * *$

1810 rpp -38.1 -37.13 -37.13 37.13 -30.531 51.04 \$ Left wall main box

1811 rpp $37.1338 .1-37.1337 .13-30.53151 .04$ \$ Right wall main box

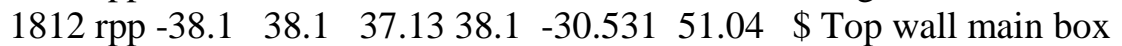

1813 rpp -38.1 $38.1 \quad-38.1-37.13-30.53151 .04 \quad$ \$ Bottom wall main box

$1814 \mathrm{rpp}-38.1 \quad 38.1 \quad-38.1 \quad 38.1 \quad-32.131-30.531$ \$ Base main box

c

C X-WISE LEFT BOXES

1815 rpp $-38.1-37.13 \quad 7.62 \quad 34.29-30.35$ 5.2 $\quad$ \$ Top, lower $x$-wise box

$1816 \mathrm{rpp}-38.1-37.13-34.29-7.62-30.355 .2 \quad$ \$ Bottom, lower x-wise box

1817 rpp $-38.1-37.13 \quad 7.62 \quad 34.29 \quad 12.8242 .0554$ \$ Top, upper x-wise box

1818 rpp -38.1 -37.13 -34.29 -7.62 12.8242 .0554 \$ Bottom, upper x-wise box

c X-WISE RIGHT BOXES

1819 rpp $37.1338 .1 \quad 7.62 \quad 34.29-30.355 .2 \quad$ \$ Top, lower $\mathrm{X}$-wise box

1820 rpp 37.1338 .1 -34.29 -7.62 -30.35 5.2 \$ Bottom, lower x-wise box

1821 rpp $37.1338 .1 \quad 7.62 \quad 34.29 \quad 12.8242 .0554$ \$ Top, upper $x$-wise box

1822 rpp 37.13 38.1 -34.29 -7.62 12.82 42.0554 \$ Bottom, upper X-wise box

c Y -WISE TOP BOXES

1823 rpp -34.29 -7.62 37.13 38.1 -30.35 5.2 \$ Top, lower y-wise box

$1824 \mathrm{rpp} 7.62 \quad 34.2937 .1338 .1$-30.35 5.2 \$ Bottom, lower y-wise box

1825 rpp -34.29 $-7.62 \quad 37.1338 .1 \quad 12.8242 .0554$ \$ Top, upper y-wise box

1826 rpp $7.6234 .2937 .1338 .1 \quad 12.8242 .0554 \quad$ \$ Bottom, upper y-wise box

c $\mathrm{y}$-wise bottom boxes

1827 rpp -34.29 -7.62 -38.1 -37.13 -30.35 5.2 \$ Top, lower y-wise box

1828 rpp $7.62 \quad 34.29-38.1-37.13-30.355 .2 \quad$ \$ Bottom, lower y-wise box

1829 rpp -34.29 -7.62 -38.1 -37.13 12.8242 .0554 \$ Top, upper y-wise box

1830 rpp $7.62 \quad 34.29-38.1-37.13 \quad 12.8242 .0554$ \$ Bottom, upper y-wise box

c

c FUNNEL

$1831 \quad$ rpp $-11.1 \quad 11.1 \quad-11.1 \quad 11.1 \quad-27.7652 .38 \quad$ \$ Funnel inside

$1832 \quad$ rpp $-11.33 \quad 11.33 \quad-11.33 \quad 11.33-27.7652 .38$ \$ Funnel outside 
183330 rpp $-47.7547 .75-2.91852 .918551 .152 .38$ \$ Funnel support 1 183430 rpp $-2.91852 .9185-47.7547 .7551 .152 .38$ \$ Funnel support 2

$\mathrm{c}$

c LIFTING SUPPORTS ON TOP OF BOX

$720 \quad$ rpp -2.3 $2.3 \quad-38.1-28.932652 .9487 .14$ \$ Outside base lift bottom

$721 \quad$ rpp -1.7 $1.7 \quad-37.5-29.532653 .5486 .54 \quad$ \$ Inside base lift

$722 \quad$ rpp -38.1 -28.9326-2.3 $2.3 \quad 52.9487 .14 \quad$ \$ Outside base lift left

$723 \quad$ rpp -37.5 -29.5326-1.7 $1.7 \quad 53.5486 .54 \quad$ \$ Inside base lift

$72431 \mathrm{rpp}-2.3 \quad 2.3 \quad-38.1-28.932652 .9487 .14 \quad$ \$ Outside base lift bottom left

$72531 \mathrm{rpp}-1.7 \quad 1.7 \quad-37.5-29.532653 .5486 .54 \quad$ \$ Inside base lift

$\mathrm{c}$

c *** CF SPHERES AND SOURCE RODS ***

$\mathrm{c}$

101 SPH 00 -5.335 $0.0002 \quad$ \$ Sphere for Cf-252

106 RCC $00-5.035 \quad 0063.840 .2 \quad$ \$ Rod

c

102 SPH $00-14.12 \quad 0.0002 \quad$ \$ Sphere for Cf-252

107 RCC $00-13.820072 .620 .2 \quad$ \$ Rod

c

103 SPH $00 \quad 6.47 \quad 0.0002 \quad$ \$ Sphere for Cf-252

108 RCC $00 \quad 6.77 \quad 0052.030 .2 \quad$ \$ Rod

c

104 SPH 00 -25.28 $0.0002 \quad$ \$ Sphere for Cf-252

109 RCC $00-24.98 \quad 0083.780 .2 \quad$ \$ Rod

c

105 SPH $00 \quad 15.8 \quad 0.0002 \quad$ \$ Sphere for Cf-252

110 RCC $00 \quad 16.1 \quad 0042.700 .2 \quad$ \$ Rod

$\mathrm{C}$

c $* * *$ LEU FUEL RODS $* * *$

$601 \mathrm{c} / \mathrm{z} 000.462 \quad$ \$ Fuel

$602 \mathrm{c} / \mathrm{z} 000.537$ \$ Outer clad

c *** SUS 304 GUIDE TUBES $* * *$

$604 \mathrm{C} / Z 000.571 \quad$ \$ Guide tube inner diameter

$605 \mathrm{C} / \mathrm{Z} 000.613$ \$ Guide tube outer diameter

$\mathrm{C} * * *$ GD FUEL RODS $* * *$

$606 \mathrm{c} / \mathrm{z} 000.4515 \quad$ \$ Active Length 122d5

607 c/z 000.5385 \$ Outside Diameter Rod

$\mathrm{c} * * *$ DU FUEL RODS $* * *$

$608 \mathrm{c} / \mathrm{z} 000.4515$ \$FUEL

$609 \mathrm{c} / \mathrm{z} 000.5385$ \$OUTER CLAD

c

c *** SUPPORT STRUCTURES, HORIZONTAL SS $* * *$

$610 \mathrm{pz} 14.32$ \$ Upper thin hor. support (bottom) verified, 7/20/15

$611 \mathrm{pz} 15.32$ \$ Upper thin hor. support (top)verified, 7/20/15

$612 \mathrm{pz}-18.7$ \$ Lower thin hor. support (bottom) verified, 7/20/15

$613 \mathrm{pz}-17.7$ \$ Lower thin hor. support (top)verified, 7/20/15

$614 \mathrm{pz} 57.8$ \$ Upper spacer bottom

$615 \mathrm{pz} 59.8$ \$ Upper spacer top

c

c *** DEAD REGION AND WATER LEVEL Z PLANES ***

$620 \mathrm{pz}-47.61$ \$ Start of LEU fuel region 
621 pz 54.39 \$ Dead region top LEU fuel

$622 \mathrm{pz}-57.11$ \$ Dead region DU fuel

$623 \mathrm{pz} 63.89$ \$ Dead region top DU fuel

$624 \mathrm{pz} 58.8$ \$ Water level

c

c *** ASSEMBLY LATTICE ***

99 rpp $-0.7215 \quad 0.7215 \quad-0.7215 \quad 0.7215 \quad-61 \quad 67$

100 rpp -10.75 $10.75 \quad-10.75 \quad 10.75 \quad-59.61 \quad 66.39 \quad$ \$ Verified, 7/20/15

$\mathrm{c} * * * * * * * * * * * * * * * * * * * * * * * * * * * * * * * * * * * * * * * * * *$

c DATA CARDS

$\mathrm{c} * * * * * * * * * * * * * * * * * * * * * * * * * * * * * * * * * * * * * * * * * * * *$

fmult 94236 WIDTH $=1.1$ WATT $=.24$ SFYIELD 5.57E +04

fmult 96246 WIDTH $=1.1$ WATT $=.24$ SFYIELD 8.77E +06

fmult 96248 WIDTH $=1.1$ WATT $=.24$ SFYIELD 4.12E +07

fmult $98250 \mathrm{WIDTH}=1.1 \mathrm{WATT}=.24$ SFYIELD $1.09 \mathrm{E}+10$

$\mathrm{c}$

c TRANSLATIONS

TR100 0.500

TR200 -0.500

TR300 $0-0.50$

TR400 00.50

TR30* $00045-45901354590909001$

TR31*0 $0045-45901354590909001$

C

$\mathrm{c} * * * * * * * * * * * * * * * * * * * * *$

c TALLIES

$\mathrm{c} * * * * * * * * * * * * * * * * * * * * *$

fc8 He3 Captures - No Gate

f8:n (900 100011001200$)$

$\mathrm{ft} 8$ CAP 2003

c

fc18 Coincidence - PD 0 GW 2

f18:n (900 10001100 1200)

ft18 CAP 2003 GATE 0200

$\mathrm{c}$

fc28 Coincidence - PD 2 GW 2

f28:n (900 10001100 1200)

ft28 CAP 2003 GATE 200200

c

fc38 Coincidence - PD 4 GW 2

f38:n (900 10001100 1200)

ft38 CAP 2003 GATE 400200

c

$\cdots$

c

fc998 Coincidence - PD 196 GW 2

f998:n (900 10001100 1200)

ft998 CAP 2003 GATE 19600200

c

c DDSI TALLIES END 


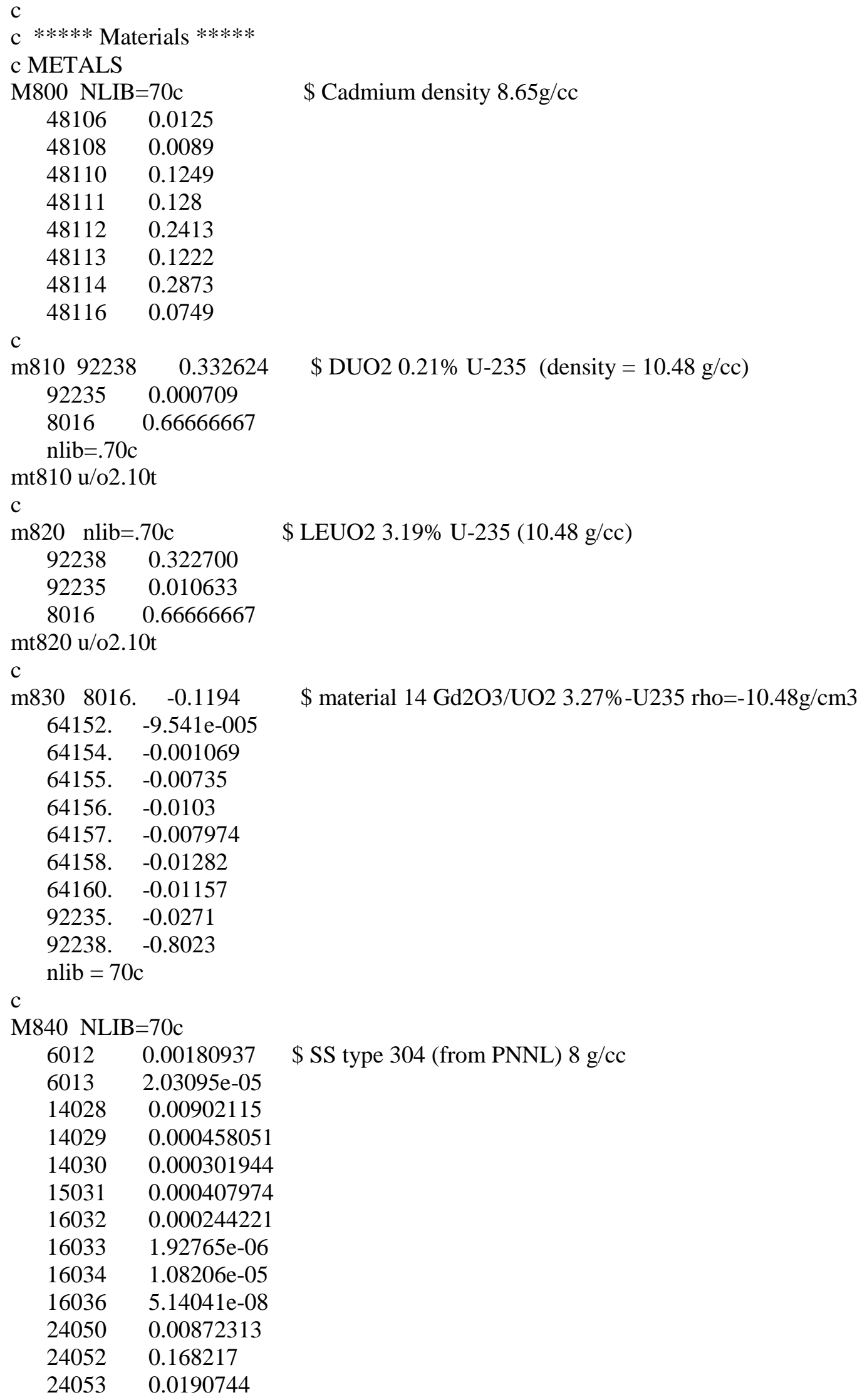

\$ LEUO2 3.19\% U-235 (10.48 g/cc)

\$ material 14 Gd2O3/UO2 3.27\%-U235 rho=-10.48g/cm3 


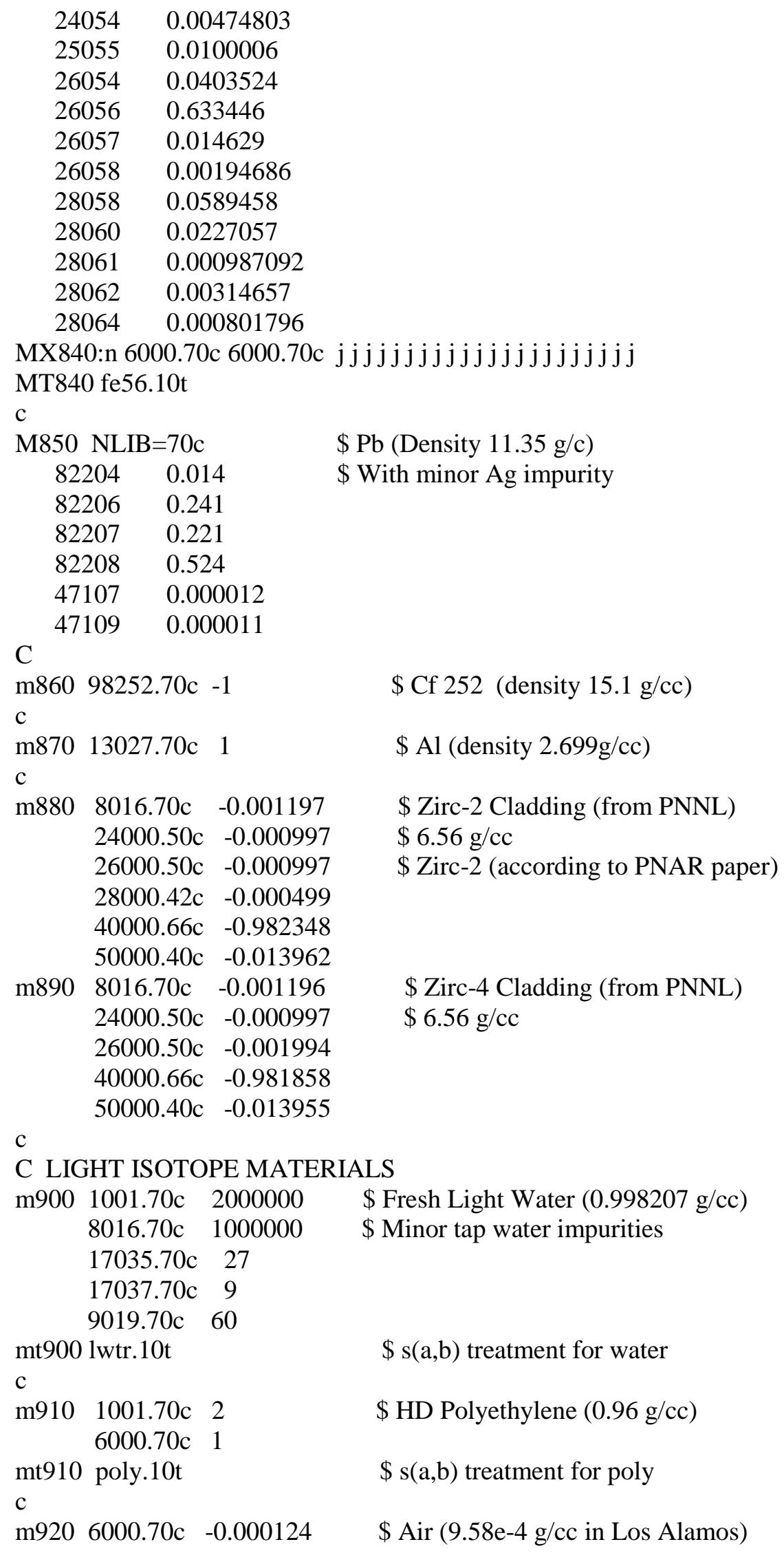




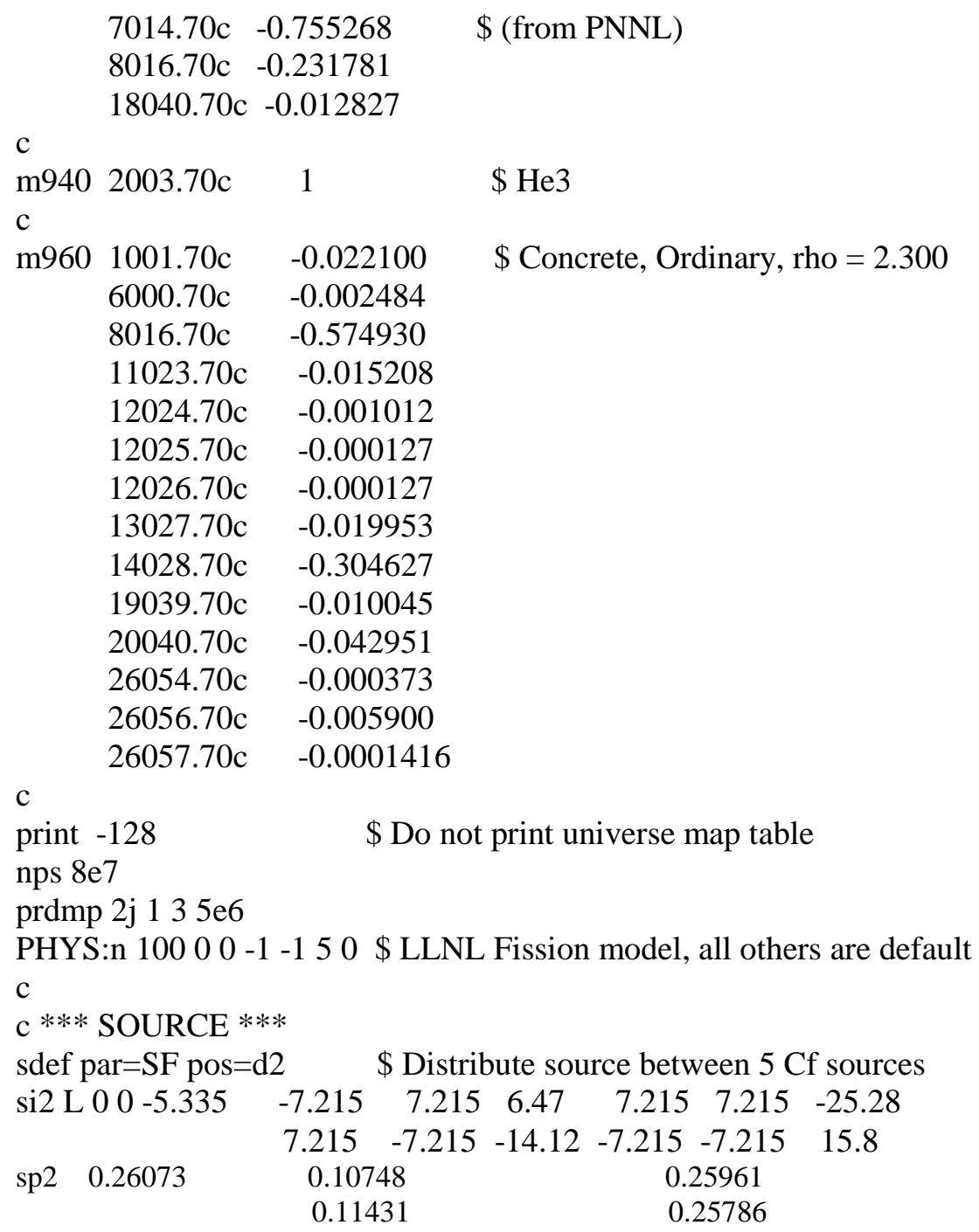




\section{Appendix D. Example Python Analysis Script}

\#!/usr/bin/python

\# Post-processing and RAD analysis of MCNP spent fuel tally data

import os

import numpy as $\mathrm{np}$

from scipy.optimize import curve_fit

import matplotlib.pyplot as plt

from itertools import islice

from pylab import * \# for array, zeros, arange

\# This function takes values to make an exponential and returns

\# the calculated values

def func(x, Tau, A):

return $A * n p . \exp (-1 * x /$ Tau $)$

\# This function finds the index of the first number in a set of 3 that \# is below a maxVal/divisor. This should find the place where the \# entire curve has fallen low enough, ignoring noise def lowVal(a,maxVal,divisor):

consCntr $=0$

$\mathrm{i}=0$

while consCntr $<3$ :

if a[i] $<\mathrm{maxVal} /$ divisor:

consCntr $=$ consCntr +1

elif a[i] > maxVal/divisor:

consCntr $=0$

$\mathrm{i}=\mathrm{i}+1$

firstSet $=\mathrm{i}-3$

return firstSet

def general_fit(f, xdata, ydata, $\mathrm{p} 0=$ None, sigma=None, $\left.{ }^{* *} \mathrm{kw}\right)$ :

Pass all arguments to curve_fit, which uses non-linear least squares

to fit a function, $\mathrm{f}$, to data. Calculate the uncertaities in the

fit parameters from the covariance matrix.

"'"'

popt, pcov $=$ curve_fit $(f$, xdata, ydata, p0, sigma, $* *$ kw $)$ 


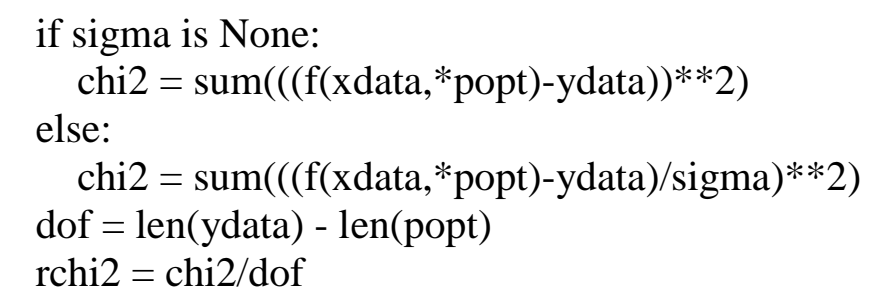

\# The uncertainties are the square roots of the diagonal elements punc $=\operatorname{zeros}($ len $($ popt $))$

for $i$ in arange(0,len(popt)): $\operatorname{punc}[\mathrm{i}]=\operatorname{sqrt}(\operatorname{pcov}[\mathrm{i}, \mathrm{i}])$

return popt, punc, rchi2, dof

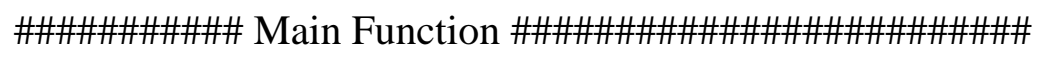
def main():

\# Define path from which output files will be obtained

full_path = os.path.realpath (__file__ $)$

my_path = os.path.dirname $($ full_path $)+$ '/'

\# Change this to describe the output currently being processed output_desc = '_lift_t'

\#Open output file, write 'header' values into file out = open (my_path +'All_outputs' + output_desc + '.txt','w') out.write(output_desc + 'In')

out.write("BU IE CT Mult Fast_A Fast_Tau Slow_A Slow_Tau " "Early_Tau E_Tau_Err Fast_Area Slow_Area Singles" + "In")

\# Define bin times in RAD

usTimes $=$ range $(0,197,2)$

\# Open output file for all outputs and input file, which is a condensed \# tally data file outFile $=$ open $($ my_path + 'Detailed_Output' + output_desc + '.txt',"w") inputFile $=$ my_path + "Tally_Data" + output_desc + ".txt"

\# Try to open the input file, if not able, exit loop gracefully try:

infile $=$ open(inputFile, ' $r$ ')

except IOError:

print('There was an error opening the tally data input file')

for line in infile:

tokens $=$ line.split ()

if tokens[0] == 'Total': 
numFiles $=\operatorname{int}($ tokens[1])

infile.close()

\# Reopen the input file, if not able, exit loop gracefully

try:

infile $=$ open $\left(\right.$ inputFile, ${ }^{\prime} r$ ')

except IOError:

print('There was an error opening the input file')

\# Set parameters for slow fit

startSlow $=48 \#$ Start at time 97 us $(48$ th index, $=2 * 48+1)$ for calculating

\# slow component

endSlow $=97$ \# End at time 194 us (97th index)

earlyEnd $=26$ \# Early end at 26th index, $=2 * 25+1$

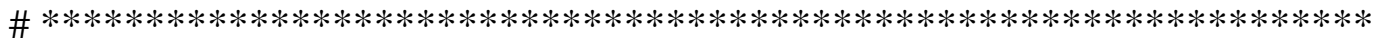

\# Go through each file in condensed tally input file, pull out pertinent

\# information

for files in range(numFiles):

headerData $=$ list $($ islice $($ infile, 4$))$ \# Slice header data

curFile = headerData[0].split() \# Split first line for curFile

mult =headerData[1].split() \# Split second line for mult

singRates $=$ float (headerData[2]) \# Singles on third line, float

dataLength $=\operatorname{int}($ headerData[3]) $\quad$ \# Data length on fourth line, int

\# Get BU, IE, CT from curFile from header

$\mathrm{BU}=$ curFile[0]

$\mathrm{IE}=$ curFile[1]

$\mathrm{CT}=$ curFile[2]

\# Using data length, pull out correct number of lines with doubles info

dubs =list(islice(infile, dataLength))

dubRates $=[]$

dubErrs $=[]$

for items in dubs:

itemSplit = items.split()

dubRates.append(float(itemSplit[0])) \# Get doubles rates

dubErrs.append(float(itemSplit[1])) \# Get doubles errors

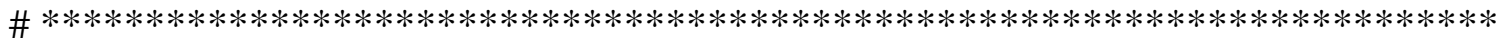

\# Compute slow fit, then find vals to be subtracted for fast fit

xdataS $=$ np.array (usTimes[startSlow:endSlow])

ydataS $=$ np.array $($ dubRates [startSlow:endSlow $])$

pS0 $=$ np.array $([100$, dubRates[0]/2] $) \#$ Beginning guess for slow magnitude

\# is first dubRates val / 2 
\# Check for a file with no doubles information, print error if needed if len $($ ydataS $)==0$ :

print ("Error! "+ str(BU) + "GWd_" + str(IE) + "p_"+ str(CT) + "y contains no doubles information")

continue

\# Slow curve fitting process using curve_fit, then compute slow

\# function values and area

poptS, pcovS = curve_fit(func, $x$ dataS, ydataS,pS0)

slow Tau $=$ poptS[0]

slow $\mathrm{A}=\operatorname{poptS}[1]$

slowFuncVals = func(np.array(usTimes),slowTau,slowA)

slowArea $=\operatorname{sum}($ slowFuncVals $)$

\# Find index of first value in set of 3 below 0

maxFast $=$ fastVals.index $(\max ($ fastVals $))$ \# Find index of maximum fast value

endFast $=$ lowVal(np.array(fastVals),fastVals[maxFast],1000)

\# Compute fast fit based on vals after subtraction, only up to endFast

xdata $=$ np.array(usTimes[0:endFast $]$ )

ydata $=$ np.array $($ fastVals $[0:$ endFast $])$

$\mathrm{pF} 0=\mathrm{np} \cdot \operatorname{array}\left(\left[18, \operatorname{dubRates}[0]^{*} 0.75\right]\right)$

\# Perform least squares curve fitting to fast data, then

\# compute fast values and area

popt, pcov $=$ curve_fit(func, $x$ data, $y d a t a, p F 0)$

fastTau $=$ popt $[0]$

fast $\mathrm{A}=\operatorname{popt}[1]$

fastFuncVals = func(np.array(usTimes),fastTau,fastA)

\# Compute early fit; start at time $=6$ us

xEarlyData $=$ np.array $($ usTimes[3:earlyEnd] $)$

yEarlyData $=$ np.array $($ dubRates $[3:$ earlyEnd $])$

\# Use general fit function to get early tau and magnitude, plus errors

poptE,puncE,rchi2E,dofE = general_fit(func,xEarlyData,

earlyTau $=$ poptE[0]

yEarlyData,pF0,dubErrs[1:earlyEnd])

earlyA = poptE[1]

earlyFitVals = func (xEarlyData, earlyTau, earlyA)

fastArea $=\operatorname{sum}($ dubRates $[2: 10])$

\# $* * * * * * * * * * * * * * * * * * * * * * * * * * * * * * * * * * * * * * * * * * * * * * * * * * * * * * * * * * * * * * * * * * * * * * *$

\# Write the pertinent information to the master output file

out.write(BU + " " + IE + " " + CT + " ") 
out.write( $\operatorname{str}\left(' \% .4 \mathrm{f}^{\prime} \%\right.$ (float(mult[0] $\left.\left.)\right)\right)+"$ " $+\operatorname{str}\left(' \% .4 \mathrm{f}^{\prime} \%\right.$ fastA $)+$

" " +str('\%.4f' \% fastTau $)+"$ " +str('\%.4f' \% slowA $)+"$ " +

$\operatorname{str}\left(' \% .4 f^{\prime} \%\right.$ slowTau $)+" ~ "+\operatorname{str}\left(' \% .4 f^{\prime} \%\right.$ earlyTau $)+" ~ "+$

$\operatorname{str}\left(' \% .4 \mathrm{f}^{\prime} \%\right.$ puncE[0]) + ' ' $+\operatorname{str}\left(' \% .4 \mathrm{f}^{\prime} \%\right.$ fastArea $)+"$ " +

str('\%.4f' \% slowArea) + " " + str('\%.4f' \% singRates) +"'n")

\# Write the pertinent detailed information to a file

outFile.write(BU + " " + IE + " " + CT + " " + str('\%.4f' \% (float(mult[0]))) +

$$
\text { ' ' }+\operatorname{str}\left(' \% .4 \mathrm{f}^{\prime} \% \text { singRates }\right)+\text { ' ') }
$$

outFile.write(' '.join(map(str,dubRates)) + "In")

\# $* * * * * * * * * * * * * * * * * * * * * * * * * * * * * * * * * * * * * * * * * * * * * * * * * * * * * * * * * * * * * * * * * * * * * * * *$

\# Plot the figure for each case, save it, clear it from memory

fig = plt.figure $(1)$

font $=\{$ 'family' : 'Times New Roman',

'serif' : 'Times New Roman',

'size' : 18$\}$

plt.rc('font', **font)

plt.subplots_adjust $($ left $=0.15)$ \# Leave margin on the left

plt.plot(usTimes, dubRates, 'ro',label='RAD') \# Plot RAD

plt.plot(usTimes, slowFuncVals, 'b-.',linewidth = 3.0, label='Slow Component, \$ltau\$ = ' + str('\%i' \% round(slowTau)) + ' ' + r'\$ $\$$ mu\$' + 's') \# Slow curve

plt.plot(usTimes,fastFuncVals, 'g--',linewidth $=3.0$, label='Fast Component, \$1tau\$ = '+ str('\%i' \% round(fastTau)) + ' ' + r'\$ $\$$ mu \$' + 's') \# Fast curve

plt.plot(xEarlyData,earlyFitVals,'gs',label='Early Fit, \$lltau\$ = ' + str('\%i' \% round(earlyTau)) + ' ' + r'\$ $\backslash$ mu $\$$ ' + 's',

markersize = 7) \# Early curve

plt.legend $(\operatorname{loc}=1$, borderpad $=.1$, labelspacing $=.1$, numpoints $=3$ )

plt.xlabel('Time after Trigger [' + r'\$lmu\$' + 's]')

plt.ylabel("Doubles Rate [1/s]")

plt.title(BU + "GWd/tU, " + IE + "\%, " + CT + "y")

fig.savefig(my_path + "Images2/TNR" + BU + " GWd_" + IE + "p_" + CT +

plt.clf()

"y" + output_desc + ".png")

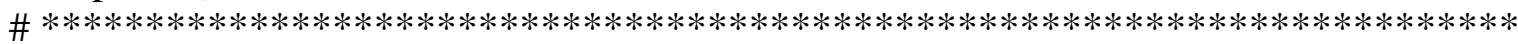

\#Plot the raw figure for each case, save it, clear it from memory

fig $=$ plt.figure $(1)$

font $=\{$ 'family' : 'Times New Roman',

'serif' : 'Times New Roman',

'size' : 22\}

plt.rc('font', **font)

plt.subplots_adjust $($ left $=0.15)$

plt.subplots_adjust $($ bottom $=0.15)$ 


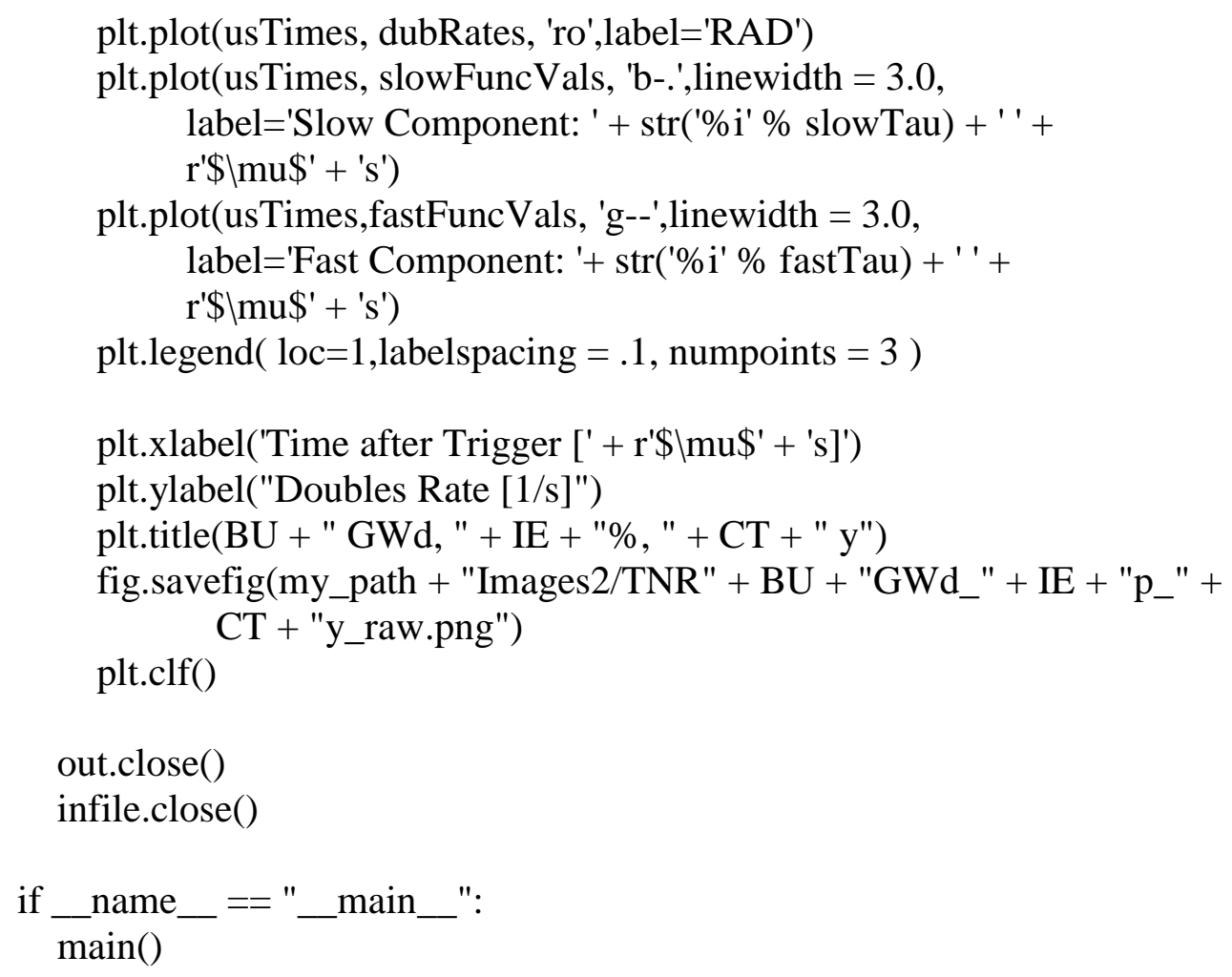




\section{REFERENCES}

${ }^{1}$ T. D. Reilly, N. Ensslin, H. A. Smith, S. Kreiher (Eds.), Passive Nondestructive Assay of Nuclear Materials, NUREG/CR-5550 (1991).

2 A. Scheinman, "Calling for Action: The Next Generation Safeguards Initiative," The Nonproliferation Review, Vol. 16, No. 2, July (2009).

${ }^{3}$ H. O. Menlove, S. H. Menlove, S. J. Tobin, "Fissile and fertile nuclear material measurements using a new differential die-away self-interrogation technique", Nuclear Instruments and Methods in Physics Research A, Vol. 602, pp 588-593, (2009).

${ }^{4}$ H. O. Menlove, S. H. Menlove, and S. J. Tobin, "Verification of Plutonium Content in Spent Fuel Assemblies Using Neutron Self-Interrogation," Proceedings of the $50^{\text {th }}$ Annual Meeting of the Institute of Nuclear Materials Management, Tuscon, AZ July 12-16, (2009).

${ }^{5}$ ASTM C1493-09, Standard Test Method for Non-Destructive Assay of Nuclear Material in Waste by Passive and Active Neutron Counting Using a Differential Die-Away System," ASTM International, West Conshohocken, PA, (2009).

${ }^{6}$ D. G. Langer, M. S. Krick, N. Ensslin, G. E. Bosler, and N. Dytlewski, "Neutron Multiplicity Counter Development," ESARDA proceedings, Avignon, France. May 14-16, (1991).

${ }^{7}$ M. A. Schear, H. O. Menlove, S. J. Tobin, L. G. Evans, S. Y. Lee, "Fissile Material Measurements using the Differential Die-Away Self-Interrogation Technique," Proceedings of the $51^{\text {st }}$ Annual Meeting of the Institute of Nuclear Materials Management, Baltimore, MD July 11-15, (2010).

${ }^{8}$ A. C. Kaplan, V. Henzl, A. P. Belian, H. O. Menlove, M. T. Swinhoe, S. J. Tobin, M. Flaska, S. A. Pozzi, "Simulation of Spent Fuel Isotopic Content Measurement with the Differential Die-Away Self-Interrogation System", Proceedings of the 54th Annual Meeting of the Institute of Nuclear Materials Management, Palm Desert, CA July 14-18. (2013)

${ }^{9}$ A. C. Kaplan, V. Henzl, A. P. Belian, H. O. Menlove, M. T. Swinhoe, M. Flaska, S. A. Pozzi, "Determination of Spent Nuclear Fuel Assembly Multiplication with the Differential Die-Away SelfInterrogation Instrument," Nuclear Instruments and Methods A, Vol. 757, No. 1, (2014).

${ }^{10}$ M. A. Schear, H. O. Menlove, L. G. Evans, S. J. Tobin, and S. Croft, "Development of the Differential Die-Away Self-Interrogation Technique for Spent Fuel Characterization," US Department of Energy report, (2010). 
${ }^{11}$ A. P. Belian, H.O. Menlove, M. T. Swinhoe, and S.J. Tobin. New Design of the Differential DieAway Self-Interrogation Instrument, Journal of Nuclear Materials Management, Vol. 40, No. 3, pp 58-60, (2012).

${ }^{12}$ D. Henzlova, H. O. Menlove, M. T. Swinhoe, J. B. Marlow, I. P. Martinez, C. D. Rael, "Neutron Data Collection and Analysis Techniques Comparison for Safeguards", IAEA-CN-184/178.

${ }^{13}$ M. A. Humphrey, S. J. Tobin, K. D. Veal, “The Next Generation Safeguards Initiative's Spent Fuel Nondestructive Assay Project," Journal of Nuclear Materials Management, Vol. 40, No. 3, pp $1-6,(2012)$.

${ }^{14}$ P. M. Rinard and G. E. Bosler, "Safeguarding LWR Spent Fuel with the Fork Detector," Los Alamos National Laboratory Report (LA-11096-MS), (1988).

${ }^{15}$ A. Tiitta, J. Saarinen, M. Tarvainen, K. Axell, P. Jansson, R. Carchon, J. Gerits, Y. Kulikov, and Y. G. Lee, "Investigation on the Possibility to use Fork Detector for Partial Defect Verification of Spent LWR Fuel Assemblies,” Report to IAEA Safeguards, STUK, September (2002).

${ }^{16}$ K. Van der Meer, M. Coeck, "Is the fork detector a partial defect tester?" IAEA-CN-148/68, IAEA Symposium on Addressing Verification Challenges, Vienna, Austria, October 16-20, (2006).

${ }^{17}$ B. Richter, C. P Behrens, H. H. Remagen, A. Rezniczek, K. Rudolf, G. Stein, M. Weis, "Safeguards for spent nuclear fuel in transfer from wet storage to dry storage in on-site interim storage facilities," IAEA-CN-148/114, IAEA Symposium on Addressing Verification Challenges, Vienna, Austria, October 16-20, (2006).

${ }^{18}$ J. D. Chen et. al., "Digital Cerenkov Viewing Device for Verification of Spent LWR Fuel," IAEA, (1997).

${ }^{19}$ A. Lebrun et al., "SMOPY a new NDA tool for safeguards of LEU and MOX spent fuel," International Atomic Energy Agency Report IAEA-SM-367/14/03, 47, (2003).

${ }^{20}$ L. G. Evans, M. A. Schear, S. Croft, S. J. Tobin, M. T. Swinhoe, and H. O. Menlove, "Nondestructive Assay of Spent Nuclear Fuel using Passive Neutron Albedo Reactivity," Proceedings of the 51st Annual Meeting of the Institute of Nuclear Materials Management, Baltimore, MD, July 11-15, (2010).

${ }^{21}$ W. S. Charlton, D. Strohmeyer, A. Stafford, S. Saavedra, and A. S. Hoover, "The Use of SelfInduced XRF to Quantify the Pu Content in PWR Spent Nuclear Fuel," European Safeguards R\&D Association, Vilnius, Lithuania, (2009).

${ }^{22}$ D. K. Min, H. J. Park, K. J. Park, D. G. Park, S. G. Ro and H. S. Park, "Determination of Burnup, Cooling time and Initial Enrichment of PWR spent fuel by use of Gamma-ray activity ratios," IAEA, Taejon, Korea, (1998).

${ }^{23}$ T. Lee, H. O. Menlove, S. Lee, H. Kim, "Development of the ACP safeguards neutron counter for PWR spent fuel rods," NIM A, Vol. 589, pp 57-65, (2008). 
${ }^{24}$ V. Henzl, M. T. Swinhoe, S. J. Tobin, and H. O. Menlove, "Measurement of the Multiplication of a Spent Fuel Assembly with the Differential Die-Away Method within the Scope of the Next Generation Safeguards Initiative Spent Fuel Project." JNMM-Journal of the Institute of Nuclear Materials Management 40.3 (2012).

${ }^{25}$ P. Blanc, S. J. Tobin, T. Lee, J. Hu, J. Hendricks, and S. Croft, " Delayed Neutron Detection with an Integrated Differential Die-Away and a Delayed Neutron Instrument," Proceedings of the $51^{\text {st }}$ INMM Conference, Baltimore, MD, July (2010).

${ }^{26}$ V.V. Mozin, SJ. Tobin, J. Vujic, B. Ludewigt, A.W. Hunt, "Delayed Gamma Technique for Fissile Material Assay," Institute of Nuclear Materials Management 51st Annual Meeting, Baltimore, MD, July 11-16, (2010).

${ }^{27}$ J. Hu, S. J. Tobin, H. O. Menlove, M. T. Swinhoe, and S. Croft, "Developing the Californium Interrogation Prompt Neutron Technique to Measure Fissile Content and to Detect Diversion in Spent Nuclear Fuel Assemblies", Journal of Nuclear Materials Management, Vol. 40, No. 3, pp 3644, (2012).

${ }^{28}$ T. W. Crane, "Measurement of Uranium and Plutonium in Solid Waste by Passive Photon or Neutron Counting and Isotopic Neutron Source Interrogation," Informal LANL report, LA-8294MS, (1980).

${ }^{29}$ A. M. LaFleur, W. S. Charlton, H. O. Menlove, M. T. Swinhoe, "Comparison of fresh fuel experimental measurements to MCNPX calculations using self-interrogation neutron resonance densitometry", Nuclear Instruments and Methods in Physics Research A, Vol. 680, pp 168-178, (2012).

${ }^{30}$ L. E. Smith, K. K. Anderson, J. J. Ressler, M. W. Shaver, "Time-Spectral Analysis Methods for Spent Fuel Assay Using Lead Slowing-Down Spectroscopy," IEEE Transactions on Nuclear Science, Vol. 57, Issue 1, (2010).

${ }^{31}$ J. W. Behrens, R. G. Johnson, R. A. Schrack, "Neutron Resonance Transmission Analysis of Reactor Fuel Samples," ANS Nuclear Technology, Vol. 67, No. 1, pp 162-168, (1984).

${ }^{32}$ B. Quiter, B. A. Ludewigt, V. V. Mozin, and S. J. Tobin, "Nondestructive Spent Fuel Assay Using Nuclear Resonance Fluorescence," Institute of Nuclear Materials Management 50th Annual Meeting, Tucson, AZ, July 12-16, (2009).

${ }^{33}$ V. Henzl, S. Croft, M. T. Swinhoe, S. J. Tobin, "Determination of the plutonium content in a spent fuel assembly by passive and active interrogation using a differential die-away instrument," Nuclear Instruments and Methods in Physics Research A, Vol. 712 (2013).

${ }^{34}$ A. C. Kaplan, V. Henzl, H. O. Menlove, M. T. Swinhoe, A. P. Belian, M. Flaska, S. A. Pozzi, "Determination of Total Elemental Plutonium Mass with the Differential Die-Away SelfInterrogation Instrument,"NIM A, Vol. 764, No. 1, pp 347-351, August (2014).

${ }^{35} \mathrm{~K}$. Boehnel, "Determination of Plutonium in Nuclear Fuels Using the Neutron Coincidence Method," KFK2203, Karlsruhe, 1975, and AWRE Translation 70 (54/4242), Aldermaston, (1978). 
${ }^{36}$ S. Croft, A. Favalli, D. K. Hauck, D. Henzlova, P. A. Santi, "Feynman variance-to-mean in the context of passive neutron coincidence counting," Nuclear Instruments and Methods A, Vol. 686, pp 136-144, (2012).

${ }^{37}$ Korea Atomic Energy Research Institute, Nuclear Data Center, http://atom.kaeri.re.kr/.

${ }^{38}$ K. A. Jordan, J. Vujic, T. Gozani, "Remote thermal neutron die-away measurements to improve Differential Die-Away Analysis," Nuclear Instruments and Methods A, Vol. 579, No. 1, pp 407-409, (2007).

${ }^{39}$ M. Bruggeman, P. Baeten, W. De Boeck, R. Carchon, "Neutron coincidence counting based on time interval analysis with one- and two-dimensional Rossi-alpha distributions," Nuclear Instruments and Methods in Physics Research A, Vol. 382, pg. 511-518, (1996).

${ }^{40}$ E. Sakai et. al., "Temperature Dependence of Thermal Neutron Detection Performance of BF3 Proportional Counters," IEEE Transactions on Nuclear Science, Vol. 30, Issue 1, pp 802-805, (1980).

${ }^{41}$ G. F. Knoll Radiation Detection and Measurement, pp. 534 :John Wiley \& Sons, (2010).

${ }^{42}$ D. H. Beddingfield, N. H. Johnson, H. O. Menlove, "“3e neutron proportional counter performance in high gamma-ray dose environments," NIM A, Vol. 455, No. 3, pp 670-682, (2000).

${ }^{43}$ E. Trujillo, "National Instrument List Mode Data Acquisition (NILA) System: User's Manual," LA-UR-13-26782, August (2013).

${ }^{44} \mathrm{~J}$. Bagi et al, "Neutron coincidence counting with digital signal processing," Nuclear Instruments and Methods A, Vol. 608, No. 2, pp 316-327, (2009).

${ }^{45}$ M. T. Swinhoe, "Multiplicity Pulse Train Analysis for the ESARDA NDA Working Group Benchmark Exercise," LA-UR-04-7042, (2004).

${ }^{46}$ K. E. Koehler, V. Henzl, D. Henzlova, and P. Santi, "Characterizations of Double Pulsing in Neutron Multiplicity and Coincidence Counting Systems," To be submitted for publication, (2016).

${ }^{47}$ S. Croft, A. Favalli, D. K. Hauck, D. Henzlova, and P. A. Santi, "Feynman variance-to-mean in the context of passive neutron coincidence counting," NIM A, Vol. 686, pp 136-144, (2012).

${ }^{48}$ W. Hage, and D. M. Cifarelli, "Correlation Analysis with Neutron Count Distributions in Randomly or Signal Triggered Time Intervals for Assay of Special Fissile Materials," Nuclear Science and Engineering, Vol. 89, pp 159-176, (1985).

${ }^{49}$ J. R. Lamarsh, A. J. Baratta, Introduction to Nuclear Engineering, (Third edition) Prentice Hall (2001).

${ }^{50}$ K. J. Moody, I. D. Hutcheon, and P. M. Grant, Nuclear Forensic Analysis, CRC Press, (2014).

${ }^{51}$ J. R. Richard, M. L. Fensin, S. J. Tobin, M. T. Swinhoe, J. Baciak, and H. O. Menlove, "Characterization of the Neutron Source Term and Multiplicity of a Spent Fuel Assembly in Support 
of NDA Safeguards of Spent Nuclear Fuel," Institute of Nuclear Materials Management 51 st Annual Meeting, Baltimore, MD, July 11-16, (2010).

${ }^{52}$ H. R. Trellue, M. L. Fensin, J. R. Richard, J. Galloway and J. L. Conlin, "Description of the Spent Nuclear Fuel Used in the Next Generation Safeguards Initiative to Determine Plutonium Mass in Spent Fuel," Los Alamos National Laboratory, Los Alamos, (2010).

${ }^{53}$ J. D. Galloway, H. R. Trellue, M. L. Fensin, and B. L. Broadhead, "Design and Description of the NGSI Spent Fuel Library with an Emphasis on Passive Gamma Signal," Journal of Nuclear Materials Management, Vol. 40, No.3, pp 25 (2012).

${ }^{54}$ M. L. Fensin, S.J. Tobin et al., “A Monte Carlo linked Depletion Spent Fuel Library for Assessing Varied Nondestructive Assay Techniques for Nuclear Safeguards," Advances in Nuclear Fuel Management IV (ANFM), Hilton Head Island, SC USA, (2009).

${ }^{55}$ T. Goorley, M. James, T. Booth, F. Brown, J. Bull, L. J. Cox, J. Durkee, J. Elson, M. Fensin, R. A. Forster, J. Hendricks, H. G. Hughes, R. Johns, B. Kiedrowski, R. Martz, S. Mashnik, G. McKinney, D. Pelowitz, R. Prael, J. Sweezy, L.Waters, T.Wilcox, and T. Zukaitis, "Initial MCNP6 release overview," Nuclear Technology, Vol. 180, no. 3, pp 298-315, [Online]. Available: http://www.ans.org/pubs/journals/nt/a, 15346, December (2012).

${ }^{56}$ H. R. Trellue, "Development of Monteburns: a Code that Links MCNP and ORIGEN2 in an Automated Fashion for Burnup Calculations," LA-13514-T, Los Alamos National Laboratory (1998).

${ }^{57}$ W. B. Wilson et. al., “A Manual for CINDER'90 Version 07.4 Codes and Data,” Los Alamos National Laboratory, LA-UR-07-8412, (2008).

${ }^{58}$ D. Pelowitz (ed.), “MCNPX User's Manual”, Version 2.7.0, Los Alamos National Laboratory, LACP-11-00438, (2011).

59 A. Enqvist, M. Flaska, S. A. Pozzi, "Measurement and Simulation of Neutron/Gamma-Ray Cross-Correlation Functions from Spontaneous Fission”, Nuclear Instruments and Methods in Physics Research A, Vol. 595, pp 426-430, (2008).

${ }^{60}$ N. E. Holden, M. S. Zucker, “Average Prompt Emission Multiplicity (Nubar) Values at 2200 Meters Per Second for the Fissile Nuclides," Brookhaven National Laboratory, (1986).

${ }^{61}$ L. G. Evans, M. A. Schear, M. T. Swinhoe, S. J. Tobin, J. H. Hendricks, "New Capability for the PTRAC Capture File for Coincidence Counting (PTRAC): Induced Fission Isotope Identification by ZAID (first and last fission)", Unpublished internal guide, June (2010).

${ }^{62}$ T. Gozani, Active nondestructive assay of nuclear materials: principles and applications, US Nuclear Regulatory Commission, Washington, DC, (1981).

${ }^{63}$ H. O. Menlove, D. H. Beddingfield, "The Development of ${ }^{3}$ He Neutron Detectors for Applications in High Level Gamma-Ray Backgrounds," NDA and Nondestructive Examination Waste Characterization Conference, Salt Lake City, UT. October 24-26, (1995). 
${ }^{64}$ R. T. Kouzes, J. R. Ely, A. T. Lintereur, D. L. Stephens, "Neutron Detector Gamma Insensitivity Criteria," PNNL Report for the Department of Energy, (2009).

${ }^{65}$ E. Padovani, S. A. Pozzi, S. D. Clarke, E. C. Miller, "Introduction to MCNPX-PoliMi", Version 2.7, (2012).

${ }^{66}$ H. Tagziria, P. Peerani, "Monte Carlo Modelling of Neutron Coincidence Counting Systems for Nuclear Safeguards," International Conference on Mathematics, Computational Methods, and Reactor Physics, Saratoga Springs NY, May 3-7, (2009).

${ }^{67}$ N. Ensslin et al., "Application Guide to Neutron Multiplicity Counting," Los Alamos National Laboratory Manual, LA-13422-M, (1998).

${ }^{68}$ N. Ensslin, "Simple self-multiplication correction for in-plant use ," IAEA Symposium, Liege, Belgium, May 21, (1985).

${ }^{69} \mathrm{~K}$. Bohnel, "The effect of Multiplication on the Quantitative Determination of Spontaneously Fissioning Isotopes by Neutron Correlation Analysis," Nuclear Science and Engineering, Vol. 90, No. 1, pp 75-82, (1985).

${ }^{70} \mathrm{~K}$. Bohnel, "Determination of Plutonium in Nuclear Fuels Using the Neutron Coincidence Method," Kernforschungszentrum, Karlsruhe, KFK 2203 (1975).

${ }^{71}$ T. Nagatani et. al, "Experimental Studies of Passive Neutron Measurement for Fuel Debris at Fukushima Daiichi Nuclear Power Plants," Proceedings of the INMM $56^{\text {th }}$ Annual Meeting, Indian Wells, CA, July 12-16, (2015).

${ }^{72}$ M. A. Schear, H. O. Menlove, L. G. Evans, S. J. Tobin, S. Croft, "Spent fuel characterization using the differential die-away self-interrogation technique", Proceedings of the 52rd Annual Meeting of the Institute of Nuclear Materials Management, Palm Desert, CA July 17-21, (2011).

${ }^{73}$ S. J. Tobin, "Determining Plutonium Mass in Spent Fuel with Nondestructive Assay Techniques Preliminary Modeling Results Emphasizing Integration among Techniques," Global 2009, Paris, France, 6-11 September (2009).

${ }^{74}$ N. Miura, H. O. Menlove, "The Use of Curium Neutron to Verify Plutonium in Spent Fuel and Reprocessing Wastes," LANL report, LA-12774-MS, (1994).

${ }^{75}$ International Atomic Energy Agency, "Model Integrated Safeguards Approach for a Spent Fuel Encapsulation Plant," (2010).

${ }^{76}$ M. A. Schear, H. O. Menlove, L. G. Evans, S. J. Tobin, and S. Croft, "Development of the Differential Die-Away Self-Interrogation Technique for Spent Fuel Characterization," US Department of Energy report, (2010).

${ }^{77}$ A. M. Lafleur, W. S. Charlton, H. O. Menlove, M. T. Swinhoe, and A. R. Lebrun, "Development of Self-Interrogation Neutron Resonance Densitometry to Improve Detection of Possible Diversions for PWR Spent Fuel Assemblies" in American Nuclear Society Proceedings, Vol. 181, pp 354-370 (2013). 
${ }^{78}$ V. Henzl, "Evaluation of Differential Die-Away Technique Potential in Context of NonDestructive Assay of Spent Nuclear Fuel," Los Alamos National Laboratory report, LA-UR-1429224 (2014).

${ }^{79}$ A. C. Trahan, V. Henzl, A. P. Belian, M. T. Swinhoe, H. O. Menlove, M. Flaska, and S. A. Pozzi, "Utilizing Relative Total Pu Content in Spent Nuclear Fuel as a Tool for Identifying Fuel Pin Diversions," O.U.O., LA-CP-15-20449, (2015).

${ }^{80}$ A. C. Kaplan, V. Henzl, H. O. Menlove, M. T. Swinhoe, A. P. Belian, M. Flaska, S. A. Pozzi, "Partial Defect Scenario Detection with the Differential Die-Away Self-Interrogation Instrument," American Nuclear Society Student Conference, State College, PA Apr. 3-5, (2014).

${ }^{81}$ H. R. Trellue and D. I. Poston, "Preliminary Benchmarking of Monteburns, a Monte Carlo Burnup Code," American Nuclear Society 1999 Annual Meeting transactions, Boston, Massachusetts, Vol. 80, June 6-10, (1999).

${ }^{82}$ A. Lebrun, "NDA of LEU spent fuel”, American Nuclear Society, Vol. 135, No. 3, (2001).

${ }^{83} \mathrm{~W}$. Charlton et al., "Operator declaration verification technique for spent fuel at reprocessing facilities," NIM-B, Vol. 168, No. 1, pp 98-108, (2000).

${ }^{84} \mathrm{~A}$. M. Bolind, "The use of the BIC set in the characterization of used nuclear fuel assemblies by nondestructive assay," Annals of Nuclear Energy, Vol. 66, pp 31-50, (2014).

${ }^{85}$ H. O. Menlove, T. D. Reilly, "The verification of reactor operating history using the fork detector," $37^{\text {th }}$ Annual Meeting of the Institute of Nuclear Materials Management, Naples, FL July 28-August 1, (1996).

${ }^{86}$ S. Croft, R. D. McElroy, S. Philips, L. G. Evans, and M. F. Villani, "Deadtime Behaviors in Passive Neutron Coincidence Correlation Counting," Waste Management Symposia, WM Vol. 7 (2007).

${ }^{87}$ A. C. Kaplan, A. P. Belian, K. Koehler, V. Henzl, M. T. Swinhoe, H. O. Menlove, M. Flaska, S. A. Pozzi, "Characterization Measurements of the Differential Die-Away Self-Interrogation Instrument," Institute of Nuclear Materials Management Annual Meeting, Indian Wells, CA, July 11-17, (2015).

${ }^{88}$ J. E. Steward et al., "The Epithermal Neutron Multiplicity Counter Design and Performance Manual: More Rapid Plutonium and Uranium Inventory Verifcations by Factors of 5-20," Manual, LA-13743-M, (2000).

${ }^{89}$ G. Van Rossum, F. Drake (eds.) “Python Reference Manual,” Release 2.2.1, python.org (2002).

${ }^{90}$ M. S. Krick et al. "The IAEA Neutron Coincidence Counting (INCC) and the DEMING LeastSquares Fitting Programs.” United States: N. p., web, (1998).

${ }^{91}$ Unpublished data from Alison Goodsell, (2015). 
92 A. C. Trahan, A. P. Belian, M. T. Swinhoe, H. O. Menlove, M. Flaska, S. A. Pozzi, "Fresh Fuel Measurements with the Differential Die-Away Self-Interrogation Instrument," To be published, (2016).

${ }^{93}$ Ernst et al, "NEN-1 Fuel Rod Analysis Report,” LANL internal report, LA-UR-13-23581 (2013).

${ }^{94}$ M. A. Root, "Technical Basis for Use of Correlated Neutron Source in the Uranium Neutron Coincidence Collar," Thesis, Massachusetts Institute of Technology, September (2015).

95 S. R. Bolding, "Design of a Neutron Spectrometer and Simulations of Neutron Multiplicity Experiments with Nuclear Data Perturbations," Thesis, Kansas State University, (2011).

96 J. Mattingly et al., "An Evaluation of Monte Carlo Simulations of Neutron Multiplicity Measurements of Plutonium Metal,” ANS Annual Meeting, Chicago, IL, June 24-28, (2012).

${ }^{97}$ M. Monterial, M. Paff, S. Clarke, E. Miller, S. A. Pozzi, P. Marleau, S. Kiff, J. K. Mattingly, "Time-correlated-pulse-height technique measurements of fissile samples at the device assembly facility," Proceedings of the $54^{\text {th }}$ meeting of the Institute of Nuclear Materials Management, Palm Desert, CA (2013).

${ }^{98}$ M. Monterial, P. Marleau, M. Paff, S. Clarke and S. A. Pozzi, "Fission Chain Timing Distributions from Time-Correlated Gamma-Neutron Measurements of Subcritical Plutonium Assemblies," to be published, (2016).

${ }^{99}$ S. J. Tobin, H. O. Menlove, M. T. Swinhoe, M. A. Schear, "Next Generation Safeguards Initiative research to determine the $\mathrm{Pu}$ mass in spent fuel assemblies: Purpose, approach, constraints, implementation, and calibration," Nuclear Instruments and Methods in Physics Research Section A, Vol. 652, No. 1, pp 73-75, 1 October (2011).

${ }^{100}$ V. V. Mozin, S. J. Tobin, L. W. Cambell, J. R. Cheatham, C. R. Freeman, C. J. Gesh, "Determining Plutonium Mass in Spent Fuel with Nondestructive Assay Techniques NGSI Research Overview and Update on NDA Techniques." Lawrence Berkeley National Laboratory: Lawrence Berkeley National Laboratory. LBNL Paper LBNL-5174E. Retrieved from: http://escholarship.org/uc/item/8df350x5, (2012).

101 M. J. Joyce, M. D. Aspinall, F. D. Cave, A. D. Lavietes, "Real-time, digital pulse-shape discrimination in non-hazardous fast liquid scintillation detectors: Prospects for safety and security," IEEE Transactions on Nuclear Science, Vol. 59, No. 4, pp 1245-1251, August (2012).

102 L. Stevanato, D. Cester, G. Nebbia, G. Viesti, "Neutron detection in a high gamma-ray background with EJ-301 and EJ-309 liquid scintillators," Nuclear Instruments and Methods in Physics Research Section A: Accelerators, Spectrometers, Detectors and Associated Equipment, Vol. 690, No. 1, pp 96-101, 4 July (2012).

103 A. Dougen, et al., "New and Novel Nondestructive Neutron and Gamma-Ray Technologies," JAEA-IAEA Workshop on Advanced Safeguards Technology for the Future Nuclear Fuel Cycle, Takai-Mura, Japan, November 12 - 17, (2007). 
${ }^{104}$ M. Flaska, S. A. Pozzi, "Identification of shielded neutron sources with the liquid scintillator BC501A using a digital pulse shape discrimination method", Nuclear Instruments and Methods in Physics Research Section A: Accelerators, Spectrometers, Detectors and Associated Equipment, Vol. 577, No. 3, pp 654-663, 11 July (2007).

${ }^{105}$ S. A. Pozzi, S. D. Clarke, M. Flaska, and P. Peerani, "Pulse-height distributions of neutron and gamma rays from plutonium-oxide samples," Nuclear Instruments and Methods in Physics Research Section A: Accelerators, Spectrometers, Detectors and Associated Equipment, Vol. 608, No. 2, pp 310-315, September (2009).

${ }^{106}$ I. A. Pawełczak, S. A. Ouedraogo, A. M. Glenn, R. E. Wurtz, L. F. Nakae, "Studies of neutron pulse shape discrimination in EJ-309 liquid scintillator using charge integration method," Nuclear Instruments and Methods in Physics Research Section A, Vol. 711, pp 21-26, 24 January (2013). 